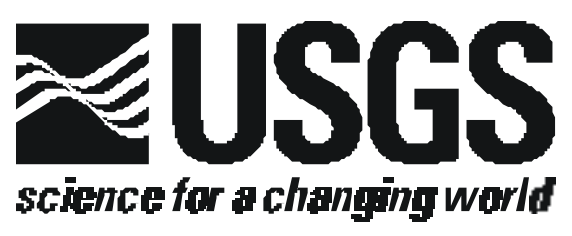

\title{
MAGNETOTELLURIC DATA ACROSS THE BATTLE MOUNTAIN-EUREKA AND CARLIN TRENDS, NORTH OF EUREKA, NEVADA
}

\author{
by \\ Jackie M. Williams \\ and \\ Brian D. Rodriguez 1
}

Open-File Report 01-168

2001

This report is preliminary and has not been reviewed for conformity with U.S. Geological Survey editorial standards and stratigraphic nomenclature. Any use of trade, product, or firm names is for descriptive purposes only and does not imply endorsement by the U.S.

Government.

U.S. DEPARTMENT OF THE INTERIOR

U.S. GEOLOGICAL SURVEY

${ }^{1}$ Denver, Colorado 
TABLE OF CONTENTS

INTRODUCTION

MAGNETOTELLURIC METHOD 3

MAGNETOTELLURIC SURVEY

MAGNETOTELLURIC DATA

REFERENCES CITED $\quad 9$

APPENDIX - MAGNETOTELLURIC DATA PLOTS 11 


\section{INTRODUCTION}

Genesis of gold deposits along the Battle Mountain-Eureka and Carlin trends in northern Nevada is not fully understood and subject to conflicting models (e.g. Arehart and others, 1993; Ilchik and Barton, 1997; Radtke, 1985; Shawe, 1991; Sillitoe and Bonham, 1990; Tosdal, 1998). A general consensus among these models is that regional structures somehow controlled the spatial distribution of the deposits. To investigate crustal structures that may be related to the genesis of gold deposits along these trends, a regional southwest-northeast profile of magnetotelluric (MT) soundings was acquired in 1999 and 2000 (line MT1-MT1", Figure 1). Resistivity modeling of the MT data can be used to infer the deep resistivity structure of the crust to help investigate possible tectonic controls on the emplacement of mineral deposits along these linear trends. The purpose of this report is to release the MT sounding data.

\section{MAGNETOTELLURIC METHOD}

The magnetotelluric (MT) method is a passive surface geophysical technique, which uses the earth's natural electromagnetic fields to investigate the electrical resistivity structure of the subsurface. The resistivity of geologic units is largely dependent upon their fluid content, porosity, degree of fracturing, temperature, and conductive mineral content (Keller, 1989). Saline fluids within the pore spaces and fracture openings can reduce resistivities in a resistive rock matrix. Also, resistivity can be lowered by the presence of conductive clay minerals, carbon, and metallic mineralization. It is common for altered volcanic rocks to contain authigenic minerals that have resistivities ten times lower than those of the surrounding rocks (Nelson and Anderson, 1992). Increased temperatures cause higher ionic mobility and mineral activation energy, reducing rock resistivities significantly. Unaltered, unfractured igneous rocks are normally very resistive (typically 1,000 ohm-m or greater), whereas fault zones will show low resistivity (less than $100 \mathrm{ohm}-\mathrm{m}$ ) when they are comprised of rocks fractured enough to have hosted fluid transport and consequent mineralogical alteration (Eberhart-Phillips and others, 1995). Carbonate rocks are moderately to highly resistive (hundreds to thousands of ohm-m) dependent upon their fluid content, porosity, fracturing, and impurities. Marine shales, mudstones, and clay-rich alluvium are normally very conductive (a few ohm-m to tens of ohm-m). Unaltered, metamorphic rocks (non-graphitic) are moderately to highly resistive (hundreds to thousands of ohm-m). Tables of electrical resistivity for 
a variety of rocks, minerals and geological environments may be found in Keller (1987) and Palacky (1987).

The MT method can be used to probe the crust from depths of tens of meters to depths of tens of kilometers (Vozoff, 1991). Natural variations of the Earth's magnetic and electric field are measured and recorded at each MT station. The main frequency bands used by the MT method are $10,000 \mathrm{~Hz}$ to $1 \mathrm{~Hz}$ from worldwide lightning activity and $1 \mathrm{~Hz}$ to 0.0001 $\mathrm{Hz}$ from geomagnetic micro-pulsations. The natural electric and magnetic fields propagate vertically in the earth because the very large resistivity contrast between the air and the earth causes a vertical refraction of both fields transmitted into the earth (Vozoff, 1972).

The natural electric and magnetic fields are recorded in two orthogonal, horizontal directions. The vertical magnetic field ("tipper") is also recorded. The resulting time-series signals are used to derive earth tensor apparent resistivities and phases by first converting them to complex cross-spectra using FFT (fast-Fourier-transform) techniques. Least-squares, cross-spectral analysis (Bendat and Piersol, 1971) is used to solve for a tensor-transfer function that relates the observed electric fields to the magnetic fields under the assumption that the Earth consists of a two-input, two-output, linear system with the magnetic fields as input and the electric fields as output (Rodriguez and others, 1996). Prior to conversion to apparent resistivity and phase, the tensor is normally rotated into principal directions that correspond to the direction of maximum and minimum apparent resistivity. For a two-dimensional (2-D) Earth, the MT fields can be decoupled into transverse electric (TE) and transverse magnetic (TM) modes; 2-D modeling is generally done to fit both modes. When the geology satisfies the 2-D assumption, the MT data for the TE mode is assumed to represent the situation when the electric field is along the geologic strike, and the data for the TM mode is assumed to represent the situation when the electric field is across strike. The MT method is well suited for studying complicated geological environments because the electric and magnetic relations are sensitive to vertical and horizontal variations in resistivity. The method is capable of establishing whether the electromagnetic fields are responding to subsurface terranes of effectively 1-, 2-, or 3dimensions. An introduction to the MT method and references for a more advanced understanding are contained in Dobrin and Savit (1988) and Vozoff (1991). 


\section{MAGNETOTELLURIC SURVEY}

Fourteen MT soundings were located along or near profile MT1-MT1" (Figure 1) with spacing that varied from 1.3 to 16.9 kilometers. The profile orientation is roughly perpendicular to the Battle Mountain-Eureka and Carlin trends. All stations were collected with our portable EMI MT-1 system (EMI, 1996). The horizontal electric fields were sensed using an L-shaped, three-electrode array with dipole lengths of $30 \mathrm{~m}$. The orthogonal, horizontal magnetic fields in the direction of the electric-field measurement array were sensed using permalloycored induction coils (Stanley and Tinkler, 1983). Frequencies sampled ranged from 100 to $0.01 \mathrm{~Hz}$ using single station recordings of both orthogonal horizontal components of the electric and magnetic fields, along with the vertical magnetic field at all stations except station 78. Sampling this frequency range in previous areas of widely varying geology has allowed us to probe the crust from depths of hundreds of meters to depths of tens of kilometers.

The recorded time-series data were transformed to the frequency domain and Fourier analyzed to determine a twodimensional apparent resistivity and phase tensor at each site. The data were rotated to maximum and minimum apparent resistivity directions so that propagation modes for the signals were decoupled into TE and TM modes. Reference sensors to help reduce bias in the impedance determinations due to instrument or environmental noise (Gamble and others, 1979a; Clarke and others, 1983) were not used. Although remote reference techniques were not used in our survey, we did sort cross-power files to select optimal signal-to-noise data sets (Appendix).

The effects of near-surface resistivity anomalies cause "static shifts" (Sternberg and others, 1988) in the data. Static shifts of this data set ranged from 0.0 to 1.3 of a log decade. Only station 80 had a static shift of 1.3 of a log decade. The remainder of the stations had an average of 0.2 of a log decade static shift.

\section{MAGNETOTELIURIC DATA}

The following table shows fourteen magnetotelluric (MT) station locations (from southwest to northeast). Coordinates are referenced to the 1866 Clarke spheroid and North American 1927 Western United States datum. Longitude and latitude format below is decimal degrees. Elevation is in meters. 


\begin{tabular}{|c|c|c|c|}
\hline Station & Longitude & Latitude & Elev (m) \\
\hline 88 & -116.47773 & $\overline{39.52744}$ & 1890 \\
\hline 87 & -116.27814 & 39.55249 & 1850 \\
\hline 86 & -116.15948 & 39.60576 & 1870 \\
\hline 85 & -116.04913 & 39.62802 & 1820 \\
\hline 89 & -115.97073 & 39.75141 & 1780 \\
\hline 109 & -115.87795 & 39.70953 & 1870 \\
\hline 83 & -115.80588 & 39.73506 & 2050 \\
\hline 82 & -115.71711 & 39.79929 & 1780 \\
\hline 81 & -115.63851 & 39.79379 & 1810 \\
\hline 80 & -115.54086 & 39.77805 & 2170 \\
\hline 108 & -115.53142 & 39.76552 & 2120 \\
\hline 79 & -115.47101 & 39.80462 & 2020 \\
\hline 77 & -115.39775 & 39.81638 & 1870 \\
\hline 78 & -115.32175 & 39.83858 & 1910 \\
\hline
\end{tabular}

The figures in the Appendix represent the field-processed MT data for each station after the time series data were converted to the frequency domain and the tensor-transfer function was rotated into principal directions as described above in the "Magnetotelluric Method" section.

For each station, nine separate graph plots are given:

1. Apparent Resistivity for the rotated maximum ( $x$ symbol) and minimum (o symbol) modes

2. Impedance Phase for the rotated maximum ( $x$ symbol) and minimum (o symbol) modes

3. Rotation Angle for the impedance tensor (corresponds to the direction of maximum apparent resistivity)

4. Impedance skew for the impedance tensor

5. Multiple Coherency for the rotated maximum ( $x$ symbol) and minimum (o symbol) modes of the electric field

6. Impedance Polar Plots (at 12 selected frequencies)

7. Tipper Magnitude for the vertical magnetic field

8. Tipper strike for the vertical magnetic field, and

9. $\mathrm{HzHx}$ ( $x$ symbol) and HzHy (o symbol) Coherency

Error bars that appear on the Apparent Resistivity, Impedance Phase, Skew, Tipper Magnitude, and Tipper strike plots represent probable errors within one standard deviation of the sample variance (Gamble and others, 1979b).

Apparent resistivity is a measure of the magnitude of the electric field strength over the magnetic field strength for a given frequency. The impedance phase is proportional to the slope of the apparent resistivity curve on a log-log scale, but from a baseline at -45 degrees (Vozoff, 1991). A measure 
of the dimensionality for $\mathrm{MT}$ data is provided by the impedance skew of the impedance tensor (Vozoff, 1972). If the effective measured resistivity response to the geology beneath a MT station is truly $1-D$ or $2-D$, then the skew will be zero. Both instrument and environmental sources of noise contribute to non-zero skew values, but are typically small (about 0.1) for relatively low noise level recordings. Higher skews (above 0.2) are an indication of either 3-D resistivity responses to the geology or higher levels of noise. Man-made electrical noise, such as power lines, power generators, moving vehicles and trains can have a negative effect on $\mathrm{MT}$ data quality. All these local disturbances produce an incoherent noise mainly affecting frequencies above $1 \mathrm{~Hz}$. other man-made electrical noise, such as direct current electric trains and active cathodic protection of pipelines produce coherent electromagnetic signals mainly affecting frequencies below $1 \mathrm{~Hz}$.

In the survey area, noise from a number of small power lines and small moving vehicles was negligible at distances of $0.4 \mathrm{~km}$ and greater from the noise source. Power line levels were measured at each site and were typically less than 20\% of the maximum recordable signals. Noise from larger power lines, power generators, pipelines, railroads, and steamdriven trains, mostly near mining operations, was negligible at least $5 \mathrm{~km}$ from them. Recordings were not made when noise from moving vehicles affected the magnetic signals. Local lightning, wind, and rainstorms can also degrade data quality, but these were avoided by not recording during active thunderstorm periods. Wind noise was minimized by burying the magnetic induction coils.

Predicted values of the electric field can be computed from the measured values of the magnetic field (Vozoff, 1991). The coherence of the predicted electric field with the measured electric field is a measure of the signal-to-noise ratio provided in the multiple coherency plot. Values are normalized between 0 and 1 , where values at 0.5 signify signal levels equal to noise levels. For this data set, coherencies were generally at an acceptable level, except at times in the "dead band" (0.1 to $1 \mathrm{~Hz})$ and at times in the lower frequencies $(0.01$ to 0.1$)$. The lower frequency ionospheric signals are related to sunspot activity whose levels typically follow an 11-year cycle. The sunspot activity was near the highest level of the cycle during the 1999 and 2000 surveys.

The figures in the Appendix represent the raw field MT data at each station, which includes some data scatter and 
poor signal-to-noise ratios. Our only effort at removing noisy data points was to visually inspect and select the best signal-to-noise field data to combine into the final data plots.

The impedance polar plots provide a measure of the MT data dimensionality (Reddy and others, 1977). For 1-D resistivity structures, the principal impedance polar diagram (dashed line) is a circle. For 2-D or 3-D resistivity structures, the principal impedance polar diagram (dashed line) elongates either parallel or perpendicular to strike direction. Over resistors, the principal impedance polar diagram elongates perpendicular to strike direction and over conductors, the principal impedance polar diagram elongates parallel to strike direction. Also, for 2-D resistivity structures, the additional impedance polar diagram (solid line) attains the shape of a symmetric clover leaf. For 3-D resistivity structures, the additional impedance polar diagram (solid line) elongates in one direction and its amplitude is comparable to that of the principal impedance polar diagram (dashed line). Sites whose polar plots indicated 3-D character in the lower frequencies were MT stations 77, 80, $82,83,86,88$, and 89 (Figure 1).

The tipper can be solved for when the vertical component of the magnetic field is measured. The tipper magnitude is a measure of the tipping of the magnetic field out of the horizontal plane (Vozoff, 1991). The magnitude is zero for the $1-\mathrm{D}$ case and typically increases between 0.1 to 0.5 , and rarely as great as 1 , as it responds to vertical and subvertical structures. The tipper strike is typically used to help resolve the 90-degree ambiguity in the impedance rotation angle. The vertical component of the magnetic field was measured at all MT stations except station 78. The tipper magnitude of these stations was typically 0.1 to 0.4 over the lower frequencies indicating vertical structure at depth. The $\mathrm{HzHx}$ and HzHy coherency is a measure of the signal-to-noise ratio of the vertical magnetic field with respect to each of the orthogonal horizontal magnetic field directions. Values are normalized between 0 and 1 , where values at 0.5 signify signal levels equal to noise levels. These three-component magnetic field coherencies provide a check on the signal-tonoise ratio of the measured values in the tipper magnitude and tipper strike plots. 


\section{REFERENCES CITED}

Arehart, G.B., Foland, K.A., Naeser, C.W., and Kesler, S.E., 1993, 40Ar/39Ar, K/Ar, and fission track geochronology of sediment-hosted disseminated gold deposits at post-Betze, Carlin Trend, northeastern Nevada: Economic Geology, vol. 88 , no. 3, p. 622-646.

Bendat, J.S., and Piersol, A.G., 1971, Random Data: Analysis and Measurement Procedures: New York, Wiley Interscience, $407 \mathrm{p}$.

Clarke, J., Gamble, T.D., Goubau, W.M., Koch, R.H., and Miracky, R.F., 1983, Remote-reference magnetotellurics: Equipment and procedures: Geophysical Prospecting, vol. 31, p. 149-170.

Dobrin, M.D., and Savit, C.H., 1988, Introduction to Geophysical Prospecting (4th ed.): New York, McGraw-Hill, $867 \mathrm{p}$.

Eberhart-Phillips, D., Stanley, W. D., Rodriguez, B. D. and Lutter, W. J., 1995, Surface seismic and electrical methods to detect fluids related to faulting: Journal of Geophysical Research, vol. 100, no. B7, pp. 12,91912,936 .

EMI, 1996, MT-1 magnetotelluric system operation manual, version 3.2: ElectroMagnetic Instruments, Inc., Richmond, California, $220 \mathrm{p}$.

Gamble, T.D., Goubau, W.M. and Clarke, J., 1979a, Magnetotellurics with a remote magnetic reference: Geophysics, v. 44, no. 1, p. 53-68.

Gamble, T.D., Goubau, W.M. and Clarke, J., 1979b, Error analysis for remote reference magnetotellurics: Geophysics, v. 44, no. 5, p. 959-968.

Ilchik, R.P. and Barton, M.D., 1997, An amagmatic origin of Carlin-type gold deposits: Economic Geology, vol. 92, no. $3, p, 269-288$.

Keller, G.V., 1987, Rock and mineral properties, in Electromagnetic Methods in Applied Geophysics Theory: M.N. Nabighian, Ed., Society of Exploration Geophysicists, Tulsa, Oklahoma, v. 1, p. 1351. 
Keller, G.V., 1989, Electrical properties, in Carmichael, R.S., Ed., Practical handbook of physical properties of rocks and minerals: CRC Press, Boca Raton, Florida, p. 359-427.

Nelson, P.H. and Anderson, L.A., 1992, Physical properties of ash flow tuff from Yucca Mountain, Nevada: Journal of Geophysical Research, vol. 97, no. B5, p. 6823-6841.

Palacky, G.J., 1987, Resistivity characteristics of geologic targets, in Electromagnetic Methods in Applied Geophysics Theory: M.N. Nabighian, Ed., Society of Exploration Geophysicists, Tulsa, Oklahoma, vol. 1, p. 53129.

Radtke, A.S., 1985, Geology of the Carlin gold deposit, Nevada: U.S. Geological Survey Professional Paper 1267, $124 \mathrm{p}$.

Reddy, I.K., Rankin, D., and Phillips, R.J., 1977, Threedimensional modelling in magnetotelluric and magnetic variational sounding: Geophysics Journal of the Royal Astronomical Society, vol. 51, p. 313-325.

Rodriguez, B.D., Stanley, W.D., and Williams, J.M., 1996, Axial structures within the Reelfoot rift delineated with magnetotelluric surveys: U.S. Geological Survey Professional Paper 1538-K, $30 \mathrm{p}$.

Shawe, D.R., 1991, Structurally controlled gold trends imply large gold resources in Nevada, in Geology and ore deposits of the Great Basin, Symposium Proceedings: Raines, G.L., Lisle, R.E., Schafe, R.W., Wilkinson, W.H., Eds., Geological Society of Nevada, Reno, vol. 1, p. 199212 .

Sillitoe, R.H. and Bonham, H.F., 1990, Sediment-hosted gold deposits; distal products of magmatic-hydrothermal systems: Geology, vol. 18, no. 2, p. 157-161.

Stanley, W.D., and Tinkler, R.D., 1983, A practical, low-noise coil system for magnetotellurics: U.S. Geological Survey Open-File Report 83-85, $18 \mathrm{p}$.

Sternberg, B.K., Washburne, J.C., and Pellerin, L., 1988, Correction for the static shift in magnetotellurics using transient electromagnetic soundings: Geophysics, vol. 53, p. 1459-1468. 
Struhsacker, E.M., Jones, E., and Green, S.M., 1996, Roadside geology and precious-metal mineralization along the I-80 corridor, Reno to Elko, Nevada, in Struhsacker, E.M. and Green, S.M., eds., Geology and ore deposits of the American Cordillera - Field Trip Guidebook Compendium: Geological Society of Nevada, Reno, Nevada, p. 3.

Tosdal, R.M., 1998, Contributions to the gold metallogeny of northern Nevada: U.S. Geological Survey Open-File Report $98-338,290 \mathrm{p}$.

Vozoff, K., 1972, The magnetotelluric method in the Exploration of sedimentary basins: Geophysics, vol. 37, p. $98-141$.

Vozoff, K., 1991, The magnetotelluric method, in Electromagnetic methods in applied geophysics: M.N. Nabighian, Ed., Society of Exploration Geophysicists, Tulsa, Oklahoma, vol. 2, part B, p. 641-711.

\section{APPENDIX}

\section{MAGNETOTELLURIC DATA PLOTS}

For stations 77, 79-89, 108, and 109, there are nine separate graph plots:

1. Apparent Resistivity for the rotated maximum ( $x$ symbol) and minimum (o symbol) modes

2. Impedance Phase for the rotated maximum ( $x$ symbol) and minimum (o symbol) modes

3. Rotation Angle for the impedance tensor (corresponds to the direction of maximum apparent resistivity)

4. Impedance skew for the impedance tensor

5. Multiple Coherency for the rotated maximum ( $x$ symbol) and minimum (o symbol) modes of the electric field

6. Impedance Polar Plots (at 12 selected frequencies)

7. Tipper Magnitude for the vertical magnetic field

8. Tipper Strike for the vertical magnetic field, and

9. $\mathrm{HzHx}$ ( $x$ symbol) and $\mathrm{HzHy}$ (o symbol) Coherency

Station 78 has only the first six graph plots above, since the vertical magnetic field data (Tipper, Hz) was not acquired. Refer to the "Magnetotelluric Data" section in this report for an explanation of these plots. 


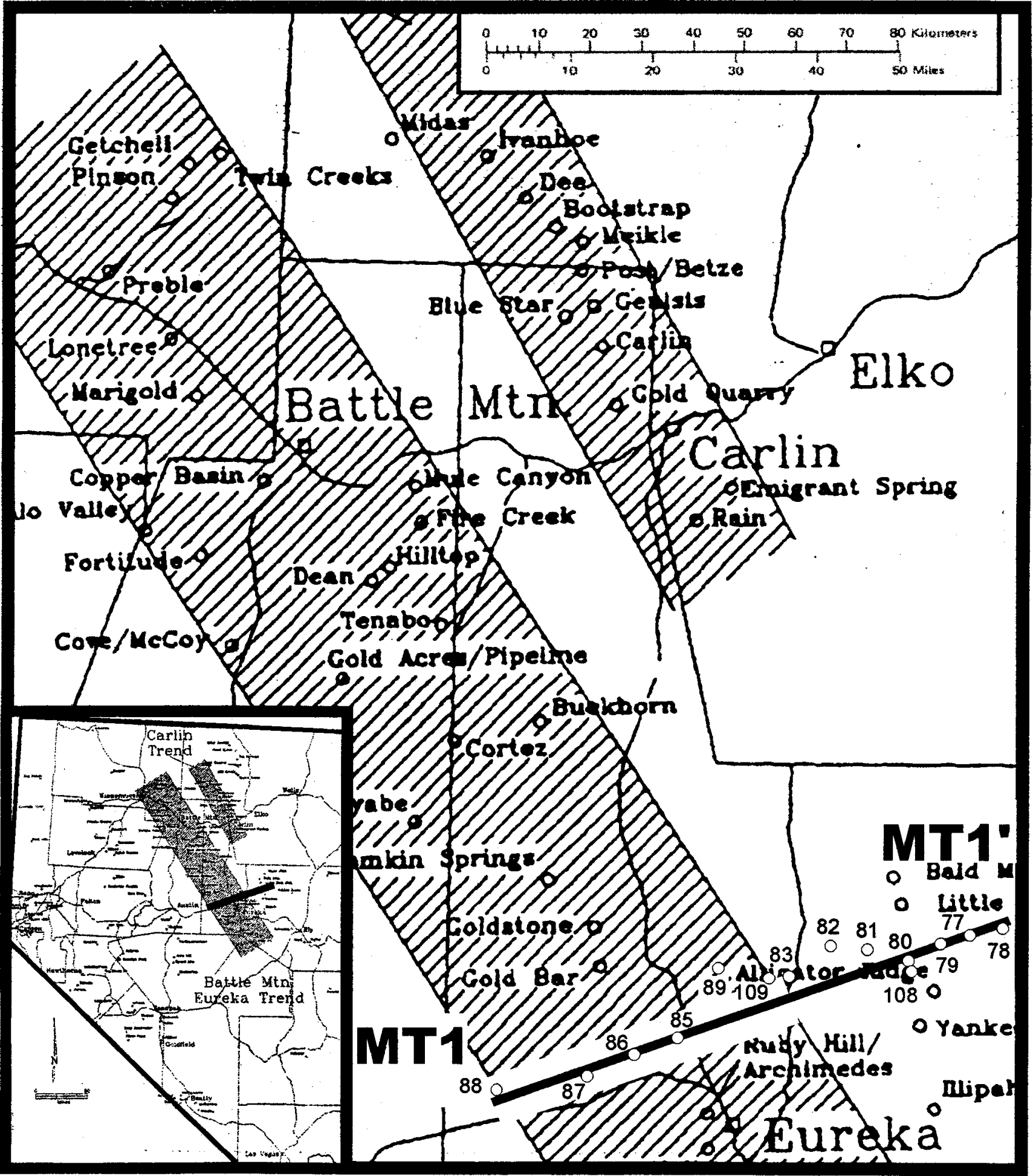

Figure 1. Index map. Magnetotelluric transect (MT1-MT1') acquired in 1999 and 2000 in northeastern Nevada. Shaded zones are two northwest-trending mineralized belts in northeastern Nevada, the well-known Carlin trend and the Battle Mountain-Eureka trend. Base map adapted from struhsacker and others $(1996)$. 


\section{APPARENT RESISTIVITY}

Kobeh Valley, NV

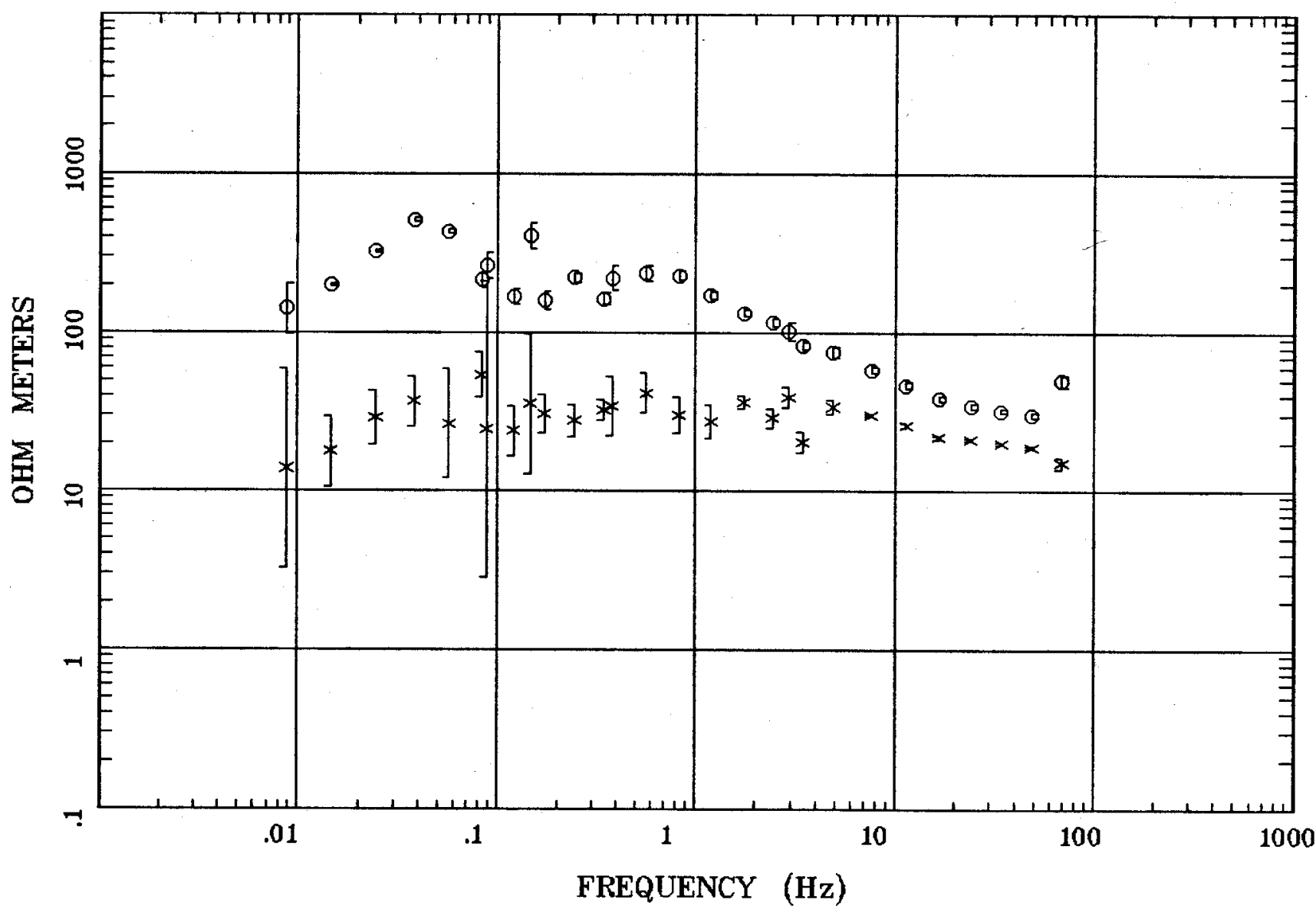

Client:

Remote: none

Acquired: 12:4 Aug 09, 1999

Survey Co:USGS
Rotation:

Filename: ar88b.avg

Channels: Ch1 Ch2 Ch3 Ch4 Ch5 Ch3 Ch4

Plotted: 11:07 Dec 08, 2000,

$<$ EMI - ElectroMagnetic Instruments 
IMPEDANCE PHASE

Kobeh Valley, NV

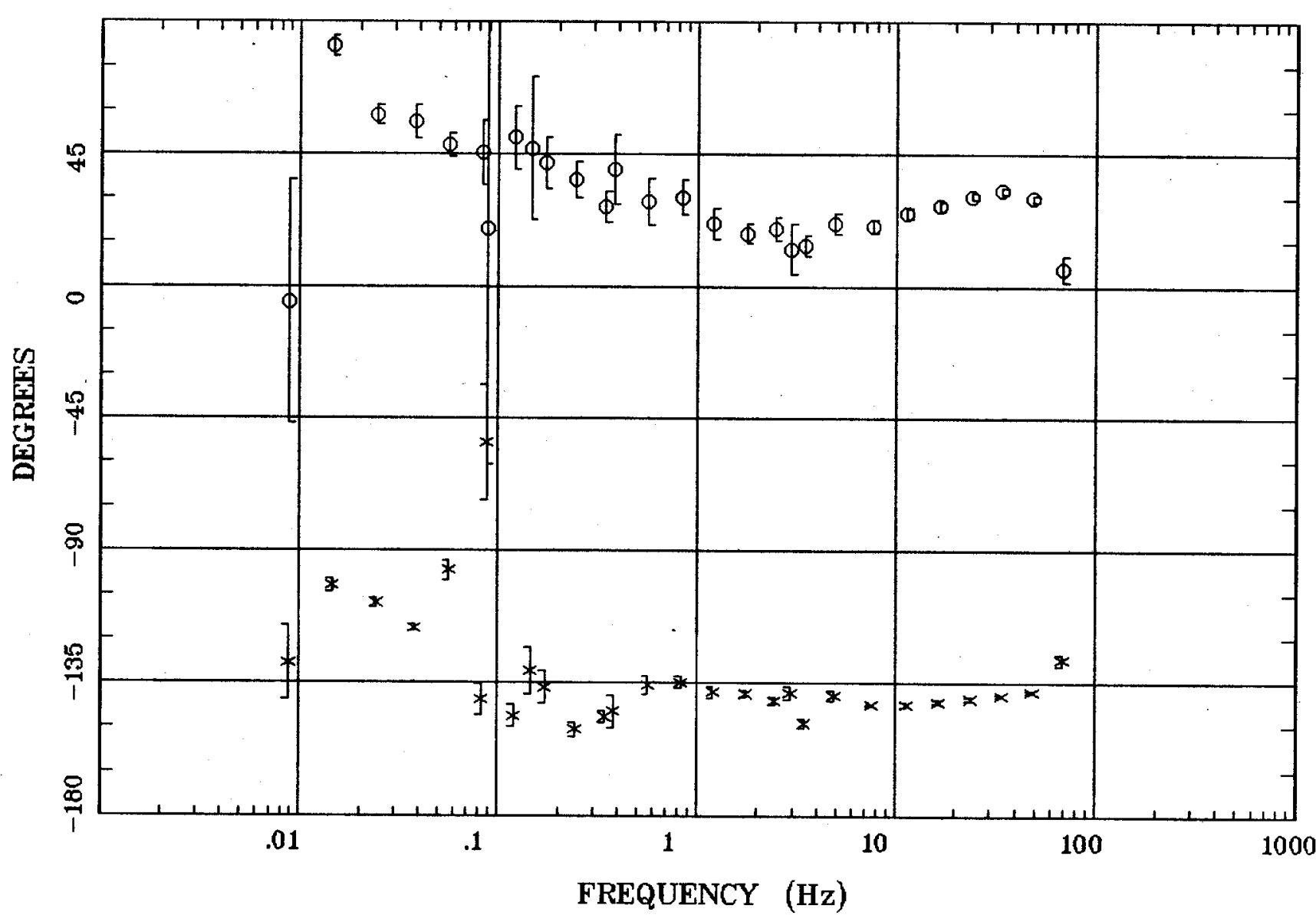

Client:

Remote: none

Acquired: 12:4 Aug 09, 1999

Survey Co:USGS

\section{Rotation:}

Filename: ar88b.avg

Channels: Ch1 Ch2 Ch3 ch4 Ch5 Ch3 Ch4 Plotted: 11:07 Dec 08, 2000

< EMI - ElectroMagnetic Instruments > 
ROTATION ANGLE

Kobeh Valley, NV

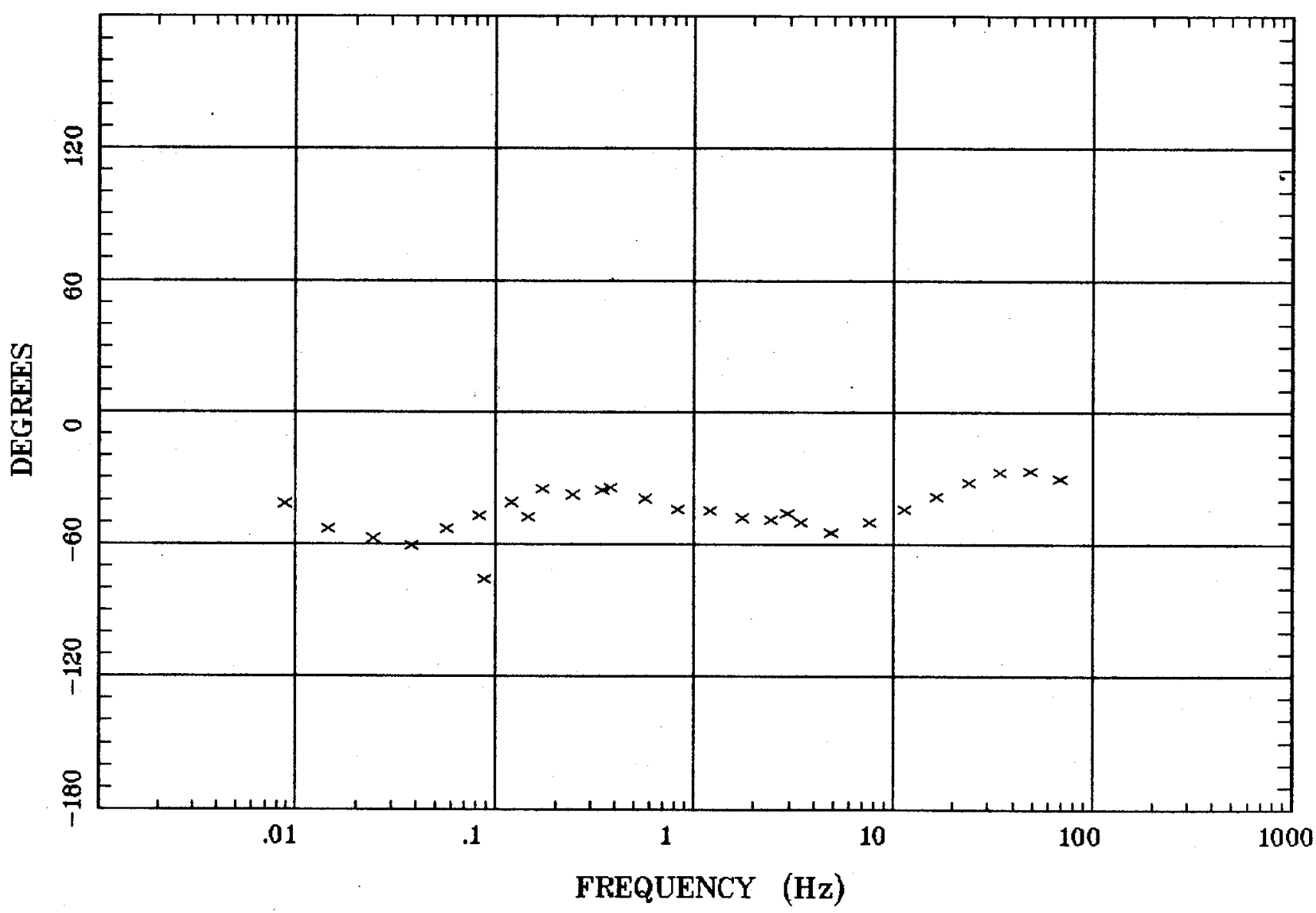

Client:

Remote: none

Acquired: 12:4 Aug 09, 1999

Survey Co:USGS
Rotation:

Filename: ar88b.avg

Channels: Ch1 Ch2 Ch3 Ch4 Ch5 Ch3 Ch4

Plotted: 11:07 Dec 08, 2000

< EMI - ElectroMagnetic Instruments > 


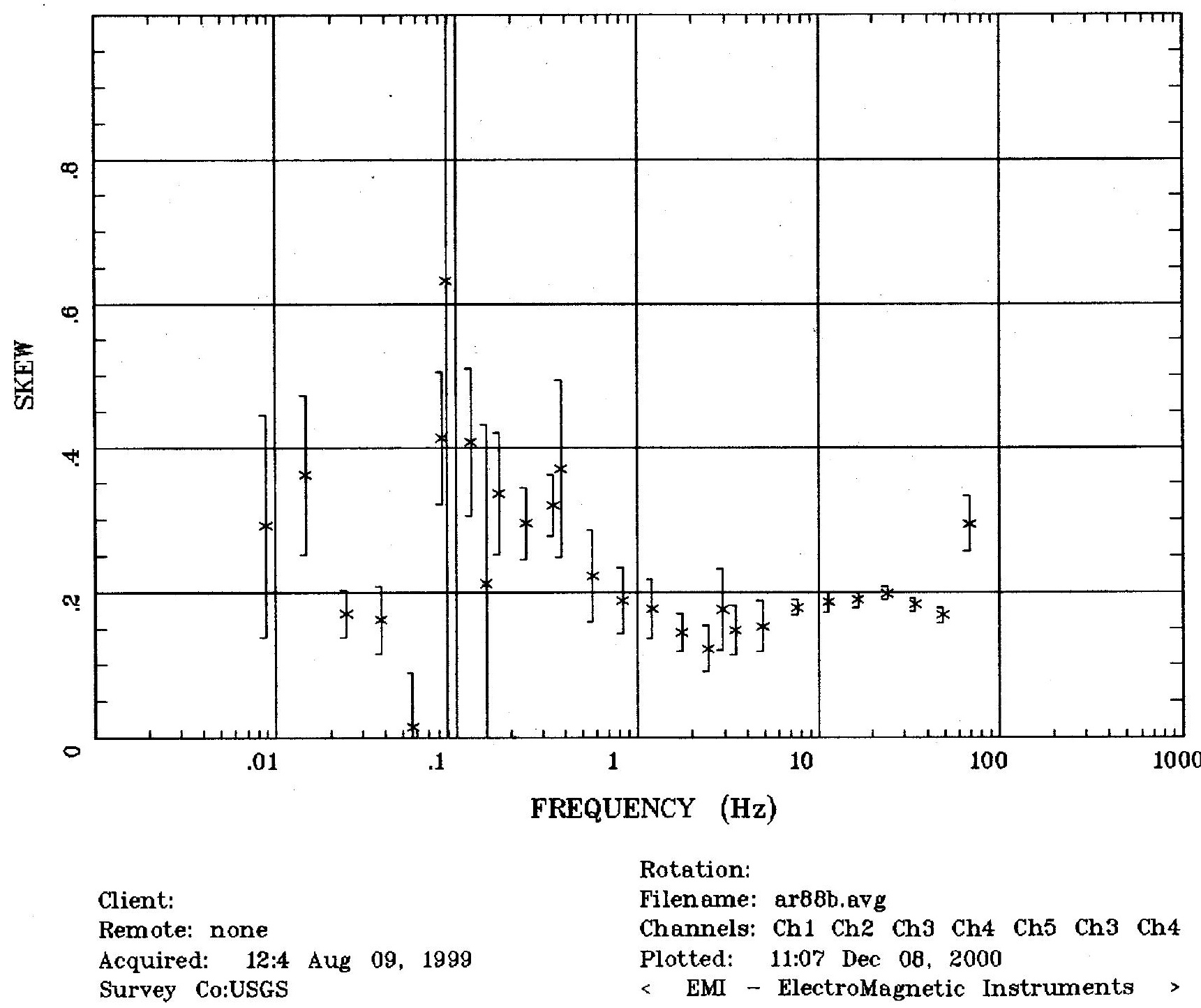


E MULT Coh. Kobeh Valley, NV

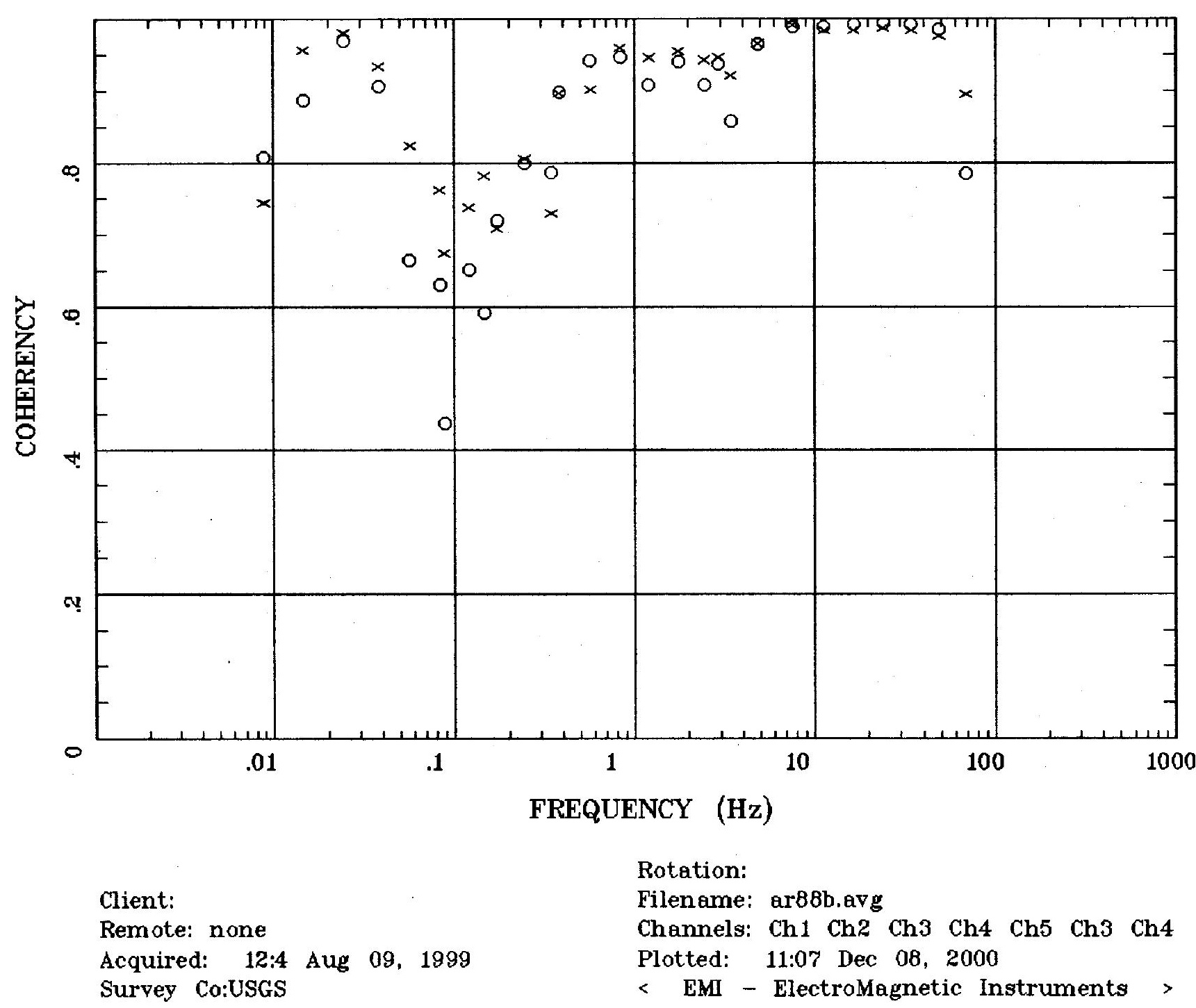


Station 88

POLAR PLOTS

Kobeh ,Valley, NV

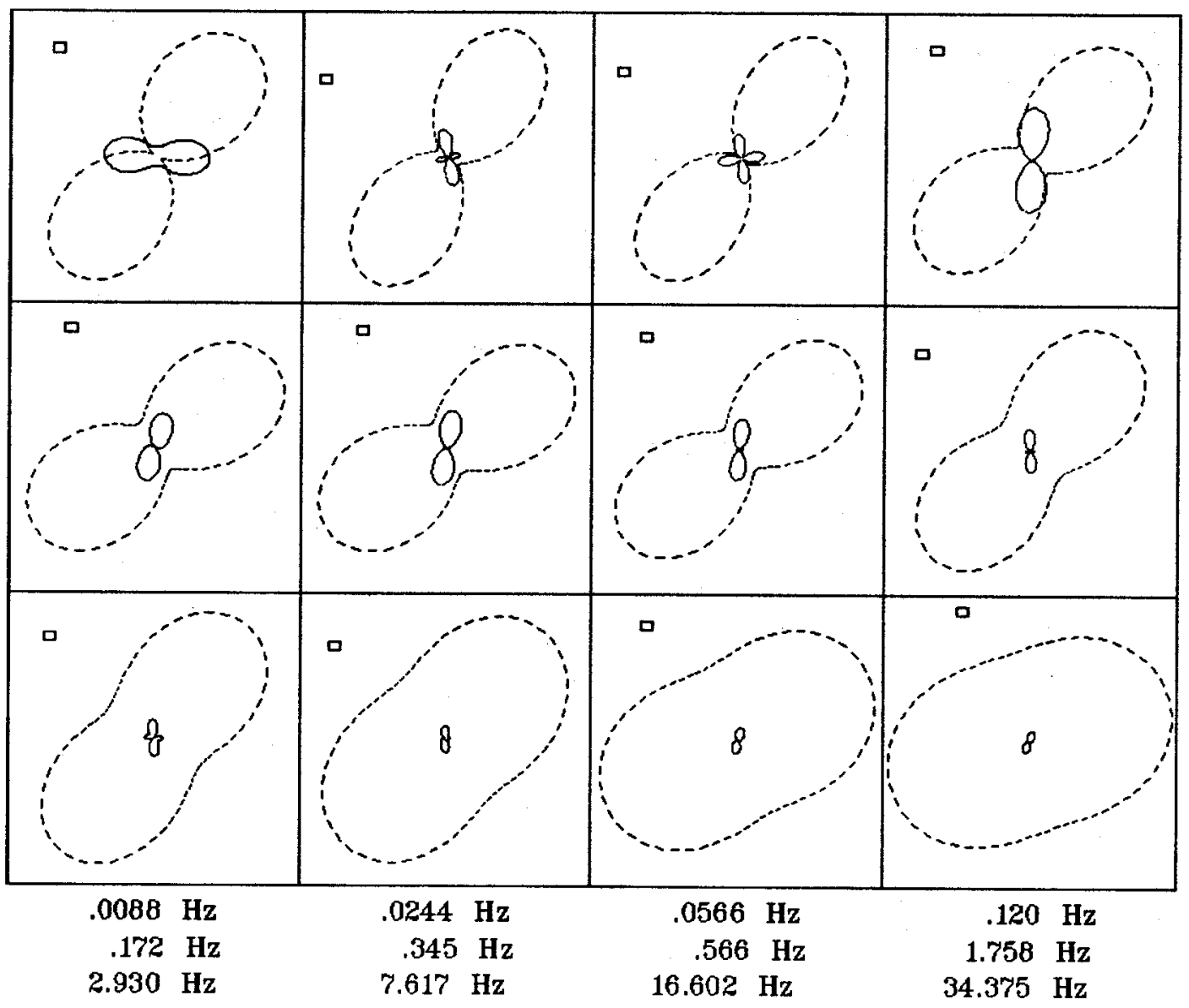

Client:

Remote: none

Acquired: 12:4 Aug 09, 1999

Survey Co:USGS

\section{Rotation:}

Filename: ar88b.avg

Channels: Ch1 Ch2 Ch3 Ch4 Ch5 Ch3 Ch4

Plotted: 11:07 Dec 08, 2000

< EMI - ElectroMagnetic Instruments > 
TIPPER MAGNITUDE

Kobeh Valley, NV

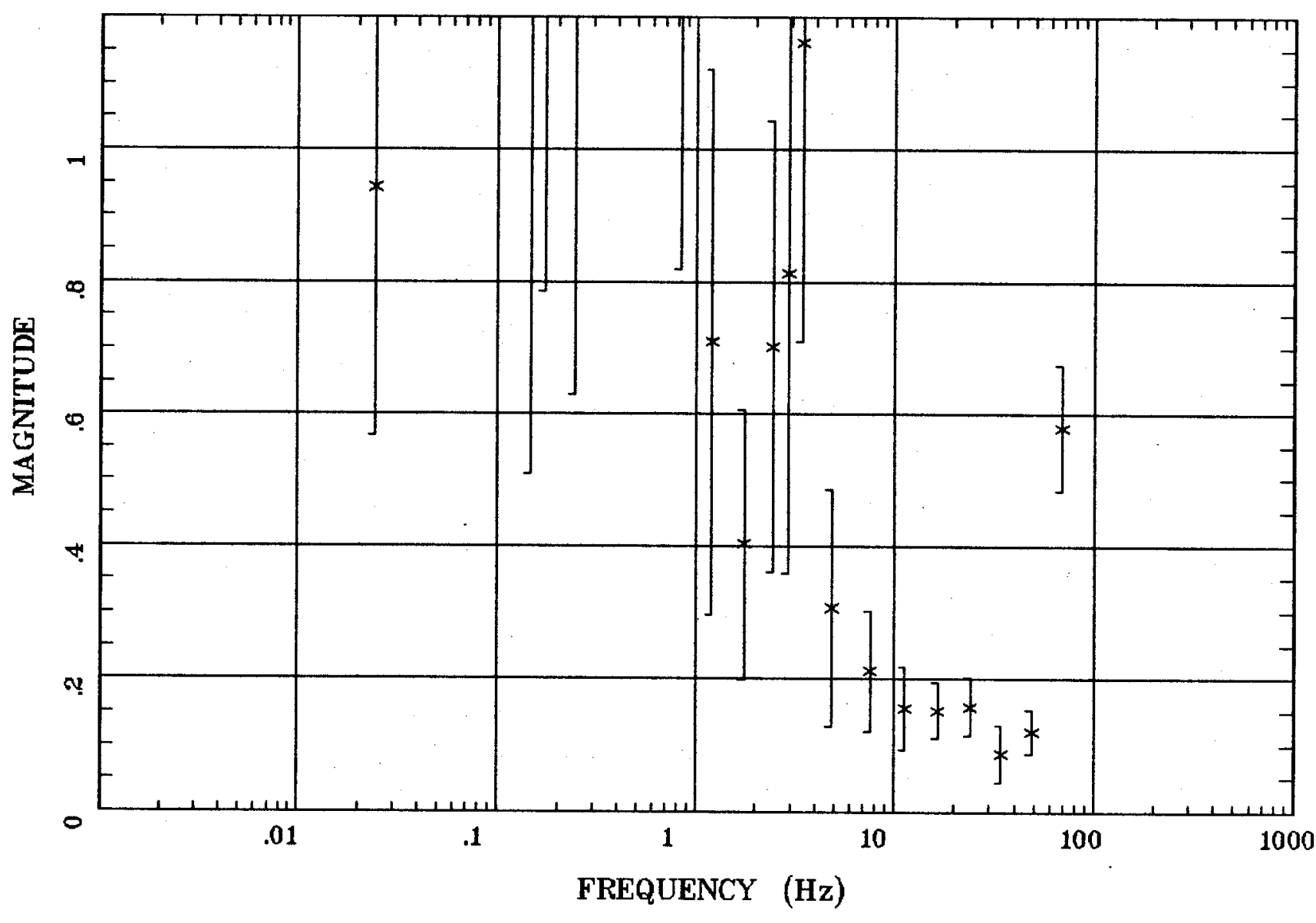

Client:

Remate: none

Acquired: 12:4 Aug 09, 1999

Survey Co:USGS
Rotation:

Filename: ar88b.avg

Channels: Ch1 Ch2 Ch3 Ch4 Ch5 Ch3 Ch4

Plotted: 11:07 Dec 08, 2000

< EMI - ElectroMagnetic Instruments 
TIPPER STRIKE

Kobeh Valley, NV

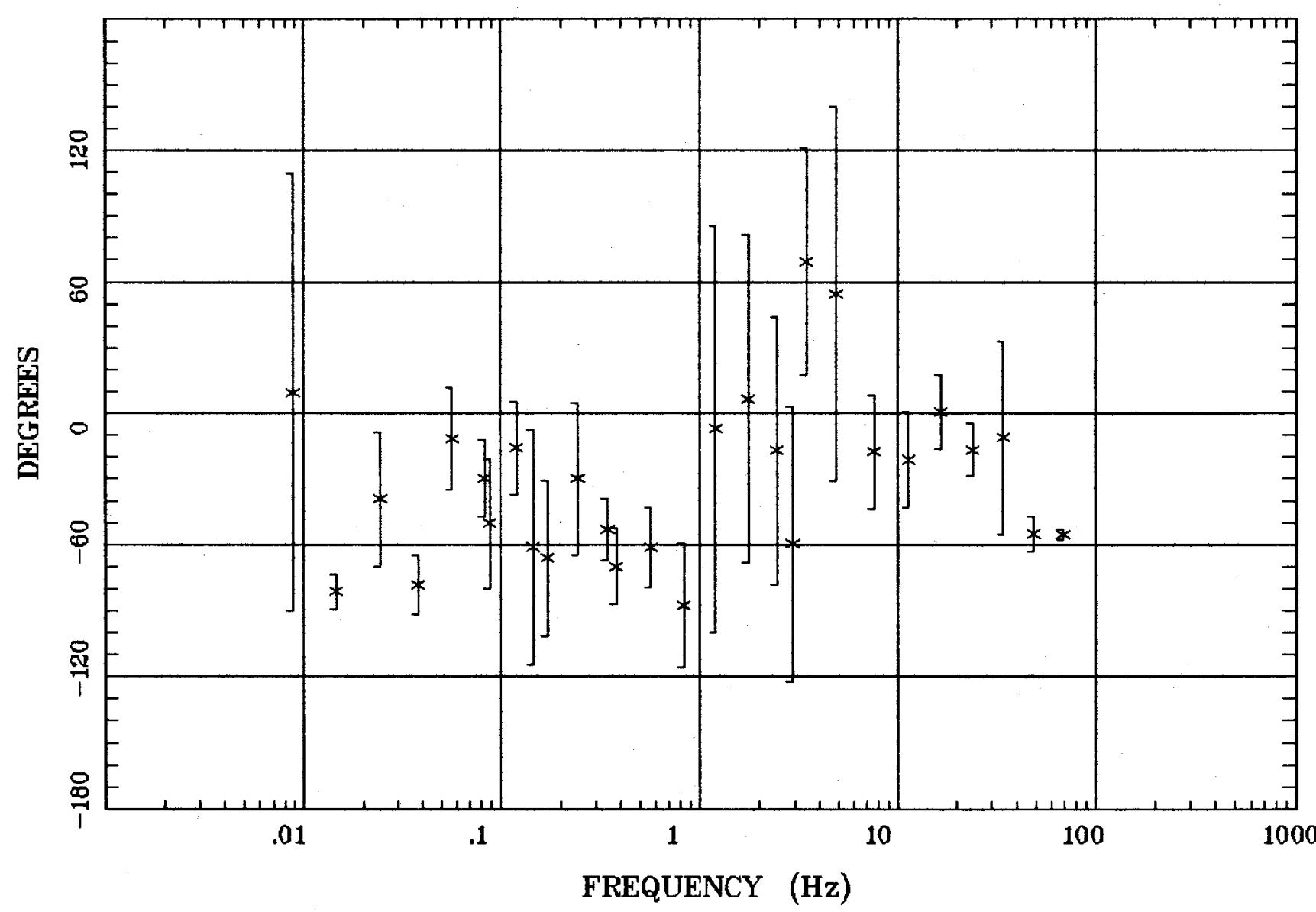

Client:

Remote: none

Acquired: 12:4 Aug 09, 1999 Survey Co:USGS
Rotation:

Filename: ar88b.avg

Channels: Ch1 Ch2 Ch3 Ch4 Ch5 Ch3 Ch4

Plotted: 11:07 Dec 08, 2000

< EMI - ElectroMagnetic Instruments 
Station 88

HzHx.x Coh HzHy.o Kobeh Valley, NV

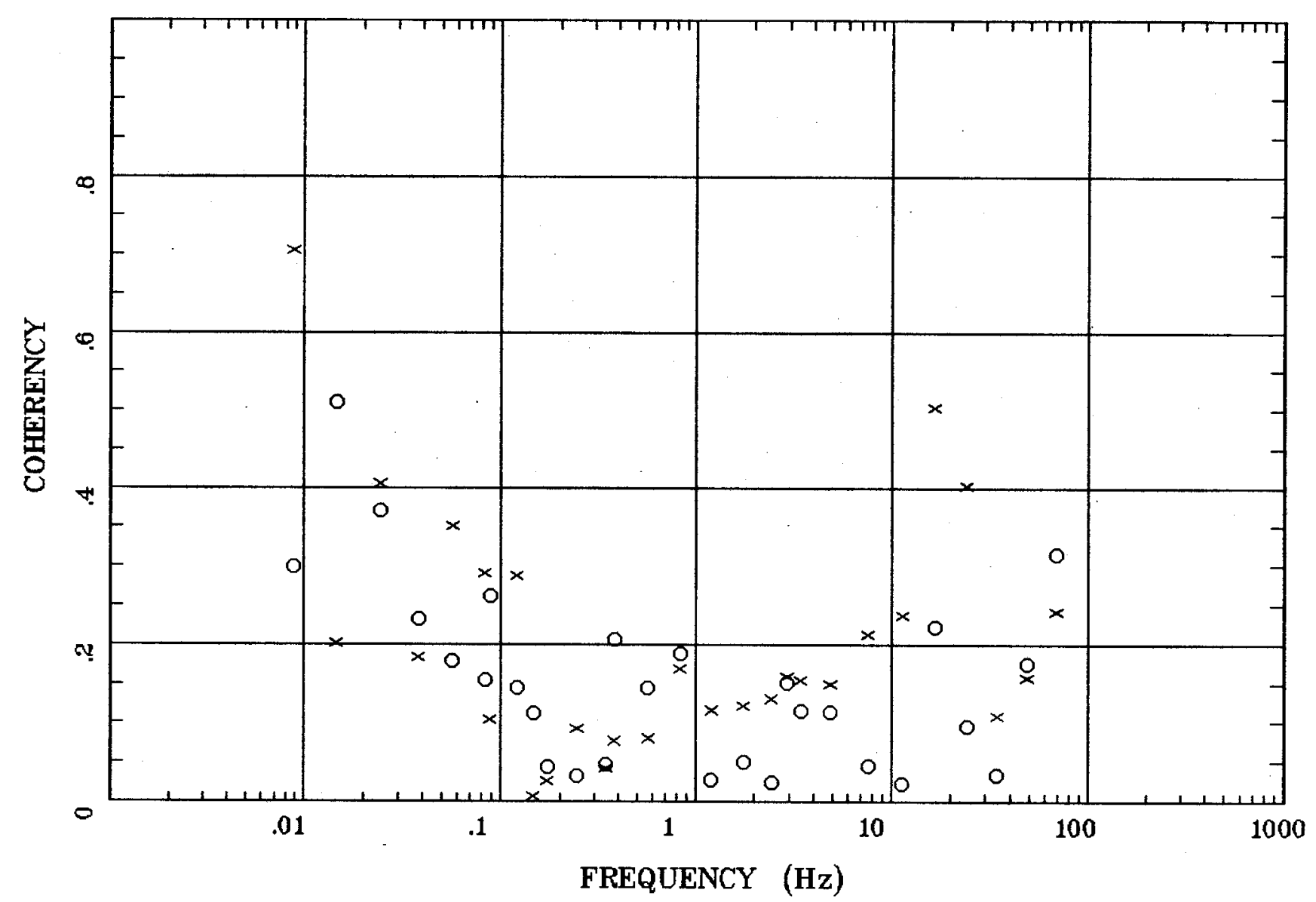

Client:

Remote: none

Acquired: 12:4 Aug 09, 1999

Survey Co:USGS
Rotation:

Filename: ar88b.avg

Channels: Ch1 Ch2 Ch3 Ch4 Ch5 Ch3 Ch4

Platted: 11:07 Dec 08, 2000

$<$ EMI - ElectroMagnetic Instruments 
Station 87

APPARENT RESISTIVITY

Kobeh Valley, NV

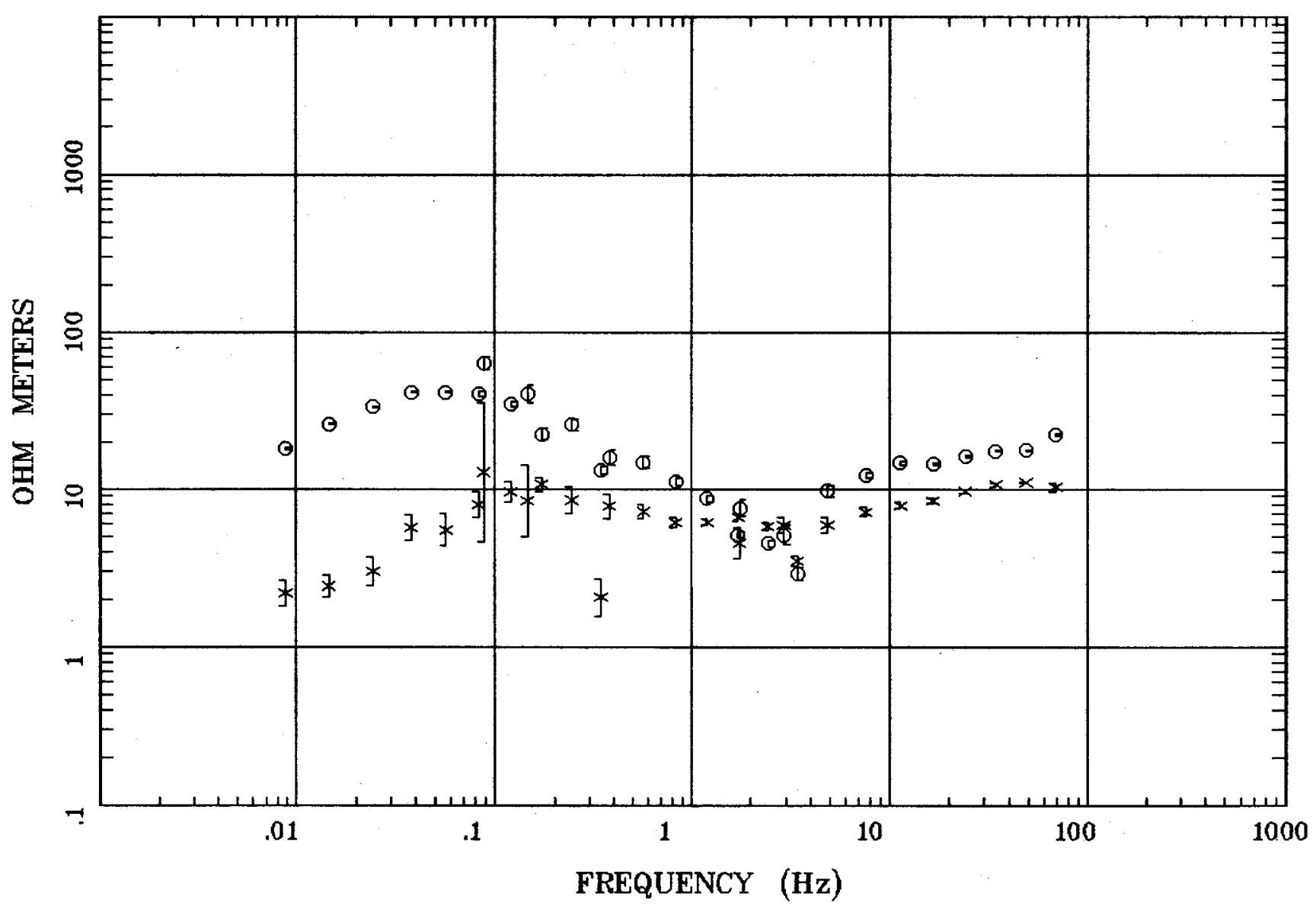

Client:

Remote: none

Acquired: 09:1 Aug 09, 1999 Survey Co:USGS
Rotation:

Filename: ar87all.avg

Channels: Ch1 Ch2 Ch3 Ch4 Ch5 Ch3 Ch4

Plotted: 11:05 Dec 08, 2000

< EMI - ElectroMagnetic Instruments > 
IMPEDANCE PHASE

Kobeh Valley, NV

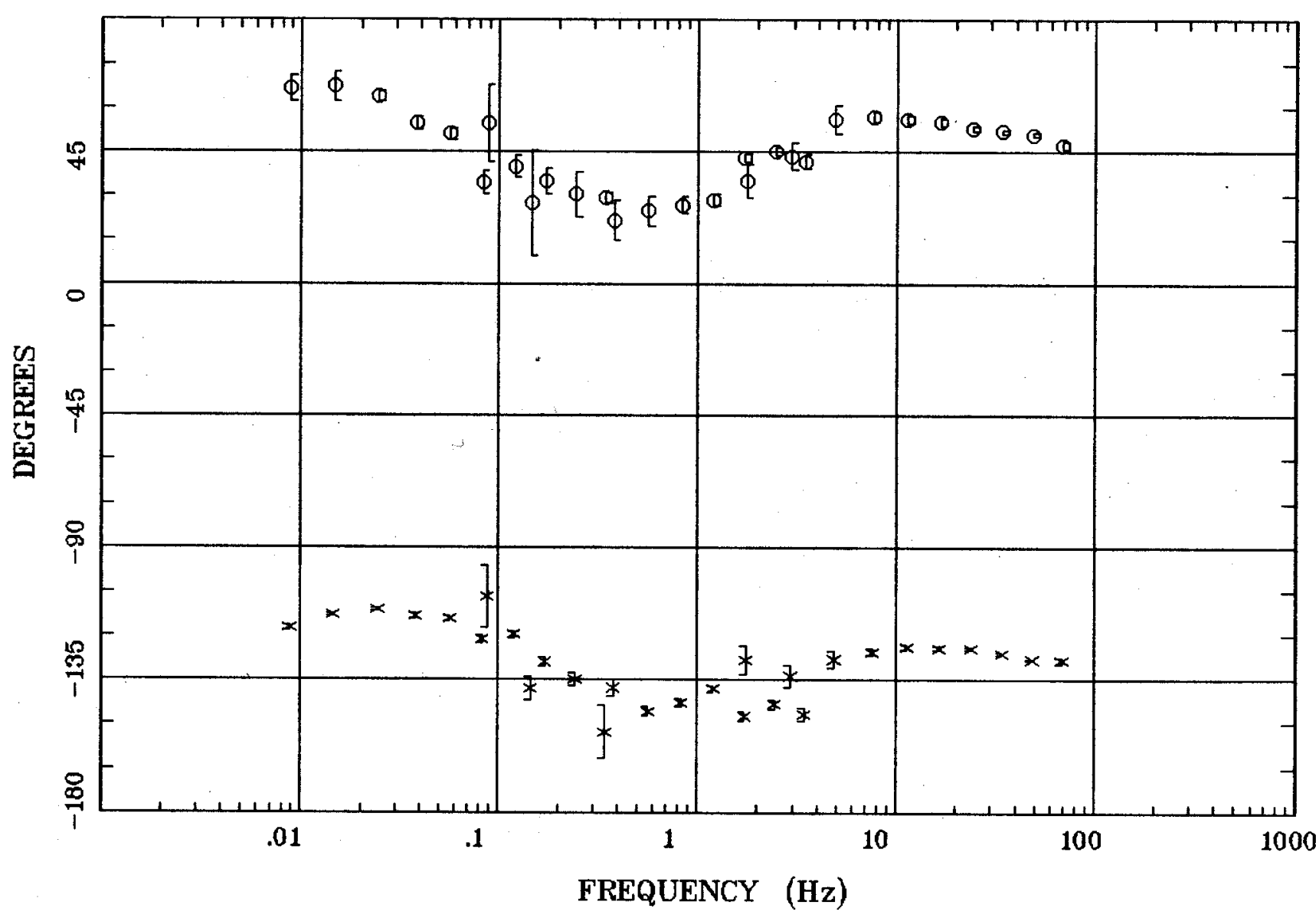

Client:

Remote: none

Acquired: 09:1 Aug 09, 1999 Survey Co:USGS

\section{Rotation:}

Filename: ar87all.avg

Channels: Ch1 Ch2 Ch3 Ch4 Ch5 Ch3 Ch4

Plotted: 11:05 Dec 08, 2000

< EMI - ElectroMagnetic Instruments > 
Station 87

ROTATION ANGLE

Kobeh Valley, NV

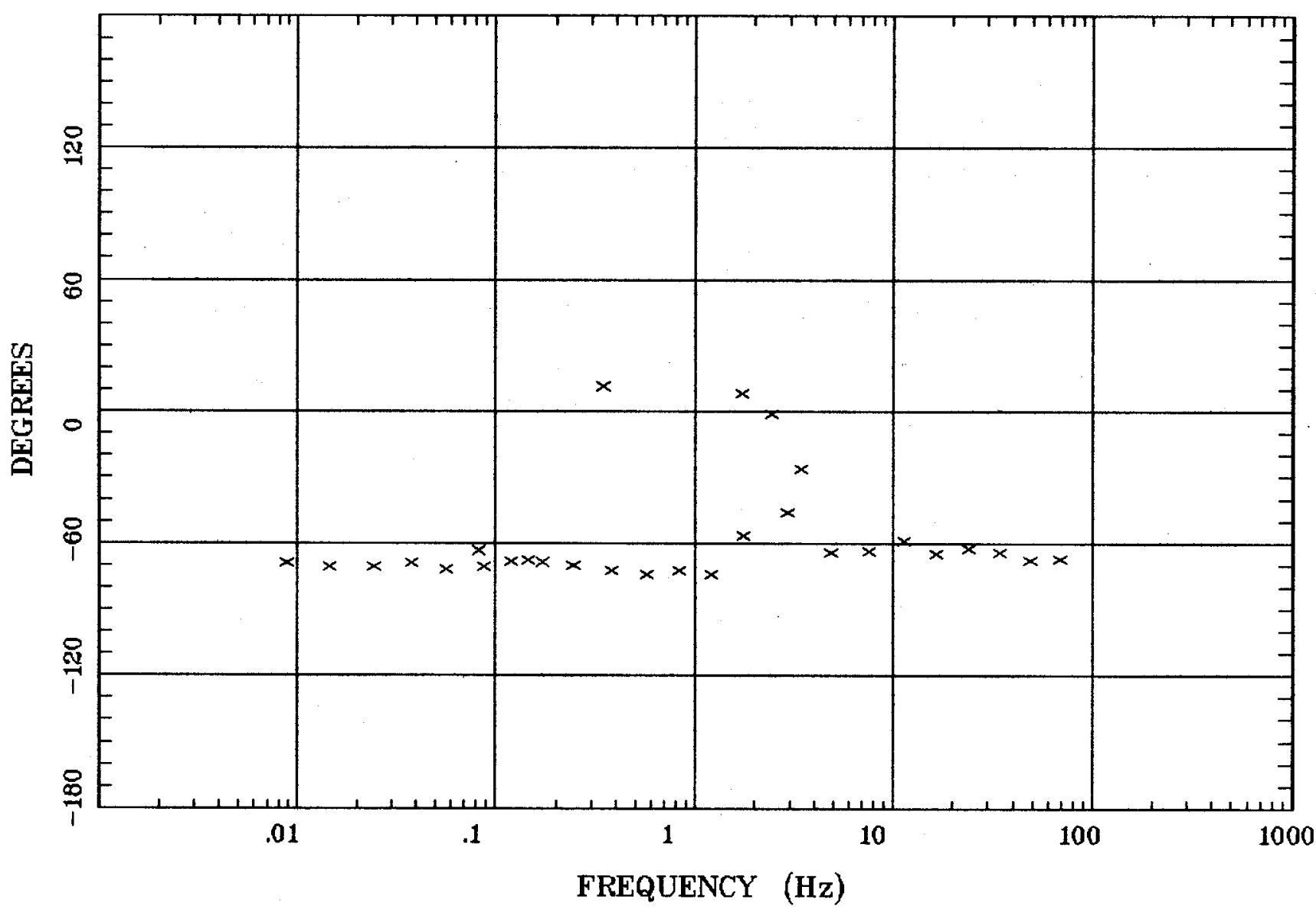

Client:

Remote: none

Acquired: 09:1 Aug 09, 1999

Survey Co:USGS
Rotation:

Filename: ar87all.avg

Channels: Ch1 Ch2 Ch3 Ch4 Ch5 Ch3 Ch4

Plotted: 11:05 Dec 08, 2000

< EMI - ElectroMagnetic Instruments 


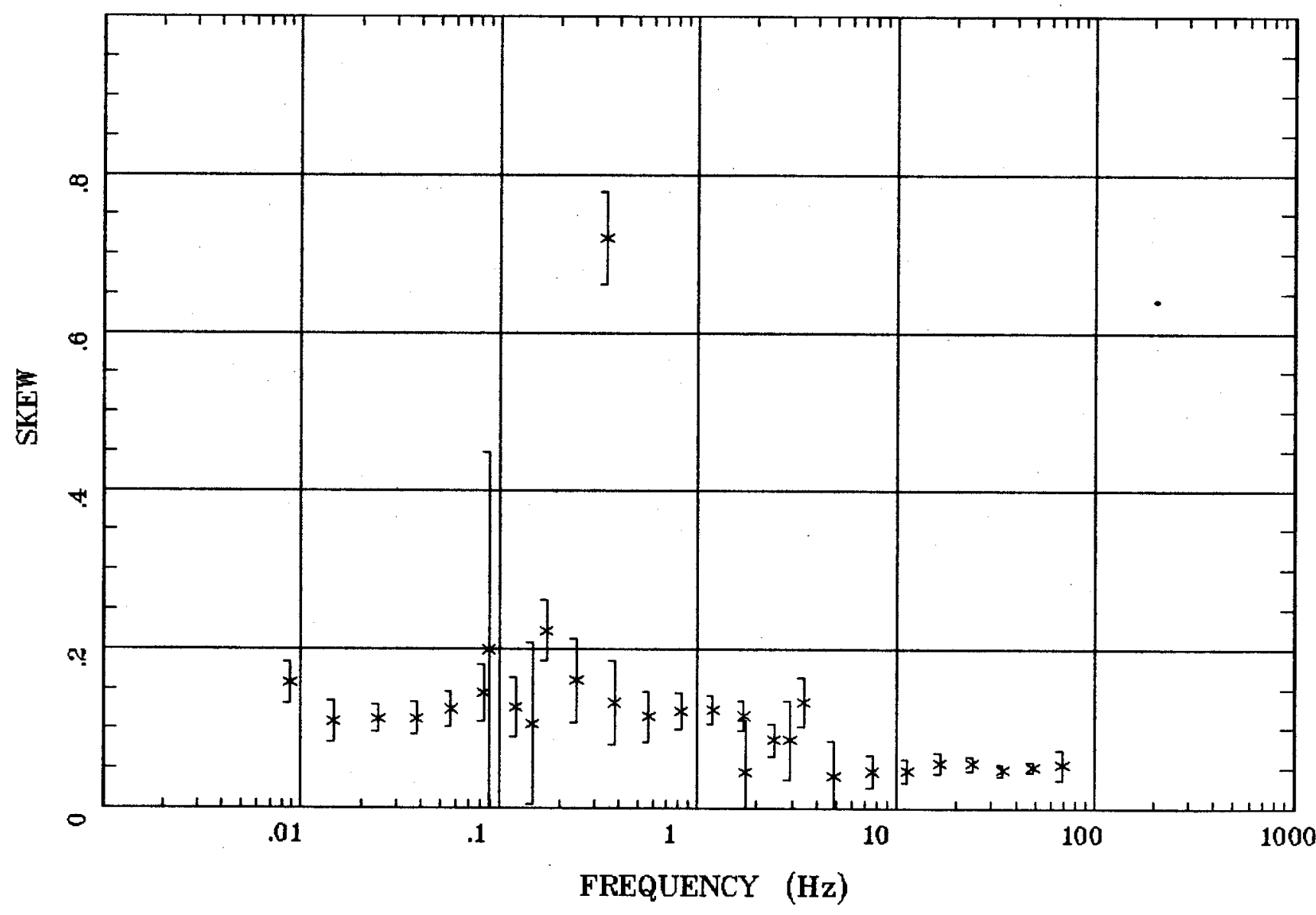

Client:

Remote: none Acquired: 09:1 Aug 09, 1999 Survey Co:USGS
Rotation:

Filename: ar87all.avg

Channels: Ch1 Ch2 Ch3 Ch4 Ch5 Ch3 Ch4

Plotted: 11:06 Dec 08, 2000

< EMI - ElectroMagnetic Instruments 


\section{Station 87}

E MULT Coh.

Kobeh Valley, NV

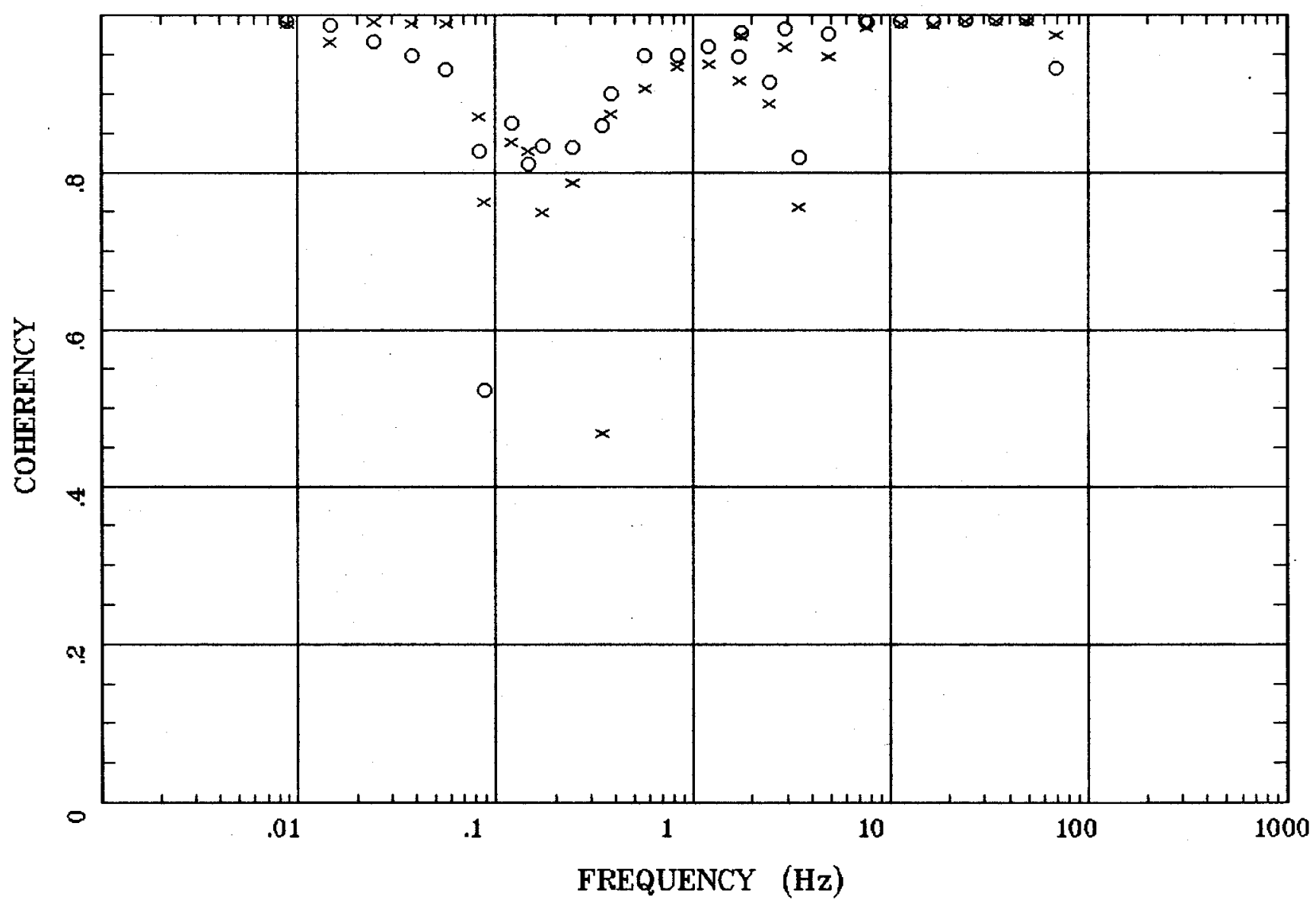

Client:

Rotation:

Filename: ar87all.avg

Remote: none

Channels: Ch1 Ch2 Ch3 Ch4 Ch5 Ch3 Ch4

Acquired: 09:1 Aug 09, 1999

Plotted: 11:06 Dec 08, 2000

Survey Ca:USGS

< EMI - ElectroMagnetic Instruments 


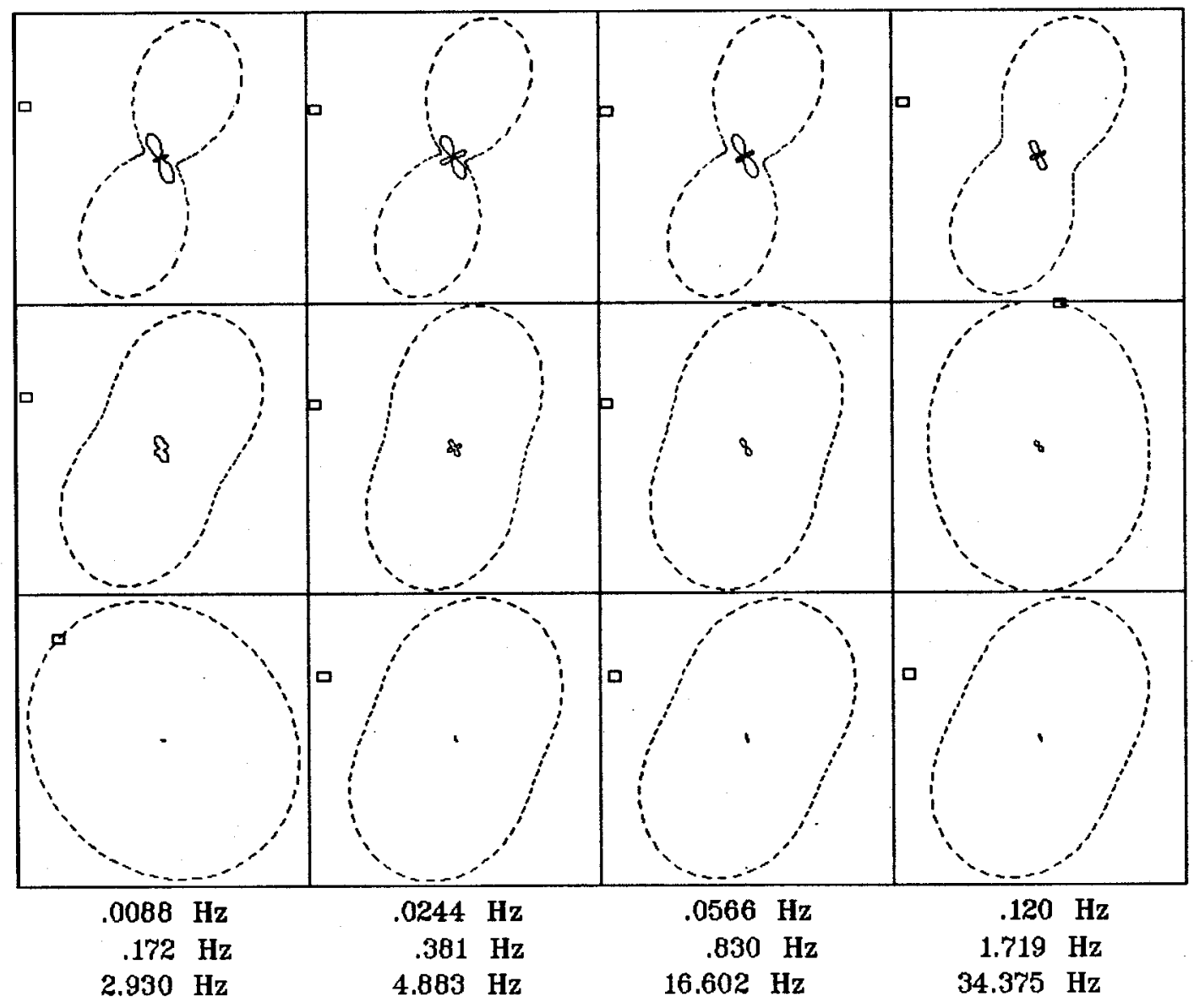

Client:

Remote: none

Acquired: 09:1 Aug 09, 1999 Survey Co:USGS
Rotation:

Filename: ar87all.avg

Channels: Ch1 Ch2 Ch3 Ch4 Ch5 Ch3 Ch4 Plotted: 11:06 Dec 08, 2000

$<$ EMI - ElectroMagnetic Instruments 


\section{Station 87}

TIPPER MAGNITUDE

Kobeh Valley, NV

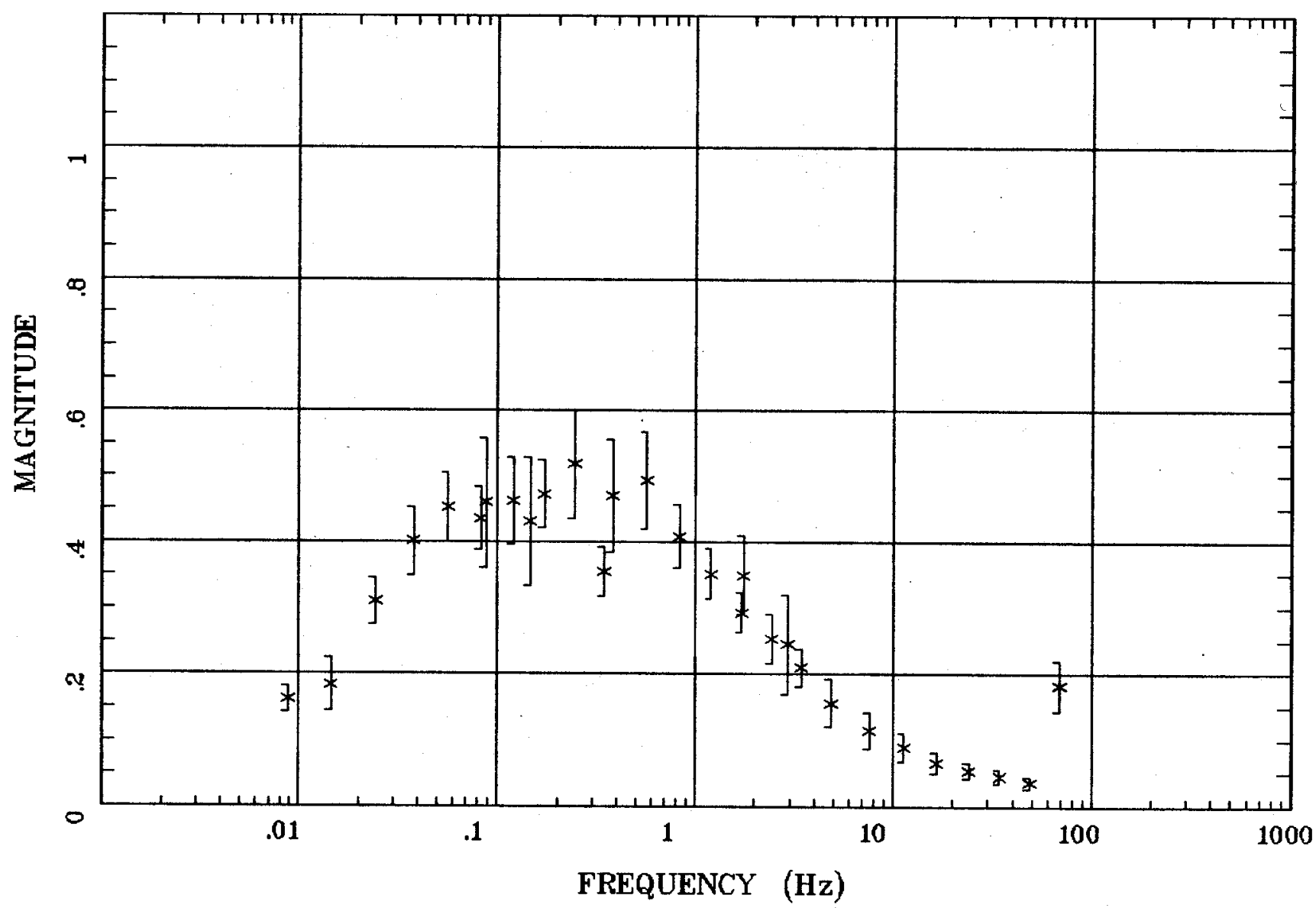

Client:

Rotation:

Remote: none

Filename: ar87all.avg

Acquired: 09:1 Aug 09, 1999

Channels: Ch1 Ch2 Ch3 Ch4 Ch5 Ch3 Ch4

Plotted: 11:06 Dec 08, 2000

Survey Co:USGS

< EMI - ElectroMagnetic Instruments 


\section{Station 87}

TIPPER STRIKE

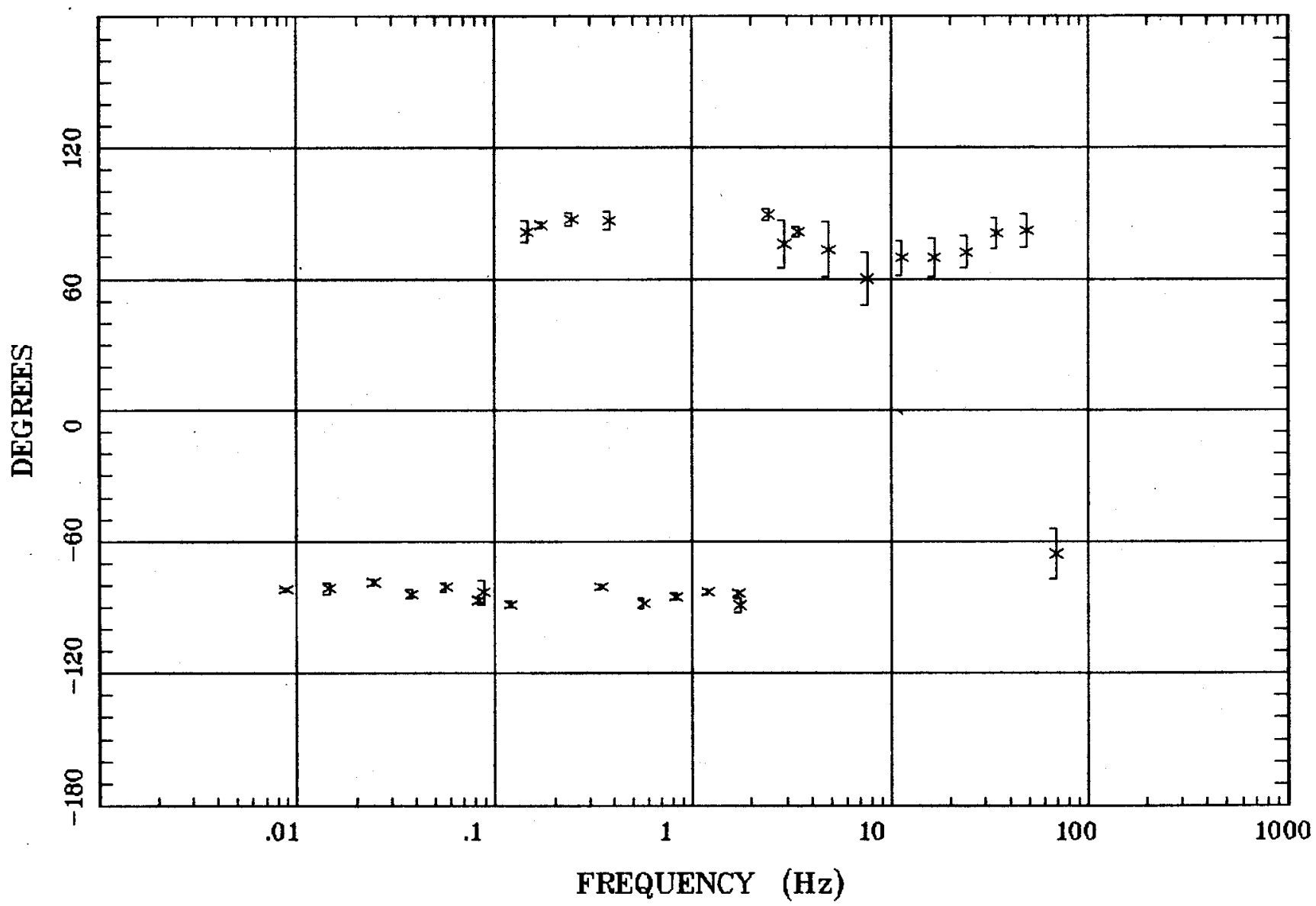

Client:

Remote: none

Acquired: 09:1 Aug 09, 1999

Survey Co:USGS
Kobeh Valley, NV

Rotation:

Filename: ar87all.avg

Channels: Ch1 Ch2 Ch3 Ch4 Ch5 Ch3 Ch4

Plotted: 11:06 Dec 08, 2000

< EMI - ElectroMagnetic Instruments > 


\section{HzHx.x Coh HzHy.o}

Kobeh Valley, NV

Station 87

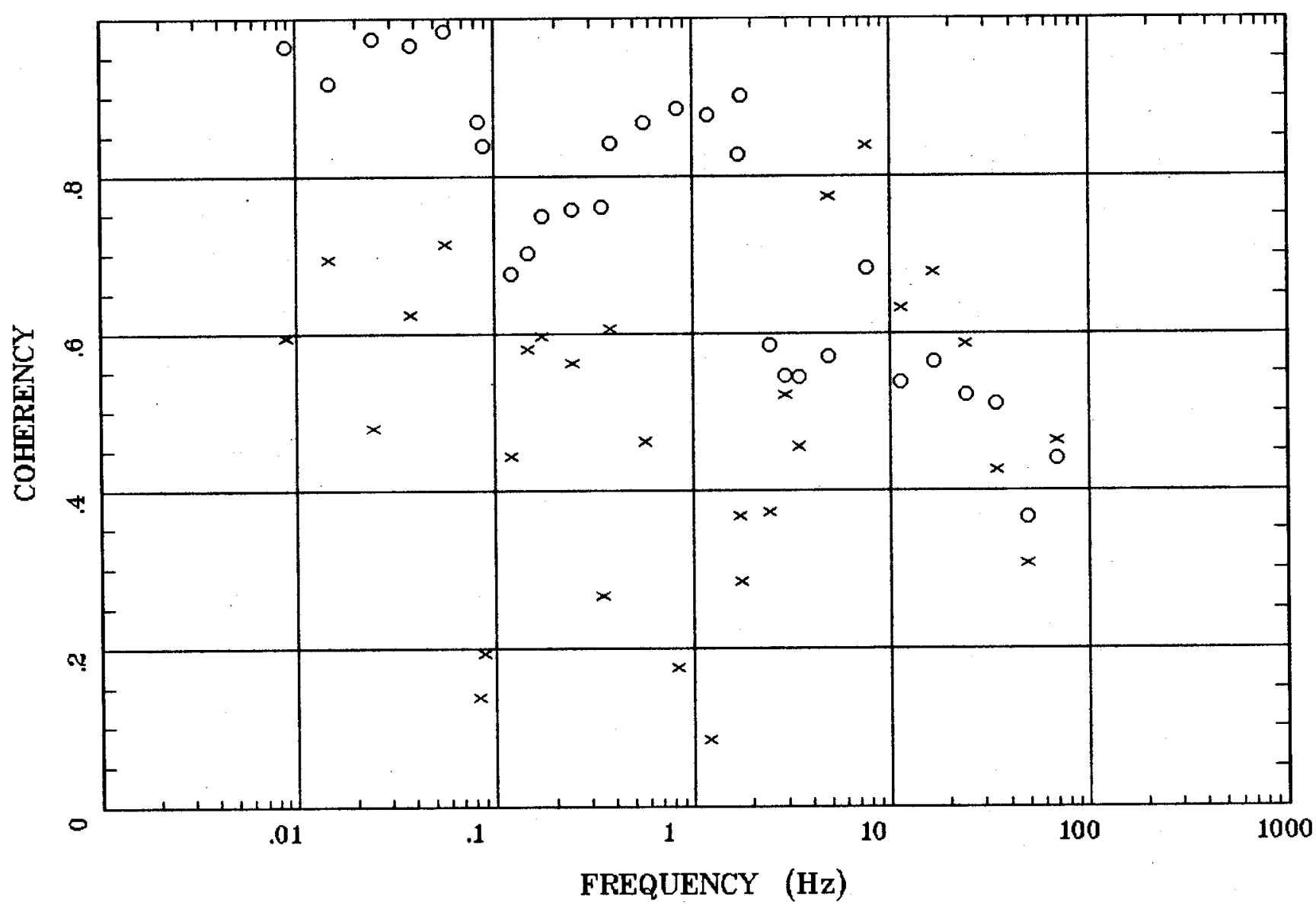

Client:

Remote: none

Acquired: 09:1 Aug 09, 1999 Survey Co:USGS
Rotation:

Filename: ar87all.avg

Channels: Ch1 Ch2 Ch3 Ch4 Ch5 Ch3 Ch4 Plotted: 11:06 Dec 08, 2000

< EMI - ElectroMagnetic Instruments 


\section{APPARENT RESISTIVITY}

Kobeh Valley, NV

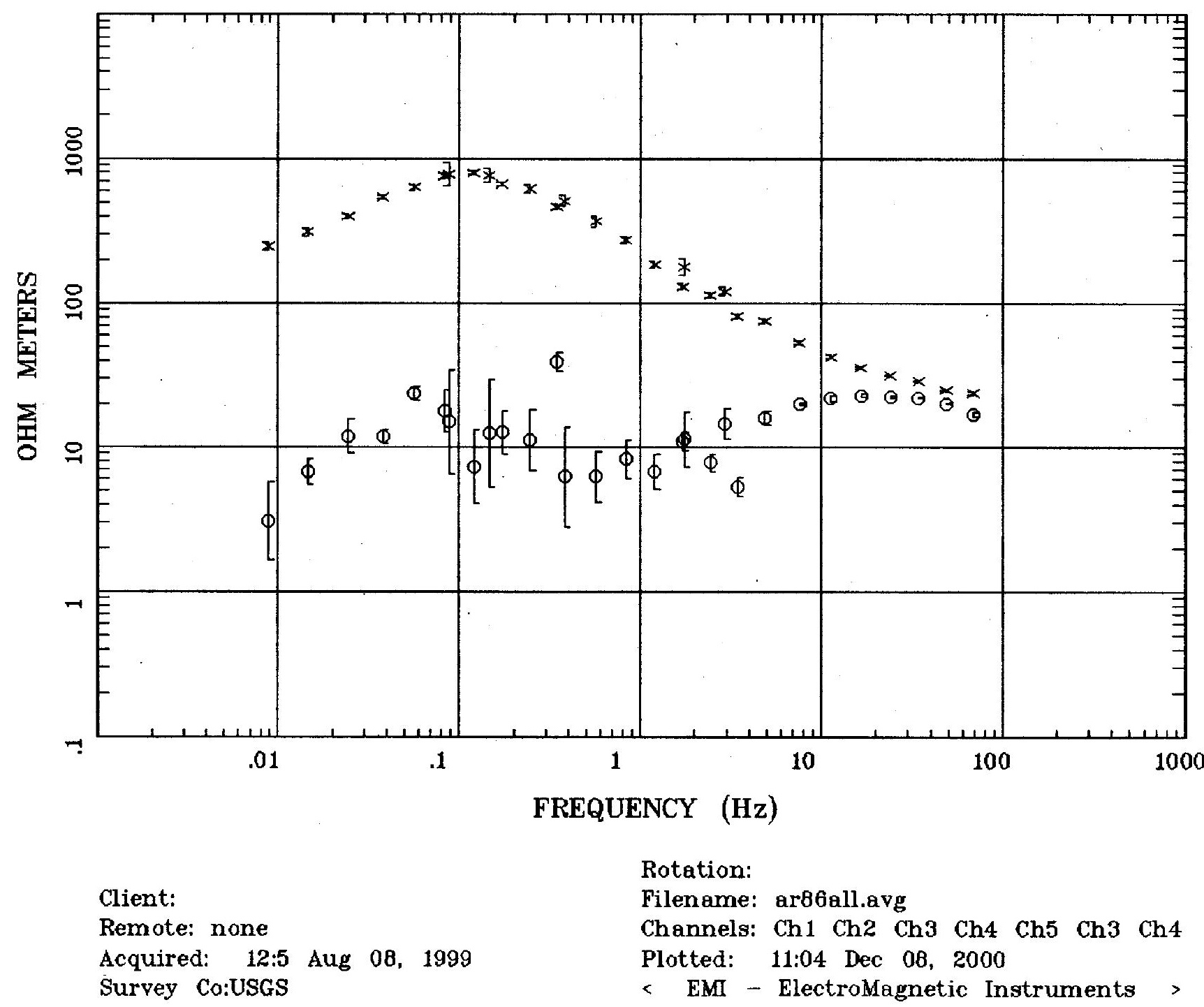


IMPEDANCE PHASE

Kobeh Valley, NV

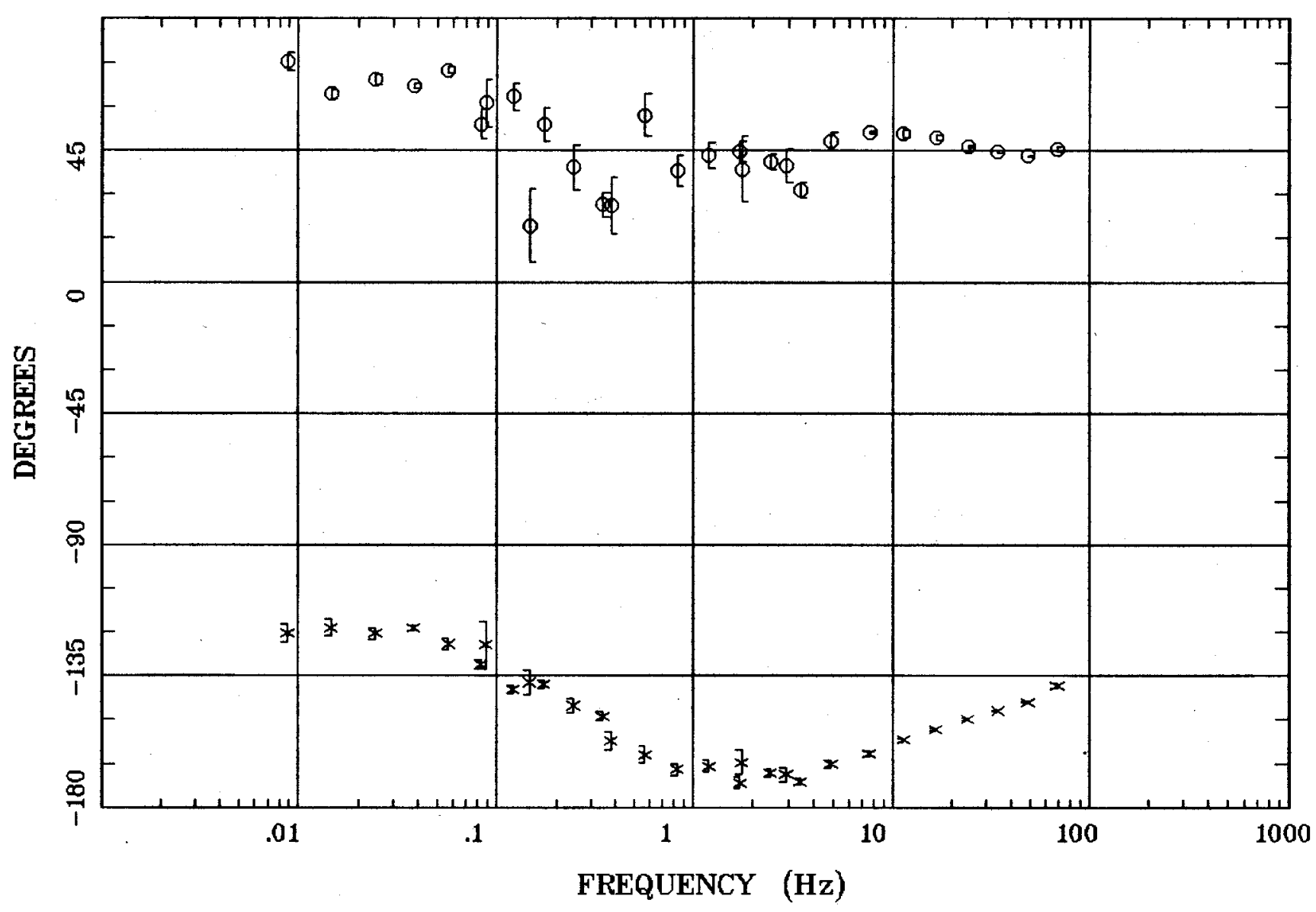

Client:

Remote: none

Acquired: 12:5 Aug 08, 1999 Survey Co:USGS
Rotation:

Filename: ar86all.avg

Channels: Ch1 Ch2 Ch3 Ch4 Ch5 Ch3 Ch4

Plotted: 11:04 Dec 08, 2000

< EMI - ElectroMagnetic Instruments 


\section{Station 86}

ROTATION ANGLE

Kobeh Valley, NV

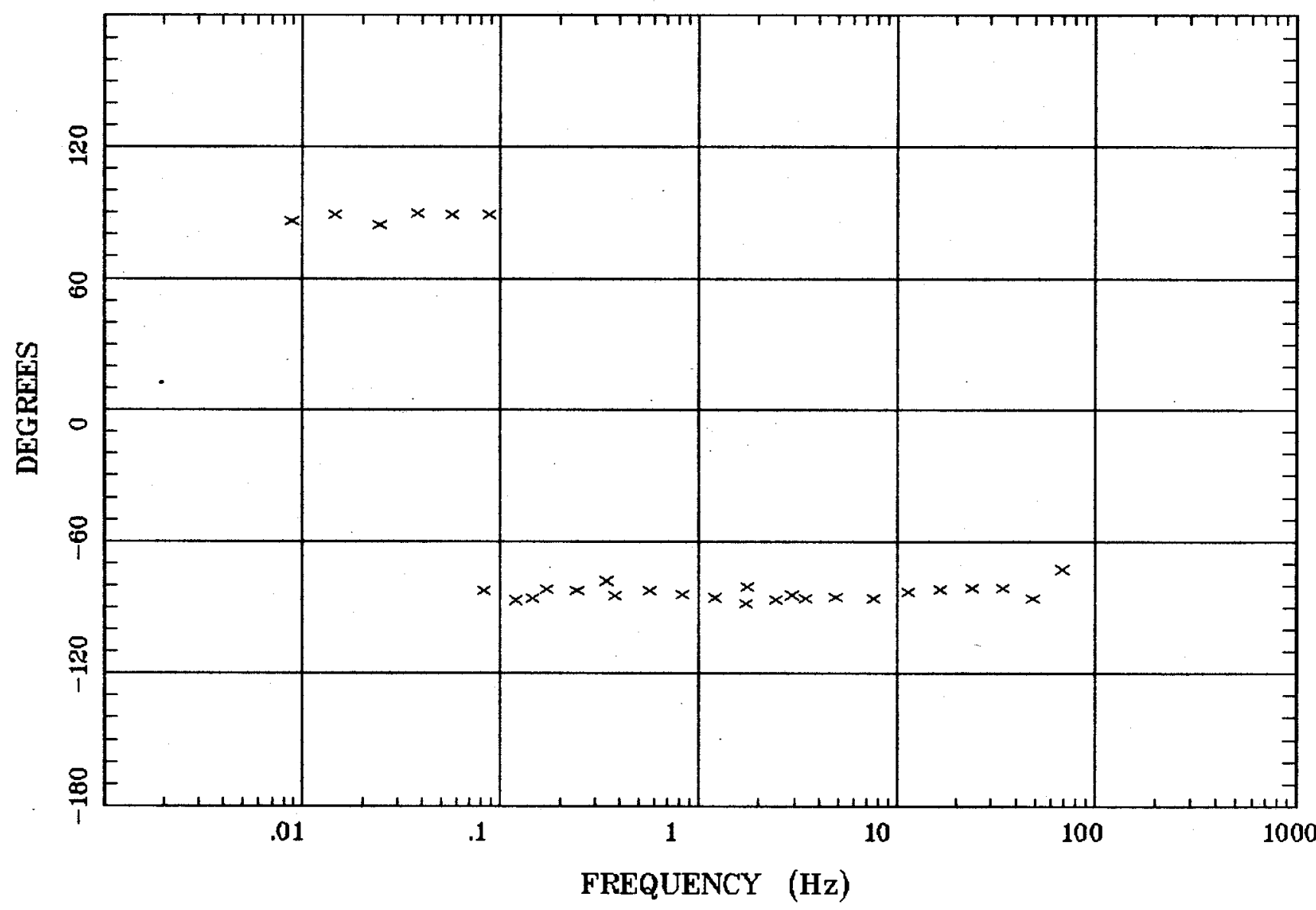

Client:

Remote: none

Acquired: 12:5 Aug 08, 1999

Survey Co:USGS
Rotation:

Filename: ar86all.avg

Channels: Ch1 Ch2 Ch3 Ch4 Ch5 Ch3 Ch4 Plotted: 11:04 Dec 08, 2000

< EMI - ElectroMagnetic Instruments 
IMPEDANCE SKEW

Kobeh Valley, NV

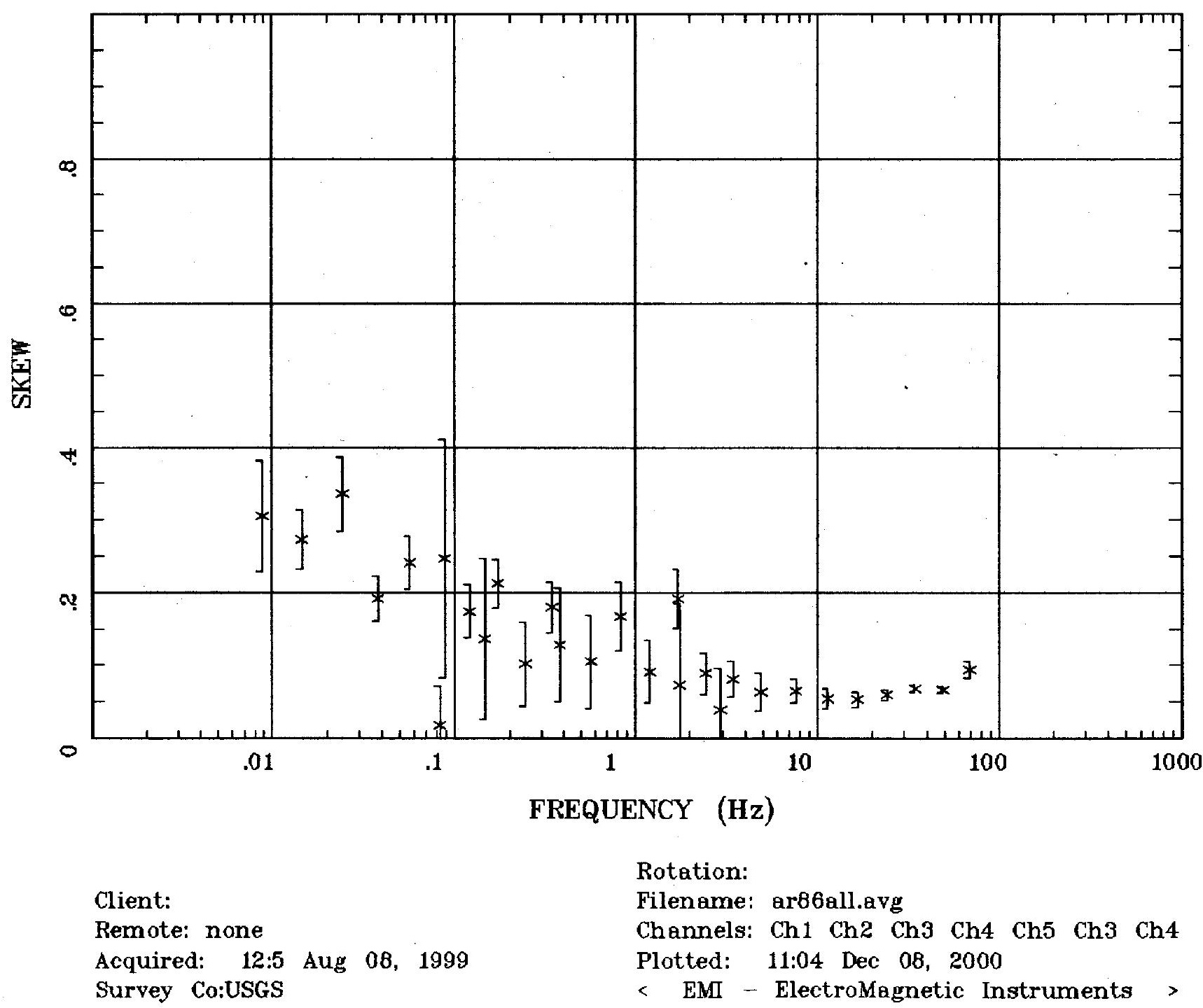


E MULT Coh.

Kobeh Valley, NV

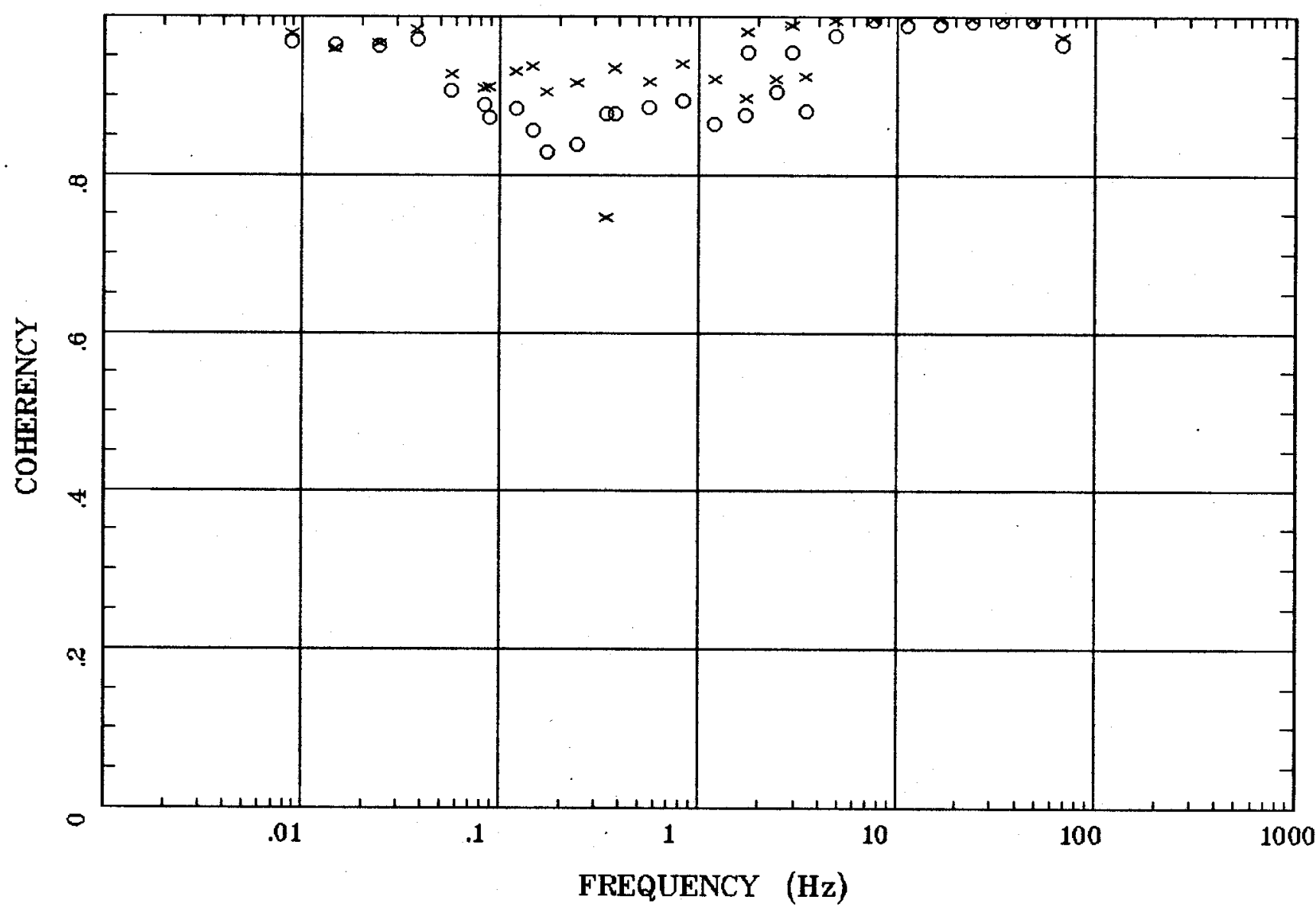

Client:

Remote: none

Acquired: 12:5 Aug 08, 1999

Survey Co:USGS
Rotation:

Filename: ar86all.avg

Channels: Ch1 Ch2 Ch3 Ch4 Ch5 Ch3 Ch4

Plotted: 11:04 Dec 08, 2000

< EMI - ElectroMagnetic Instruments > 
Station 86

POLAR PLOTS

\section{Kobeh Valley, NV}

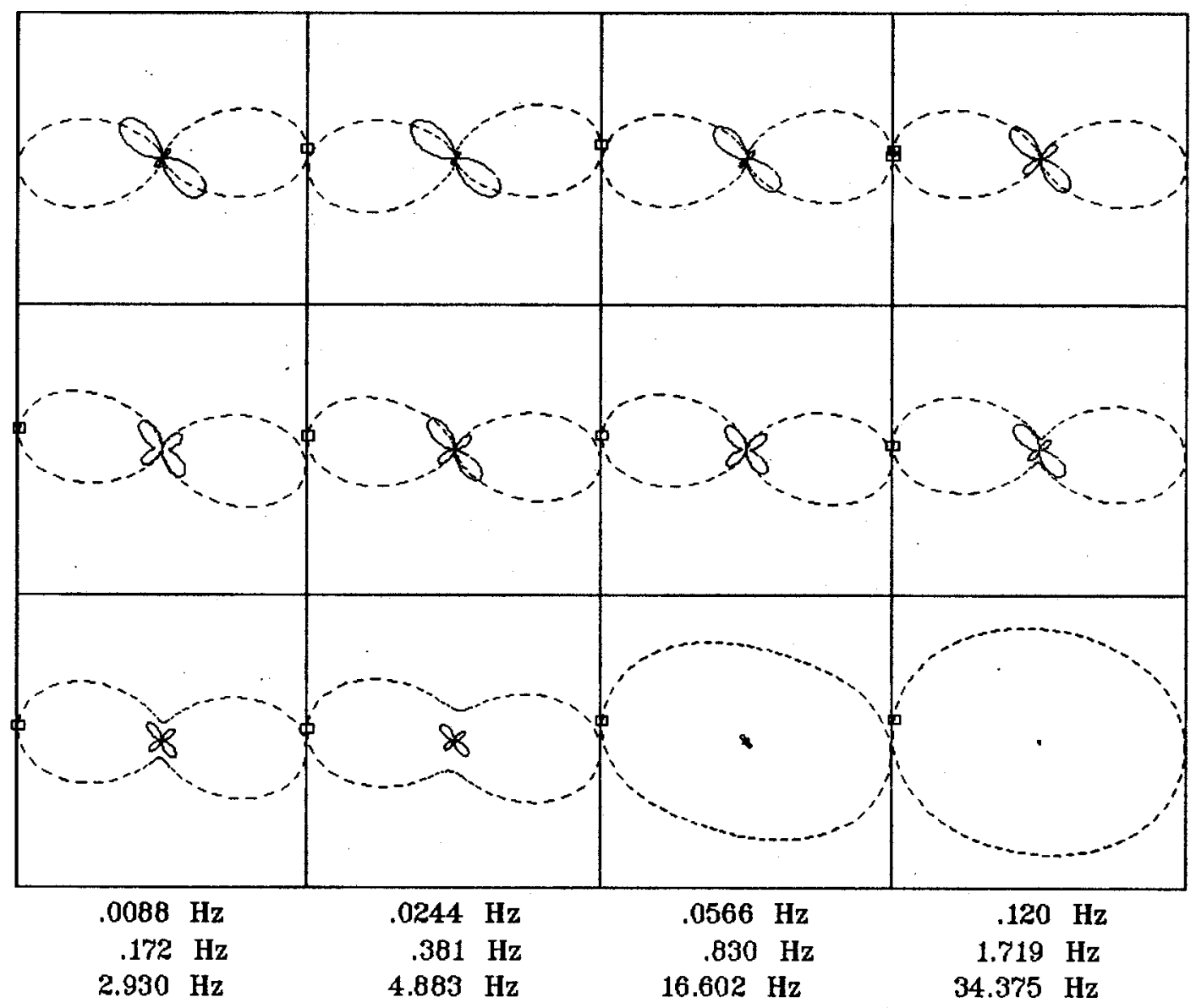

Client:

Remote: none

Acquired: 12:5 Aug 08, 1999 Survey Co:USGS
Rotation:

Filename: ar86all.avg

Channels: Ch1 Ch2 Ch3 Ch4 Ch5 Ch3 Ch4

Plotted: 11:04 Dec 08, 2000

$\langle$ EMI - ElectroMagnetic Instruments > 


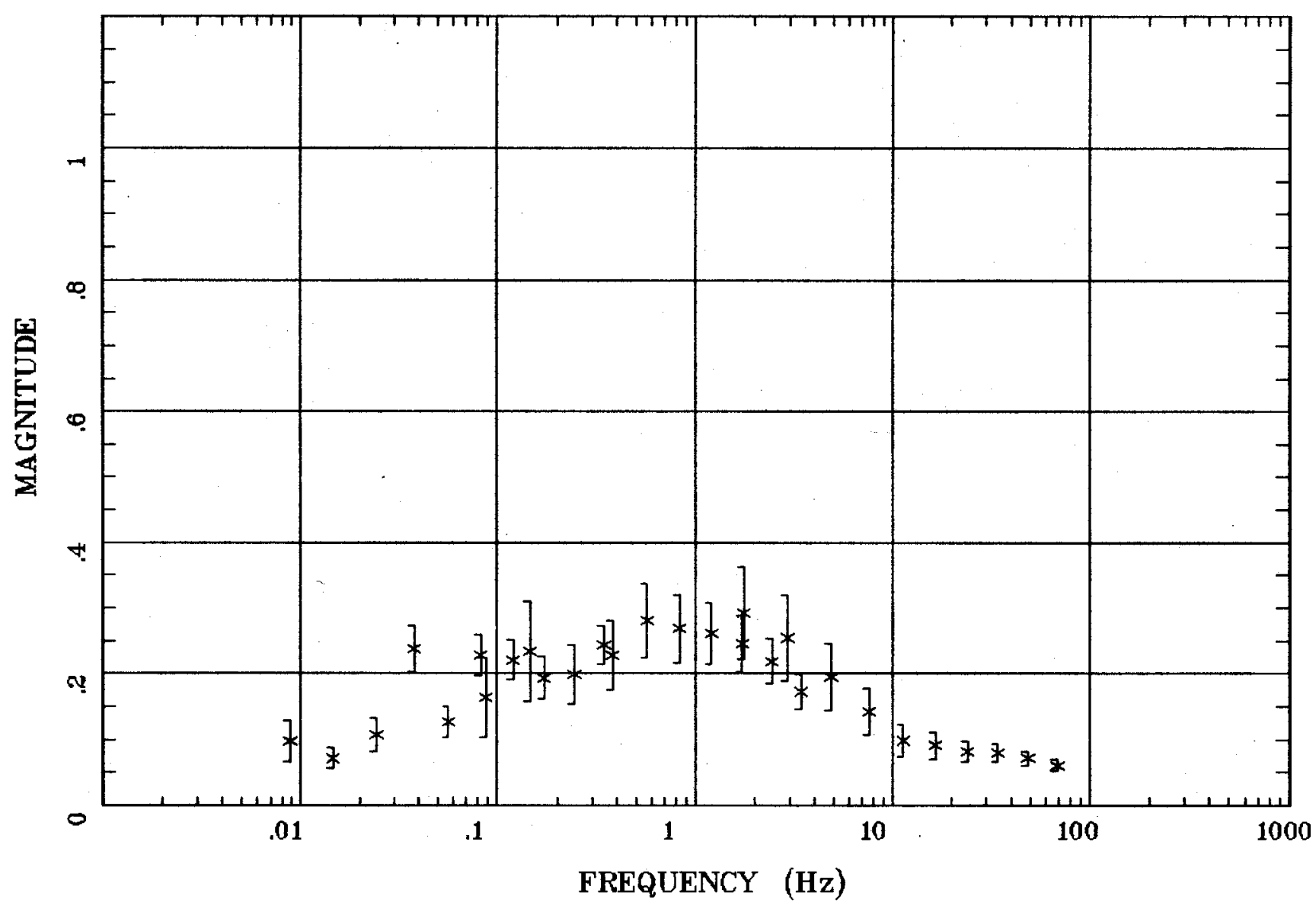

Client:

Remote: none

Acquired: 12:5 Aug 08, 1999

Survey Co:USGS
Rotation:

Filename: ar66all.avg

Channels: Ch1 Ch2 Ch3 ch4 Ch5 Ch3 Ch4 Platted: 11:04 Dec 08, 2000

$<$ EMI - ElectroMagnetic Instruments > 


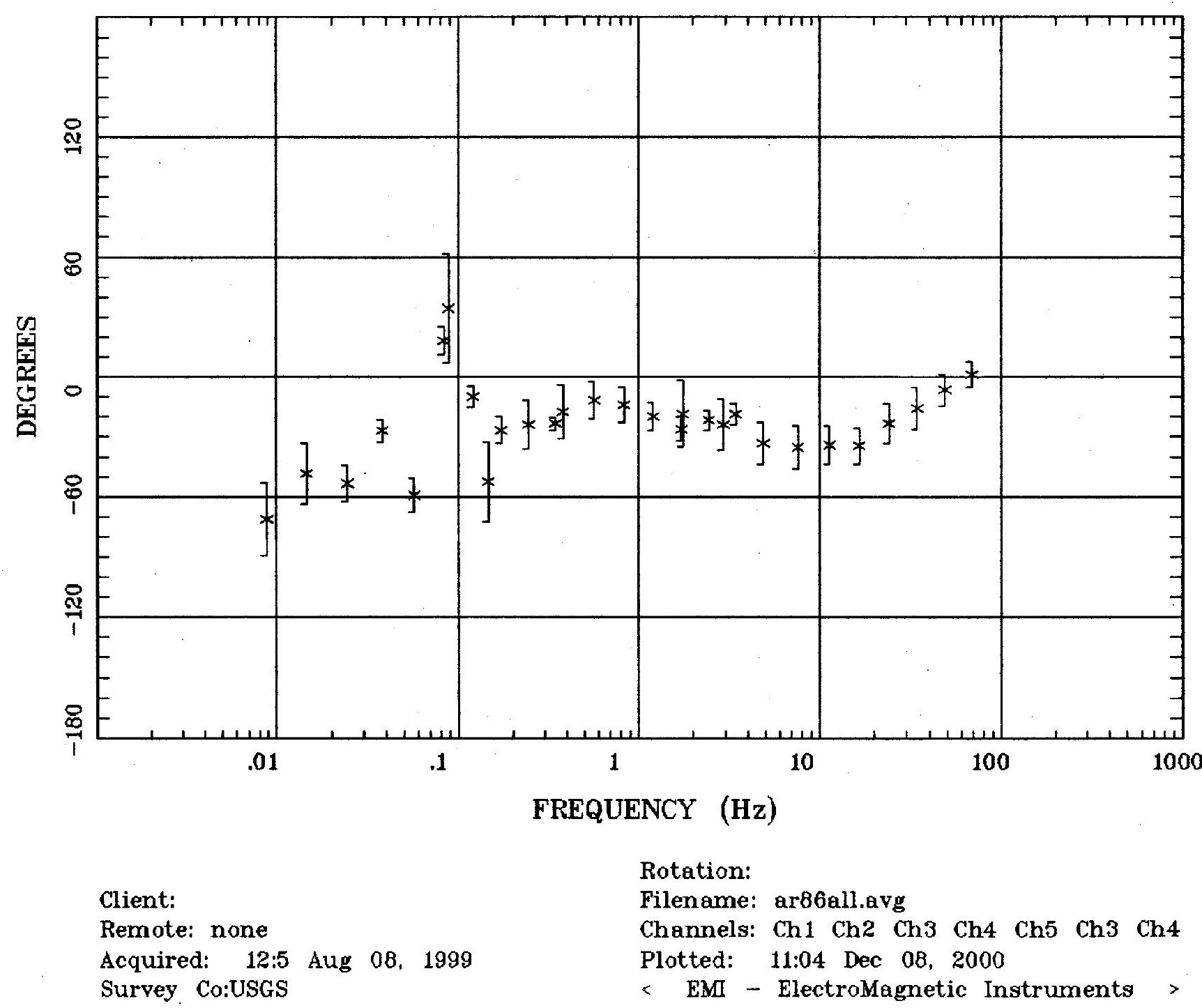


HzHx.x Coh HzHy.o

Kobeh Valley, NV

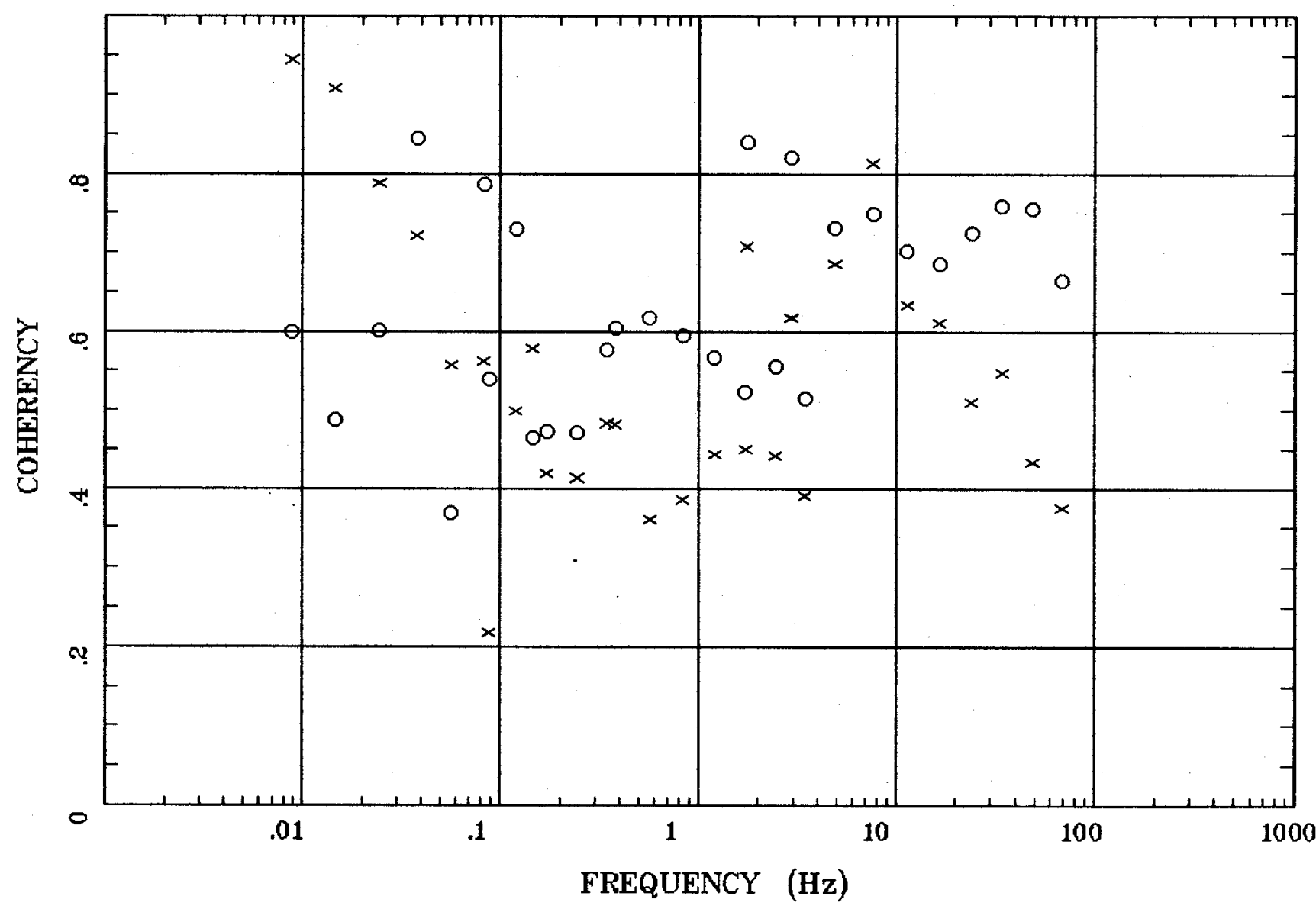

Client:

Remote: none

Acquired: 12:5 Aug 08, 1999 Survey Co:USGS
Rotation:

Filename: ar86all.avg

Channels: Ch1 Ch2 Ch3 Ch4 Ch5 Ch3 Ch4 Plotted: 11:04 Dec 08, 2000

< EMI - ElectroMagnetic Instruments > 
APPARENT RESISTIVITY

whistler mtn.,nv

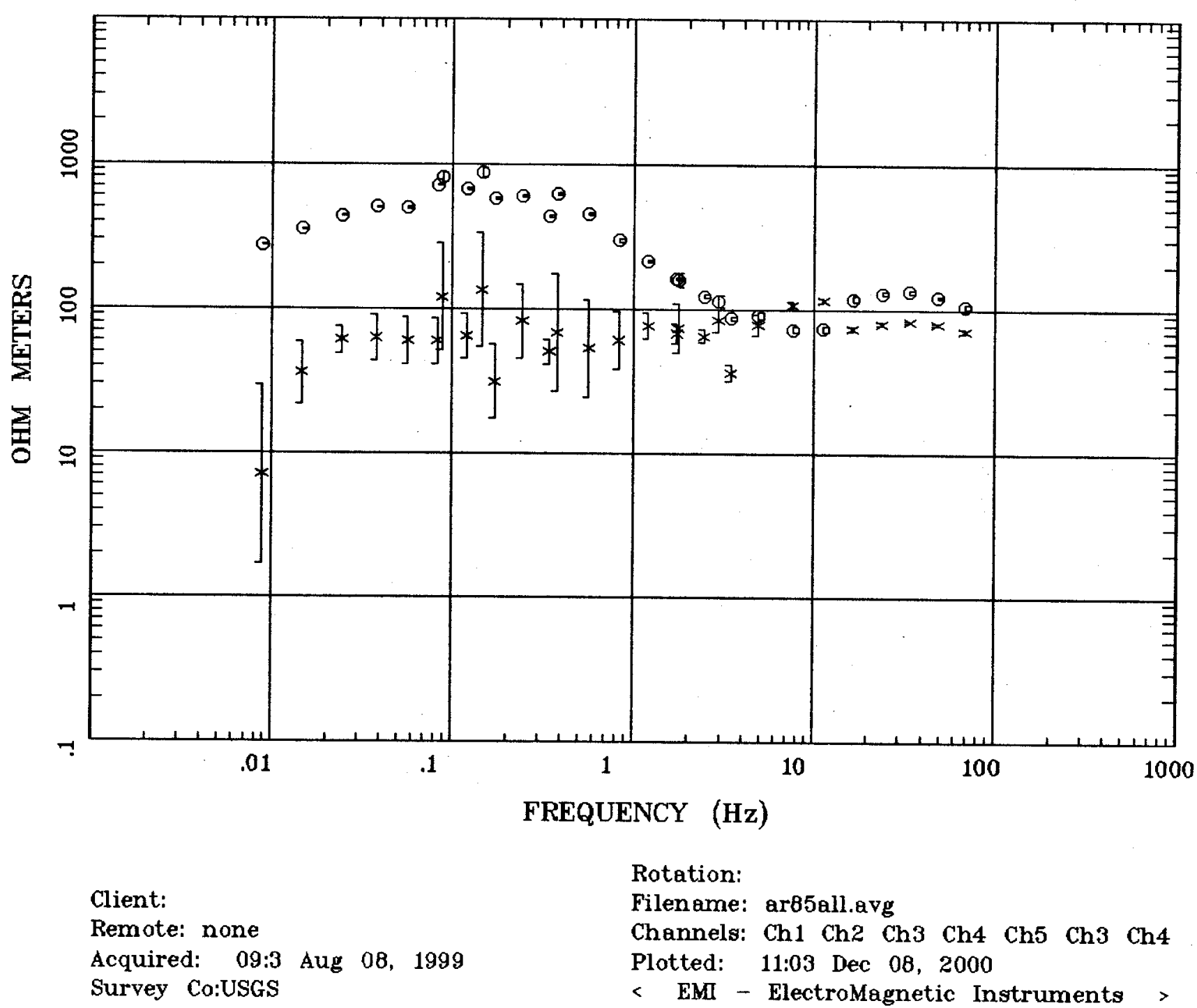


IMPEDANCE PHASE

whistler mtn.,nv

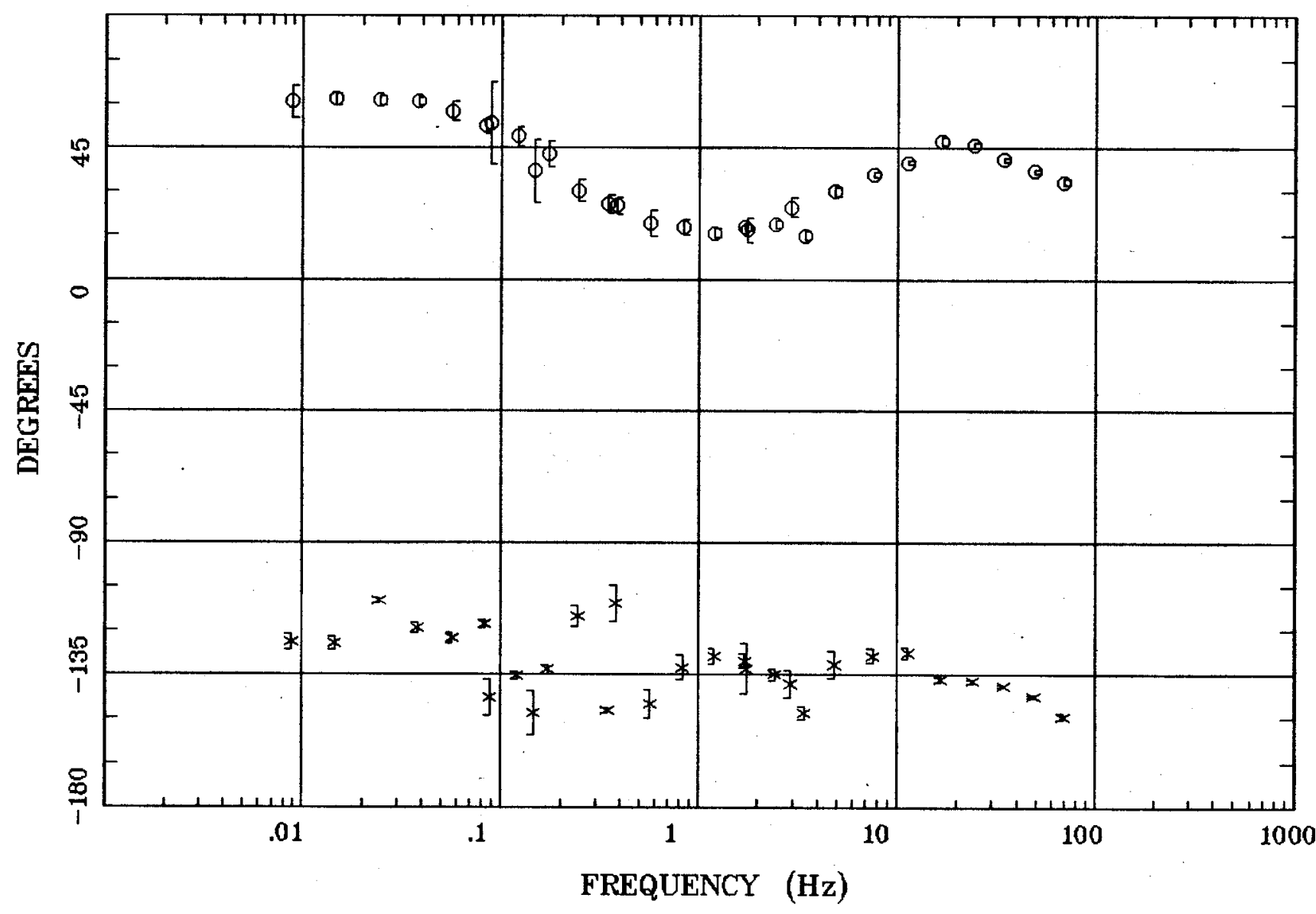

Client:

Remote: none

Acquired: 09:3 Aug 08, 1999

Survey Co:USGS
Rotation:

Filename: ar85all.avg

Channels: Ch1 Ch2 Ch3 Ch4 Ch5 Ch3 Ch4

Plotted: 11:03 Dec 08, 2000

$<$ EMI - ElectroMagnetic Instruments 


\section{ROTATION ANGLE}

whistler mtn.,nv

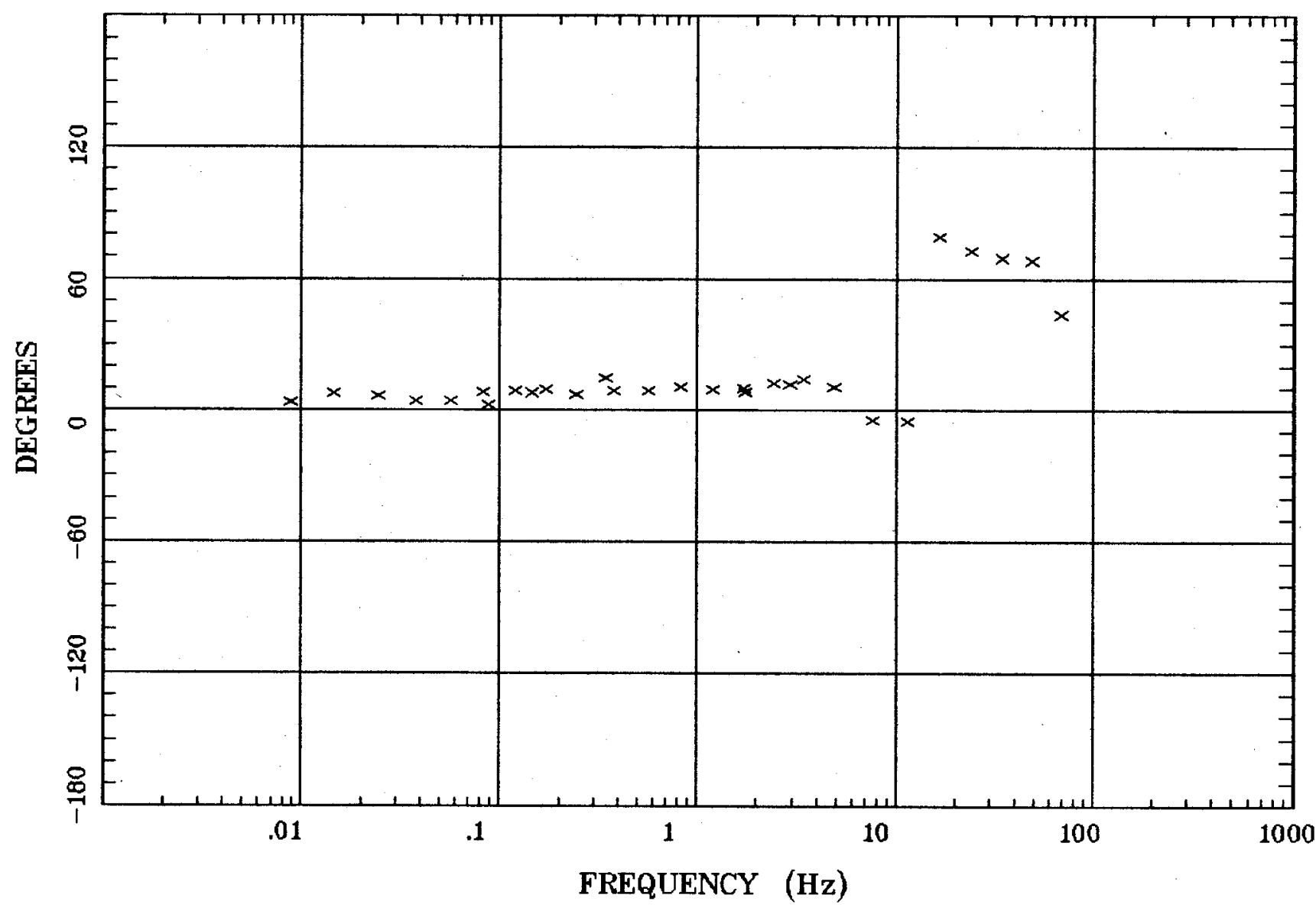

Client:

Remote: none

Acquired: 09:3 Aug 08, 1999 Survey Co:USGS
Rotation:

Filename: ar85all.avg

Channels: Ch1 Ch2 Ch3 Ch4 Ch5 Ch3 Ch4 Plotted: 11:03 Dec 08, 2000

<EMI - ElectroMagnetic Instruments > 
whistler mtn.,nv

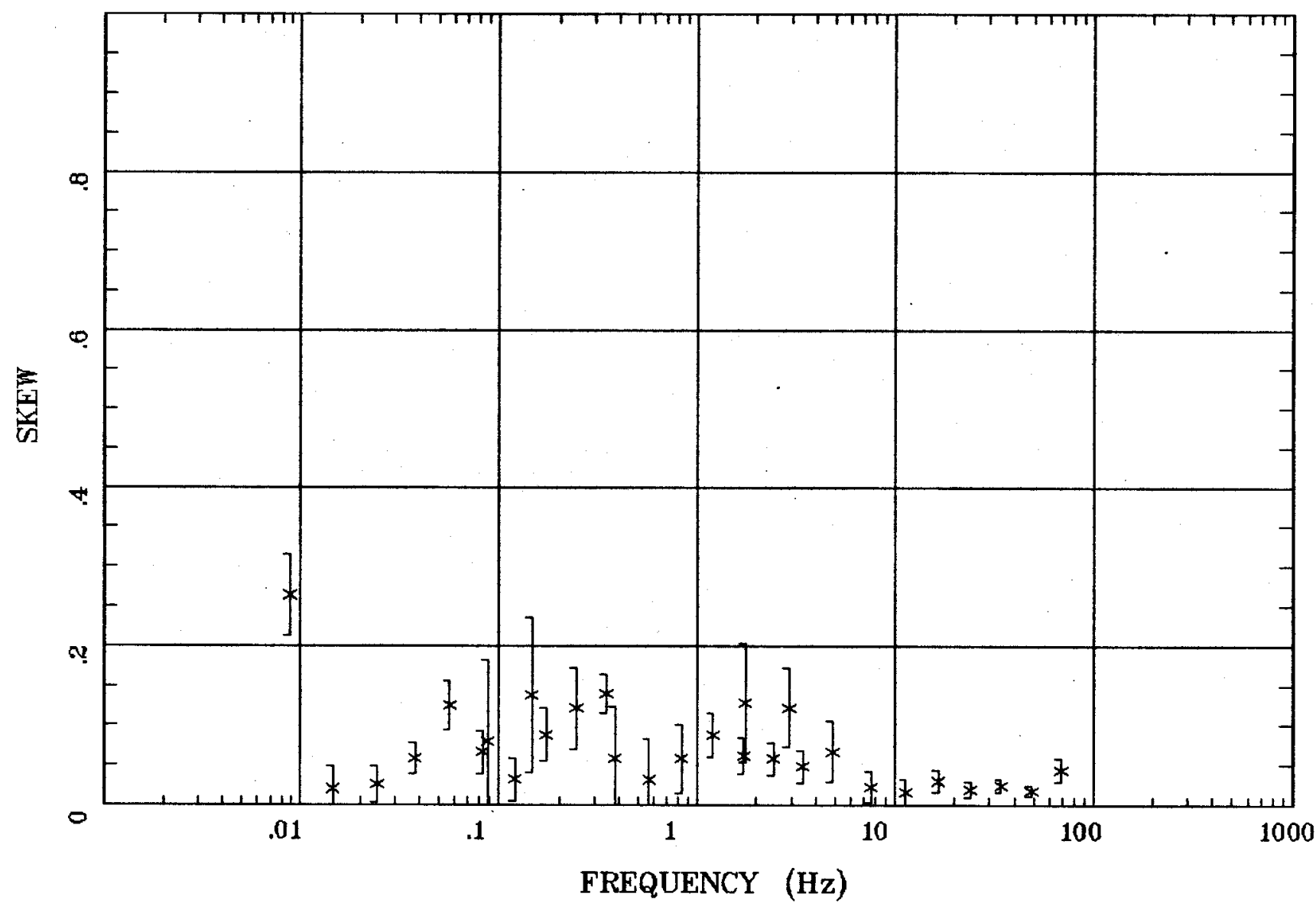

Client:

Remate: none

Acquired: 09:3 Aug 08, 1999

Survey Co:USGS
Rotation:

Filename: ar85all.avg

Channels: Ch1 Ch2 Ch3 Ch4 Ch5 Ch3 Ch4

Plotted: 11:03 Dec 08, 2000

$<$ EMI - ElectroMagnetic Instruments > 
E MULT Coh.

whistler mtn.,nv

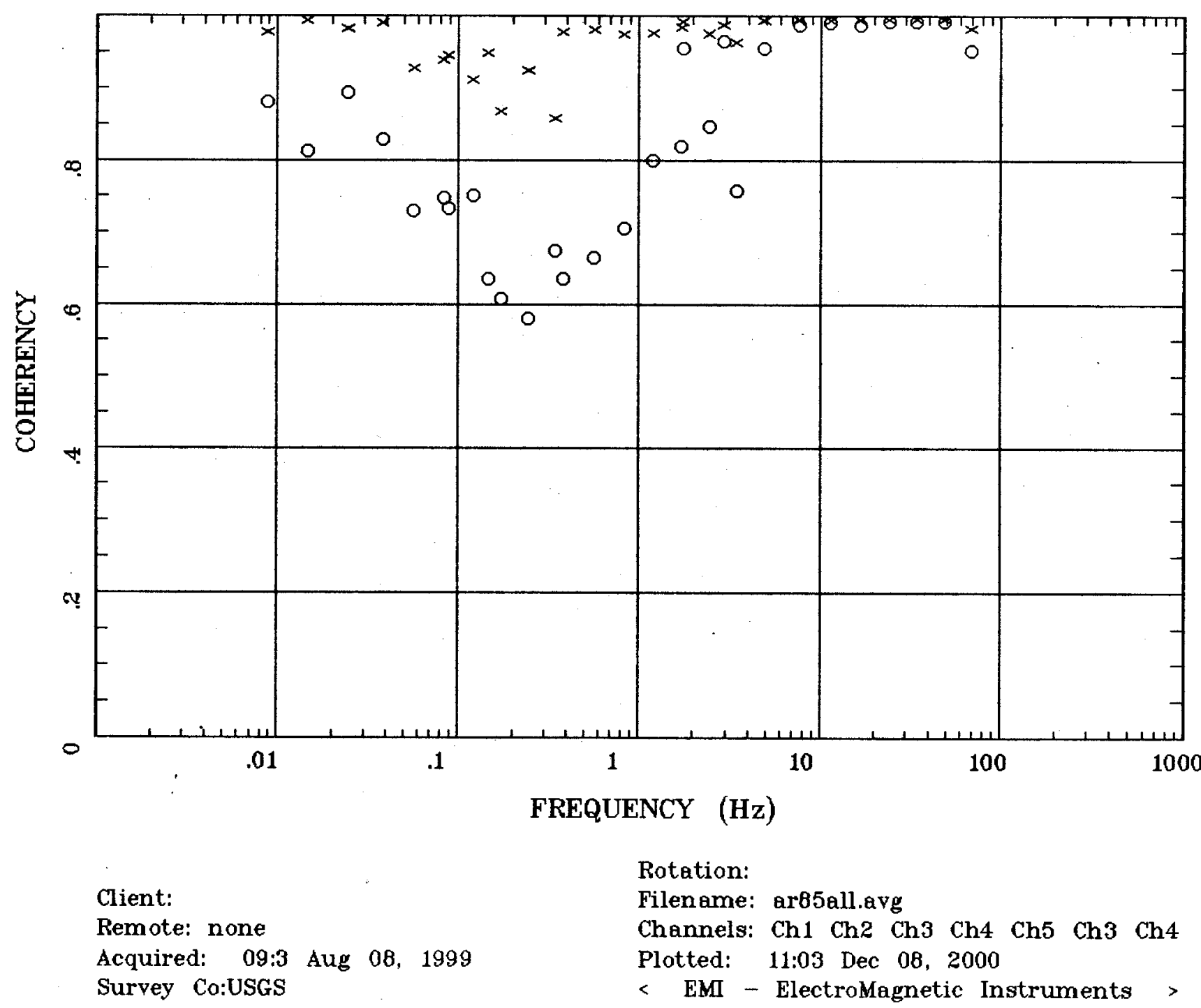




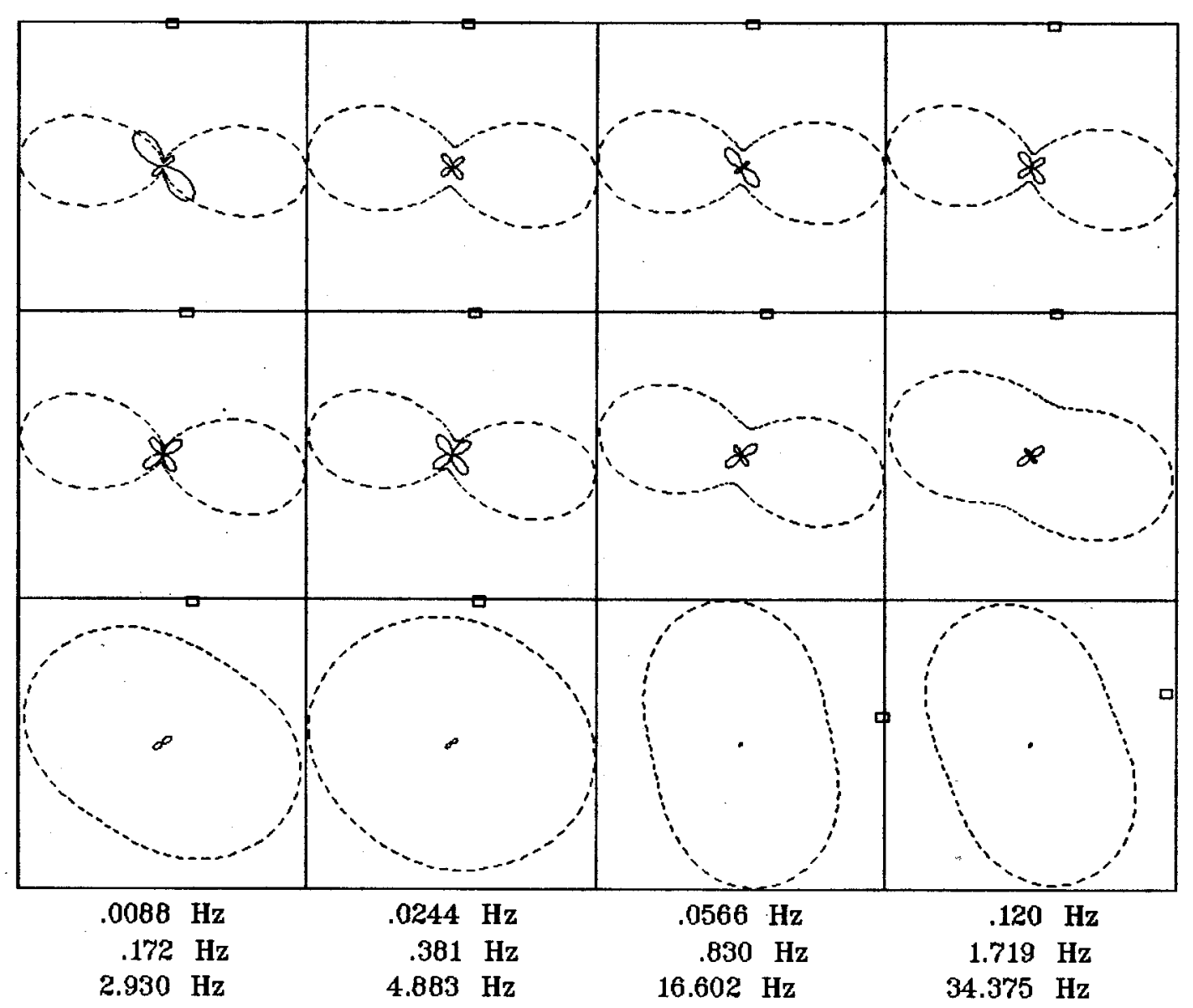

Client:

Remote: none

Acquired: 09:3 Aug 08, 1999 Survey Co:USGS
Rotation:

Filename: ar85all.avg

Channels: Ch1 Ch2 Ch3 Ch4 Ch5 Ch3 Ch4 Plotted: 11:03 Dec 08, 2000

< EMI - ElectroMagnetic Instruments 


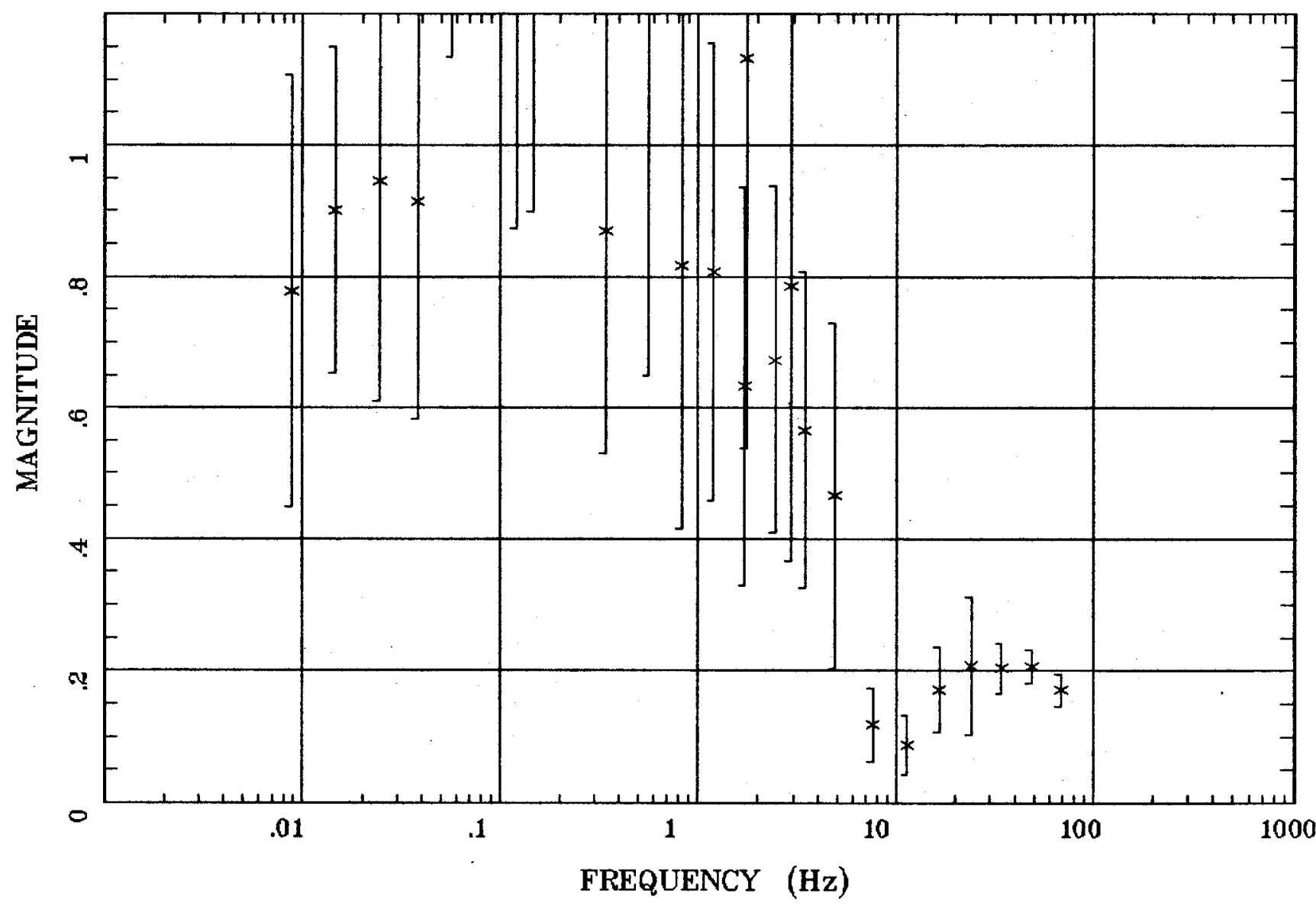

Client:

Remote: none

Acquired: 09:3 Aug 08, 1999 Survey Co:USGS
Rotation:

Filename: ar85all.avg

Channels: Ch1 Ch2 Ch3 Ch4 Ch5 Ch3 Ch4 Plotted: 11:03 Dec 08, 2000

$<$ EMI - ElectroMagnetic Instruments 


\section{Station 85}

TIPPER STRIKE

whistler mtn.,nv

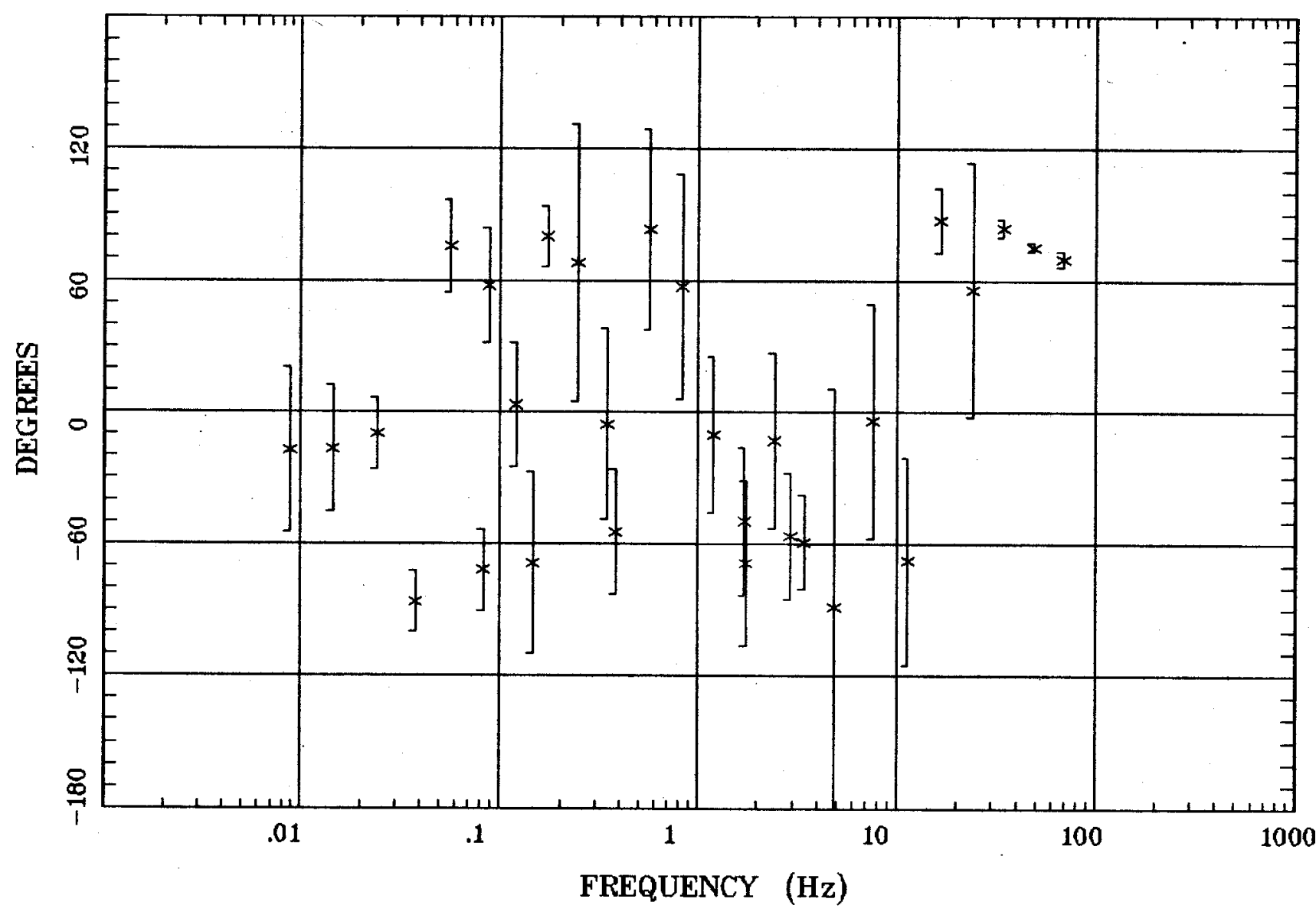

Client:

Remote: none

Acquired: 09:3 Aug 08, 1999

Survey Co:USGS
Rotation:

Filename: ar85all.avg

Channels: Ch1 Ch2 Ch3 Ch4 Ch5 Ch3 Ch4

Plotted: 11:03 Dec 08, 2000

< EMI - ElectroMagnetic Instruments > 
HzHx.x Coh HzHy.o

whistler mtn.,nv

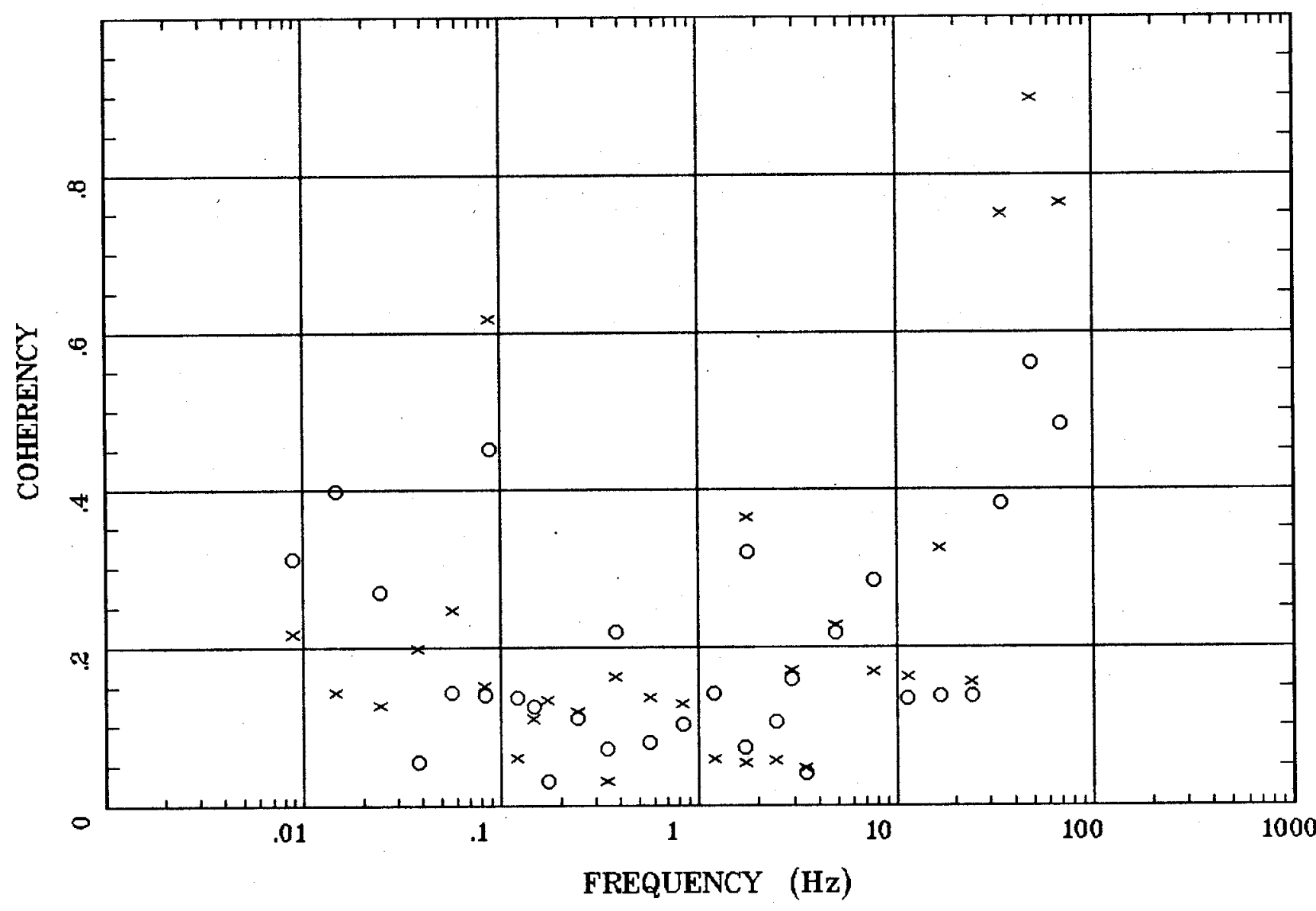

Client:

Remote: none

Acquired: 09:3 Aug 08, 1999 Survey Co:USGS
Rotation:

Filename: ar85all.avg

Channels: Ch1 Ch2 Ch3 Ch4 Ch5 Ch3 Ch4 Plotted: 11:03 Dec 08, 2000

< EMI - ElectroMagnetic Instruments 
APPARENT RESISTIVITY

Diamond Valley, NV

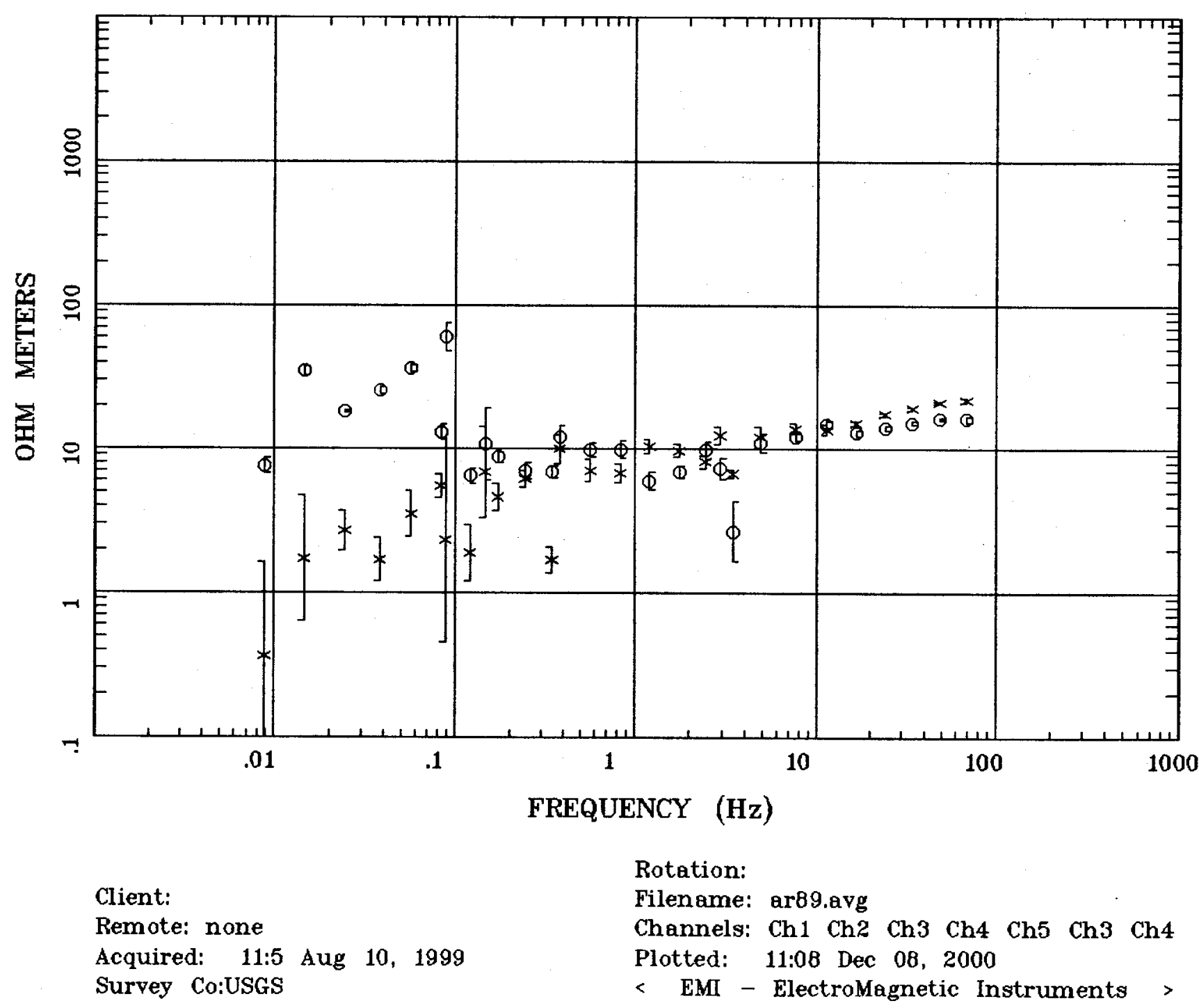


IMPEDANCE PHASE

Diamond Valley, NV

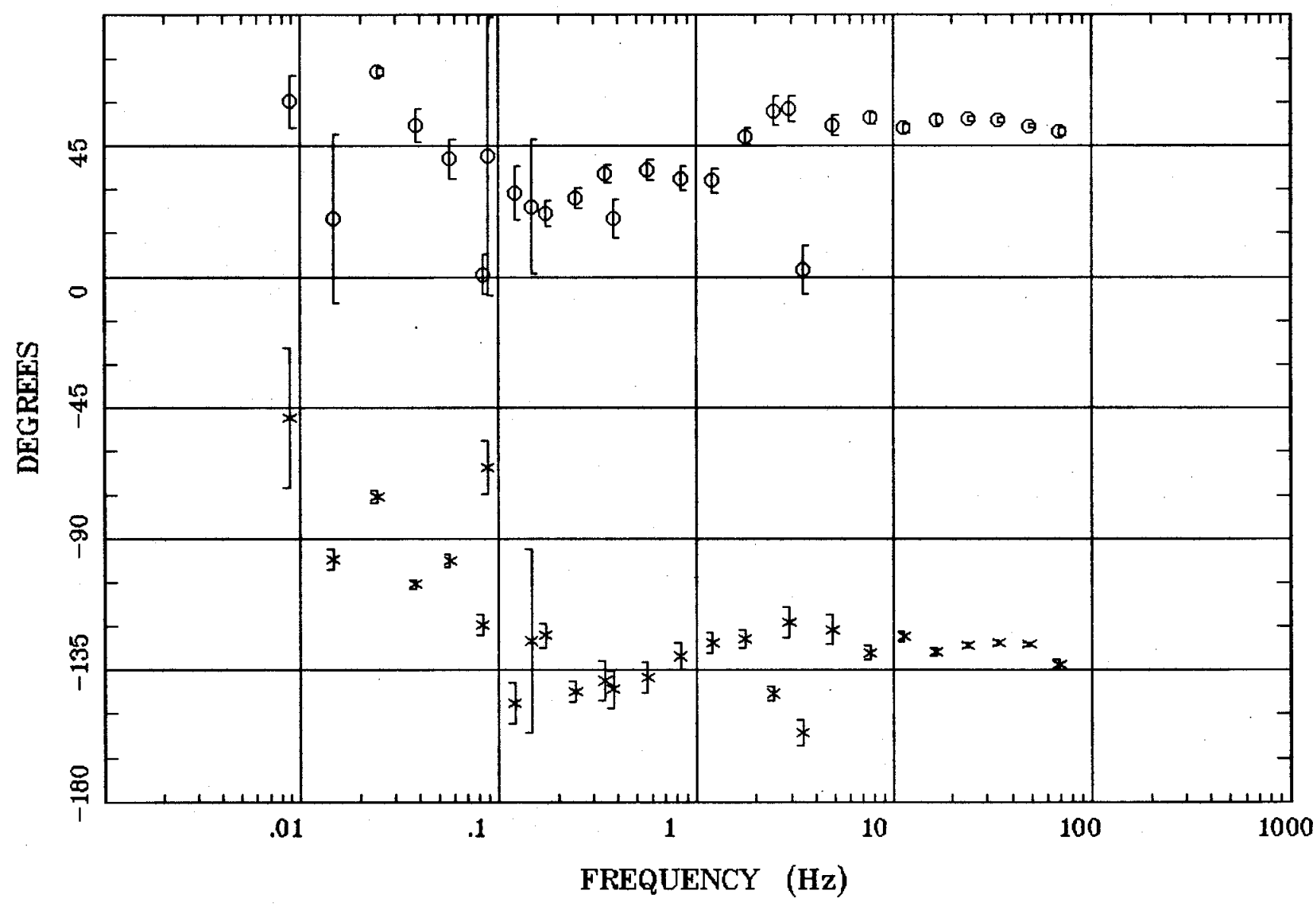

Client:

Remote: none

Acquired: 11:5 Aug 10, 1999 Survey Co:USGS
Rotation:

Filename: ar89.avg

Channels: Ch1 Ch2 Ch3 Ch4 Ch5 Ch3 Ch4 Plotted: 11:08 Dec 08, 2000

< EMI - ElectroMagnetic Instruments 
Diamond Valley, NV

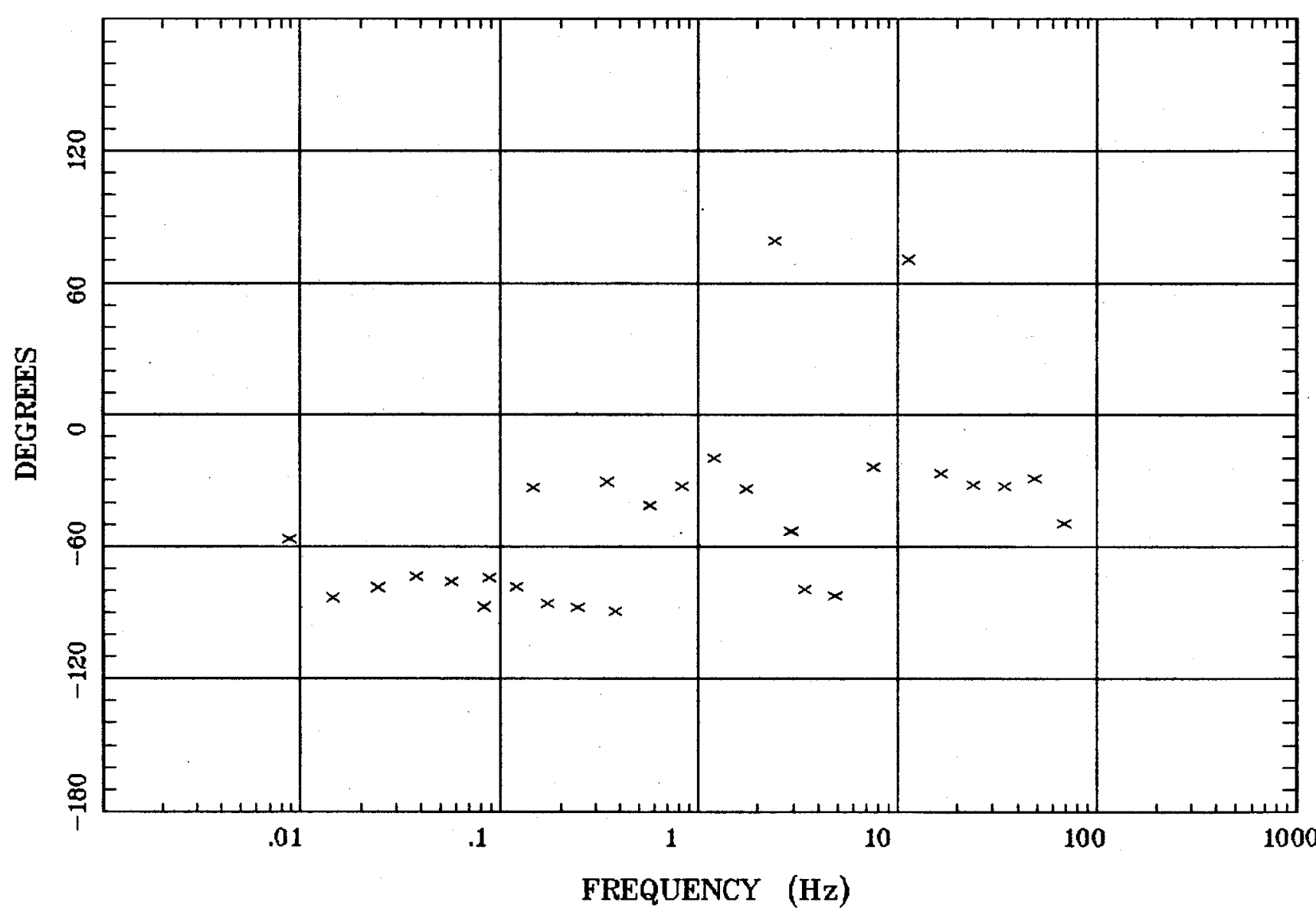

Client:

Remote: none

Acquired: 11:5 Aug 10, 1999 Survey Co:USGS
Rotation:

Filename: ar89.avg

Channels: Ch1 Ch2 Ch3 Ch4 Ch5 Ch3 Ch4 Plotted: 11:08 Dec 08, 2000

$<$ EMI - ElectroMagnetic Instruments 
IMPEDANCE SKEW

Diamond Valley, NV

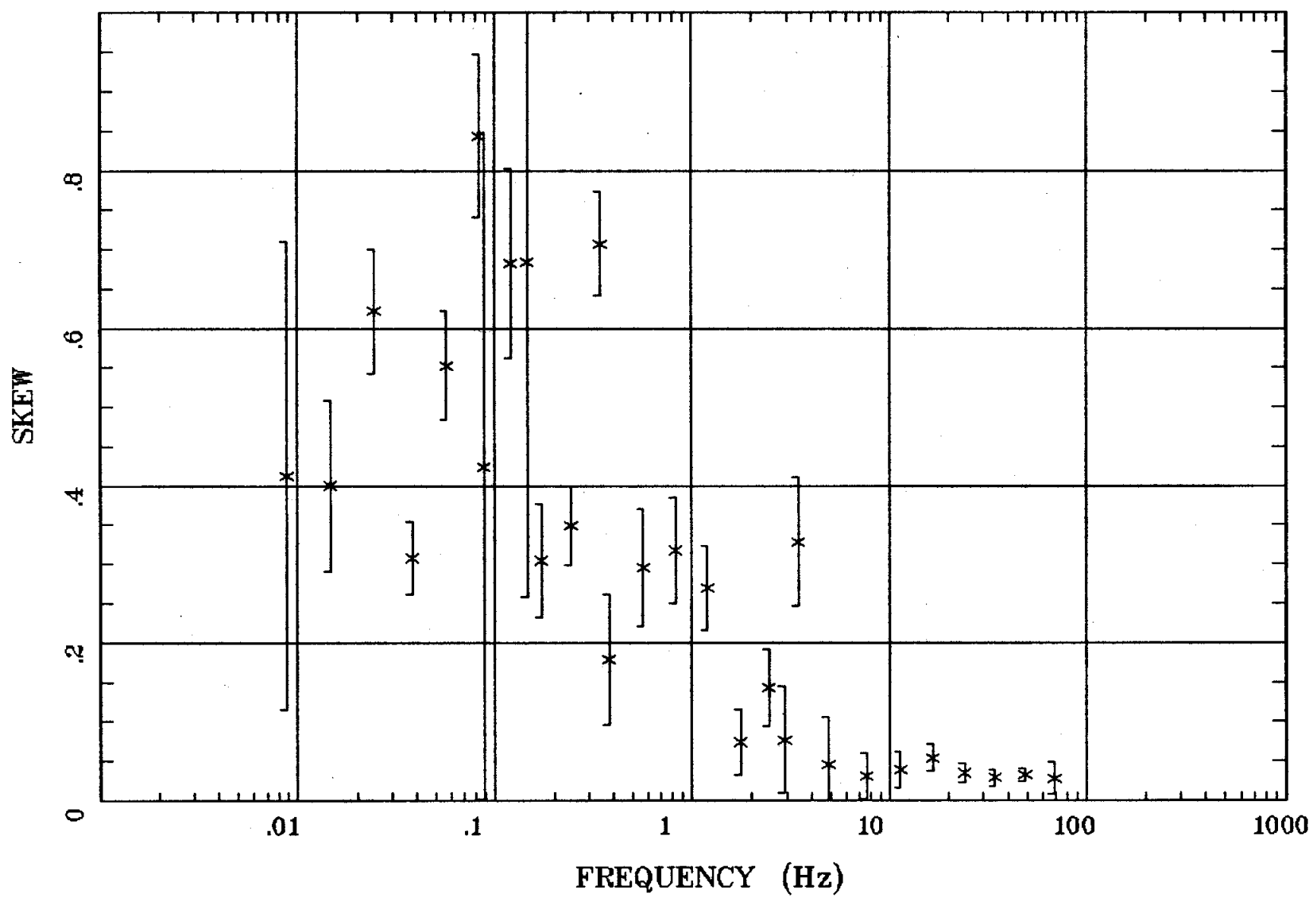

Client:

Remote: none

Acquired: 11:5 Aug 10, 1999 Survey Co:USGS
Rotation:

Filename: ar89.avg

Channels: Ch1 Ch2 Ch3 Ch4 Ch5 Ch3 Ch4 Plotted: 11:08 Dec 08, 2000

$<$ EMI - ElectroMagnetic Instruments > 


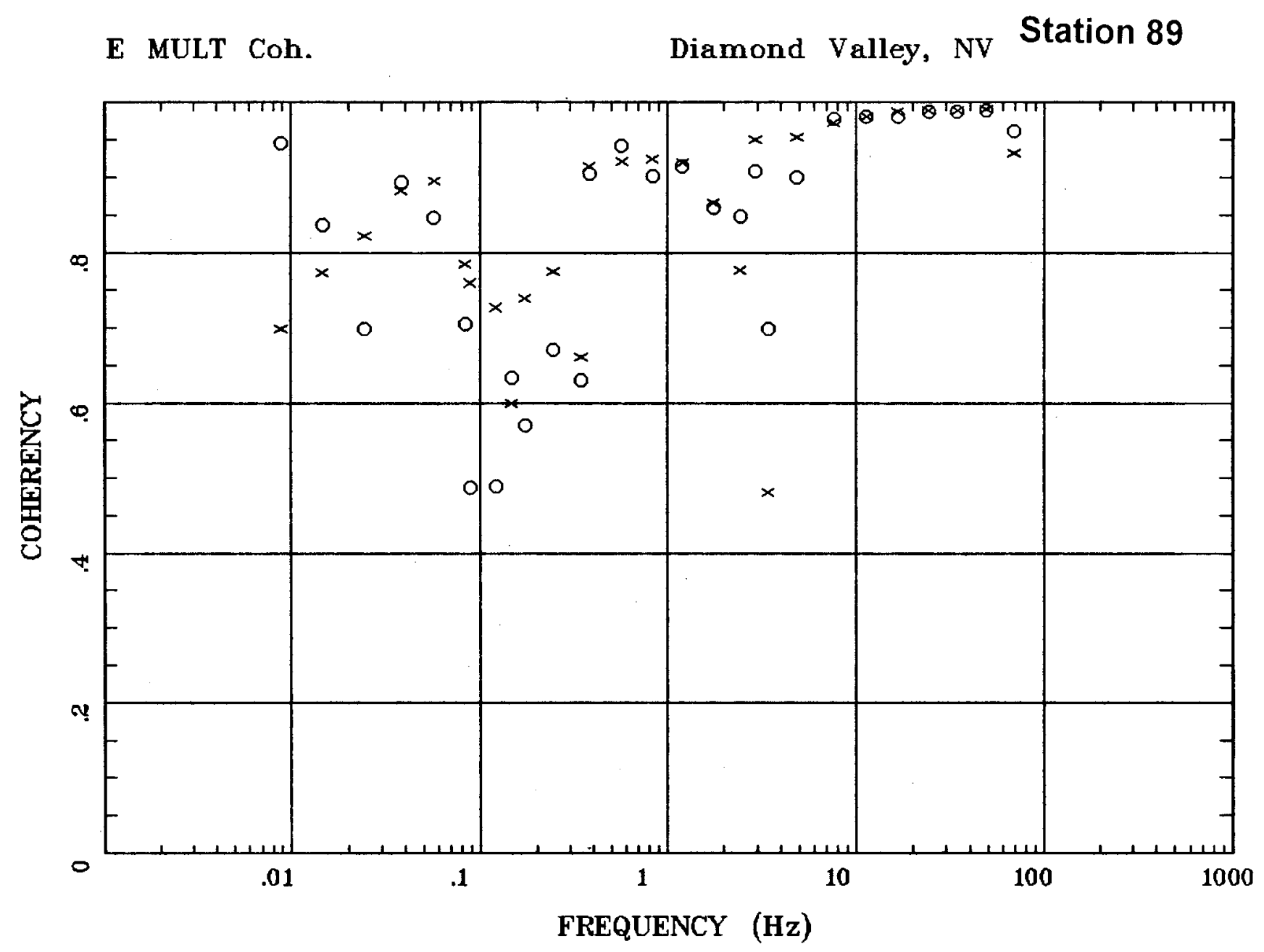

Client:

Remote: none

Acquired: 11:5 Aug 10, 1999

Survey Co:USGS
Rotation:

Filename: ar89.avg

Channels: Ch1 Ch2 Ch3 Ch4 Ch5 Ch3 Ch4

Plotted: 11:08 Dec 08, 2000

< EMI - ElectroMagnetic Instruments > 


\section{Station 89}

TIPPER MAGNITUDE

Diamond Valley, NV

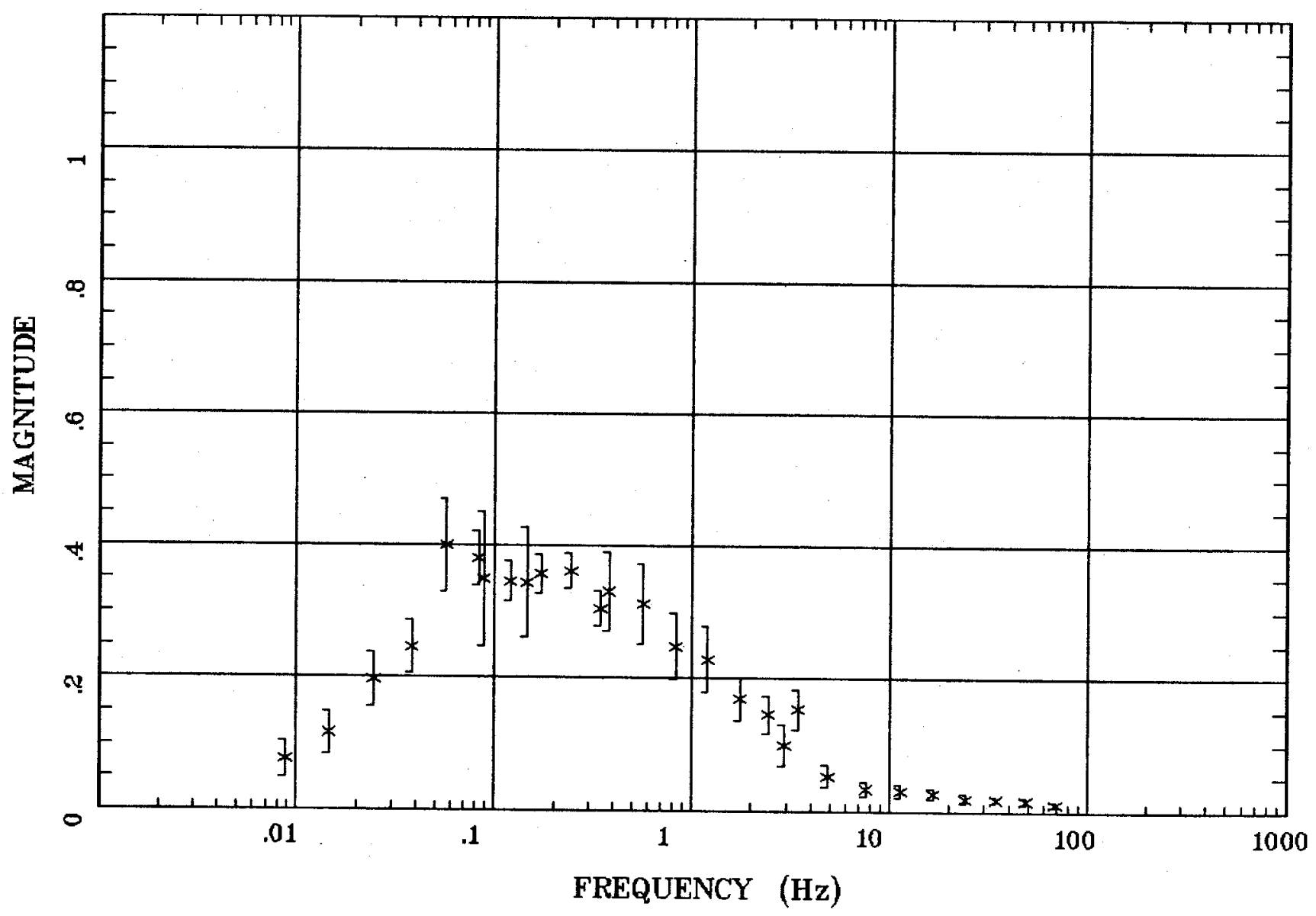

Client:

Rotation:

Filename: ar89.avg

Remote: none

Acquired: 11:5 Aug 10, 1999

Survey Co:USGS

Channels: Ch1 Ch2 Ch3 Ch4 Ch5 Ch3 Ch4

Plotted: 11:08 Dec 08, 2000

$<$ EMI - ElectroMagnetic Instruments > 
TIPPER STRIKE

Diamond Valley, NV

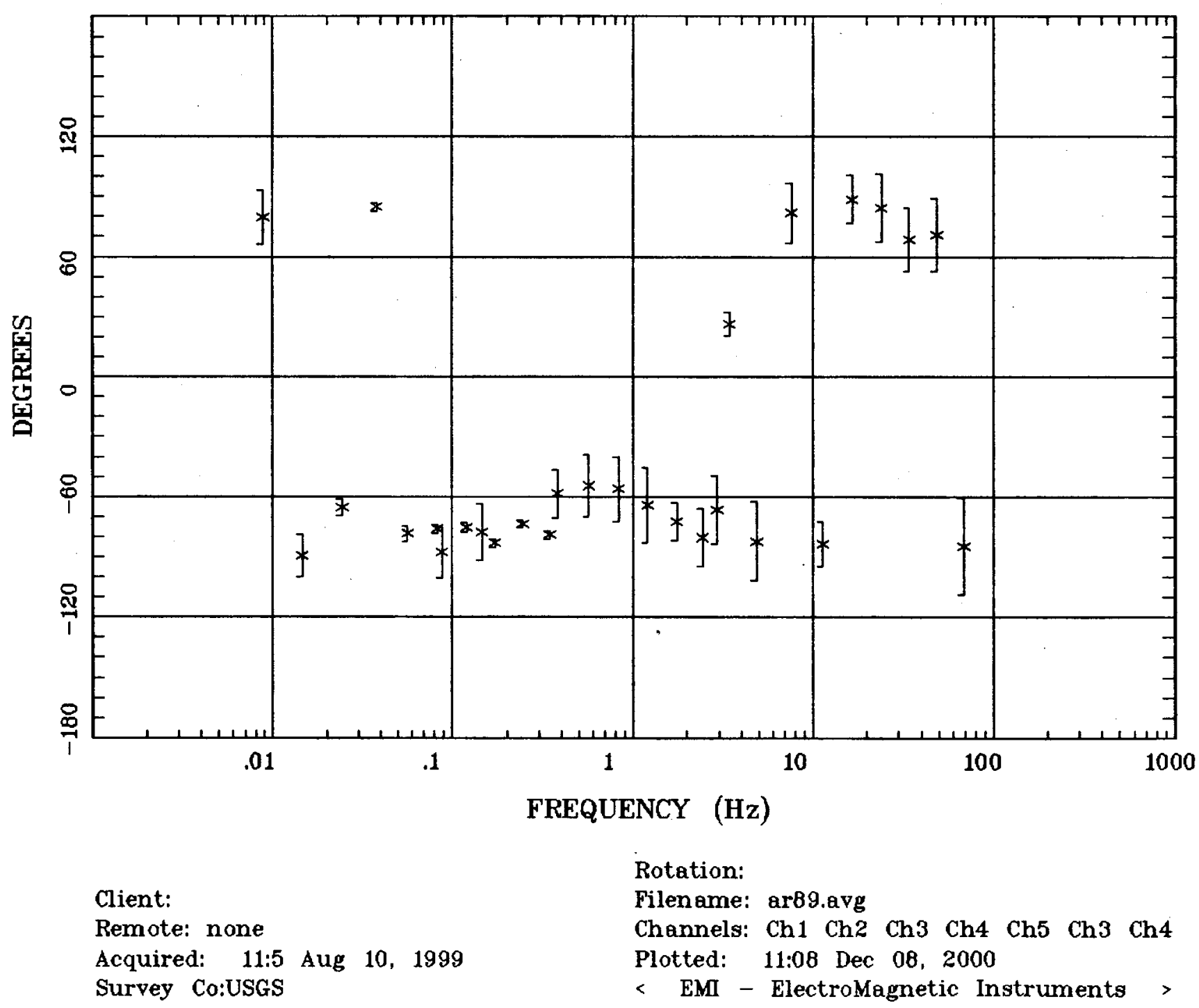




\section{Station 89}

HzHx.x Coh HzHy.o

Diamond Valley, NV

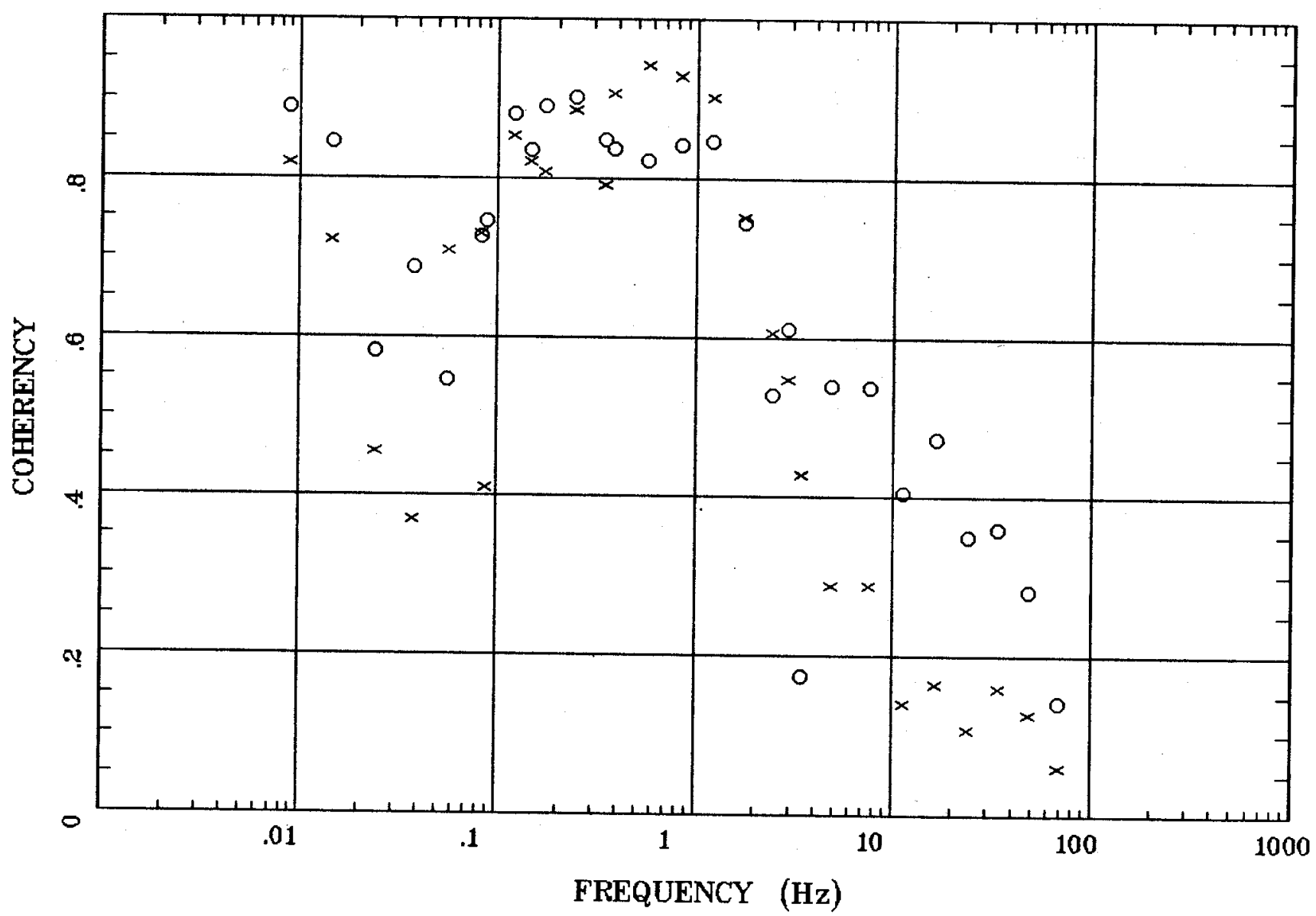

Client:

Remote: none

Acquired: 11:5 Aug 10, 1999 Survey Co:USGS
Rotation:

Filename: ar89.avg

Channels: Ch1 Ch2 Ch3 Ch4 Ch5 Ch3 Ch4

Plotted: 11:08 Dec 08, 2000

< EMI - ElectroMagnetic Instruments > 


\section{APPARENT RESISTIVITY}

Newark Lake, NV 100K

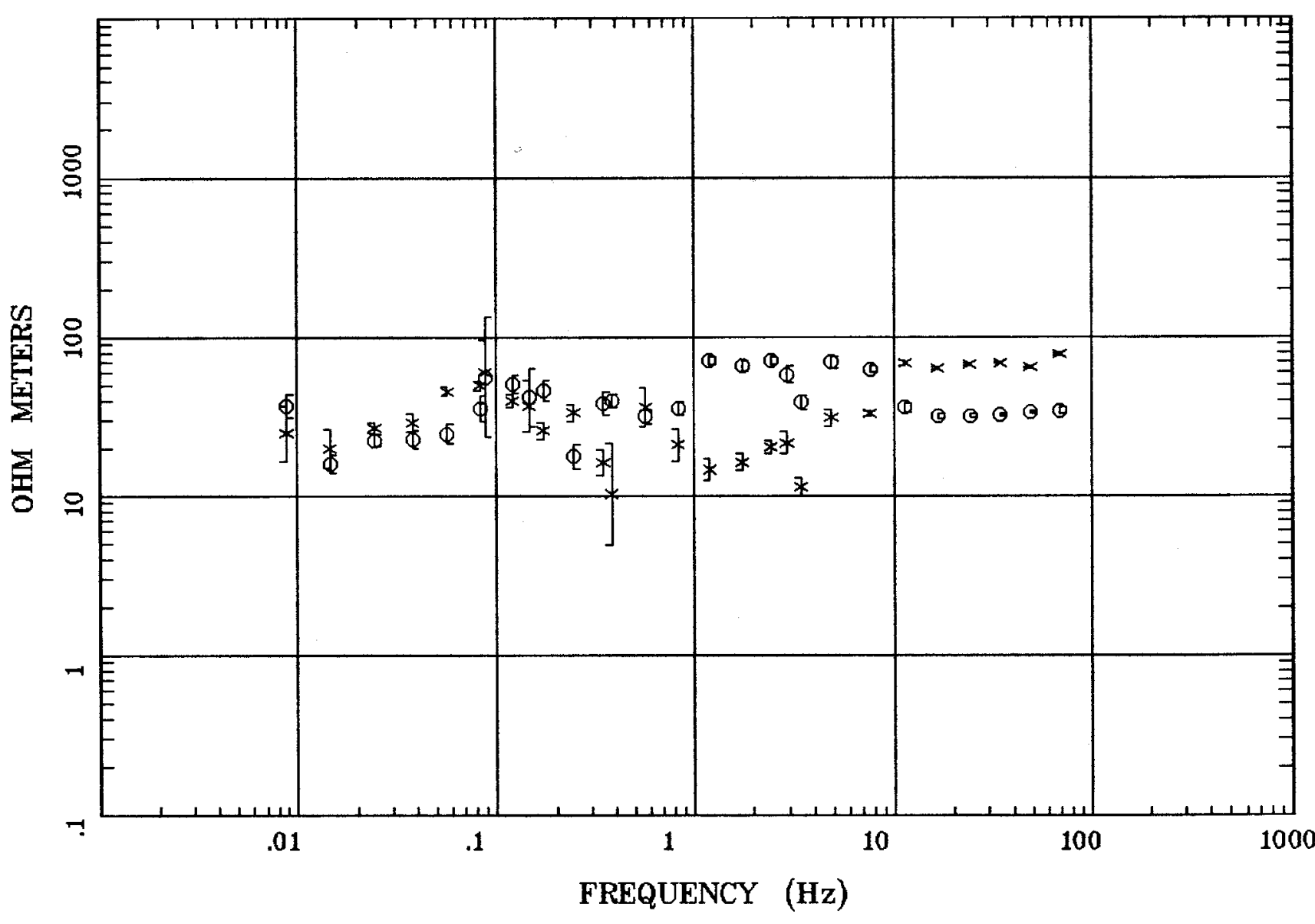

Client:

Remote: none

Acquired: 09:2 Aug 08, 2000 Survey Co:USGS
Rotation:

Filename: ar109b.avg

Channels: Ch1 Ch2 Ch3 Ch4 Ch5 Ch3 Ch4 Plotted: 10:53 Dec 08, 2000

$<$ EMI - ElectroMagnetic Instruments 
Station 109

IMPEDANCE PHASE

Newark Lake, NV 100K

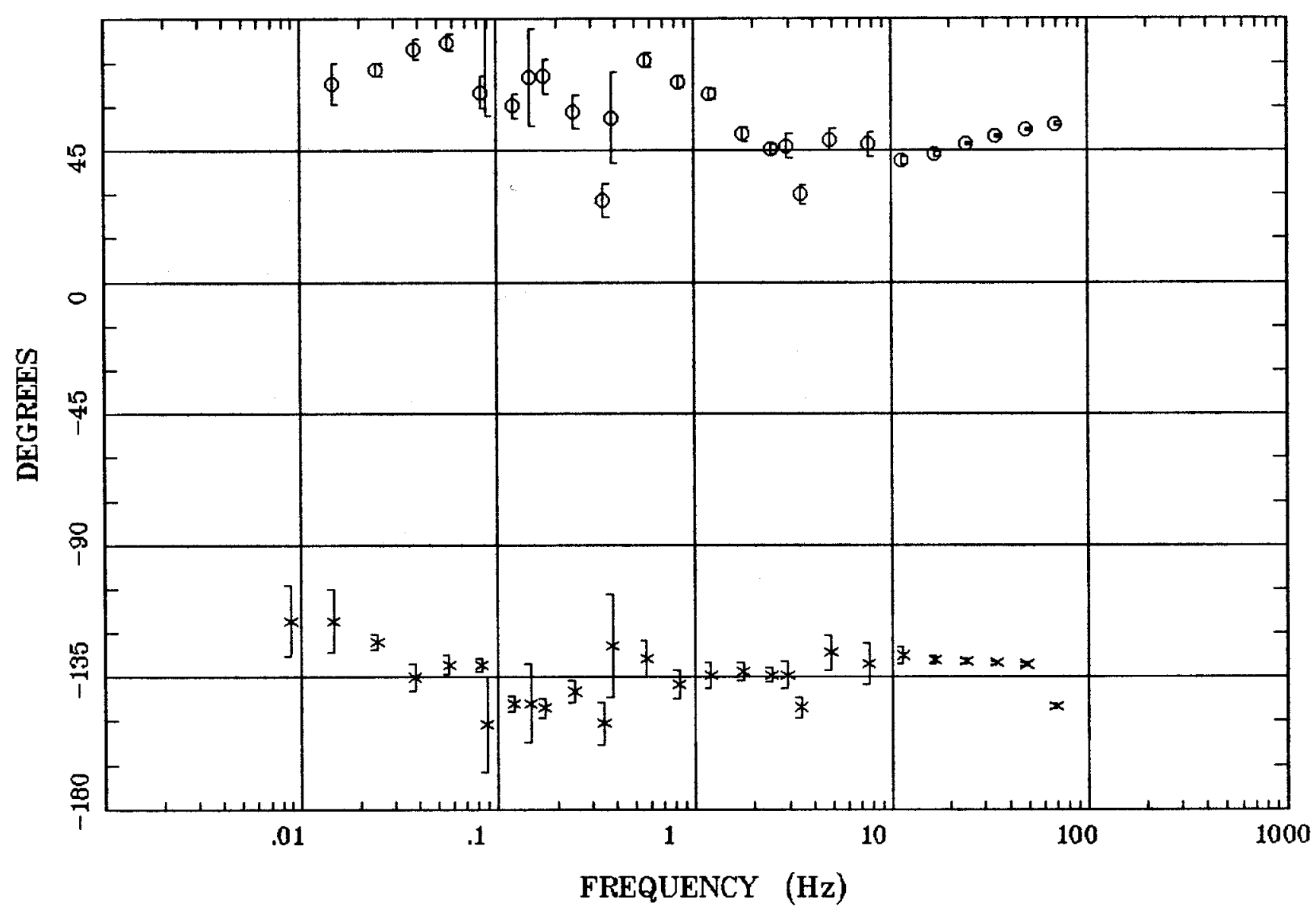

Client:

Remote: none

Rotation:

Filename: ar109b.avg

Channels: Ch1 Ch2 Ch3 Ch4 Ch5 Ch3 Ch4

Plotted: 10:53 Dec 08, 2000

Acquired: 09:2 Aug 08, 2000

< EMI - ElectroMagnetic Instruments > 


\section{Station 109}

ROTATION ANGLE

Newark Lake, NV 100K

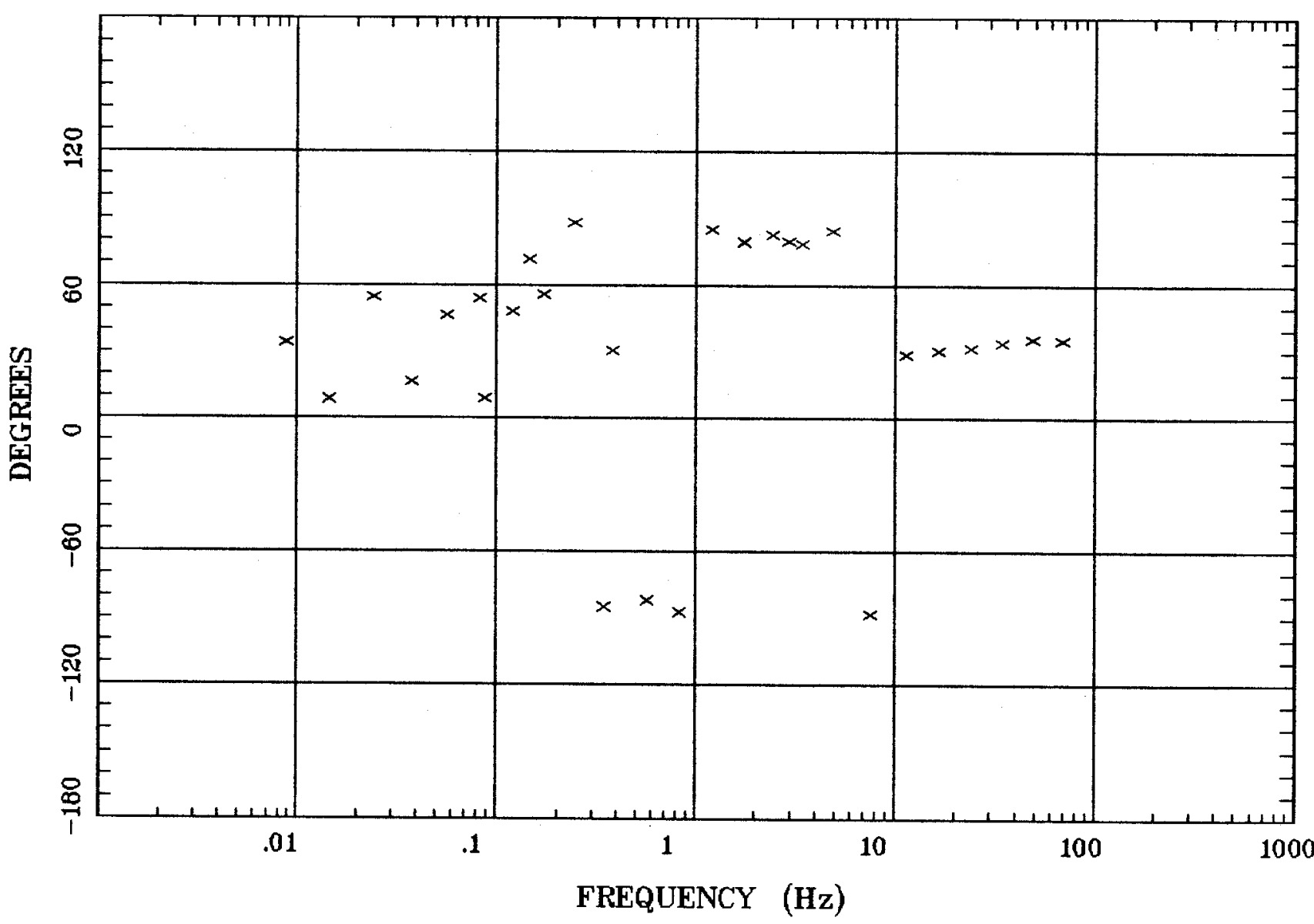

Client:

Remote: none

Acquired: 09:2 Aug 08, 2000 Survey Co:USGS
Rotation:

Filename: ar109b.avg

Channels: Ch1 Ch2 Ch3 Ch4 Ch5 Ch3 Ch4

Plotted: 10:53 Dec 08, 2000

< EMI - ElectroMagnetic Instruments 


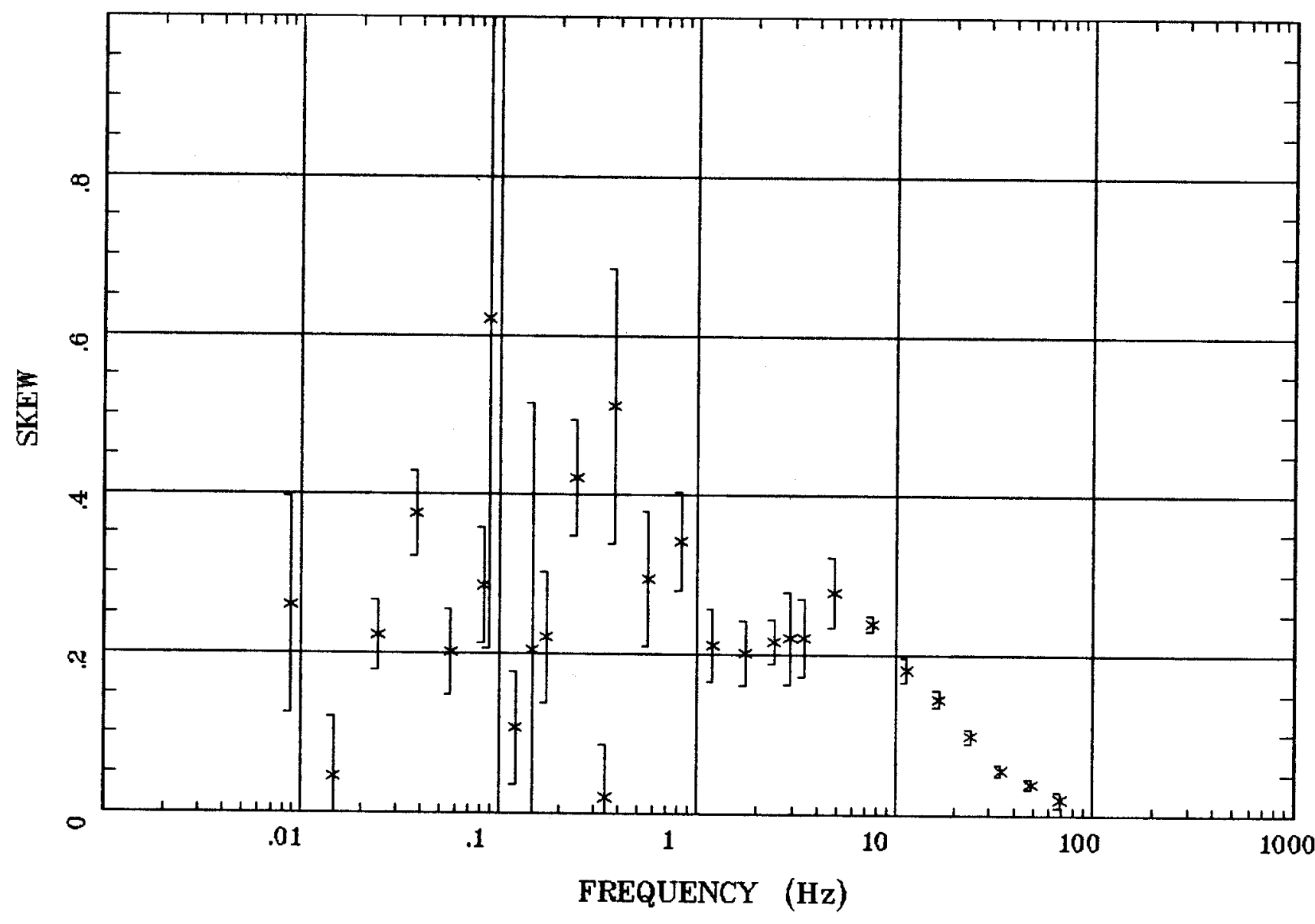

Client:

Remote: none

Acquired: 09:2 Aug 08, 2000 Survey Co:USGS
Rotation:

Filename: ar109b.avg

Channels: Ch1 Ch2 Ch3 Ch4 Ch5 Ch3 Ch4 Plotted: 10:53 Dec 08, 2000

$<$ EMI - ElectroMagnetic Instruments 
E MULT Coh.

Newark Lake, NV 100K

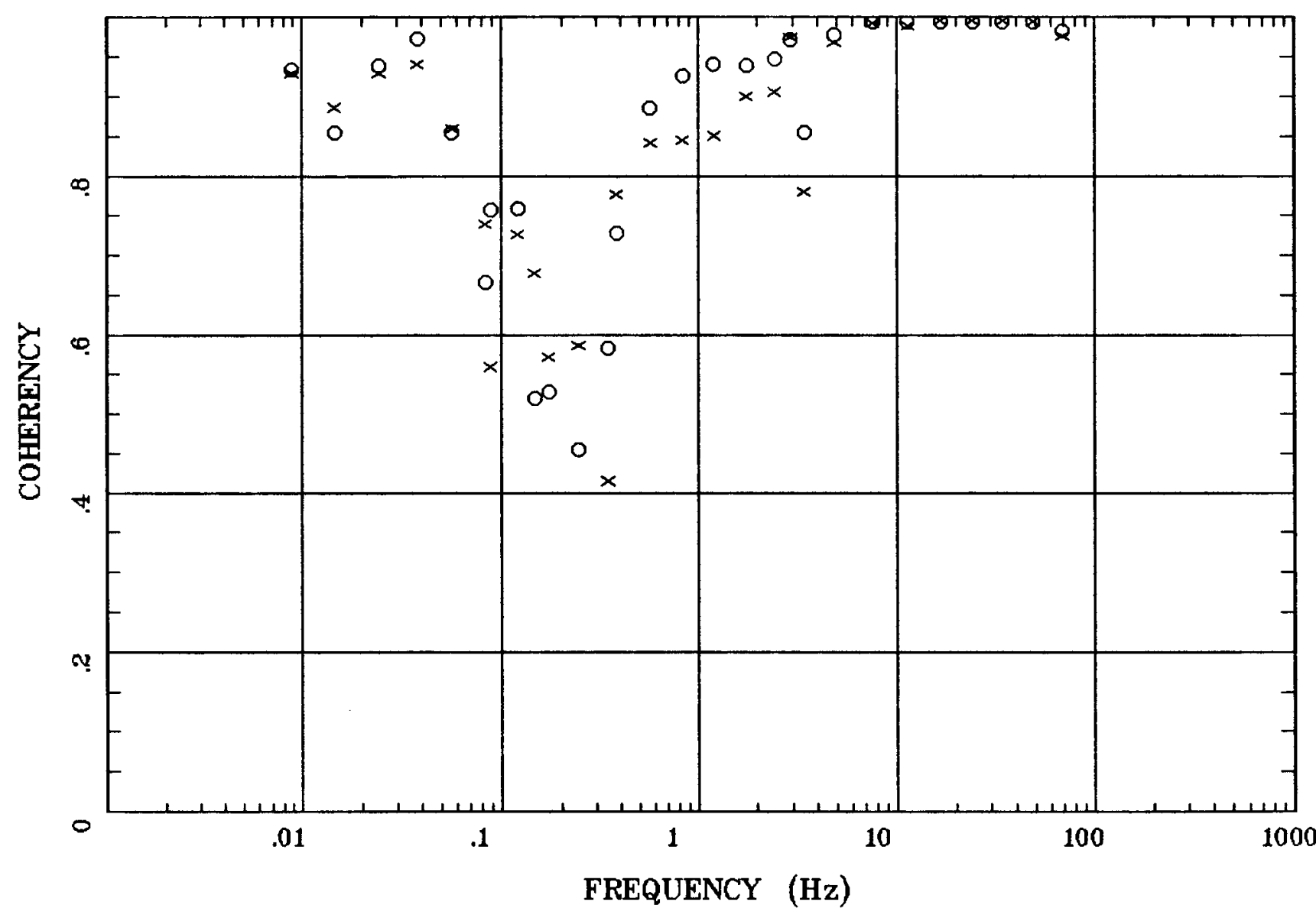

Client:

Remote: none

Acquired: 09:2 Aug 08, 2000

Survey Co:USGS

Rotation:

Filename: ar109b.avg

Channels: Ch1 Ch2 Ch3 Ch4 Ch5 Ch3 Ch4 Plotted: 10:53 Dec 08, 2000

< EMI - ElectroMagnetic Instruments 


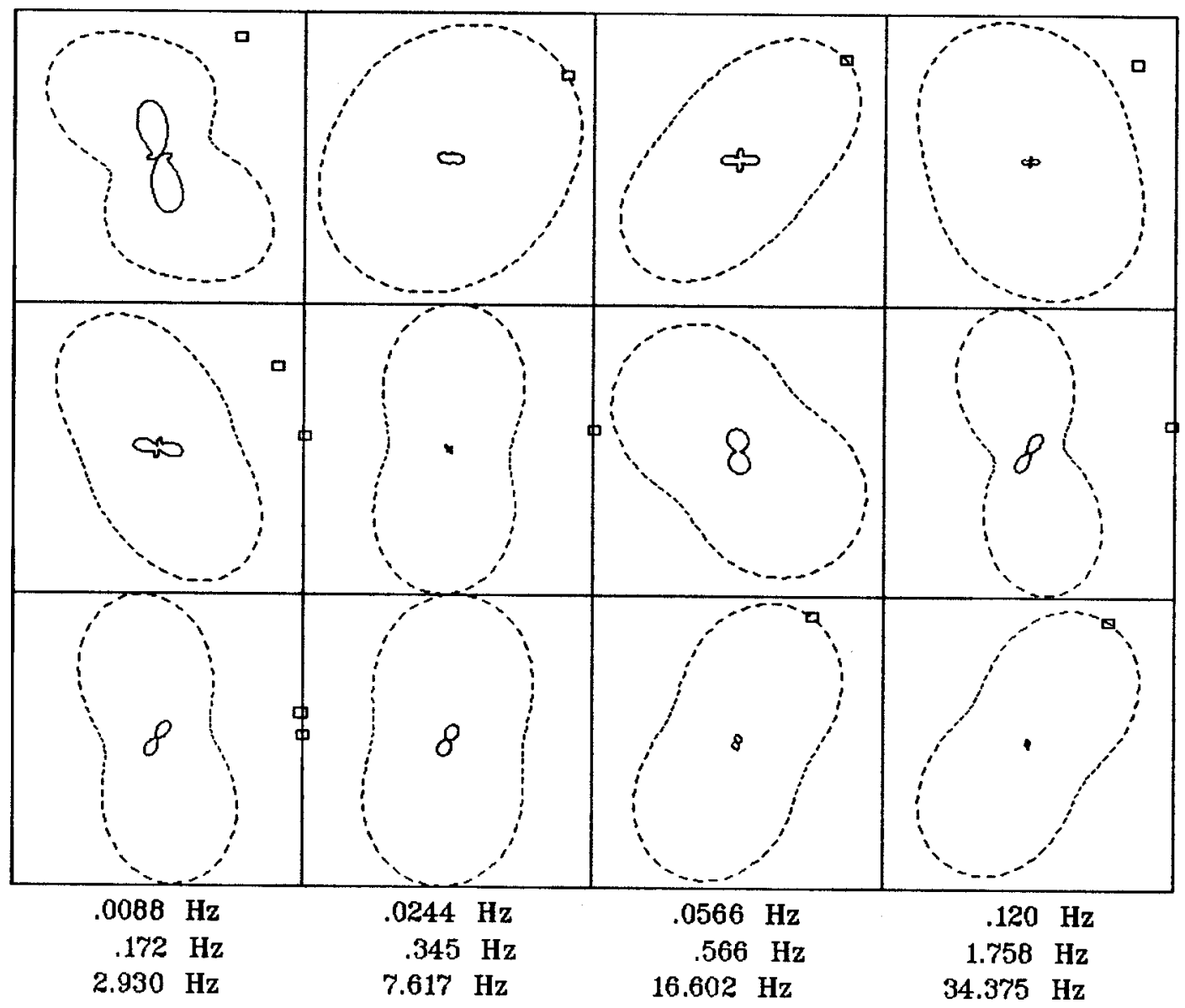

Client:

Remote: none

Acquired: 09:2 Aug 08, 2000 Survey Co:USGS
Rotation:

Filename: ar109b.avg

Channels: Ch1 Ch2 Ch3 Ch4 Ch5 Ch3 Ch4 Plotted: 10:53 Dec 08, 2000

$<$ EMI - ElectroMagnetic Instruments > 


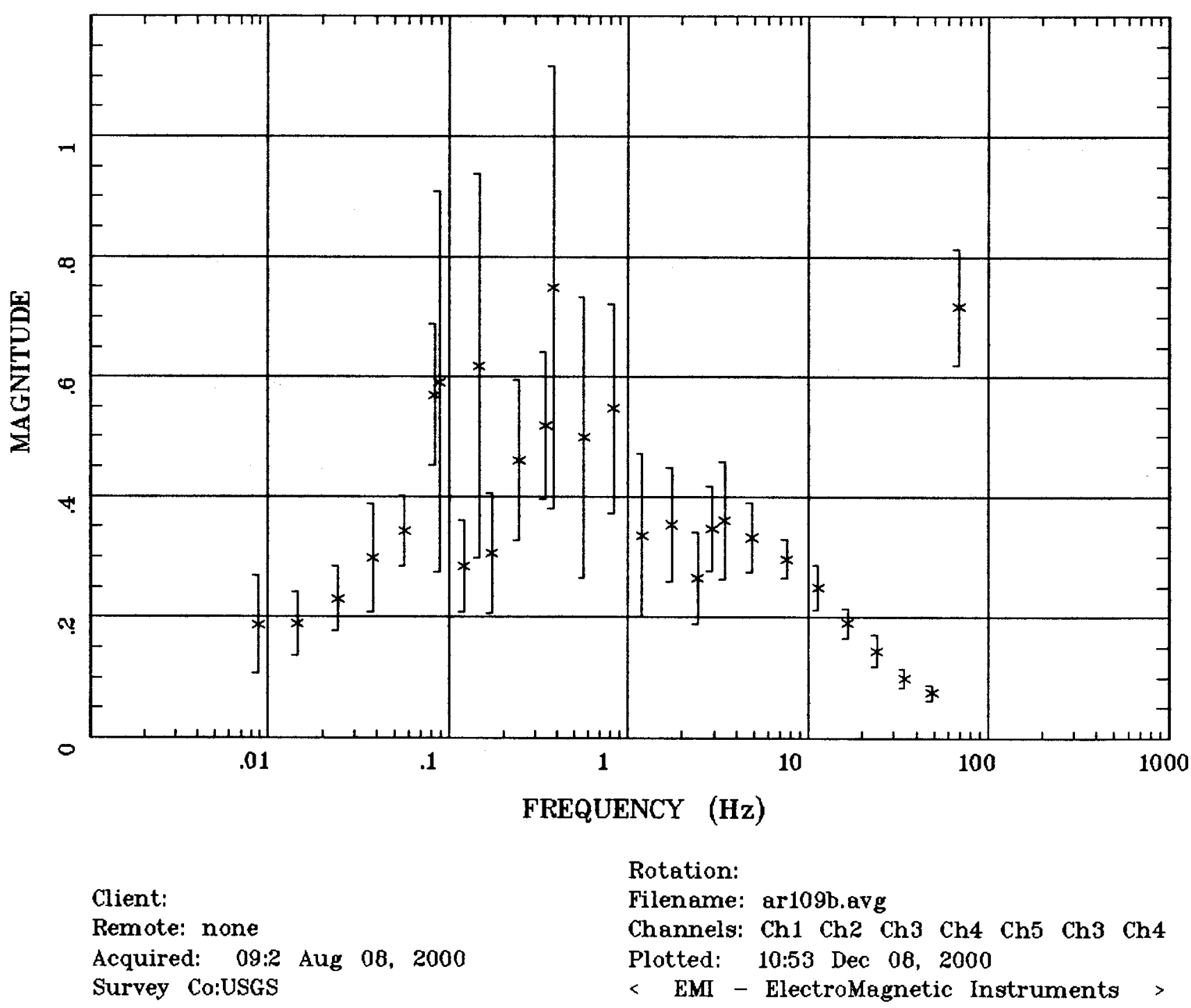


TIPPER STRIKE

Newark Lake, NV 100K

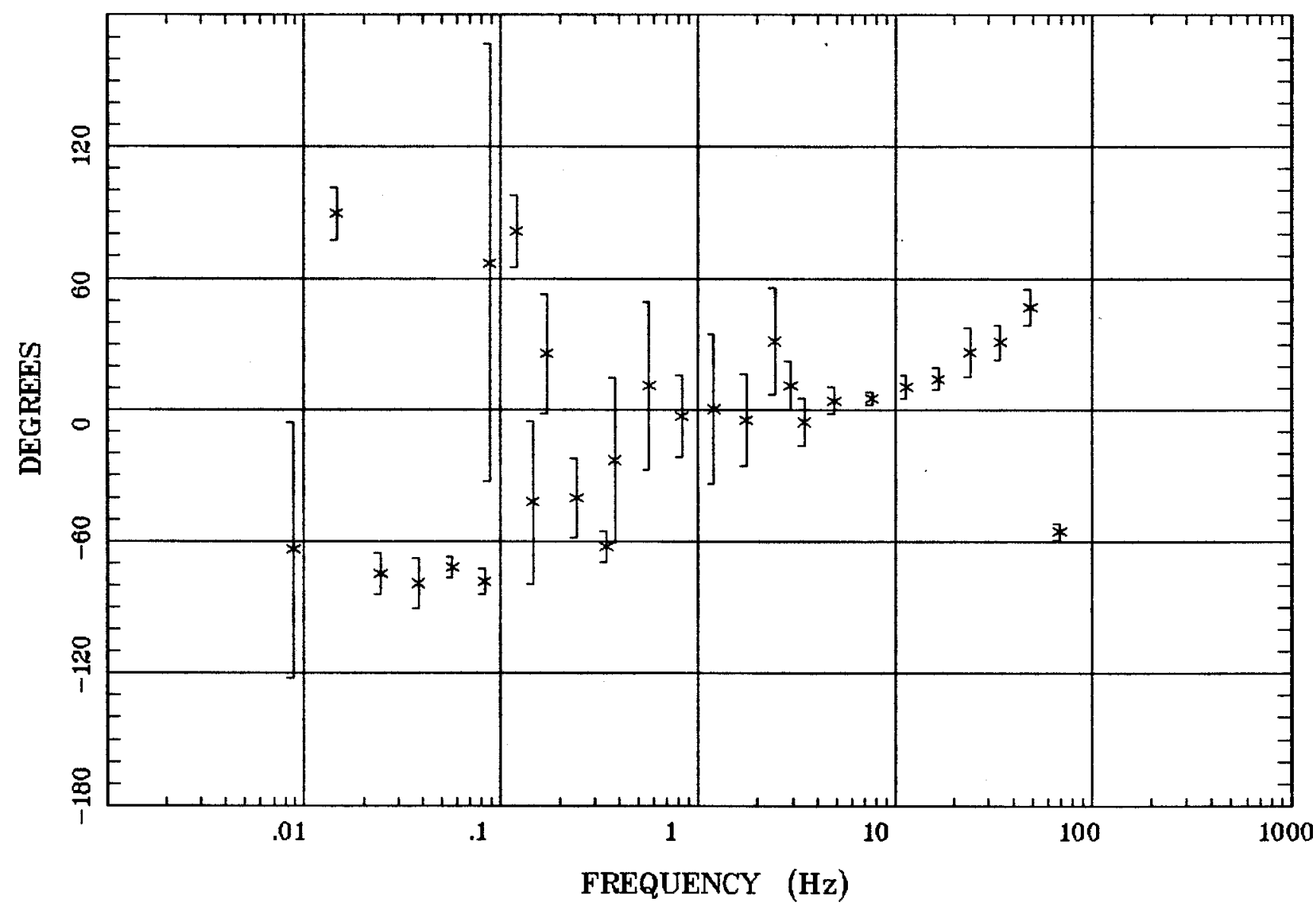

Client:

Remote: none

Acquired: 09:2 Aug 08, 2000

Survey Co:USGS
Rotation:

Filename: ar109b.avg

Channels: Ch1 Ch2 Ch3 Ch4 Ch5 Ch3 Ch4 Plotted: 10:53 Dec 08, 2000

< EMI - ElectroMagnetic Instruments > 


\section{Station 109}

\section{HzHx.x Coh HzHy.o}

Newark Lake, NV 100K

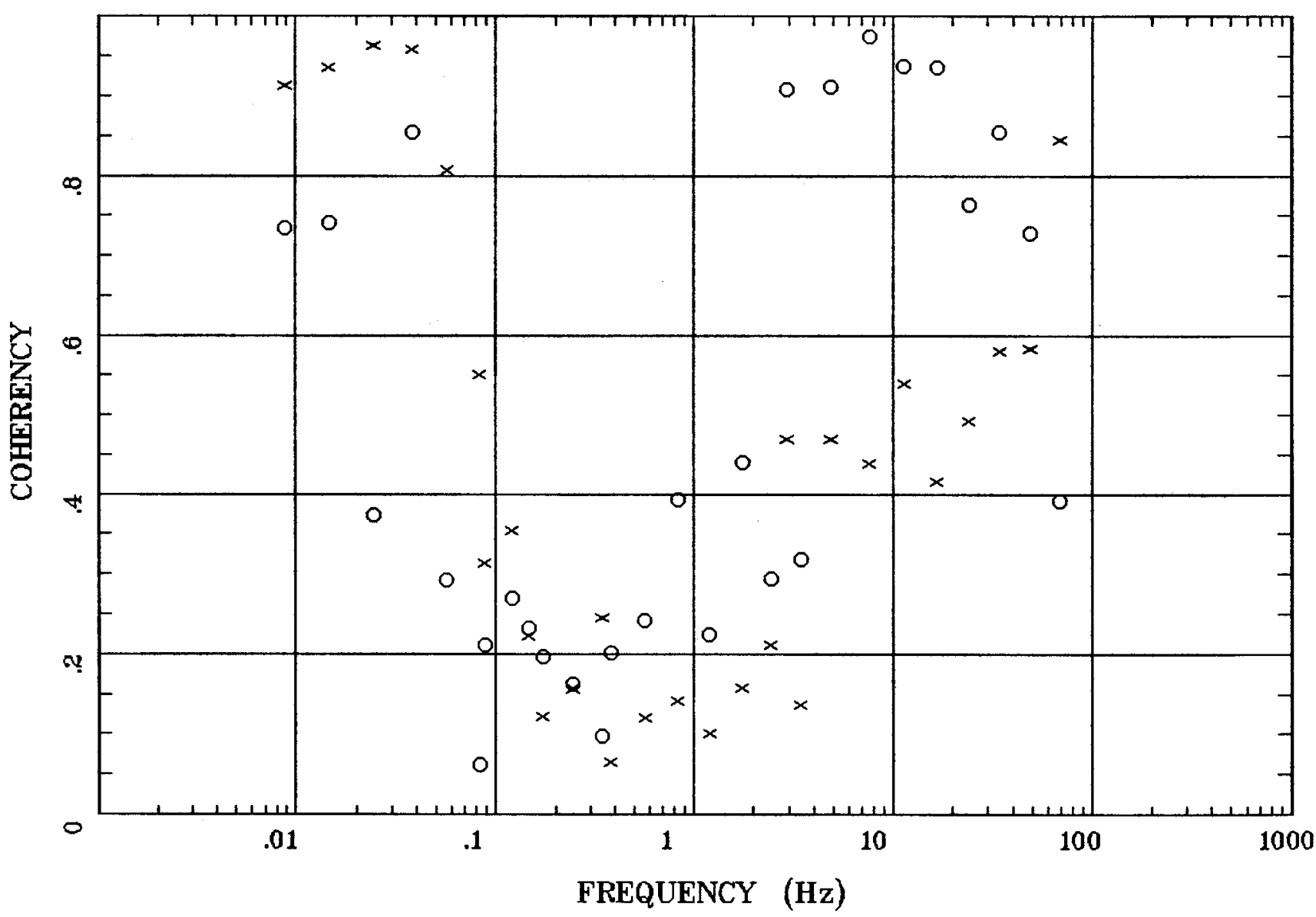

Client:

Remote: none

Acquired: 09:2 Aug 08, 2000 Survey Co:USGS
Rotation:

Filename: ar109b.avg

Channels: Ch1 Ch2 Ch3 Ch4 Ch5 Ch3 Ch4 Plotted: 10:53 Dec 08, 2000

< EMI - ElectroMagnetic Instruments 
Diamond Mtns., NV

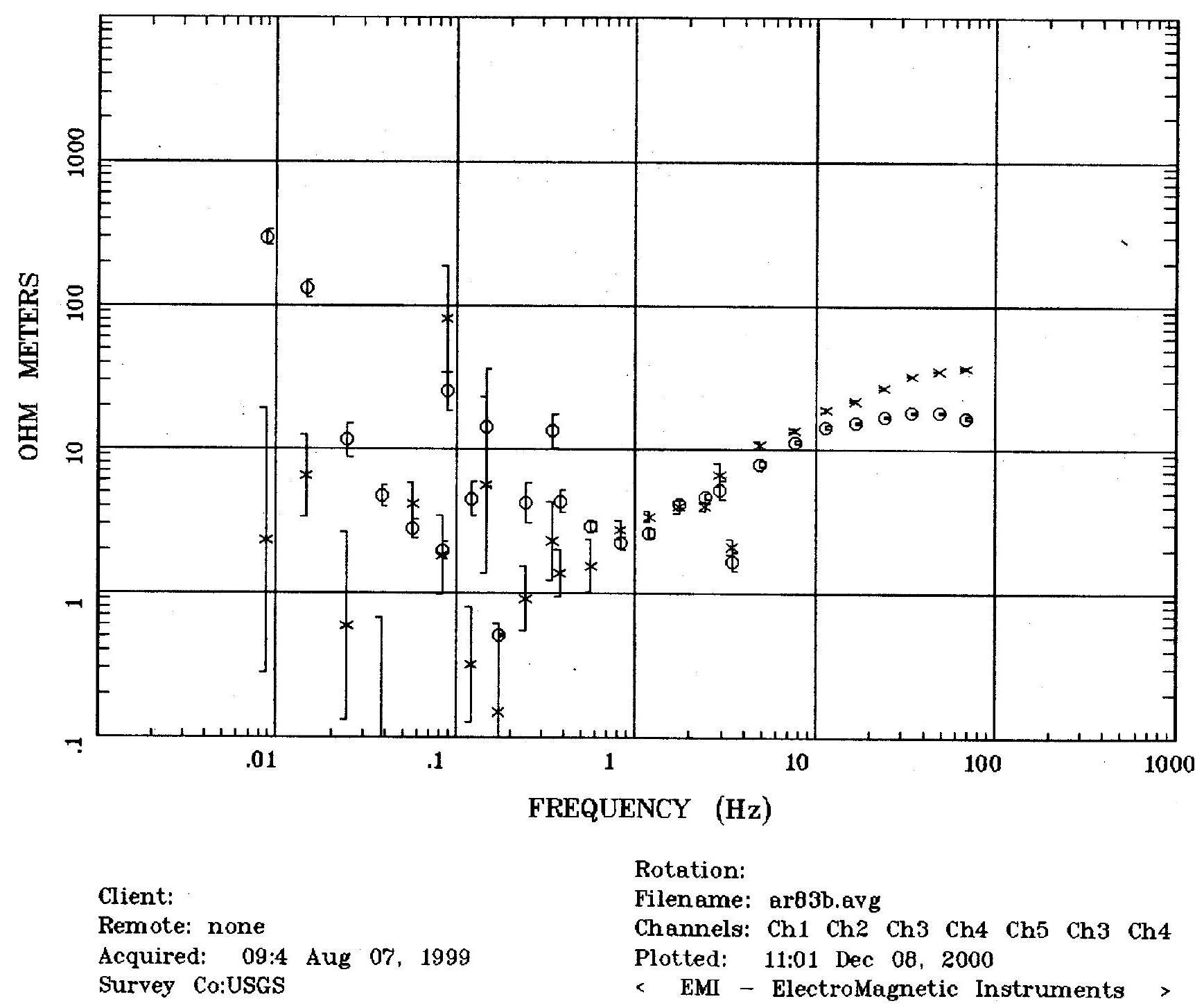


Diamond Mtns., NV

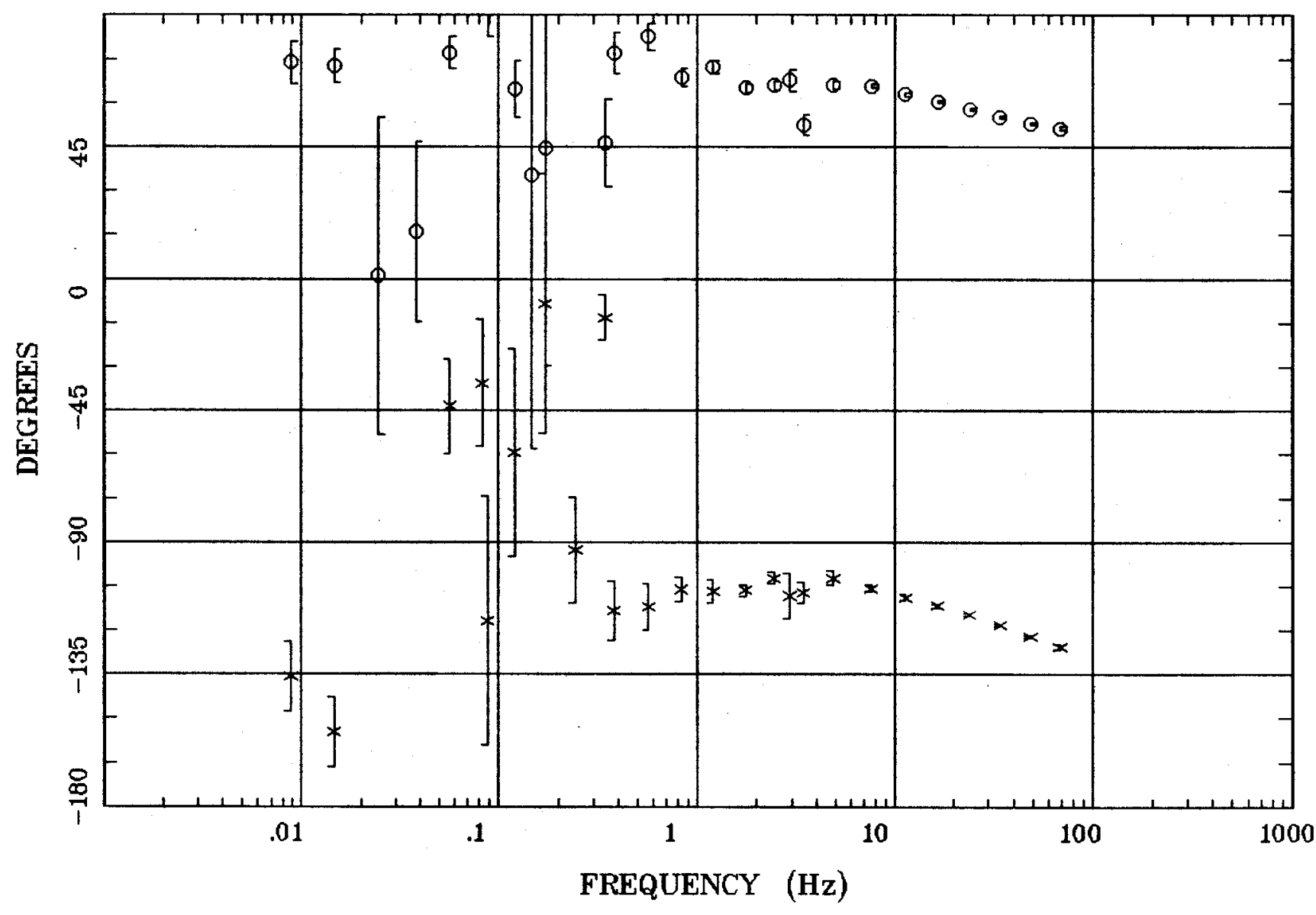

Client:

Remote: none

Acquired: 09:4 Aug 07, 1999

Survey Co:USGS
Rotation:

Filename: ar83b.avg

Channels: Ch1 Ch2 Ch3 Ch4 Ch5 Ch3 Ch4 Plotted: 11:01 Dec 08, 2000

$<$ EMI - ElectroMagnetic Instruments > 


\section{ROTATION ANGLE}

Diamond Mtns., NV

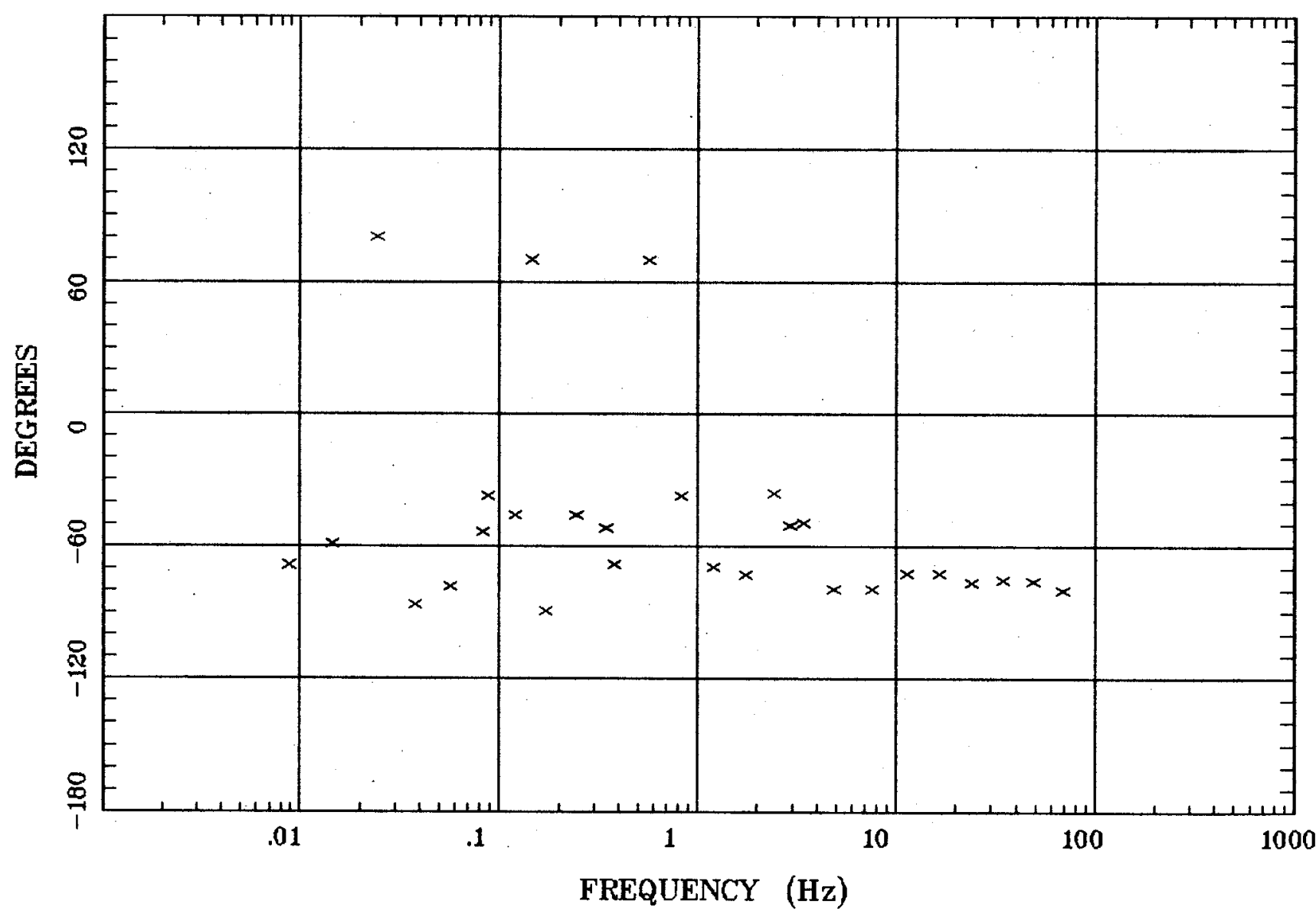

Client:

Remote: none

Acquired: 09:4 Aug 07, 1999

Survey Co:USGS
Rotation:

Filename: ar83b.avg

Channels: Ch1 Ch2 Ch3 Ch4 Ch5 Ch3 Ch4 Plotted: 11:01 Dec 08, 2000

< EMI - ElectroMagnetic Instruments 


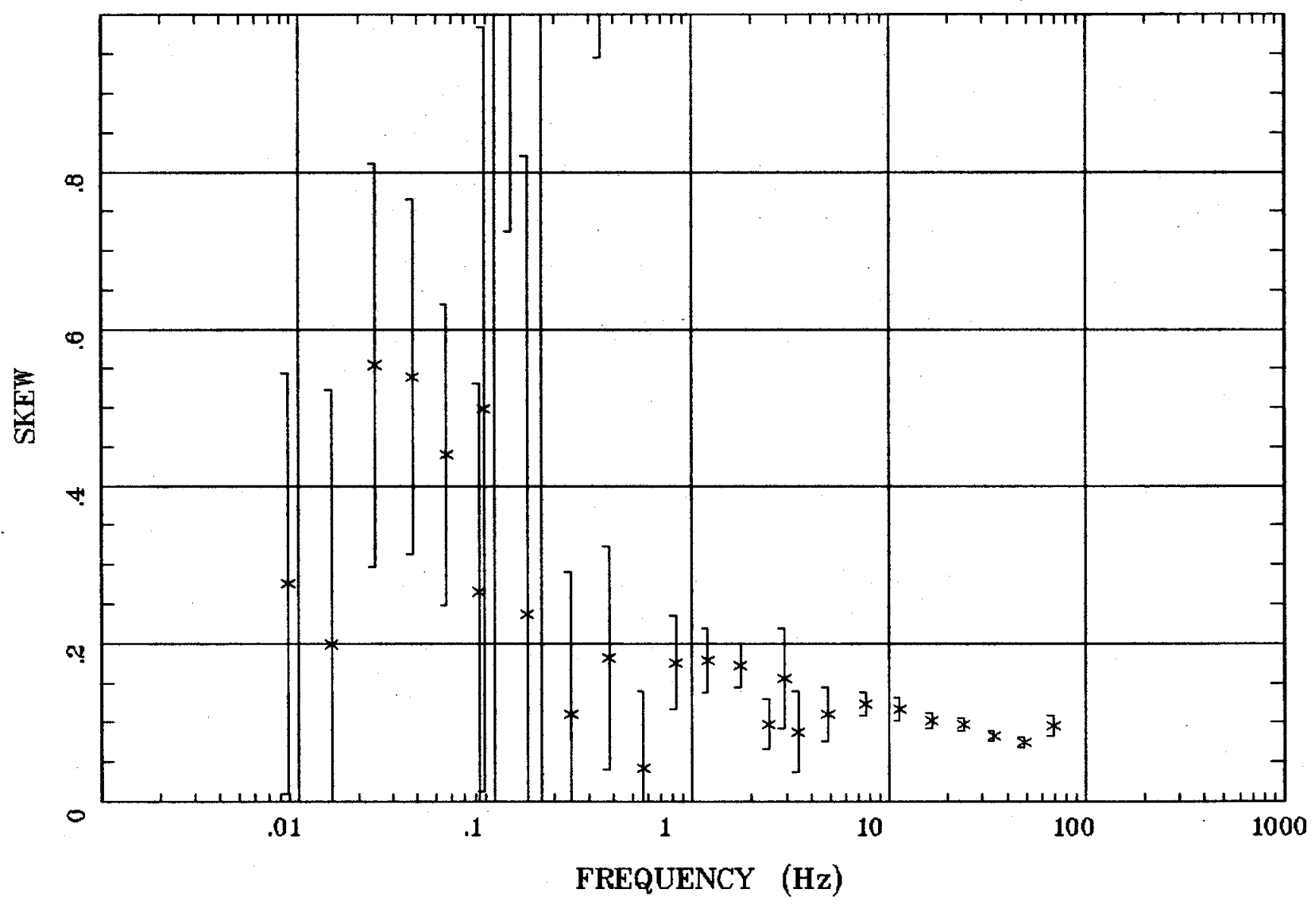

Client:

Remote: none

Acquired: 09:4 Aug 07, 1999

Survey Co:USGS

Rotation:

Filen ame: ar83b.avg

Channels: Ch1 Ch2 Ch3 Ch4 Ch5 Ch3 Ch4

Plotted: 11:01 Dec 08, 2000

< EMI - ElectroMagnetic Instruments 


\section{Station 83}

E MULT Coh.

Diamond Mtns., NV

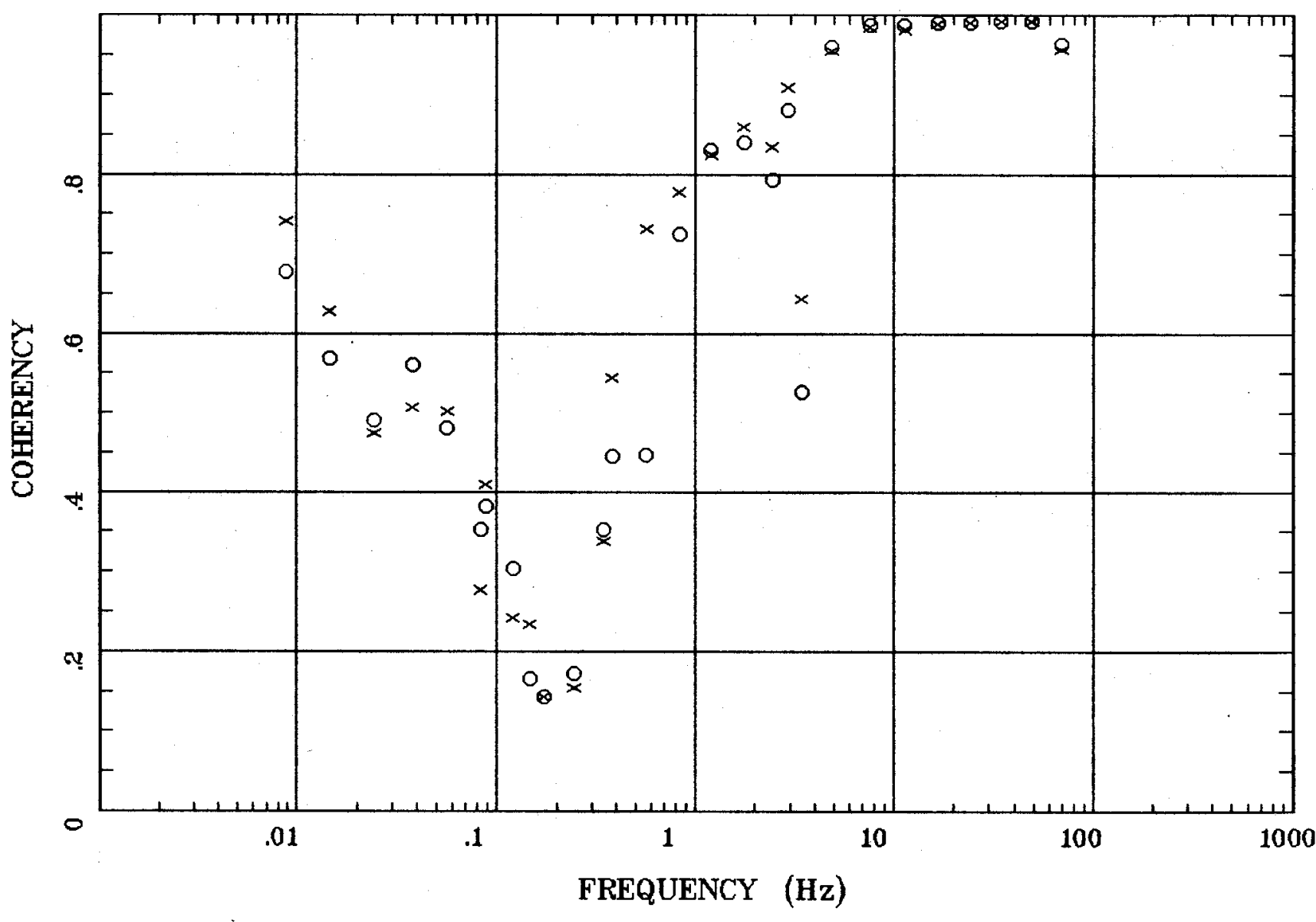

Client:

Remote: none

Acquired: 09:4 Aug 07, 1999

Survey Co:USGS
Rotation:

Filename: ar63b.avg

Channels: Ch1 Ch2 Ch3 Ch4 Ch5 Ch3 Ch4

Plotted: 11:01 Dec 08, 2000

< EMI - ElectroMagnetic Instruments 


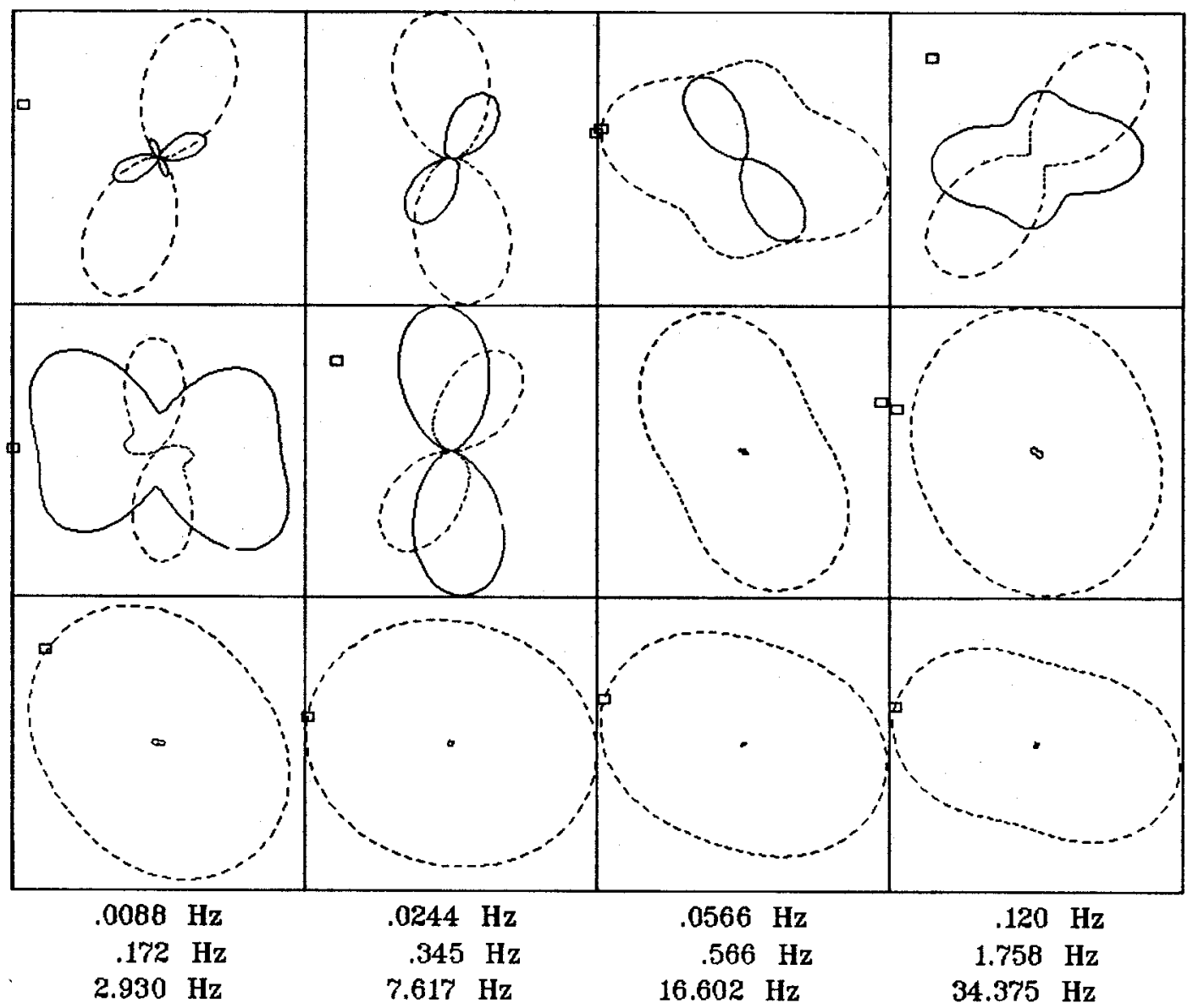

Client:

Remote: none

Acquired: 09:4 Aug 07, 1999 Survey Co:USGS
Rotation:

Filename: ar83b.avg

Channels: Ch1 Ch2 Ch3 Ch4 Ch5 Ch3 Ch4 Plotted: 11:01 Dec 08, 2000

< EMI - ElectroMagnetic Instruments > 


\section{Station 83}

TIPPER MAGNITUDE

Diamond Mtns., NV

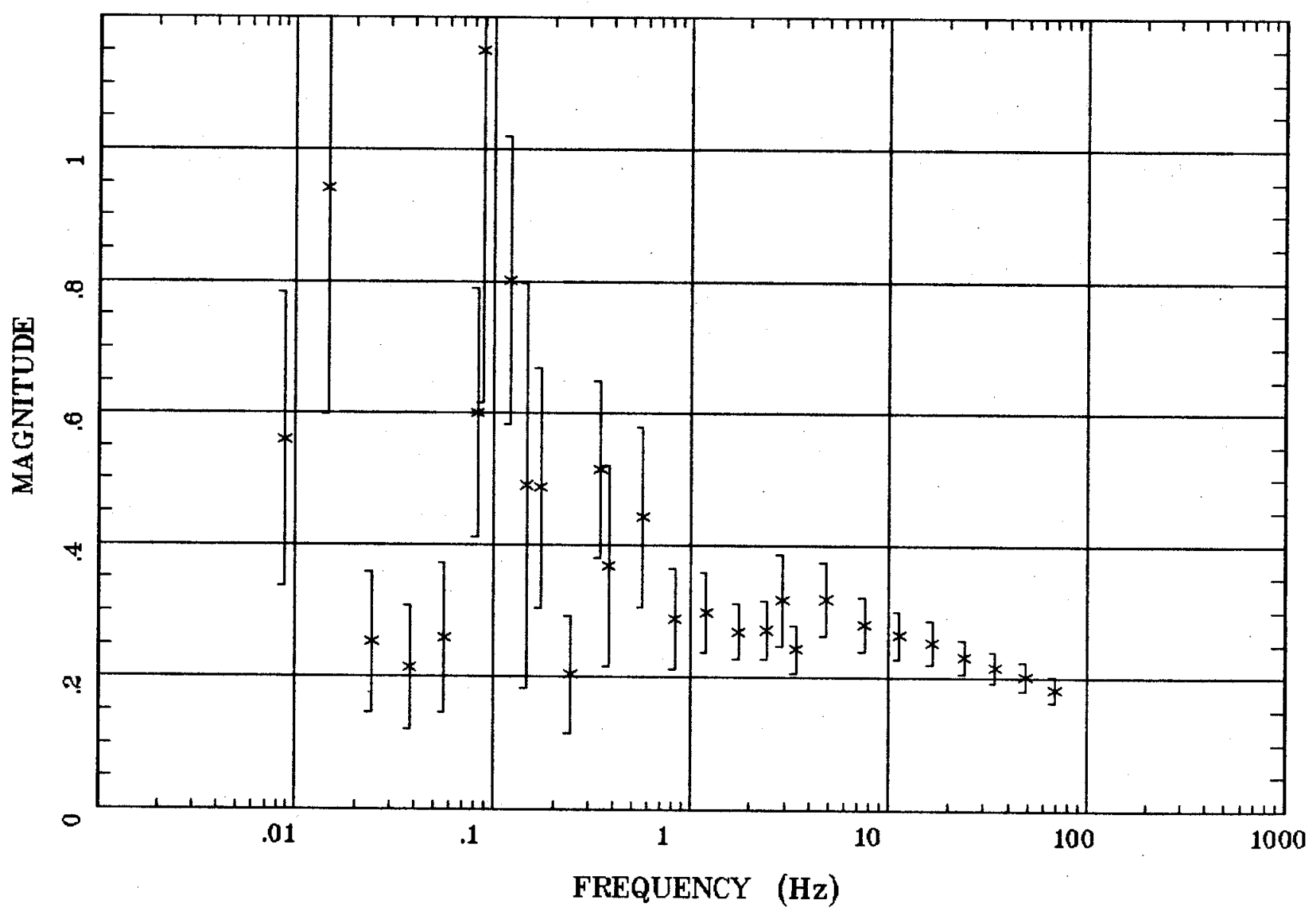

Client:

Remote: none

Acquired: 09:4 Aug 07, 1999

Survey Co:USGS
Rotation:

Filename: ar83b.avg

Channels: Ch1 Ch2 Ch3 Ch4 Ch5 Ch3 Ch4

Plotted: 11:01 Dec 08, 2000

< EMI - ElectroMagnetic Instruments > 
Diamond Mtns., NV

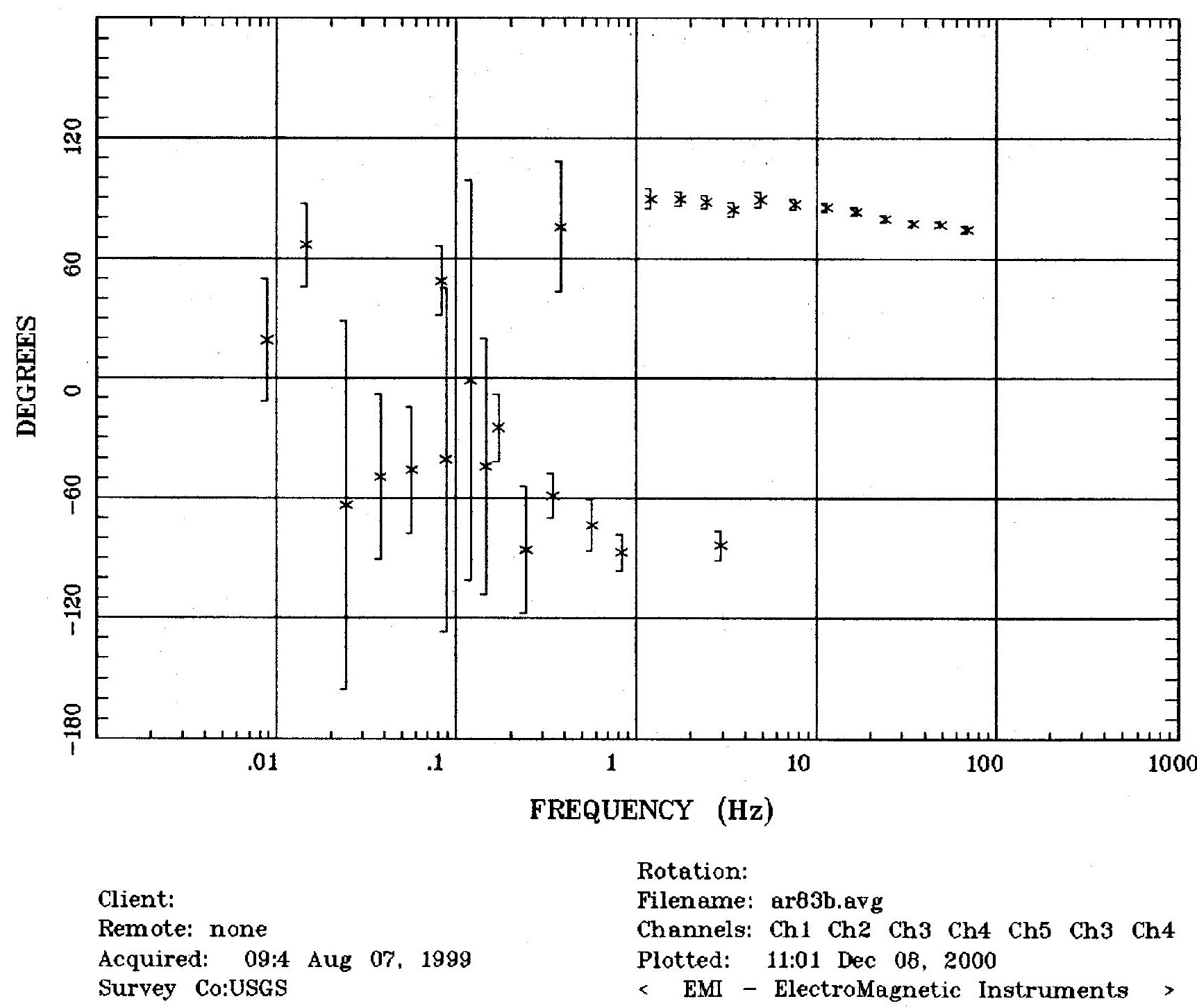


HzHx.x Coh HzHy.o

Diamond Mtns., NV

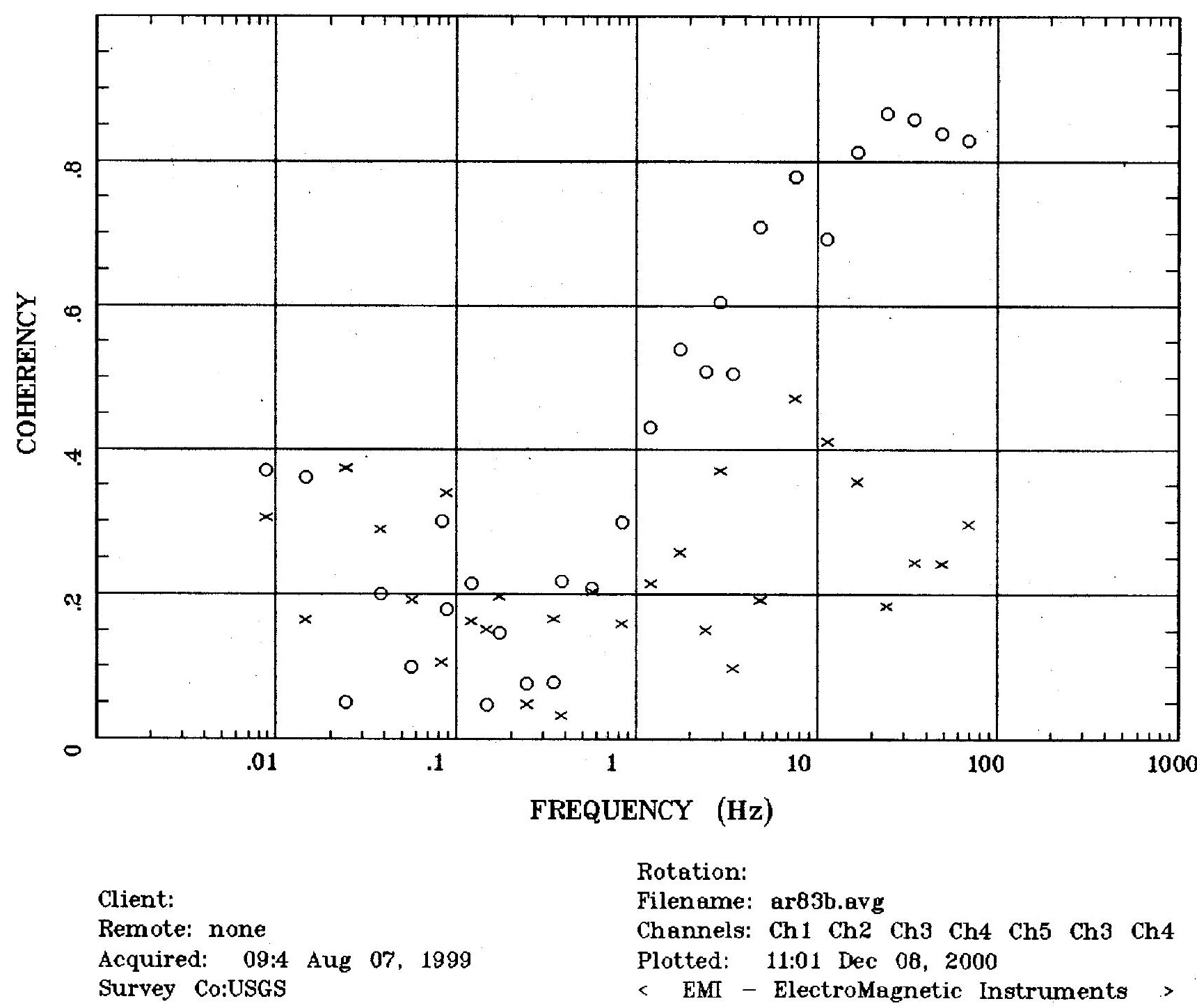




\section{Station $\mathbf{8 2}$}

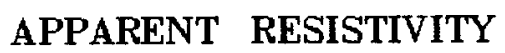

New ark Valley, NV

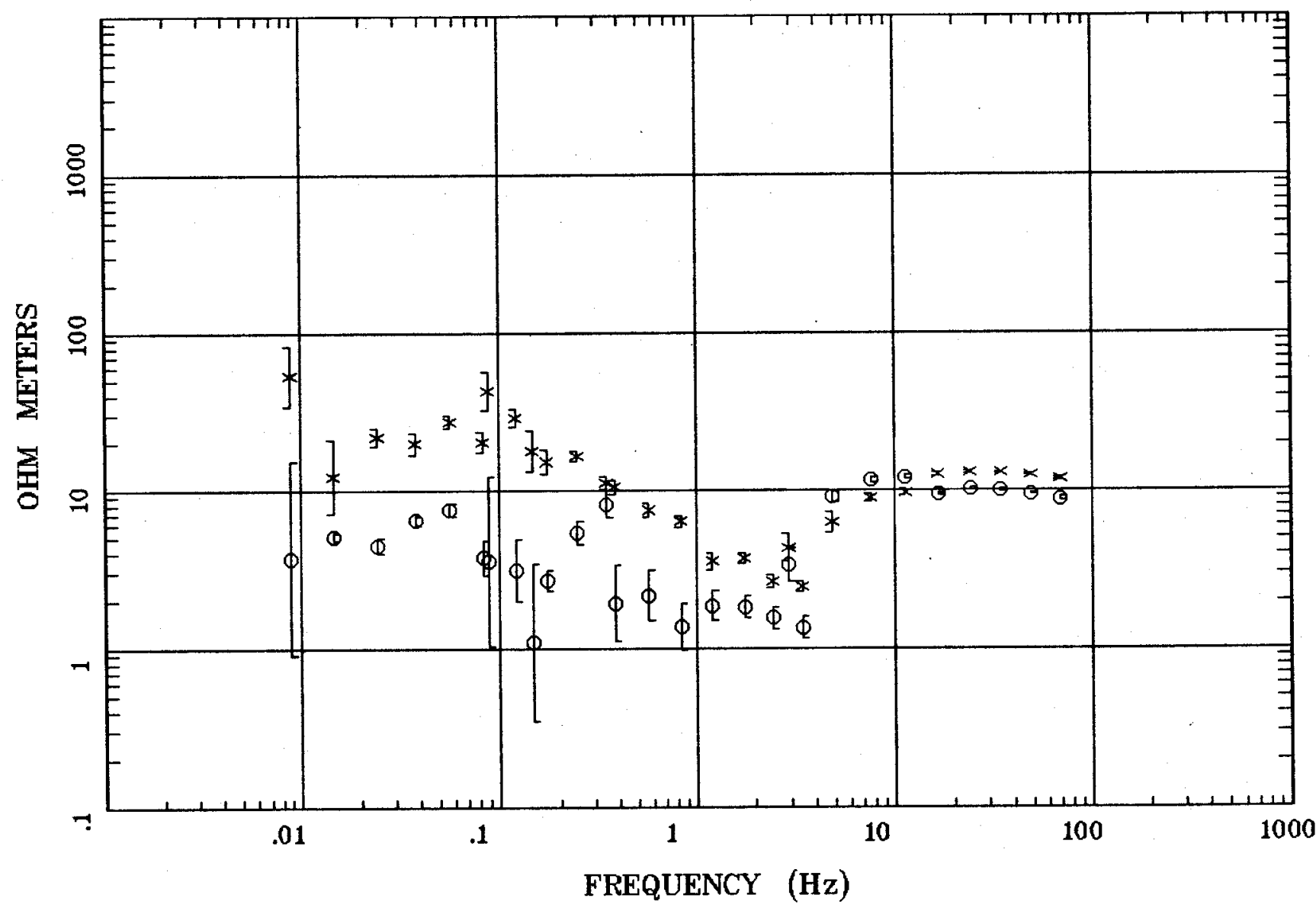

Client:

Remote: none

Acquired: 14:1 Aug 06, 1999 Survey Co:USGS
Rotation:

Filename: ar82b.avg

Channels: Ch1 Ch2 Ch3 Ch4 Ch5 Ch3 Ch4 Plotted: 11:00 Dec 08, 2000

< EMI - ElectroMagnetic Instruments 
IMPEDANCE PHASE

Newark Valley, NV

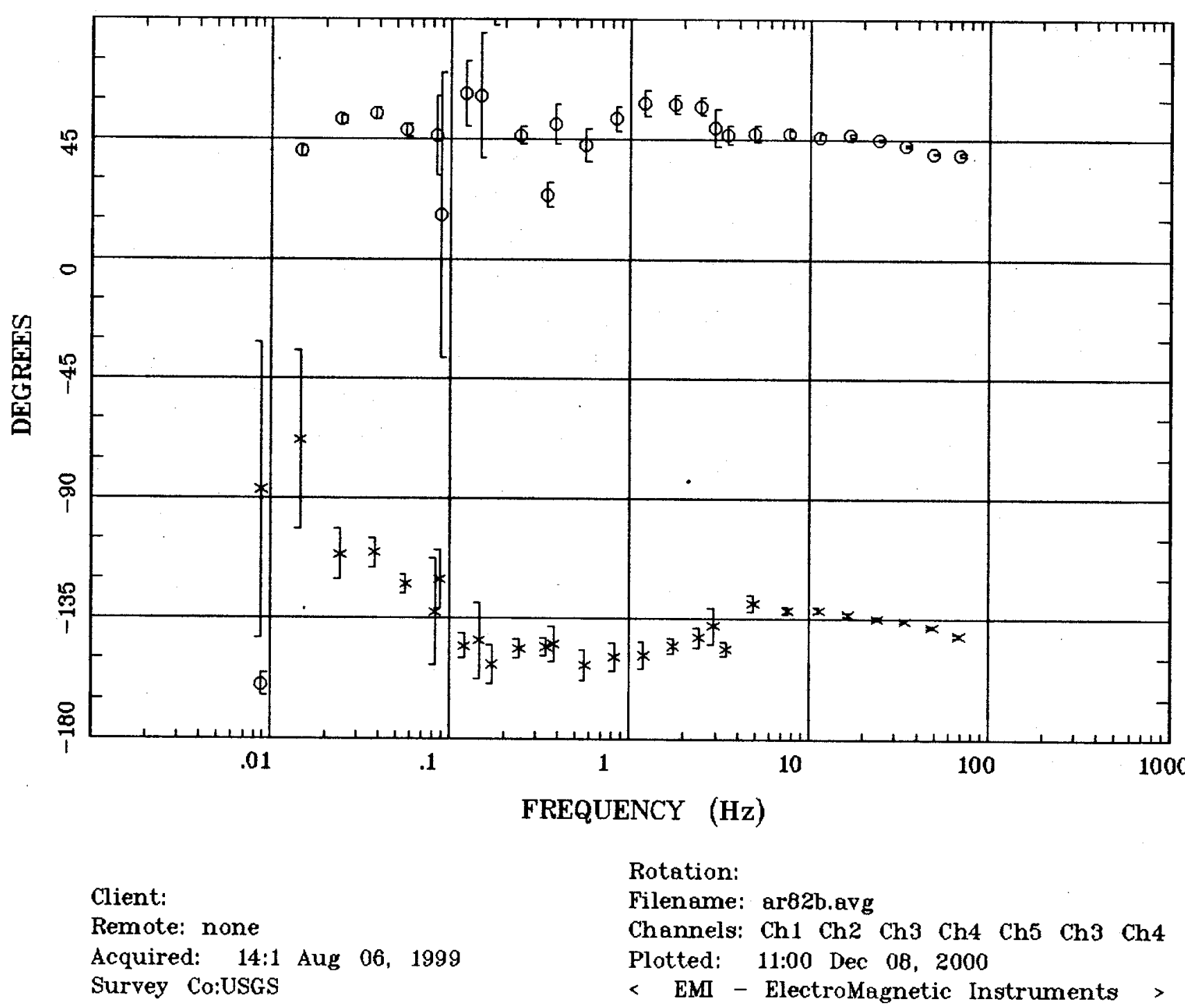


ROTATION ANGLE

Newark Valley, NV

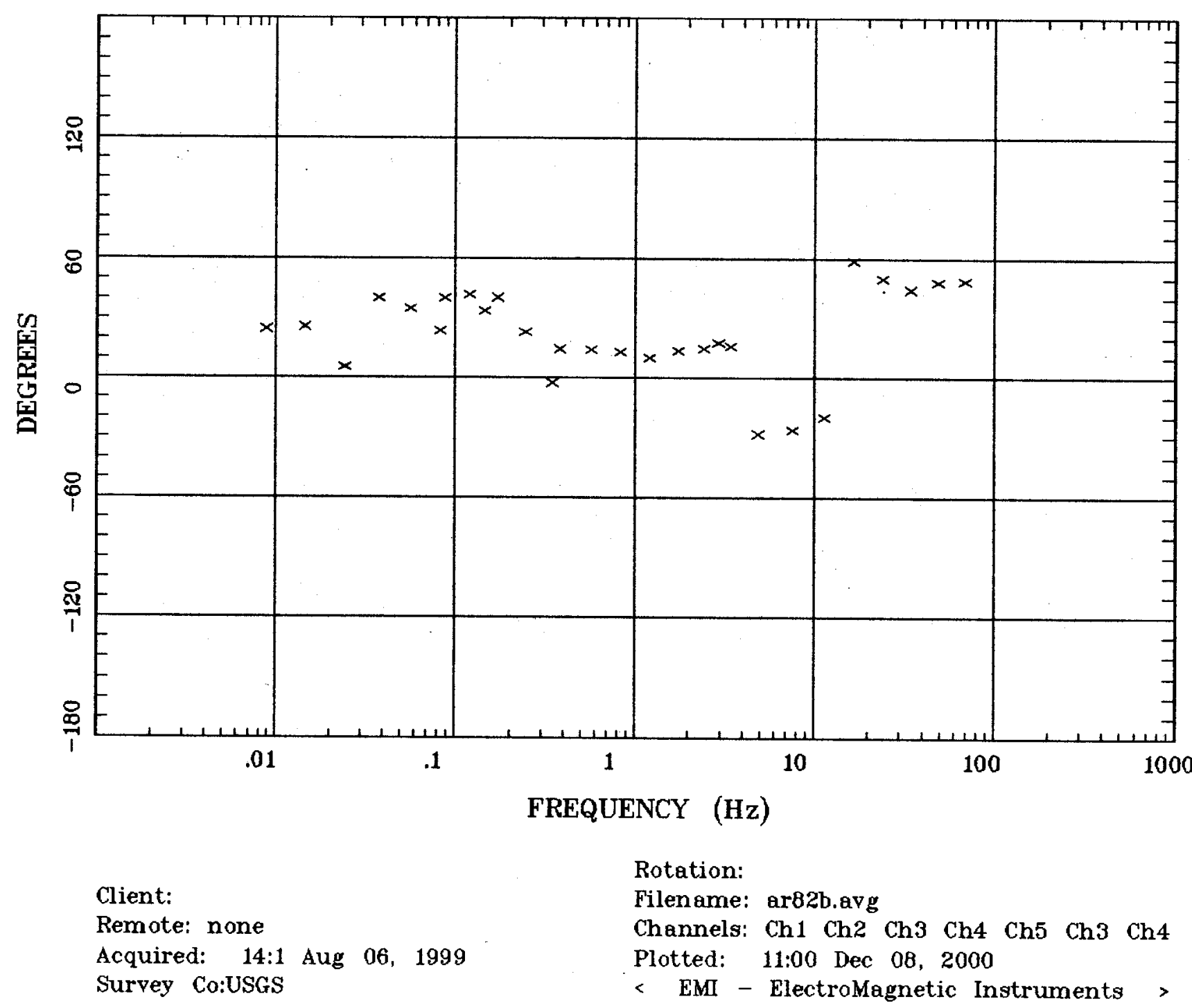




\section{Station 82}

IMPEDANCE SKEW

New ark Valley, NV

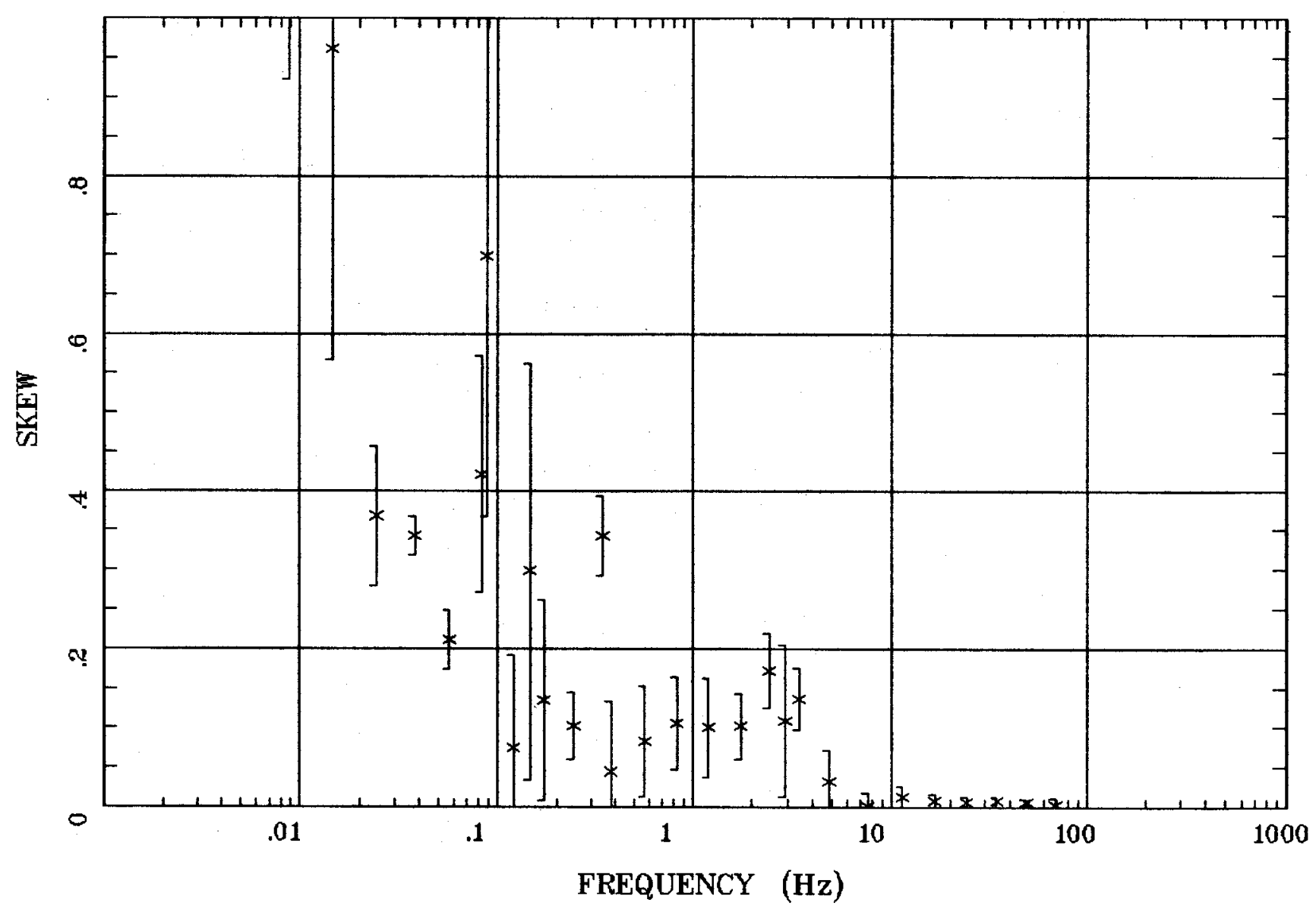

\section{Client:}

Remote: none

Acquired: 14:1 Aug 06, 1999

Rotation:

Filename: ar82b.avg

Channels: Ch1 Ch2 Ch3 Ch4 Ch5 Ch3 Ch4

Plotted: 11:00 Dec 08, 2000

Survey Co:USGS

< EMI - ElectroMagnetic Instruments 
E MULT Coh.

Newark Valley, NV

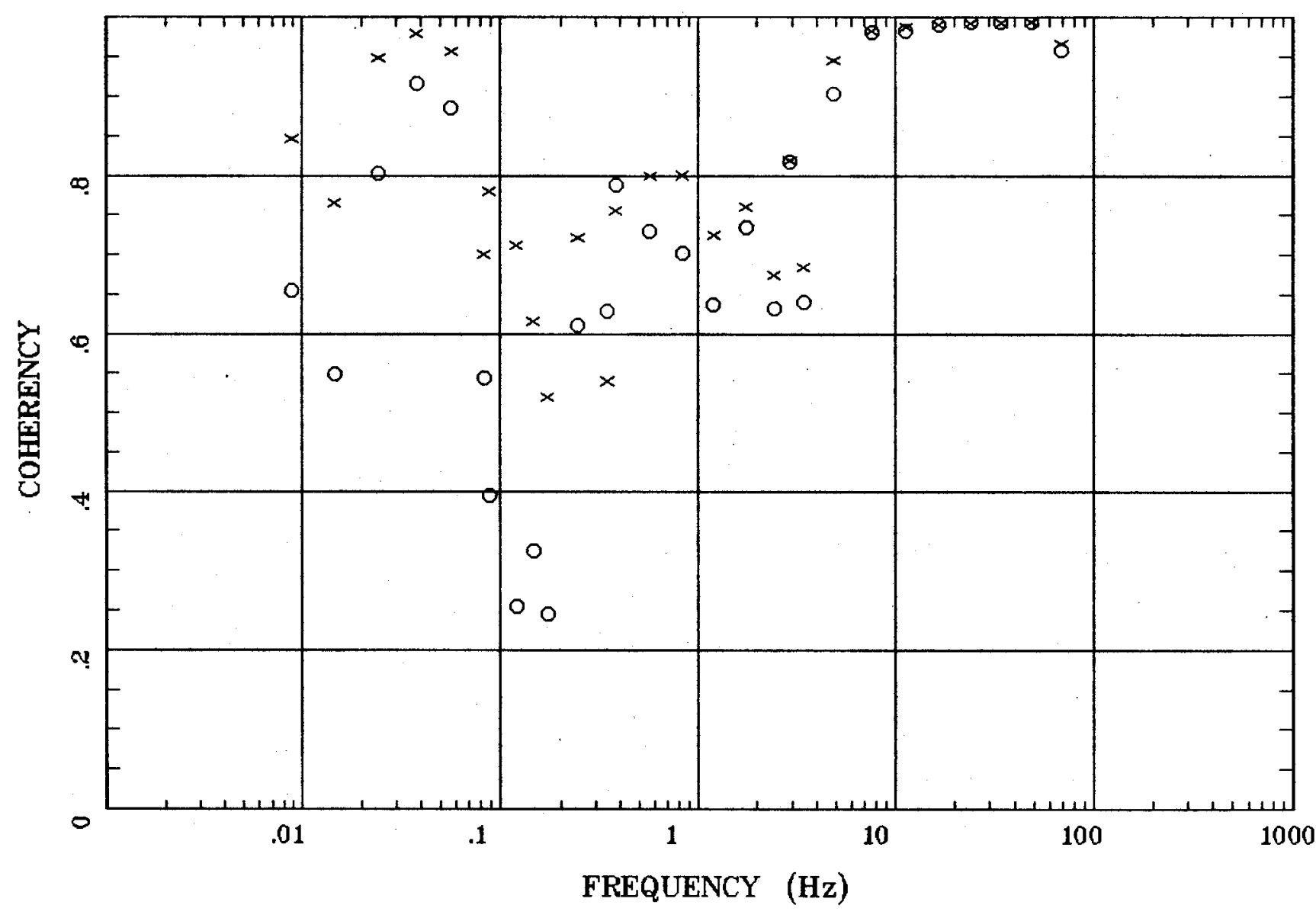

Client:

Remote: none

Acquired: 14:1 Aug 06, 1999 Survey Co:USGS
Rotation:

Filename: ar82b.avg

Channels: Ch1 Ch2 Ch3 Ch4 Ch5 Ch3 Ch4 Plotted: 11:00 Dec 08, 2000

< EMI - ElectroMagnetic Instruments 


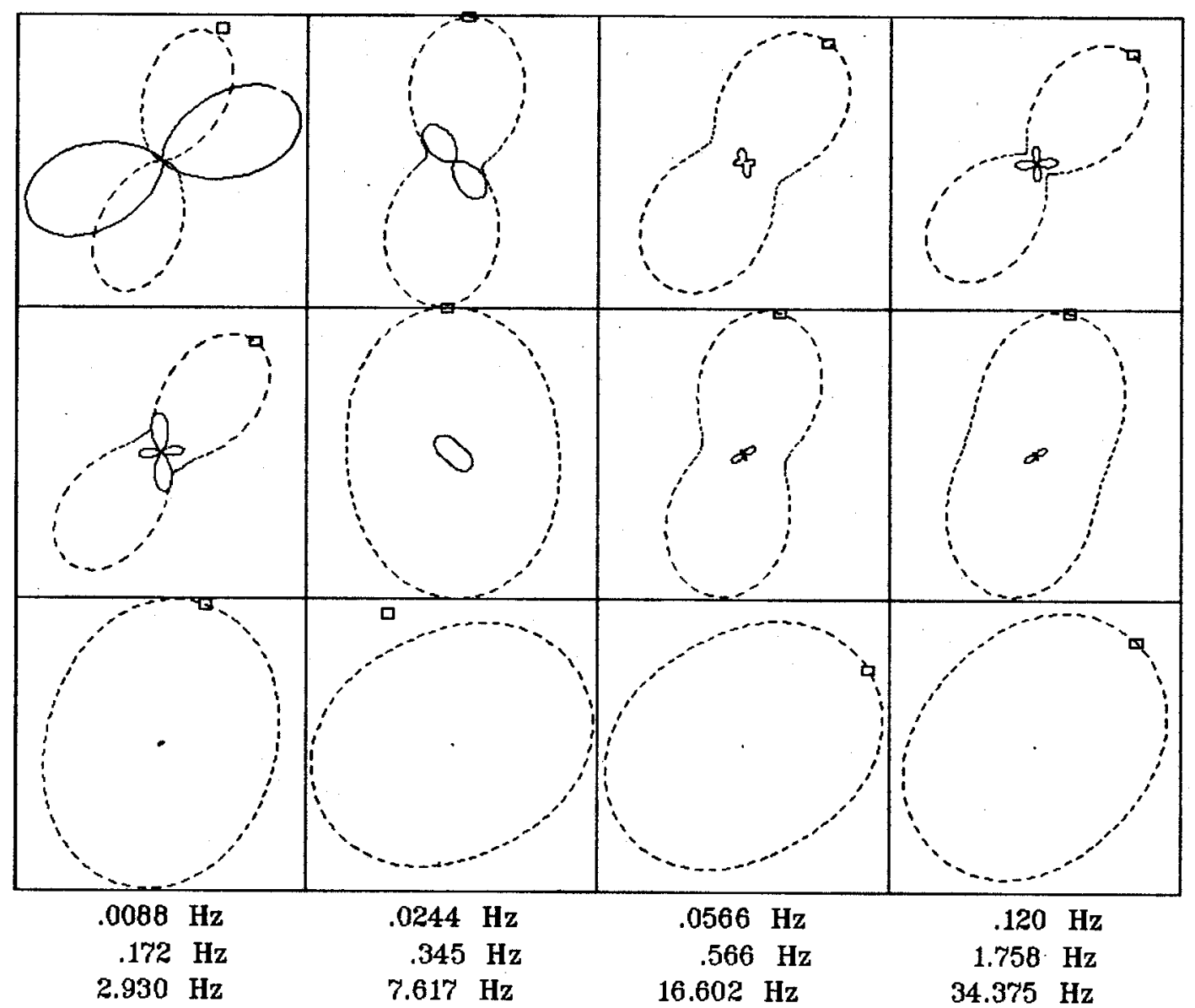

Client:

Remote: none

Acquired: 14:1 Aug 06, 1999 Survey Co:USGS
Rotation:

Filename: ar82b.avg

Channels: Ch1 Ch2 Ch3 Ch4 Ch5 Ch3 Ch4 Plotted: 11:00 Dec 08, 2000

$<$ EMI - ElectroMagnetic Instruments > 


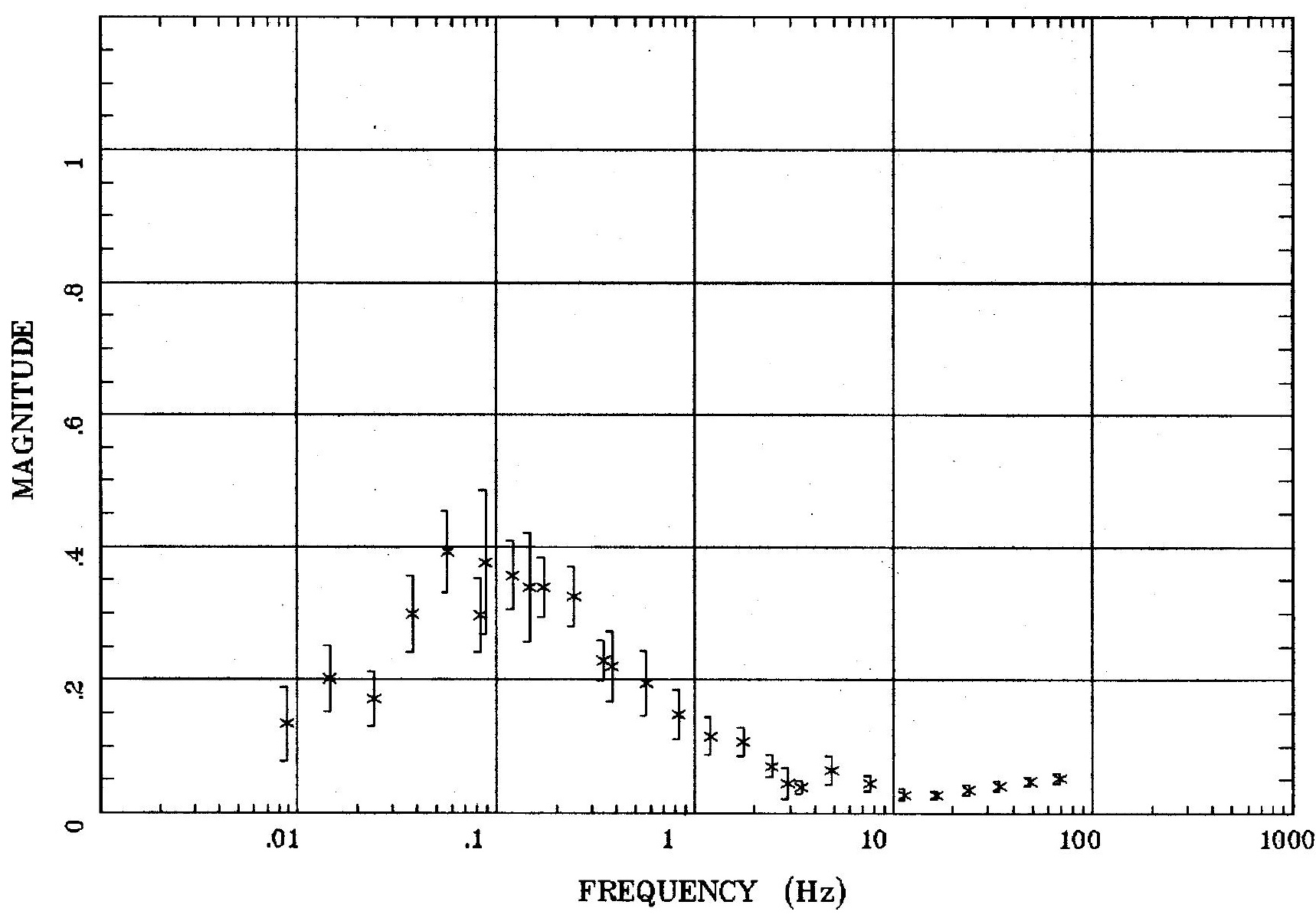

Client:

Remote: none

Acquired: 14:1 Aug 06, 1999 Survey Co:USGS
Rotation:

Filename: ar82b.avg

Channels: Ch1 Ch2 Ch3 Ch4 Ch5 Ch3 Ch4 Plotted: 11:00 Dec 08, 2000

$<$ EMI - ElectroMagnetic Instruments 


\section{Station 82}

TIPPER STRIKE

New ark Valley, NV

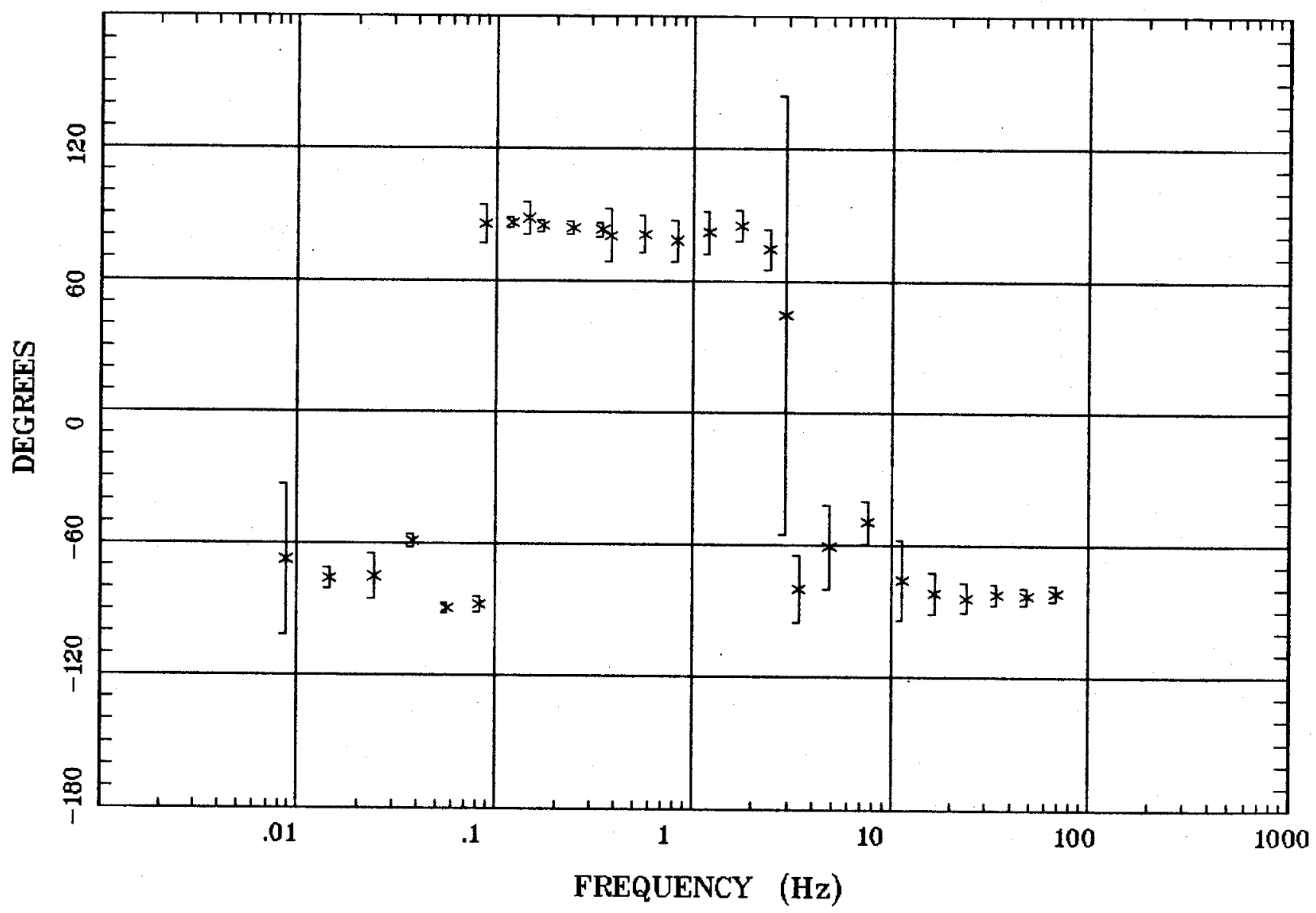

Client:

Remote: none

Acquired: 14:1 Aug 06, 1999

Survey Co:USGS
Rotation:

Filename: arB2b.avg

Channels: Ch1 Ch2 Ch3 Ch4 Ch5 Ch3 Ch4

Plotted: 11:00 Dec 08, 2000

< EMI - ElectroMagnetic Instruments 
HzHx.x Coh HzHy.o

New ark Valley, NV

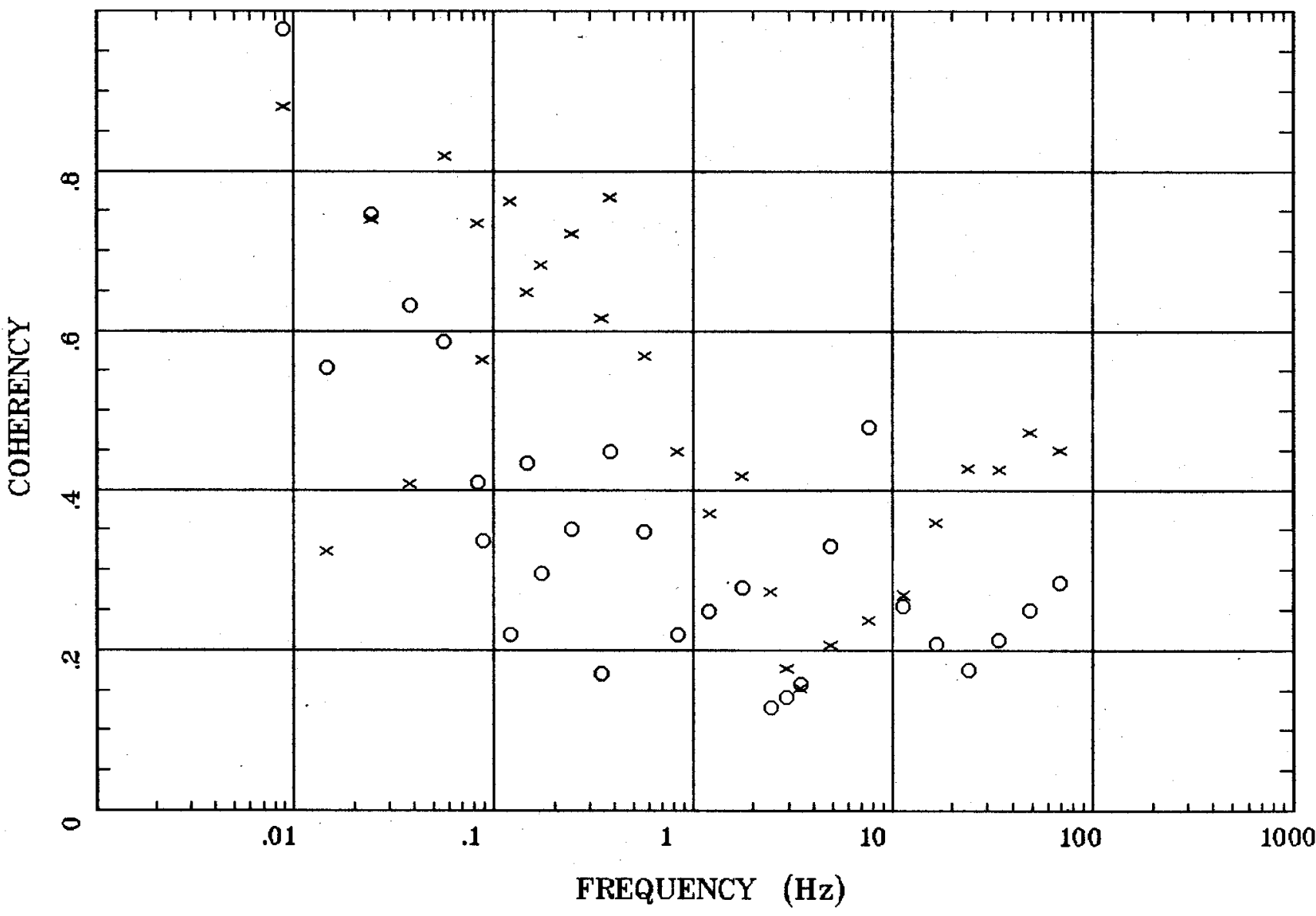

Client:

Remote: none

Acquired: 14:1 Aug 06, 1999

Survey Co:USGS
Rotation:

Filename: ar82b.evg

Channels: Ch1 Ch2 Ch3 Ch4 Ch5 Ch3 Ch4 Plotted: 11:00 Dec 08, 2000

\& EMI - ElectroMagnetic Instruments > 
Station 81

APPARENT RESISTIVITY

newark valley,nv

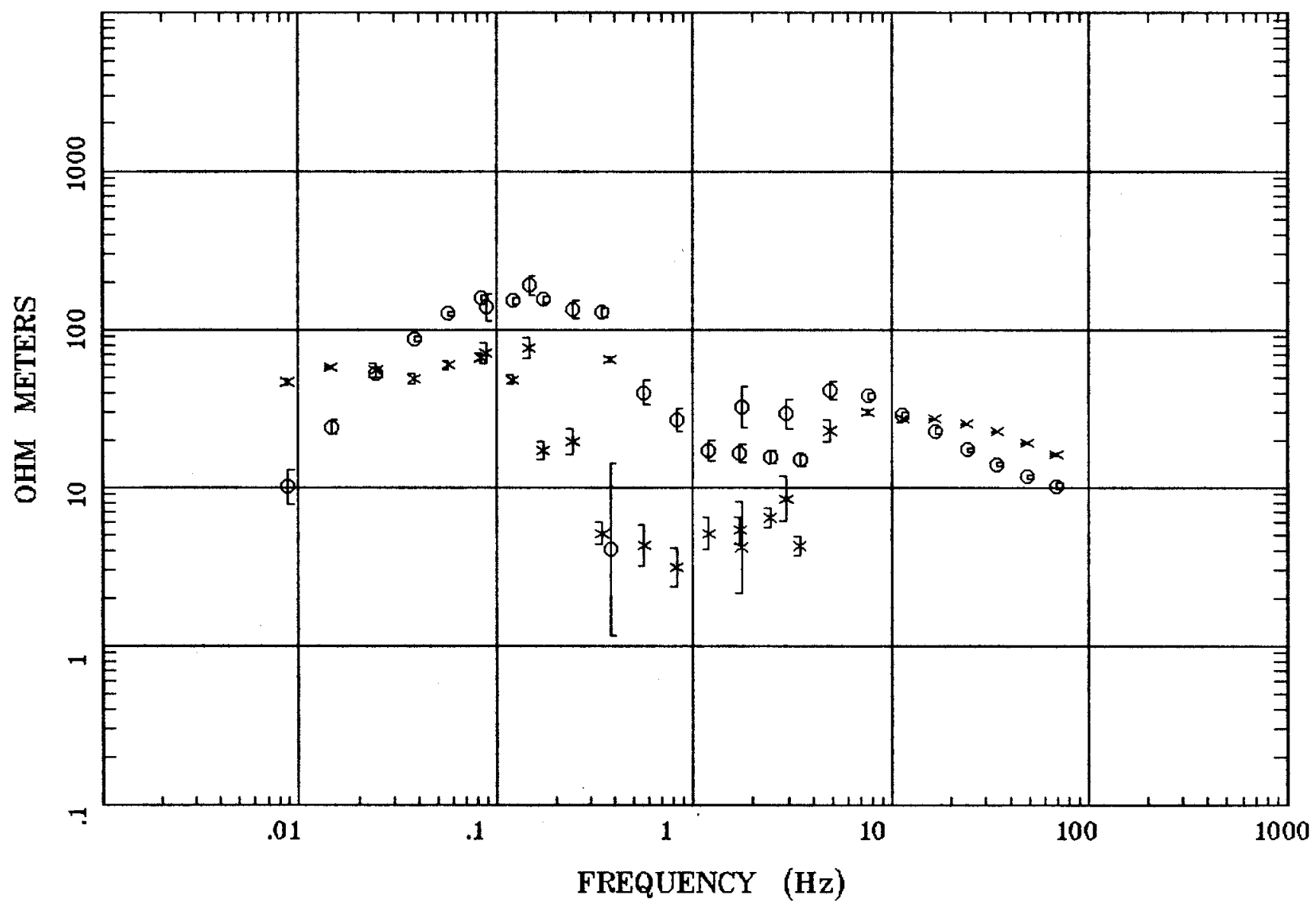

Client:

Remote: none

Acquired: 11:2 Aug 06, 1999 Survey Ca:USGS
Rotation:

Filename: ar81.avg

Channels: Ch1 Ch2 Ch3 Ch4 Ch5 Ch3 Ch4

Platted: 10:58 Dec 08, 2000

< EMI - ElectroMagnetic Instruments > 


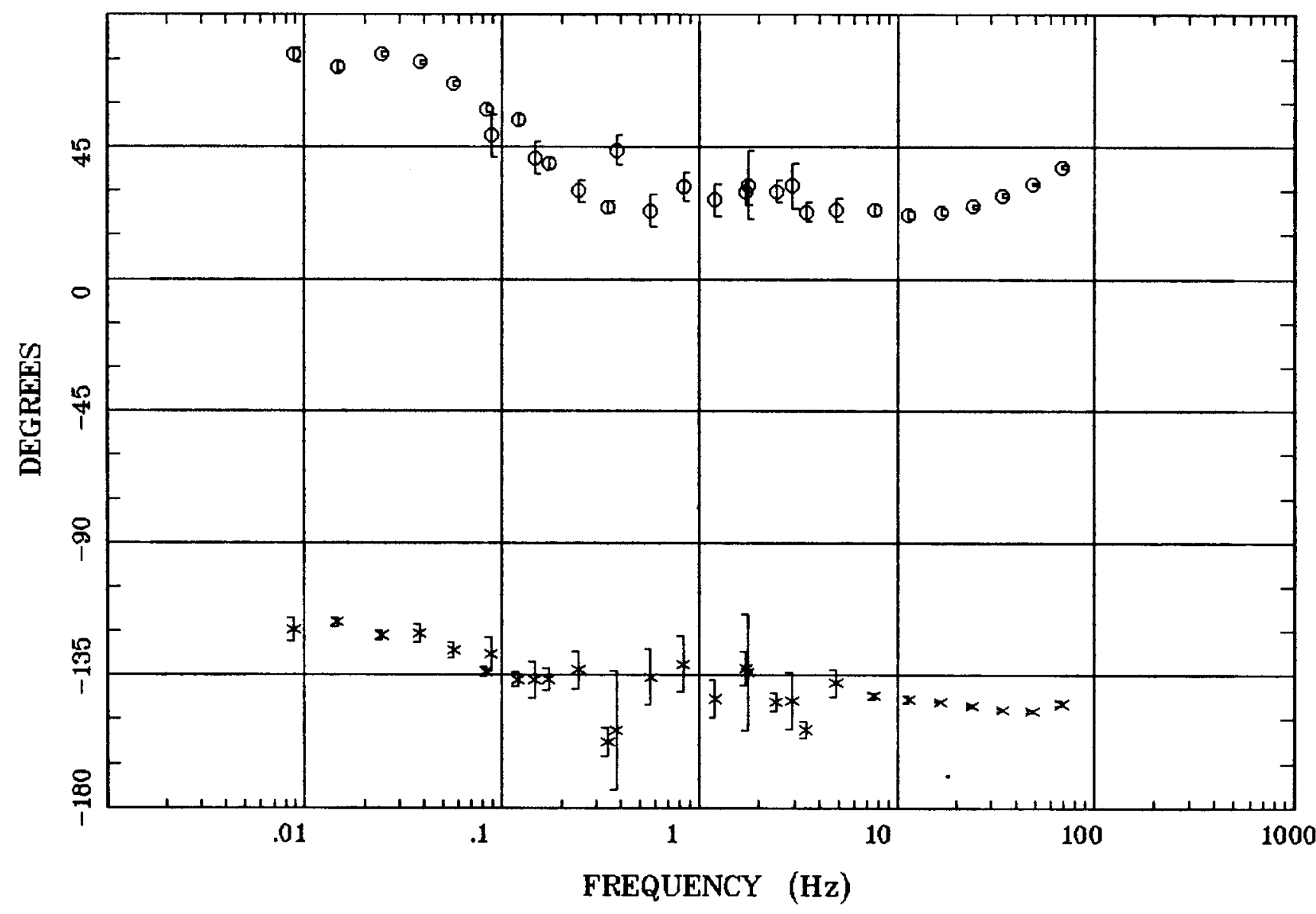

Client:

Remote: none

Acquired: 11:2 Aug 06, 1999

Survey Co:USGS
Rotation:

Filename: ar81.avg

Channels: Ch1 Ch2 Ch3 Ch4 Ch5 Ch3 Ch4

Plotted: 10:58 Dec 08, 2000

$<$ EMI - ElectroMagnetic Instruments 


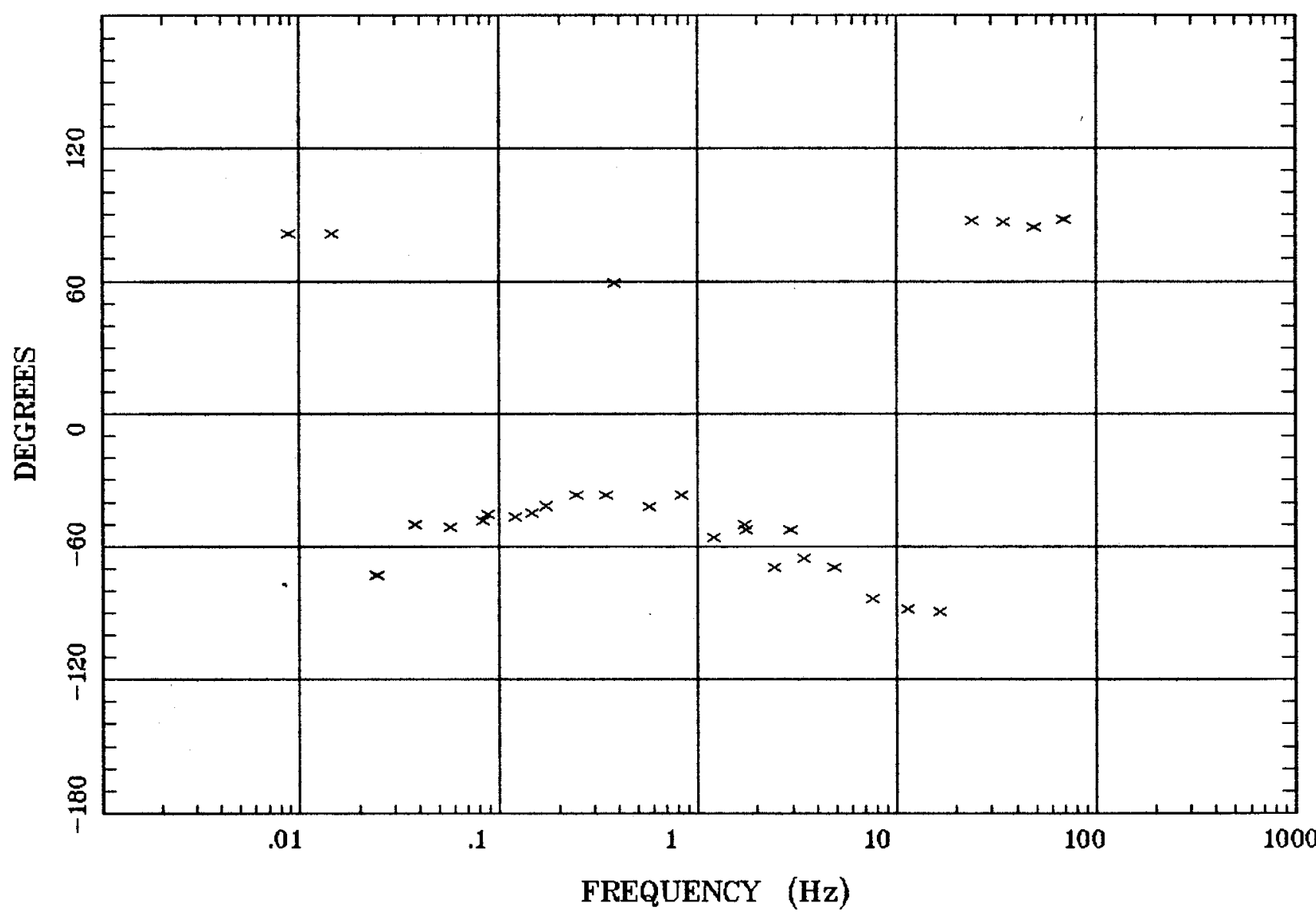

Client:

Remote: none

Acquired: 11:2 Aug 06, 1999

Survey Co:USGS
Rotation:

Filename: ar81.avg

Channels: Ch1 Ch2 Ch3 Ch4 Ch5 Ch3 Ch4

Plotted: 10:58 Dec 08, 2000

$<$ EMI - ElectroMagnetic Instruments 


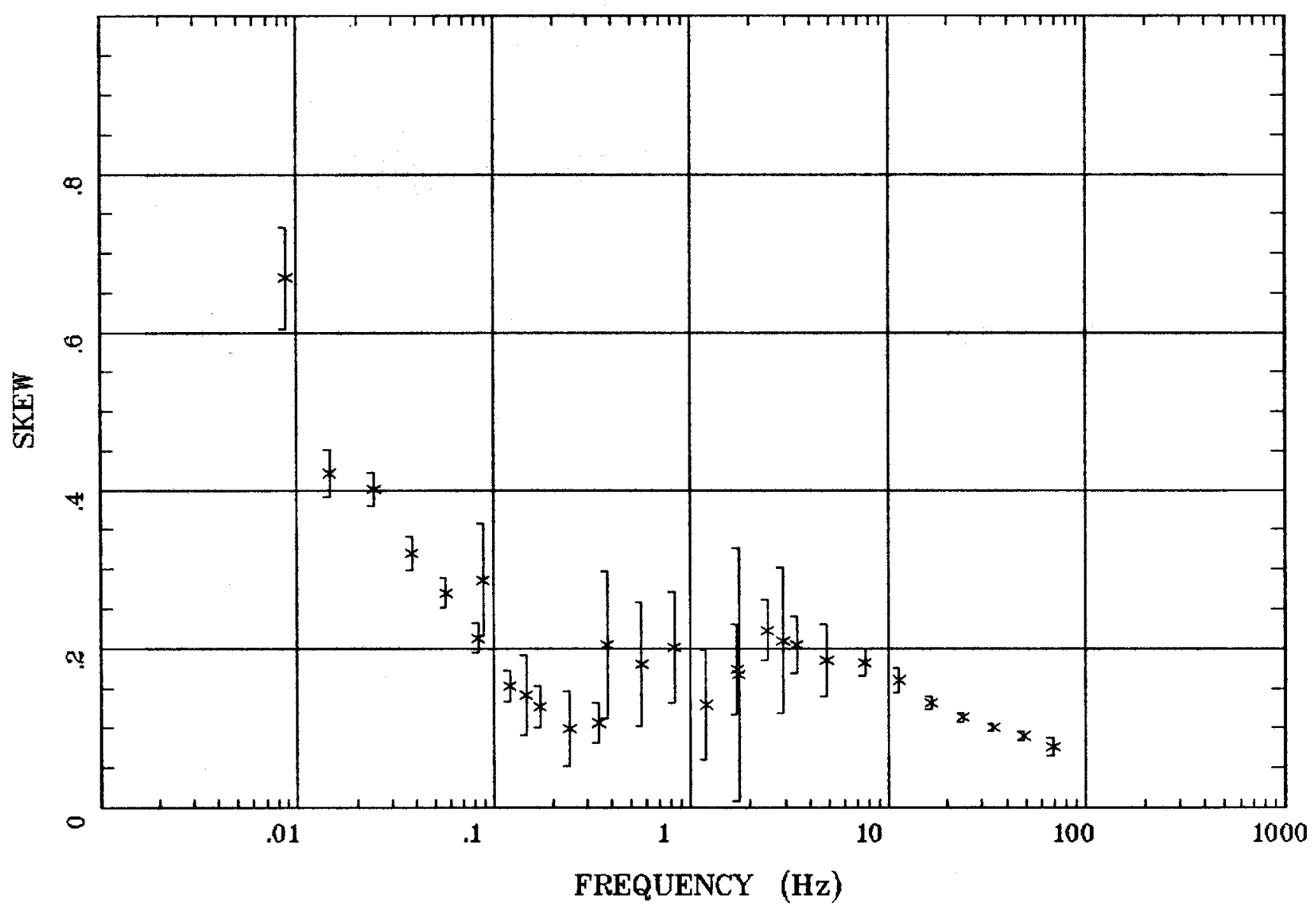

Client:

Remote: none

Acquired: 11:2 Aug 06, 1999

Survey Co:USGS

Rotation:

Filename: ar81.avg

Channels: Ch1 Ch2 Ch3 Ch4 Ch5 Ch3 Ch4

Plotted: 10:58 Dec 08, 2000

$<$ EMI - ElectroMagnetic Instruments > 
E MULT Coh.

newark valley,nv

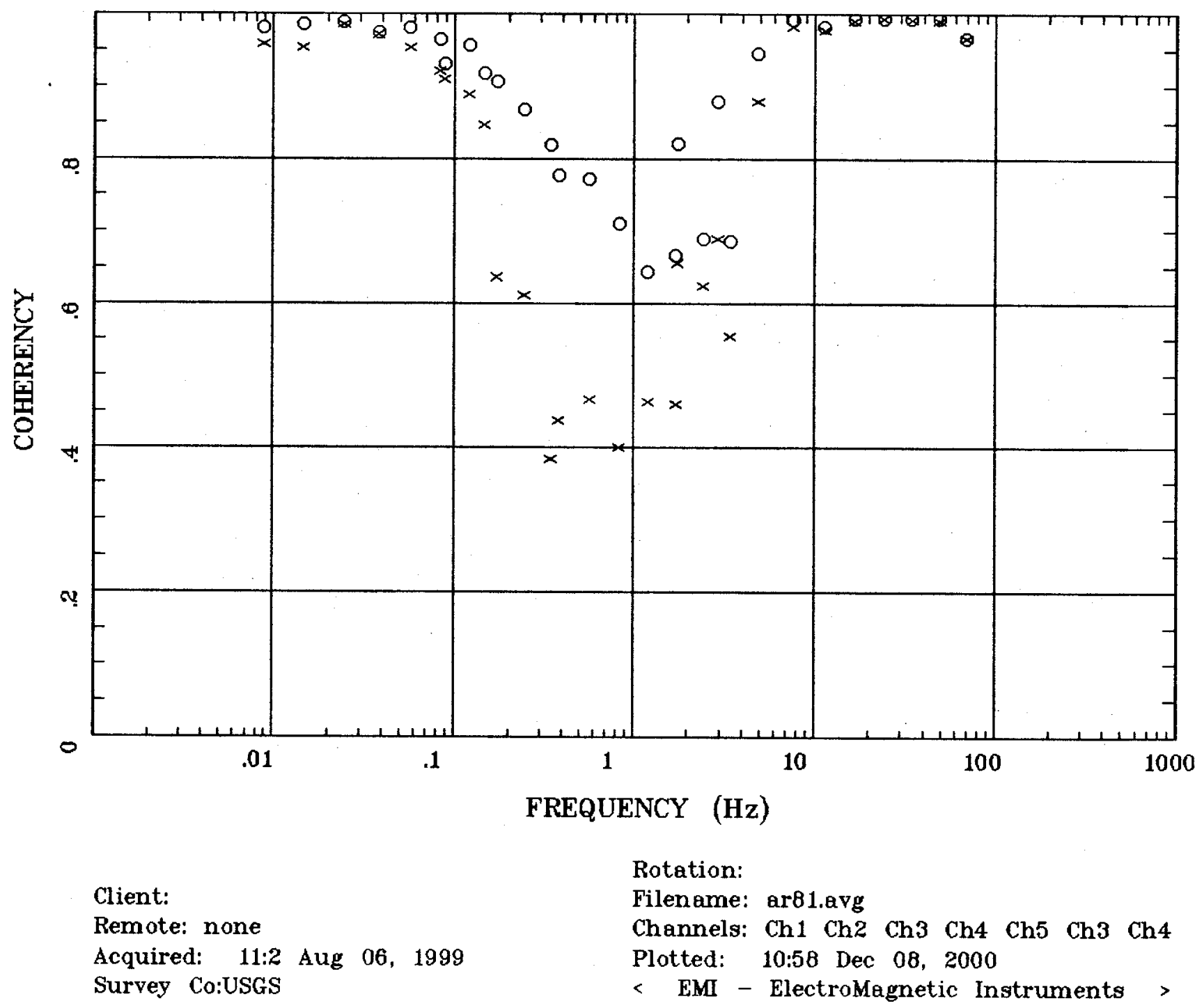




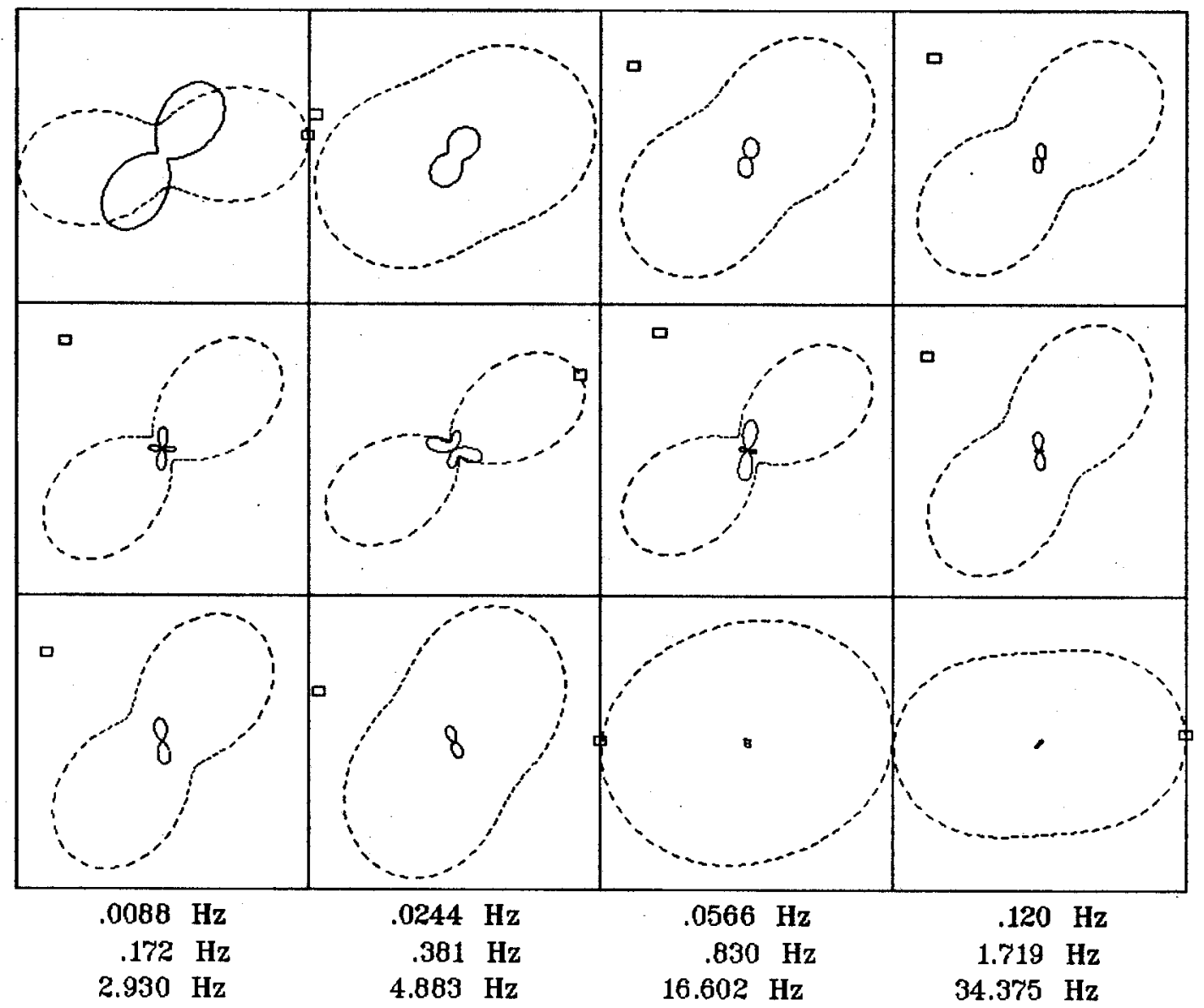

Client:

Remote: none

Acquired: 11:2 Aug 06, 1999

Survey Co:USGS
Rotation:

Filename: ar81.avg

Channels: Ch1 Ch2 Ch3 Ch4 Ch5 Ch3 Ch4 Plotted: 10:58 Dec 08, 2000

< EMI - ElectroMagnetic Instruments > 


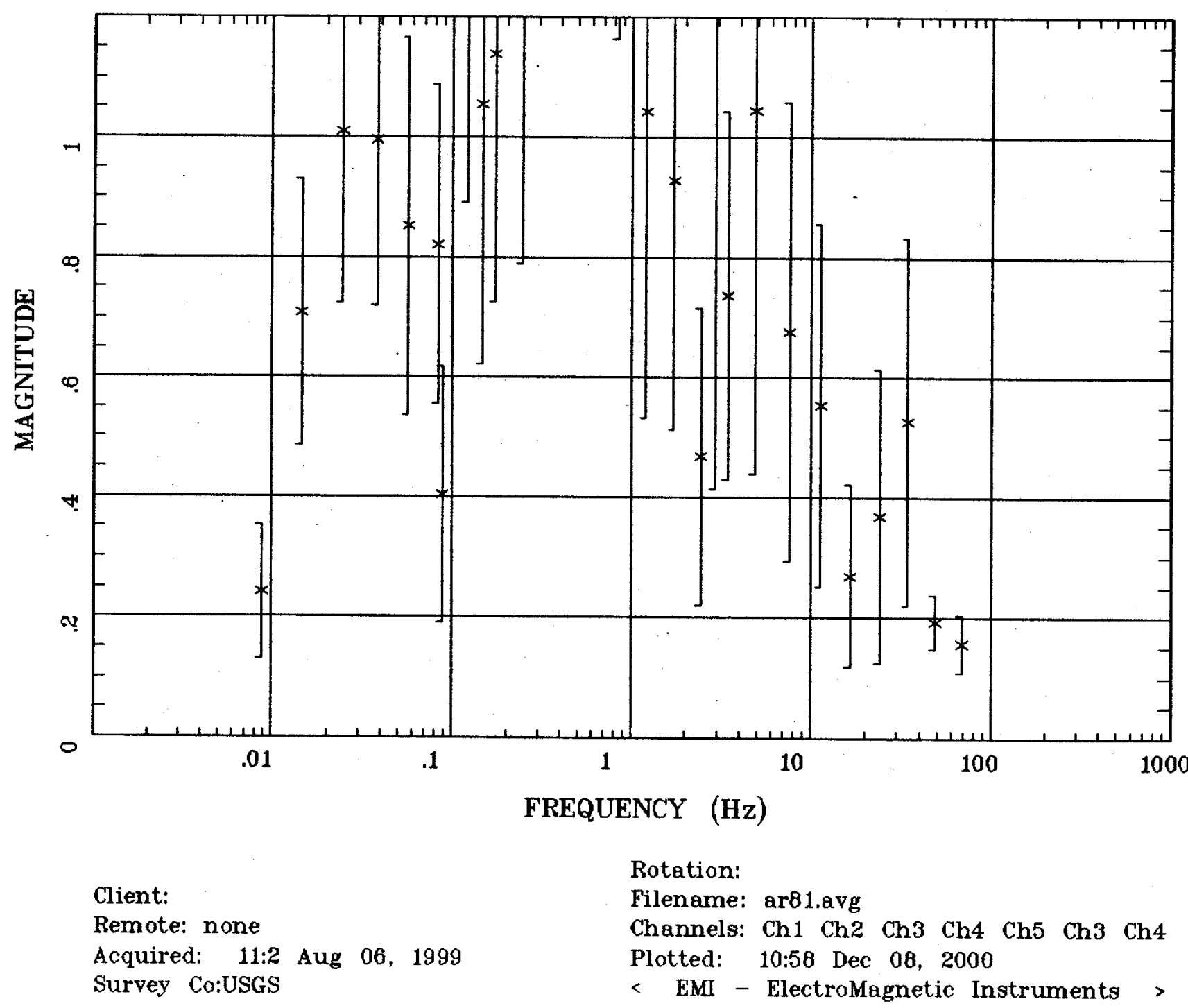




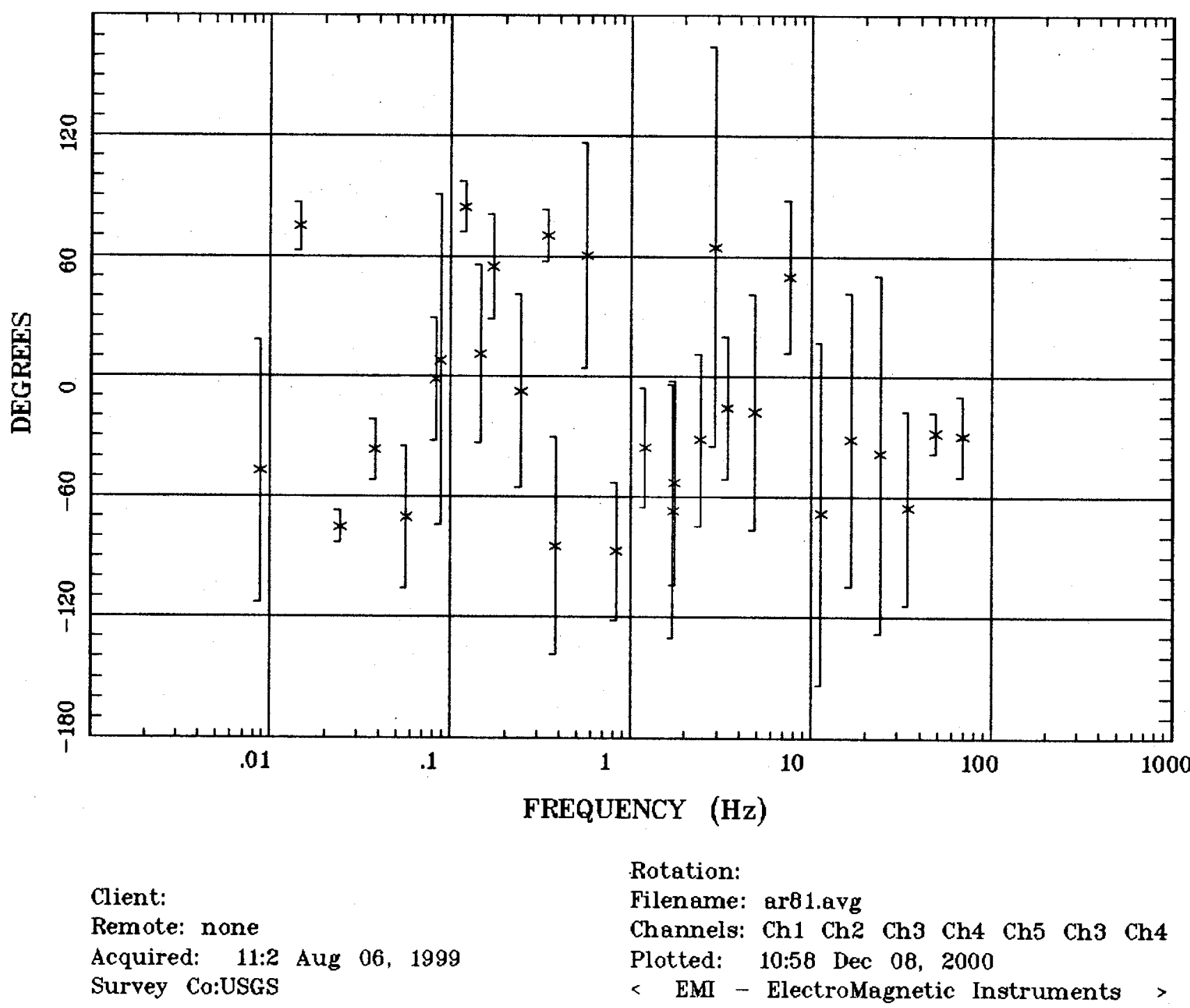


HzHx.x Coh HzHy.o newark valley,nv

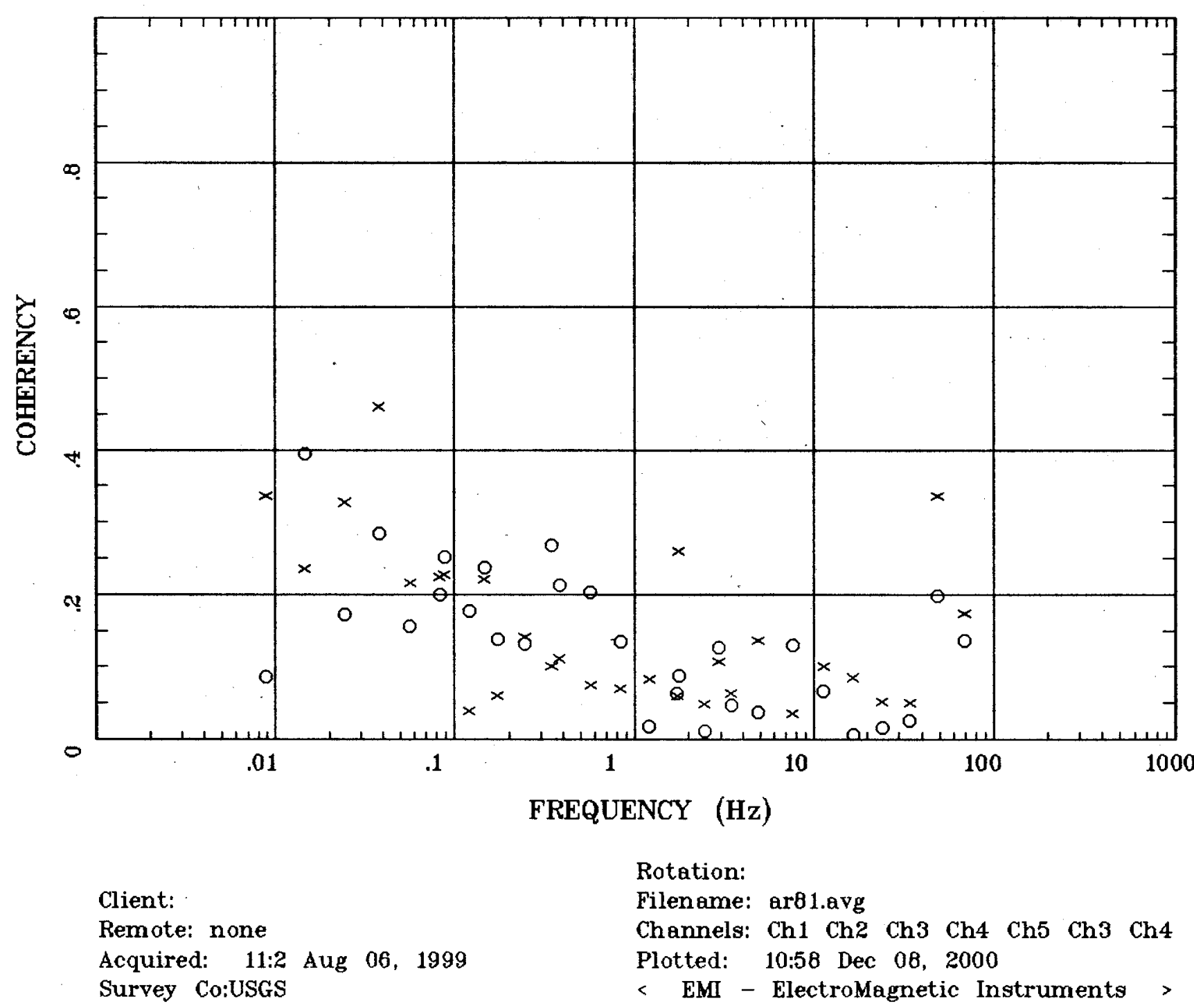




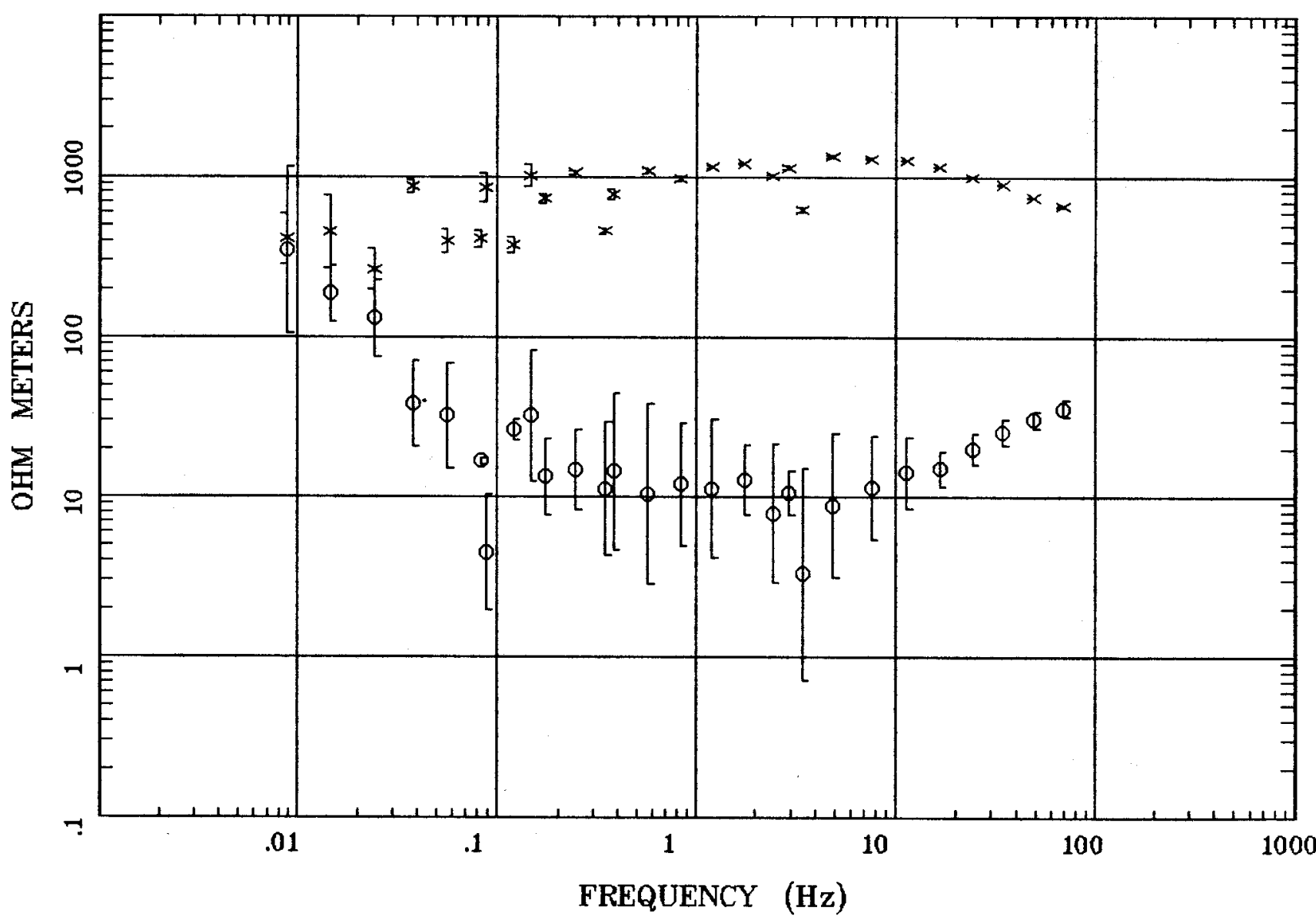

Client:

Remote: none

Acquired: 14:1 Aug 05, 1999 Survey Co:USGS
Rotation:

Filename: ar80a.avg

Channels: Ch1 Ch2 Ch3 Ch4 Ch5 Ch3 Ch4 Plotted: 10:57 Dec 08, 2000

< EMI - ElectroMagnetic Instruments > 


\section{IMPEDANCE PHASE}

Alligator Ridge

Station 80

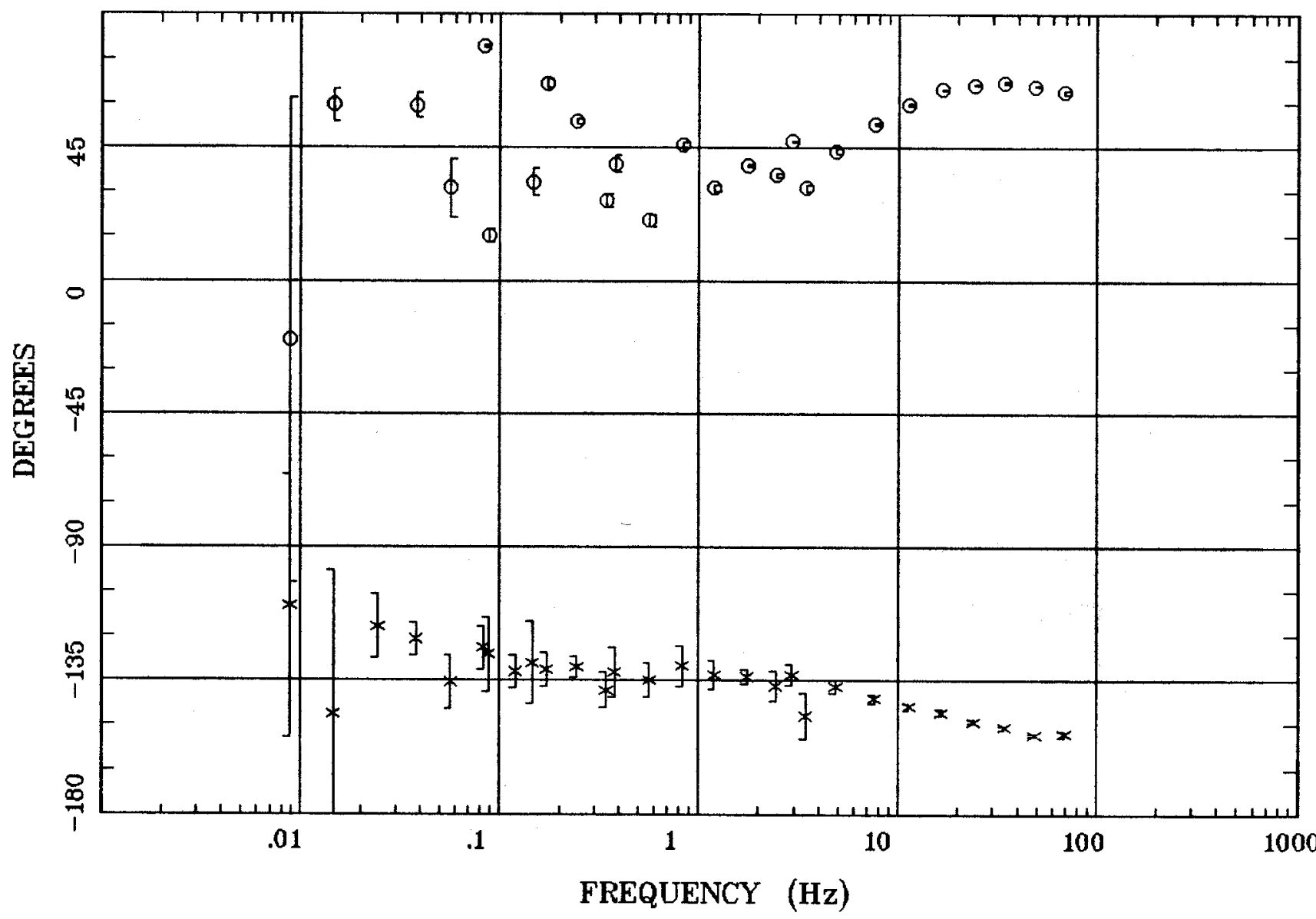

Client:

Remote: none

Acquired: 14:1 Aug 05, 1999 Survey Co:USGS
Rotation:

Filename: ar80a.avg

Channels: Ch1 Ch2 Ch3 Ch4 Ch5 Ch3 Ch4 Plotted: 10:57 Dec 08, 2000

< EMI - ElectroMagnetic Instruments 


\section{ROTATION ANGLE}

Alligator Ridge

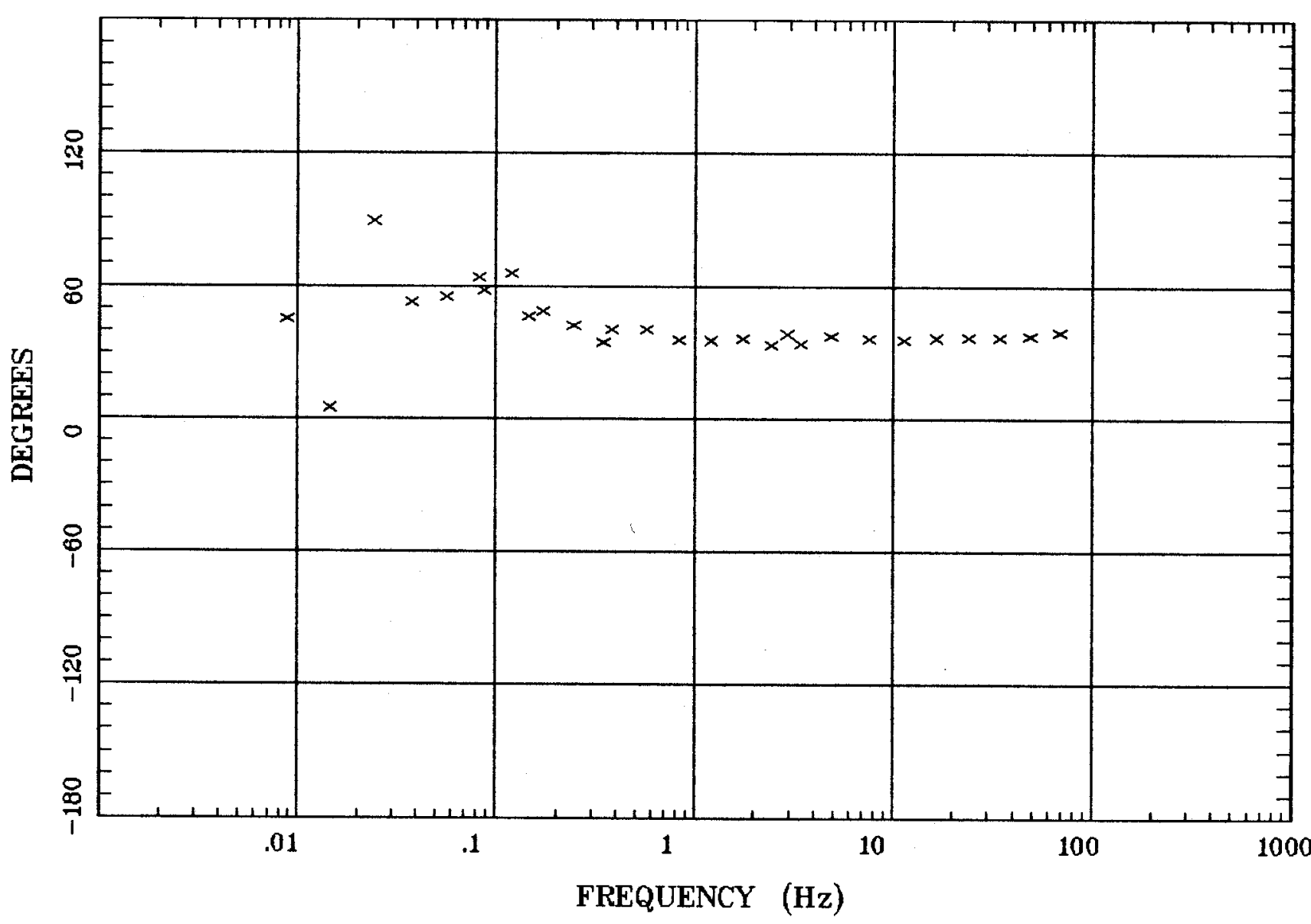

Client:

Remote: none

Acquired: 14:1 Aug 05, 1999

Survey Co:USGS
Rotation:

Filename: ar80a.avg

Channels: Ch1 Ch2 Ch3 Ch4 Ch5 Ch3 Ch4 Platted: 10:57 Dec 08, 2000

< EMI - ElectroMagnetic Instruments 


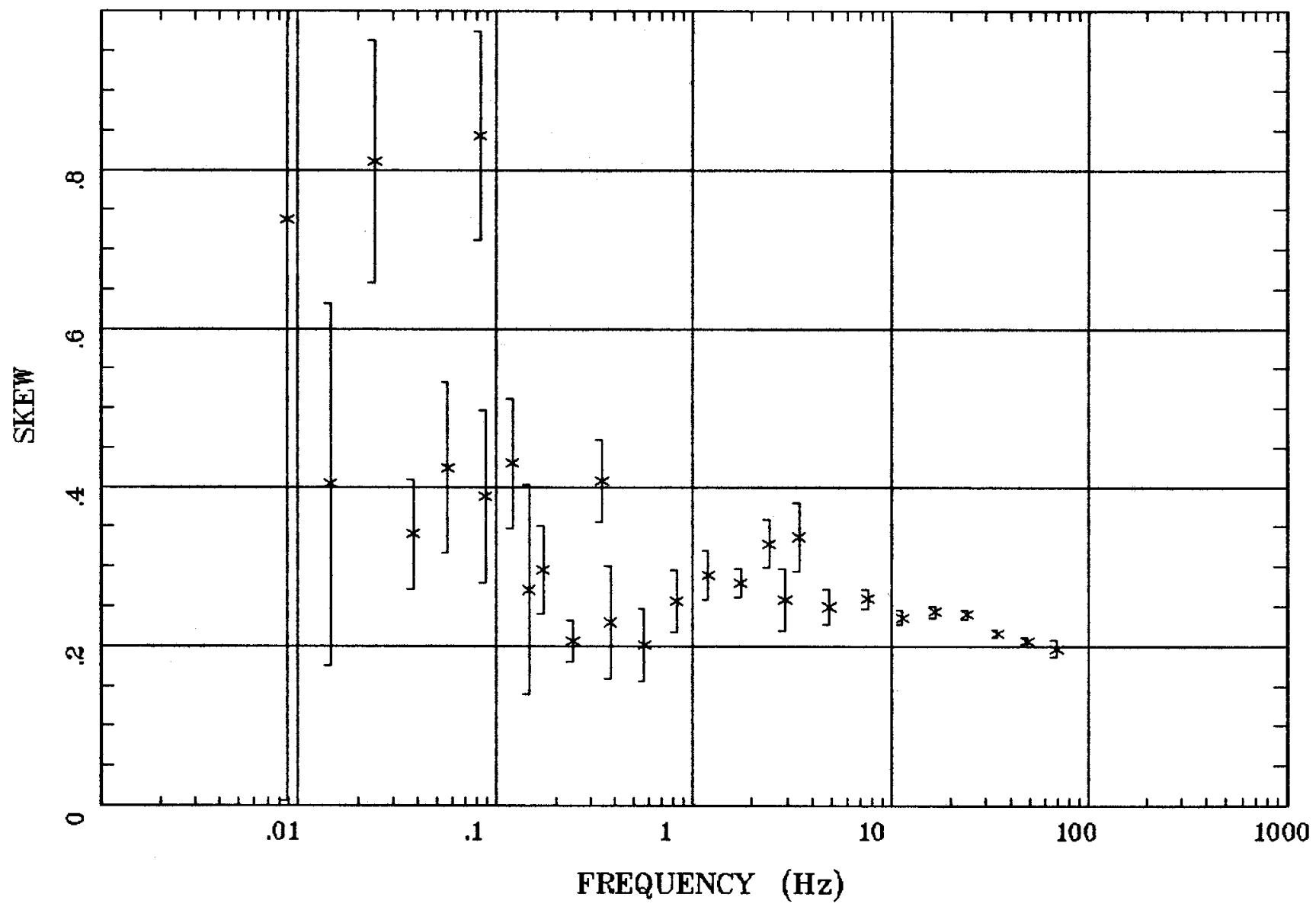

Client:

Remote: none

Acquired: 14:1 Aug 05, 1999 Survey Co:USGS
Rotation:

Filename: ar80a.avg

Channels: Ch1 Ch2 Ch3 Ch4 Ch5 Ch3 Ch4

Platted: 10:57 Dec 08, 2000

$<$ EMI - ElectroMagnetic Instruments 


\section{E MULT Coh.}

\section{Alligator Ridge}

Station 80

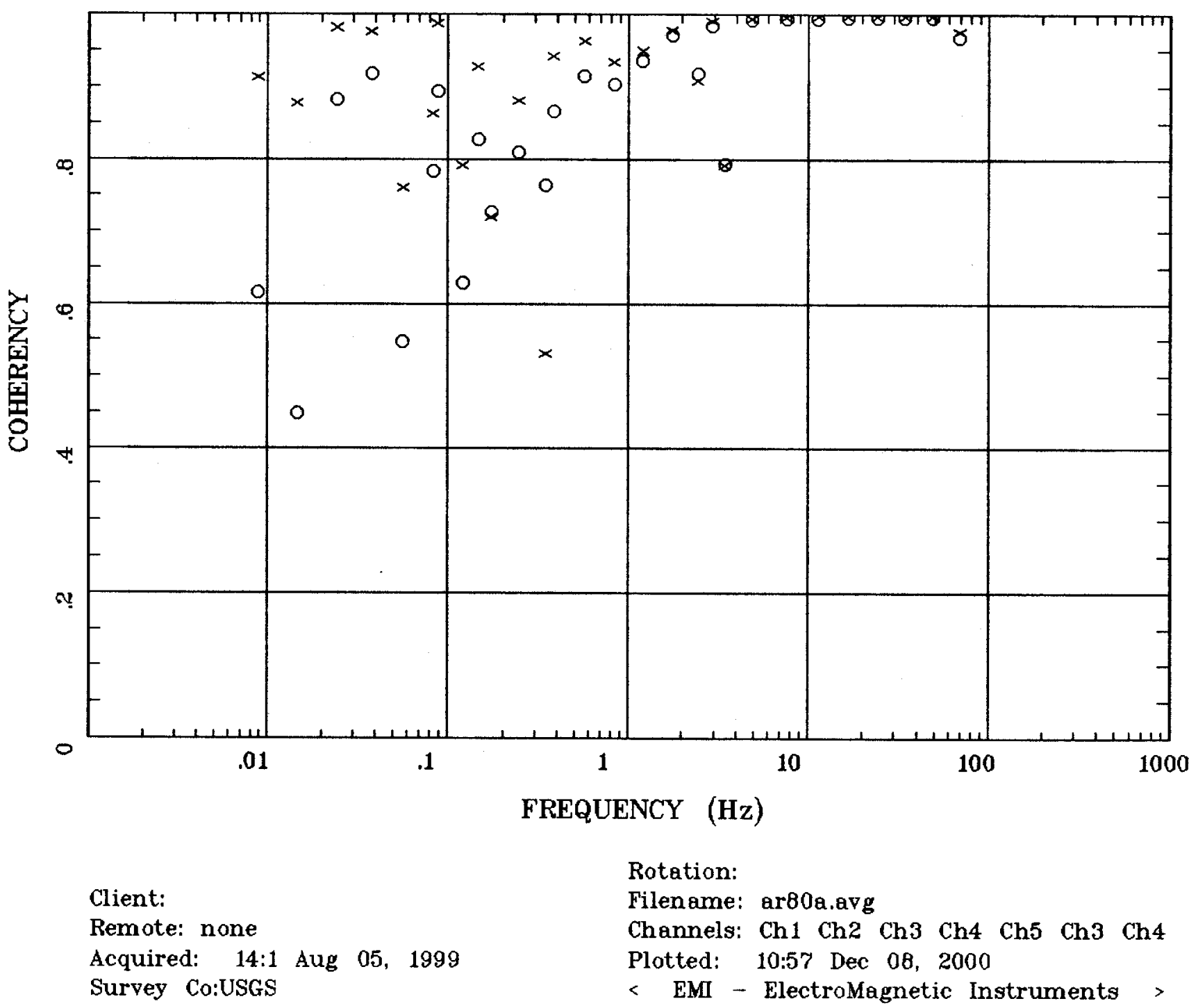


Station 80

POLAR PLOTS

Alligator Ridge

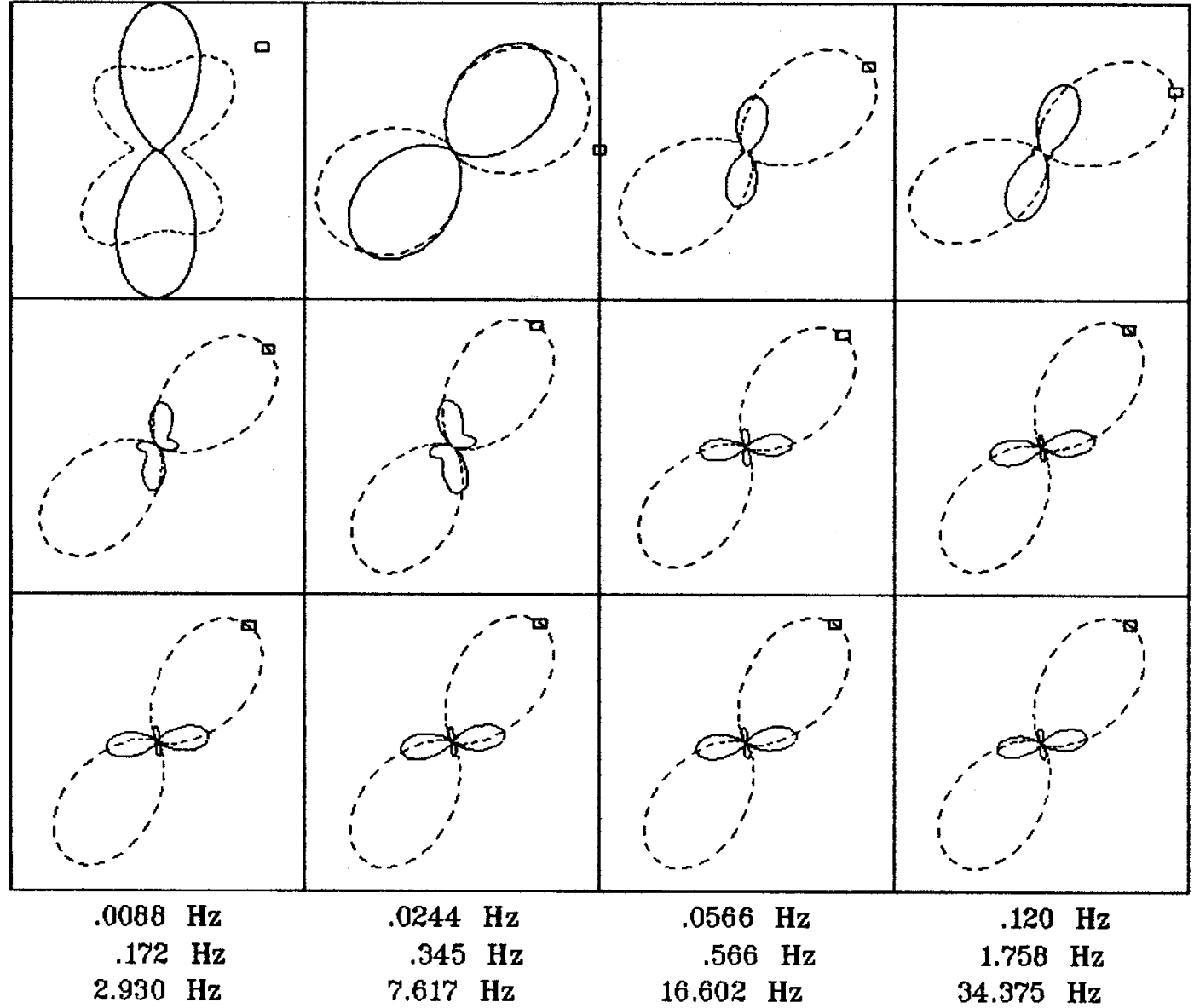

Client:

Remote: none

Acquired: 14:1 Aug 05, 1999

Survey Co:USGS

Rotation:

Filename: ar80a.avg

Channels: Ch1 Ch2 Ch3 Ch4 Ch5 Ch3 Ch4

Plotted: 10:57 Dec 08, 2000

< EMI - ElectroMagnetic Instruments > 


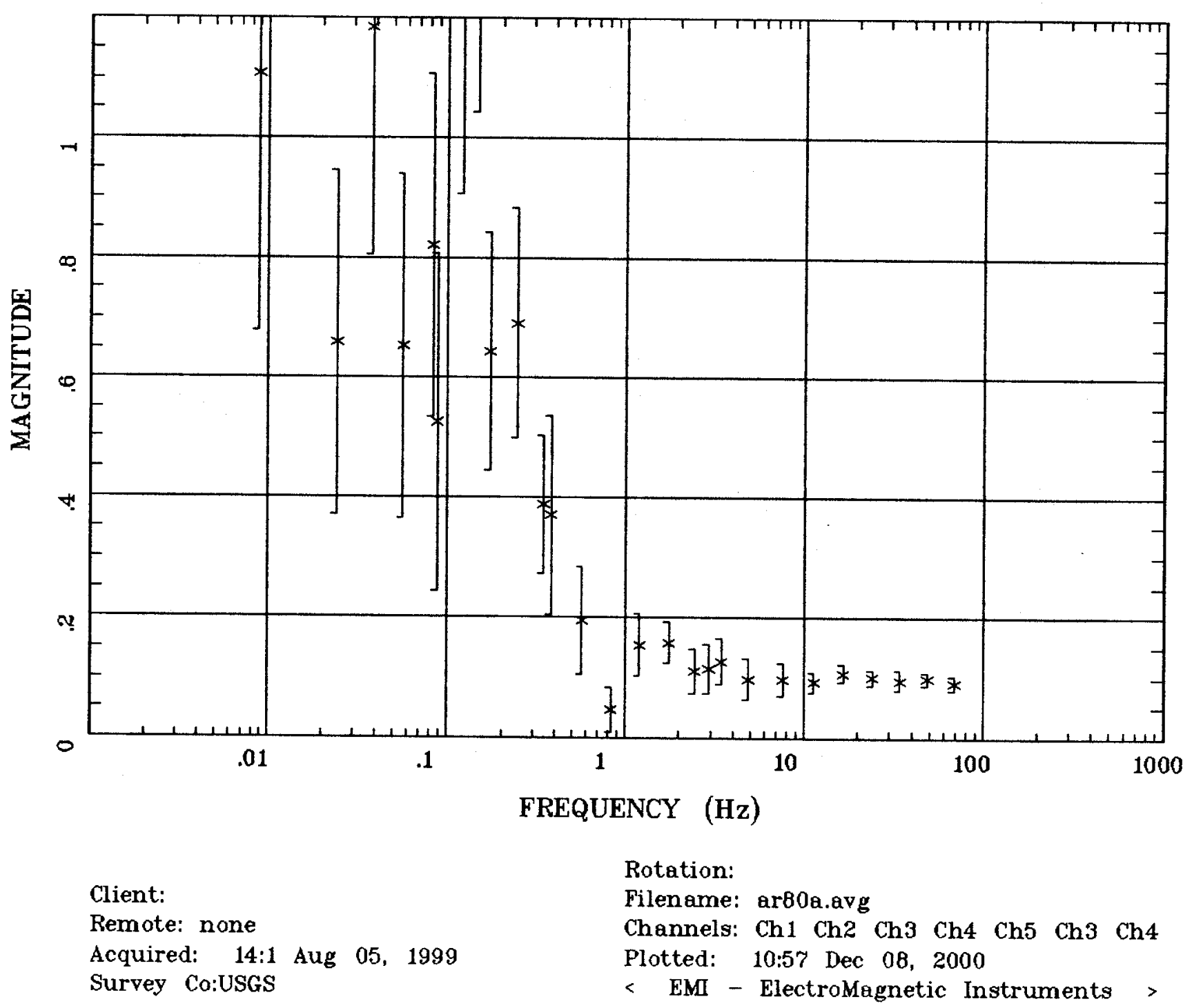




\section{Station 80}

\section{TIPPER STRIKE}

\section{Alligator Ridge}

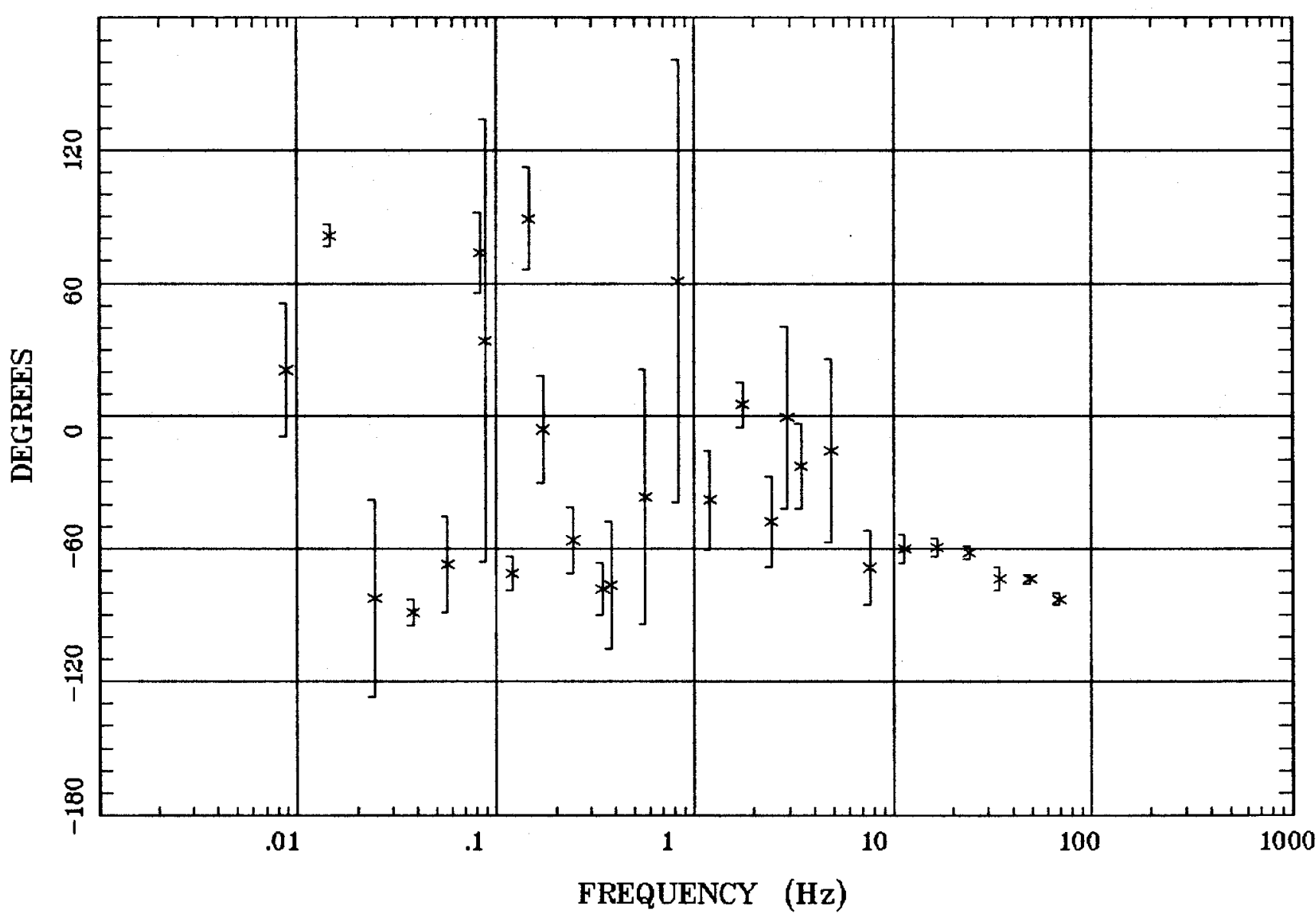

Client:

Remote: none

Acquired: 14:1 Aug 05, 1999 Survey Co:USGS
Rotation:

Filename: ar80a.avg

Channels: Ch1 Ch2 Ch3 Ch4 Ch5 Ch3 Ch4 Plotted: 10:57 Dec 08, 2000

< EMI - ElectroMagnetic Instruments 


\section{Station 80}

HzHx.x Coh HzHy.o

Alligator Ridge

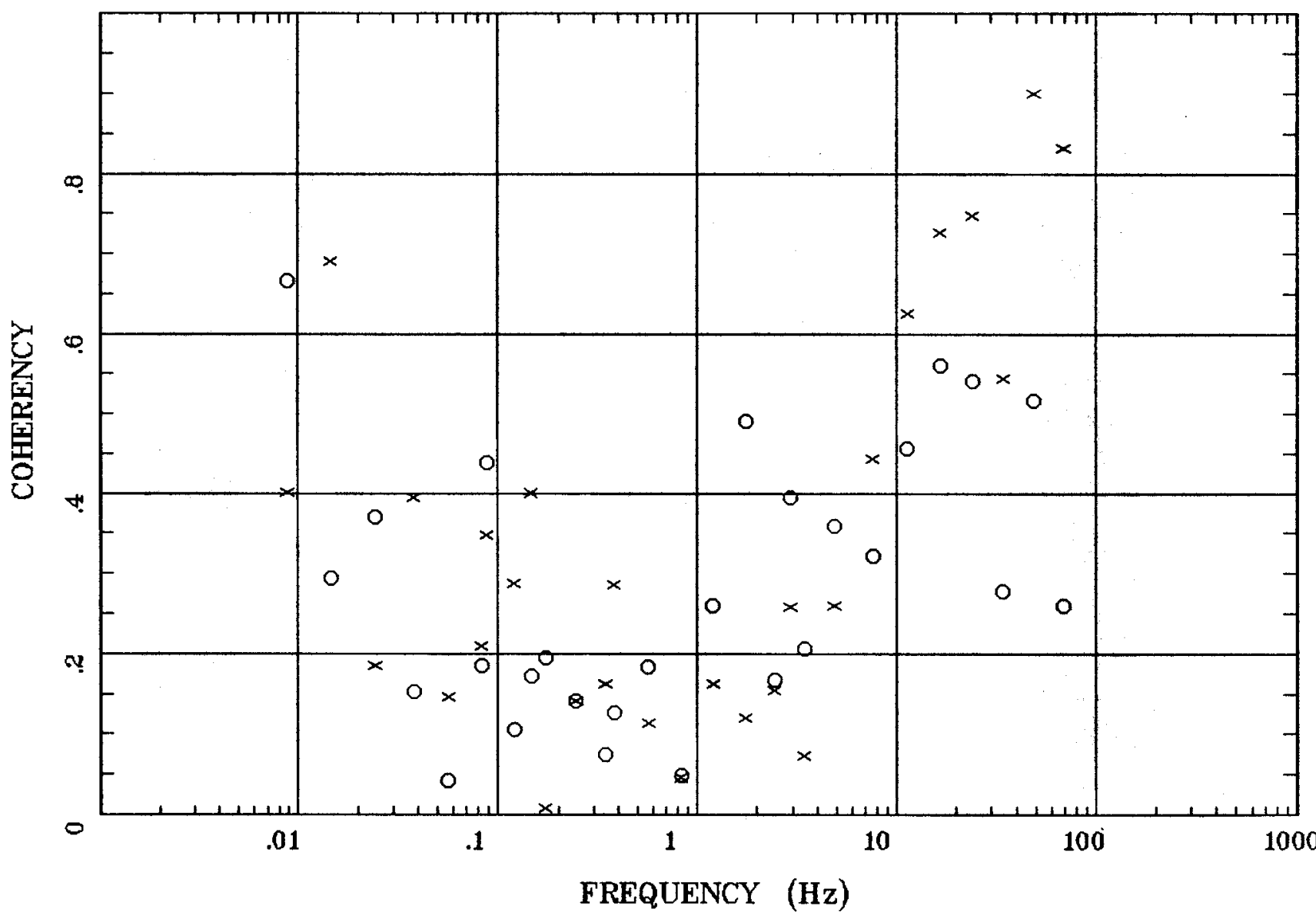

Client:

Remote: none

Acquired: 14:1 Aug 05, 1999 Survey Co:USGS
Rotation:

Filename: ar80a.avg

Channels: Ch1 Ch2 Ch3 Ch4 Ch5 Ch3 Ch4 Plotted: 10:57 Dec 08, 2000

$<$ EMI - ElectroMagnetic Instruments 


\section{Station 108}

\section{APPARENT RESISTIVITY}

Newark Lake, NV 100K

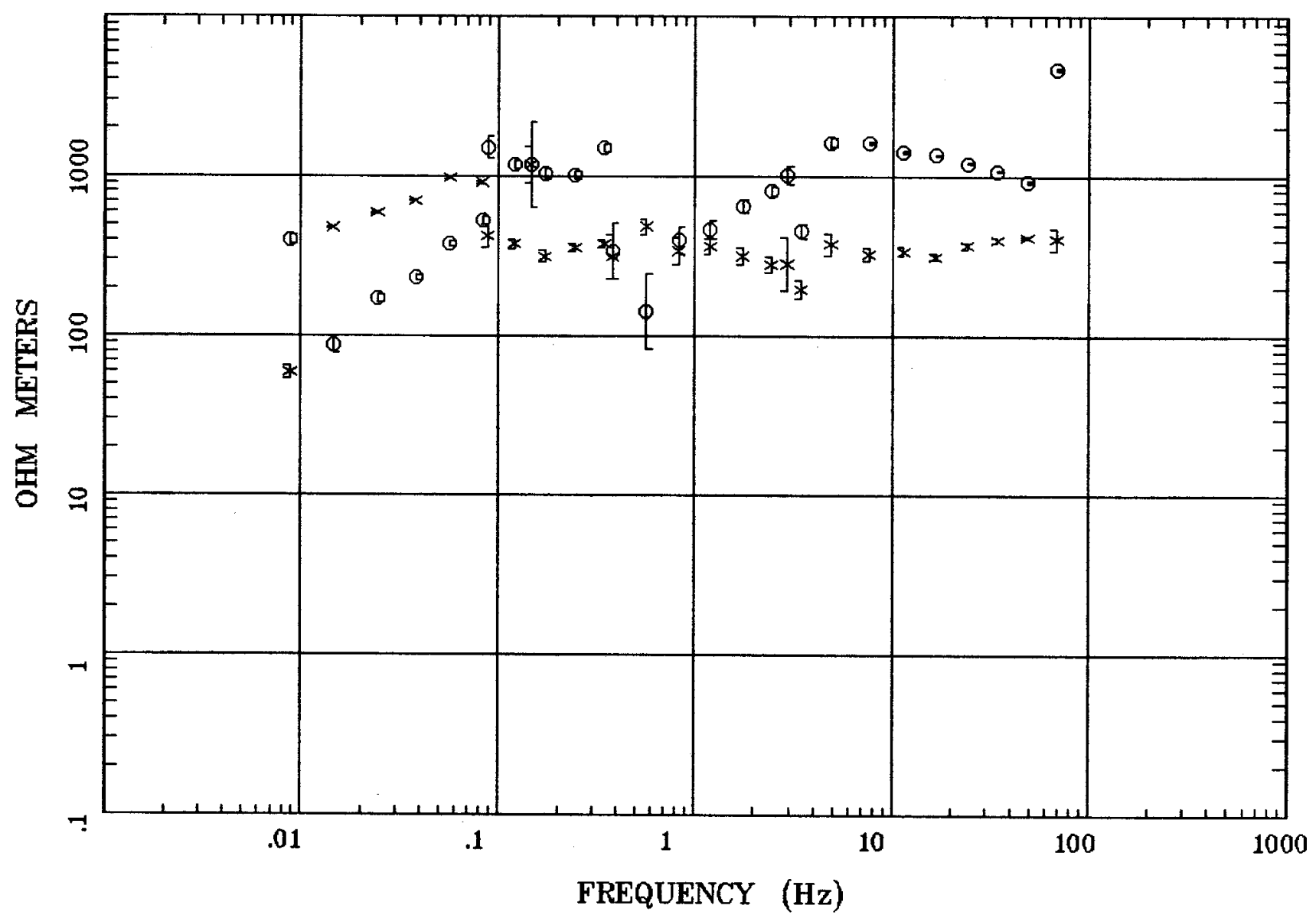

Client:

Remote: none

Acquired: 11:1 Aug 07, 2000

Survey Co:USGS
Rotation:

Filename: ar108b.avg

Channels: Ch1 Ch2 Ch3 Ch4 Ch5 Ch3 Ch4

Plotted: 10:51 Dec 08, 2000

< EMI - ElectroMagnetic Instruments > 


\section{Station 108}

IMPEDANCE PHASE

Newark Lake, NV 100K

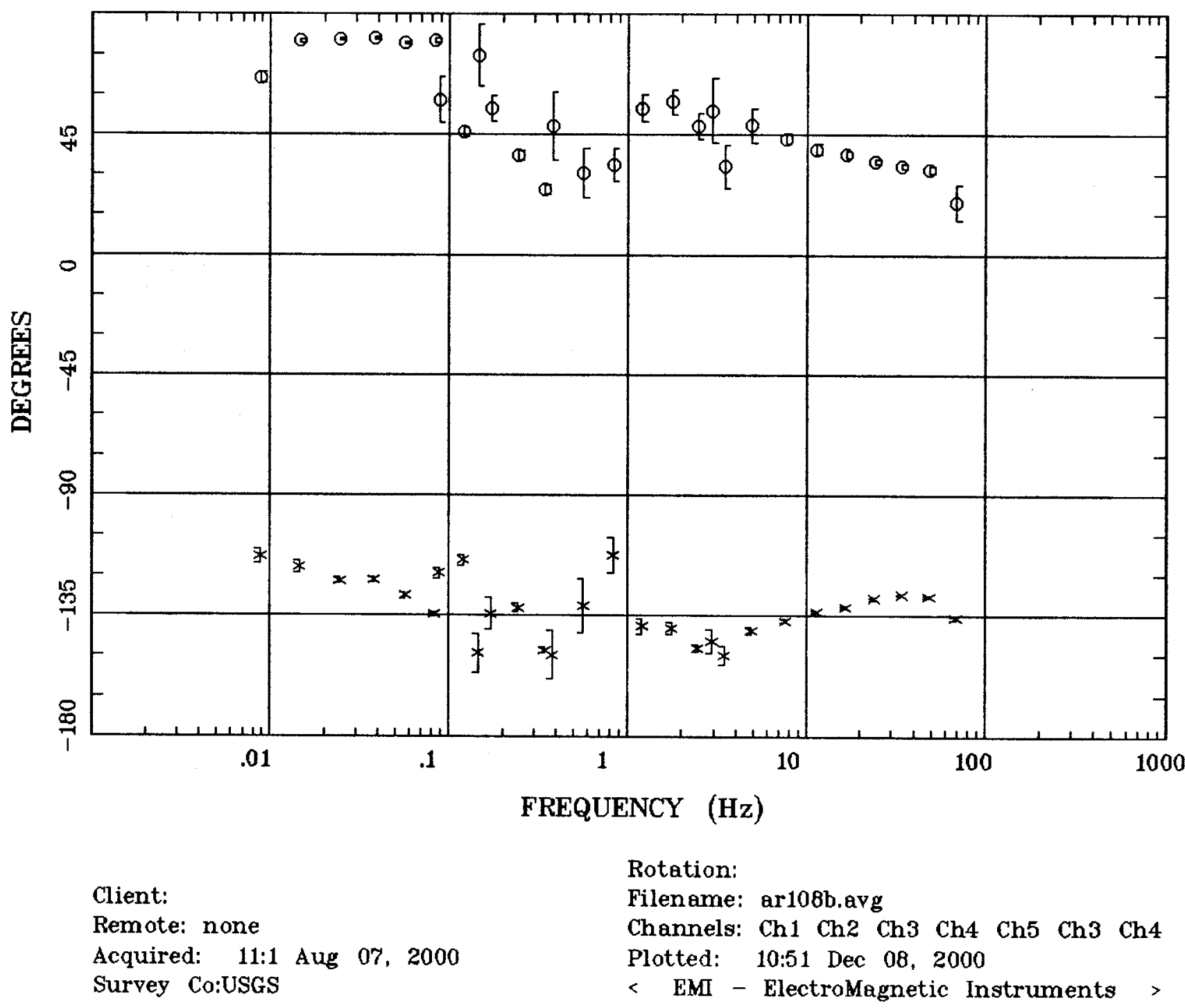


Station 108

ROTATION ANGLE

New ark Lake, NV 100K

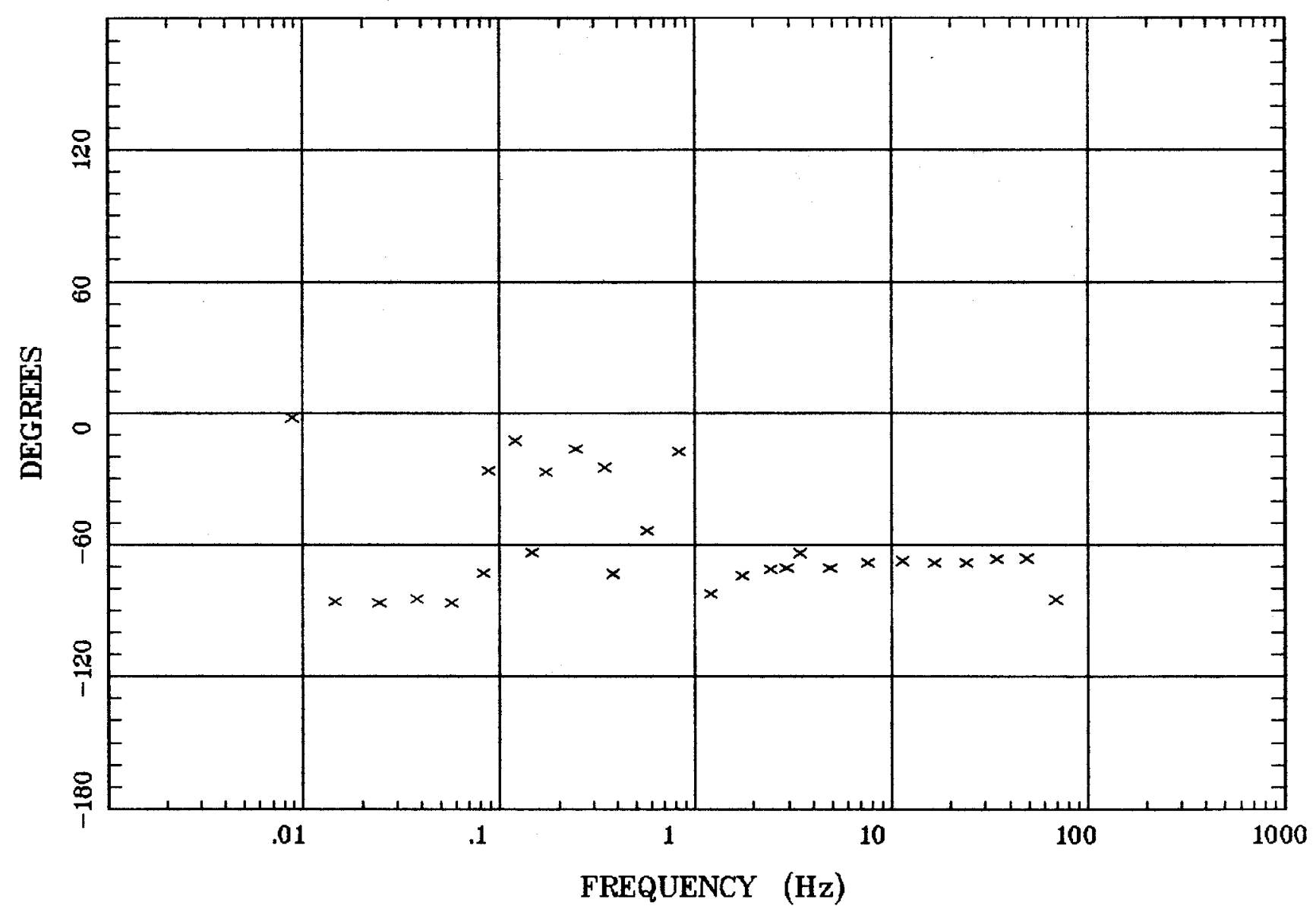

Client:

Remote: none

Acquired: 11:1 Aug 07, 2000

Survey Co:USGS
Rotation:

Filename: ar108b.avg

Channels: Ch1 Ch2 Ch3 Ch4 Ch5 Ch3 Ch4

Plotted: 10:51 Dec 08, 2000

< EMI - ElectroMagnetic Instruments > 
Station 108

IMPEDANCE SKEW

Newark Lake, NV 100K

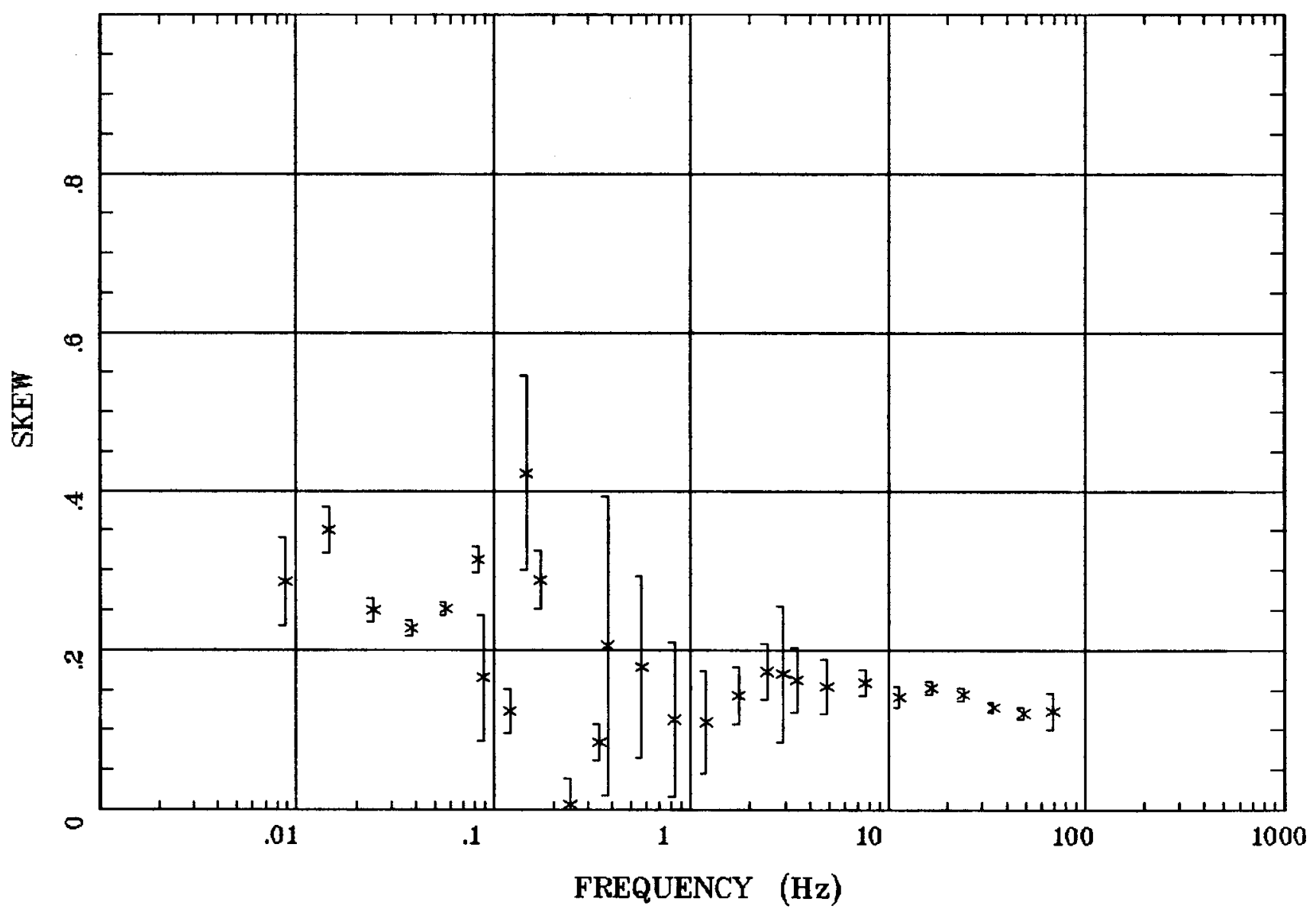

Client:

Remote: none

Rotation:

Filename: ar108b.avg

Channels: Ch1 Ch2 Ch3 Ch4 Ch5 Ch3 Ch4

Acquired: 11:1 Aug 07, 2000

Survey Co:USGS

Plotted: 10:51 Dec 08, 2000

$<$ EMI - ElectroMagnetic Instruments > 


\section{Station 108}

E MULT Coh.

Newark Lake, NV 100K

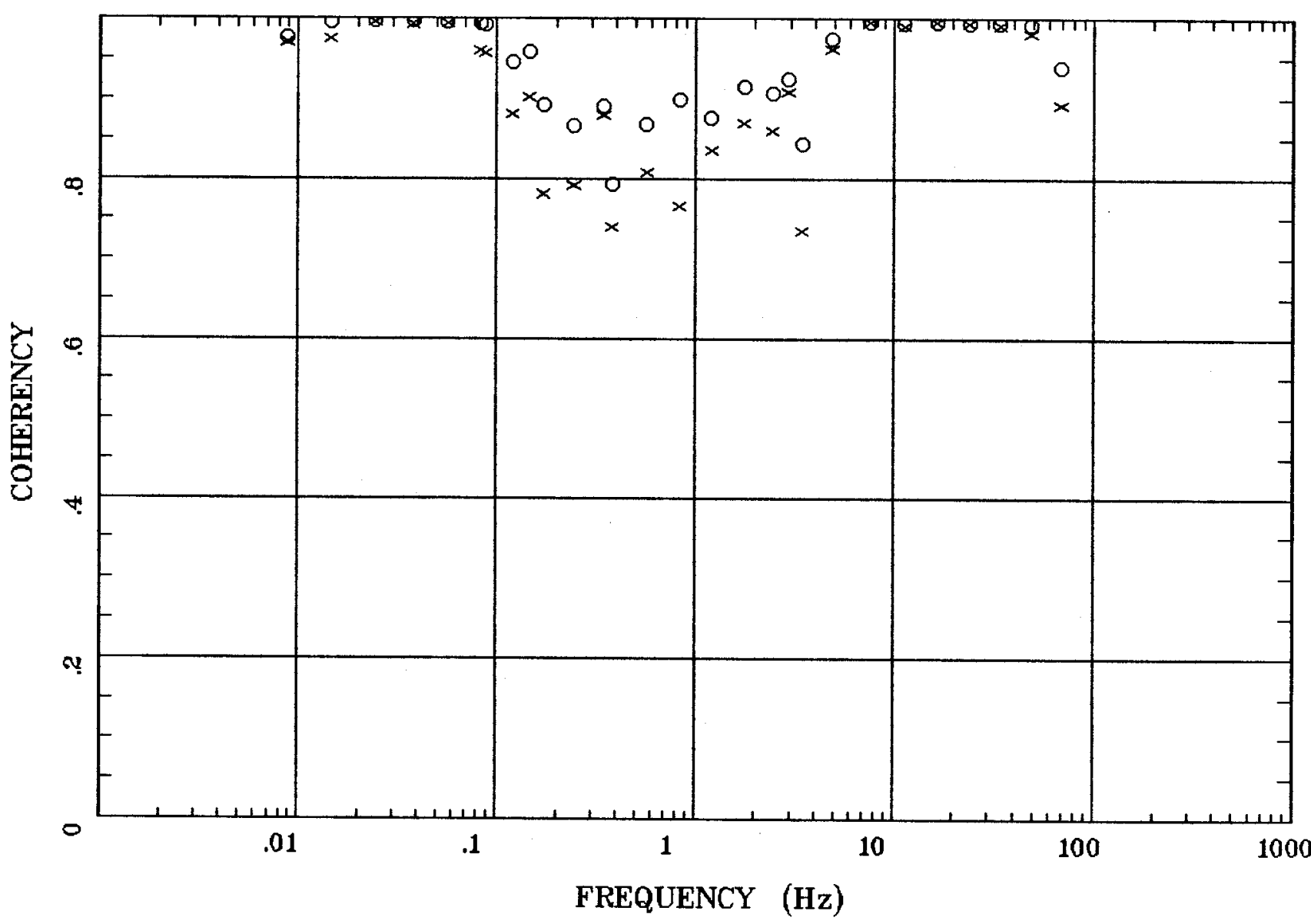

Client:

Remote: none

Acquired: 11:1 Aug 07, 2000

Survey Co:USGS
Rotation:

Filename: ar108b.avg

Channels: Ch1 Ch2 Ch3 Ch4 Ch5 Ch3 Ch4 Plotted: 10:51 Dec 08, 2000

< EMI - ElectroMagnetic Instruments > 


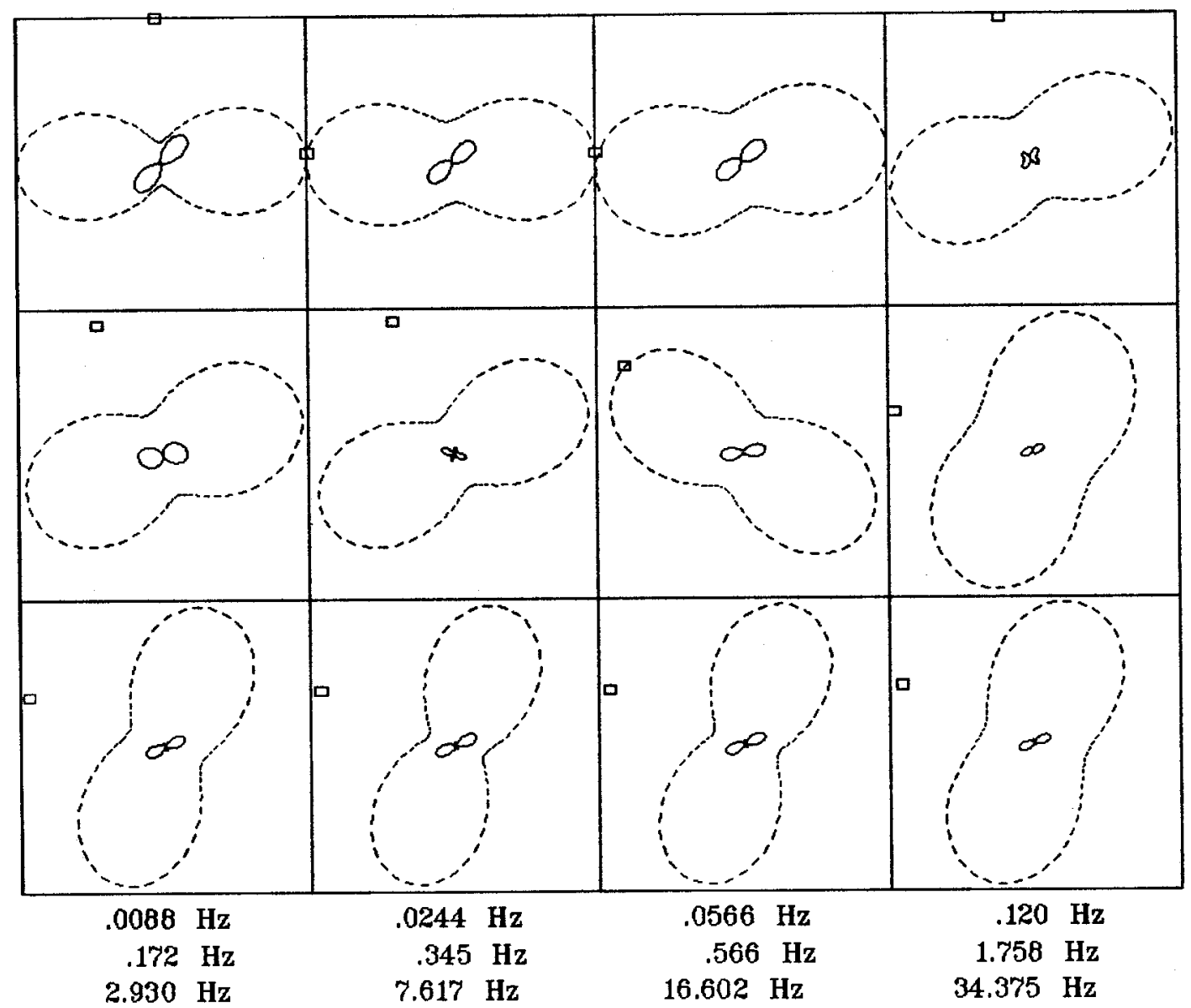

Client:

Remote: none

Acquired: 11:1 Aug 07, 2000 Survey Co:USGS

\section{Rotation:}

Filename: ar108b.avg

Channels: Ch1 Ch2 Ch3 Ch4 Ch5 Ch3 Ch4 Plotted: 10:51 Dec 08, 2000

< EMI - ElectroMagnetic Instruments > 


\section{TIPPER MAGNITUDE}

Newark Lake, NV 100K

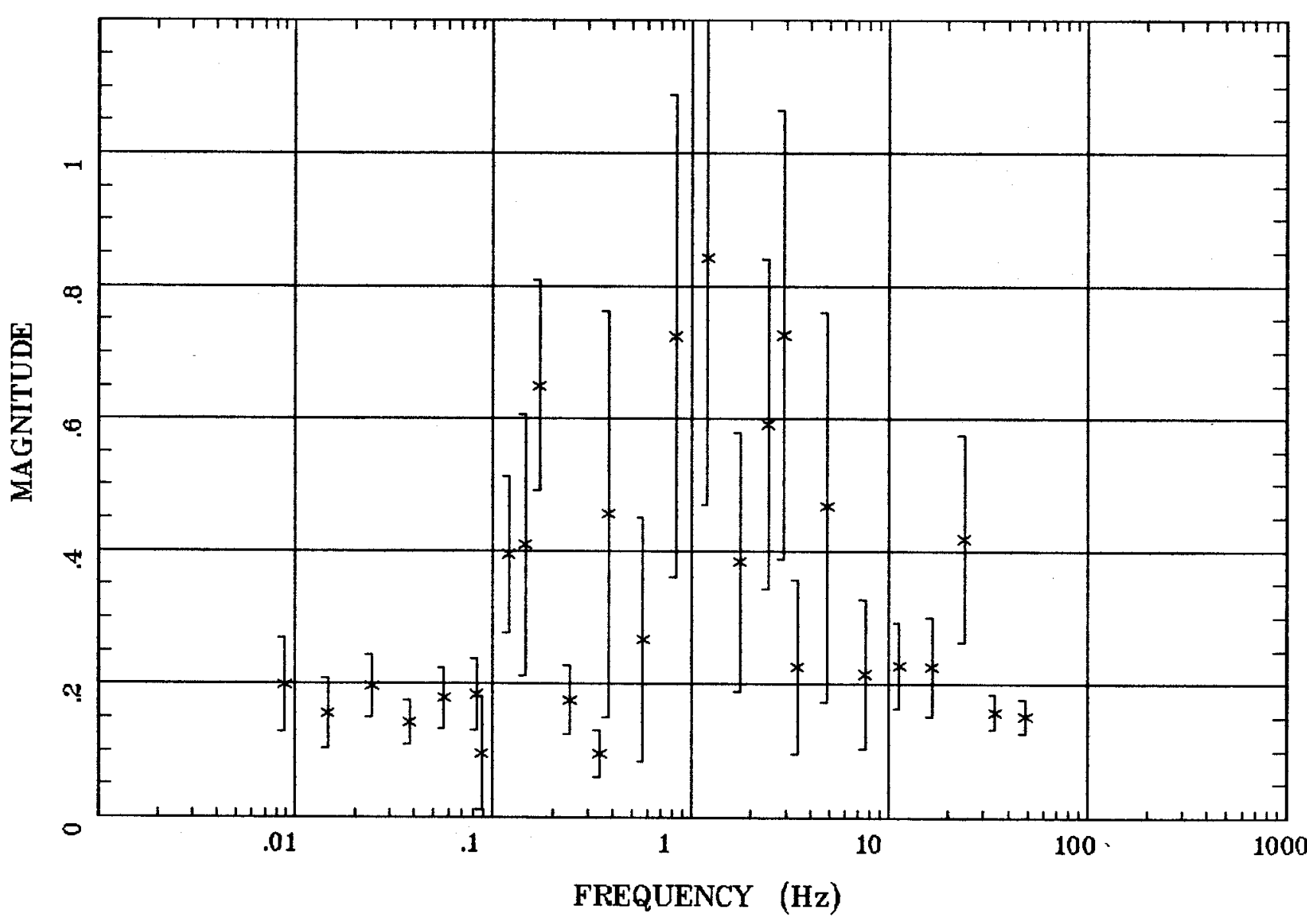

Client:

Remote: none

Acquired: 11:1 Aug 07, 2000

Survey Co:USGS
Rotation:

Filename: ar108b.avg

Channels: Ch1 Ch2 Ch3 Ch4 Ch5 Ch3 Ch4 Plotted: 10:52 Dec 08, 2000

$<$ EMI - ElectroMagnetic Instruments 
Station 108

TIPPER STRIKE

Newark Lake, NV 100K

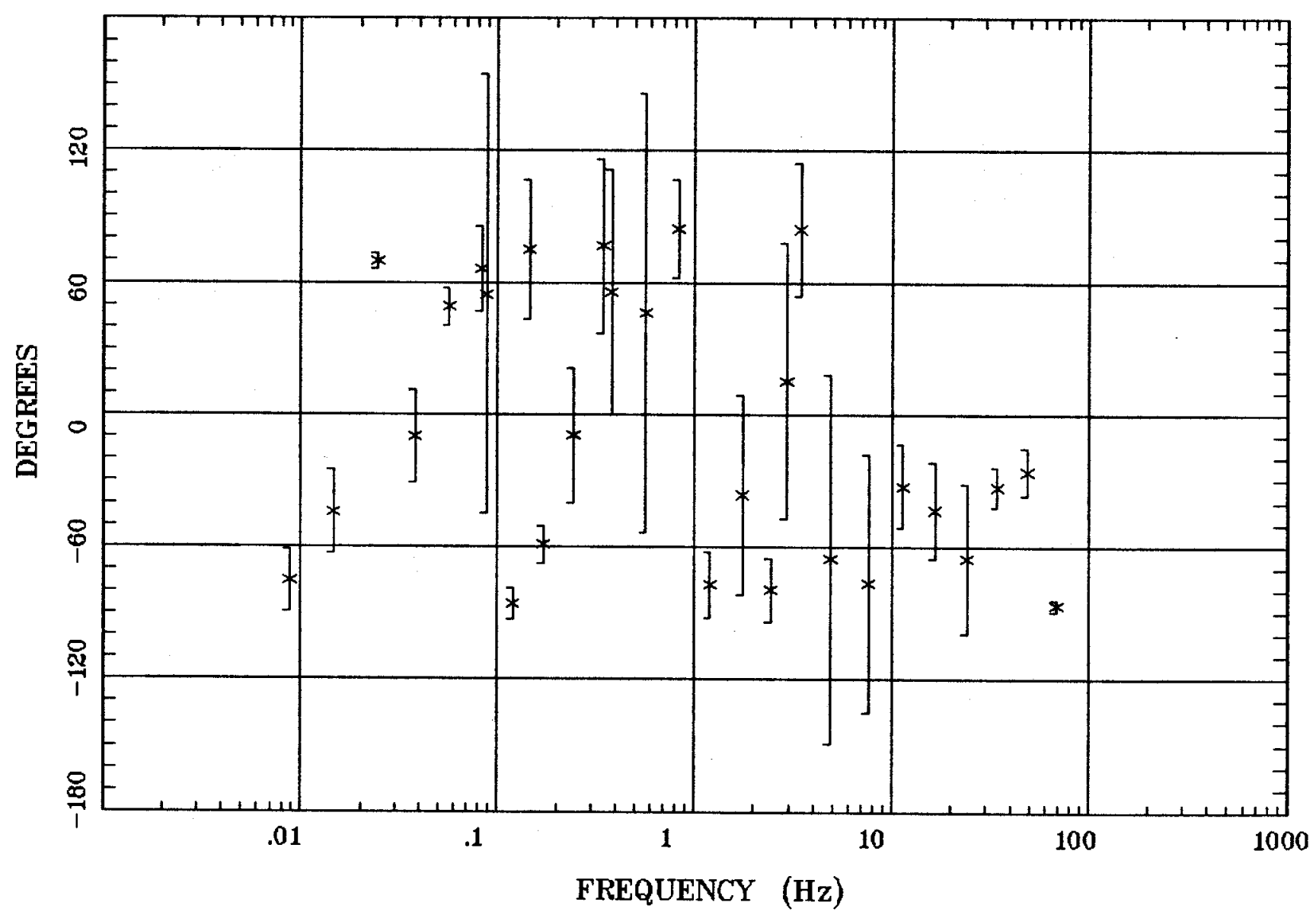

Client:

Remote: none

Acquired: 11:1 Aug 07, 2000

Survey Ca:USGS

Rotation:

Filename: ar108b.avg

Channels: Ch1 Ch2 Ch3 Ch4 Ch5 Ch3 Ch4

Plotted: 10:52 Dec 08, 2000

< EMI - ElectroMagnetic Instruments > 
HzHx.x Coh HzHy.o

Newark Lake, NV 100K

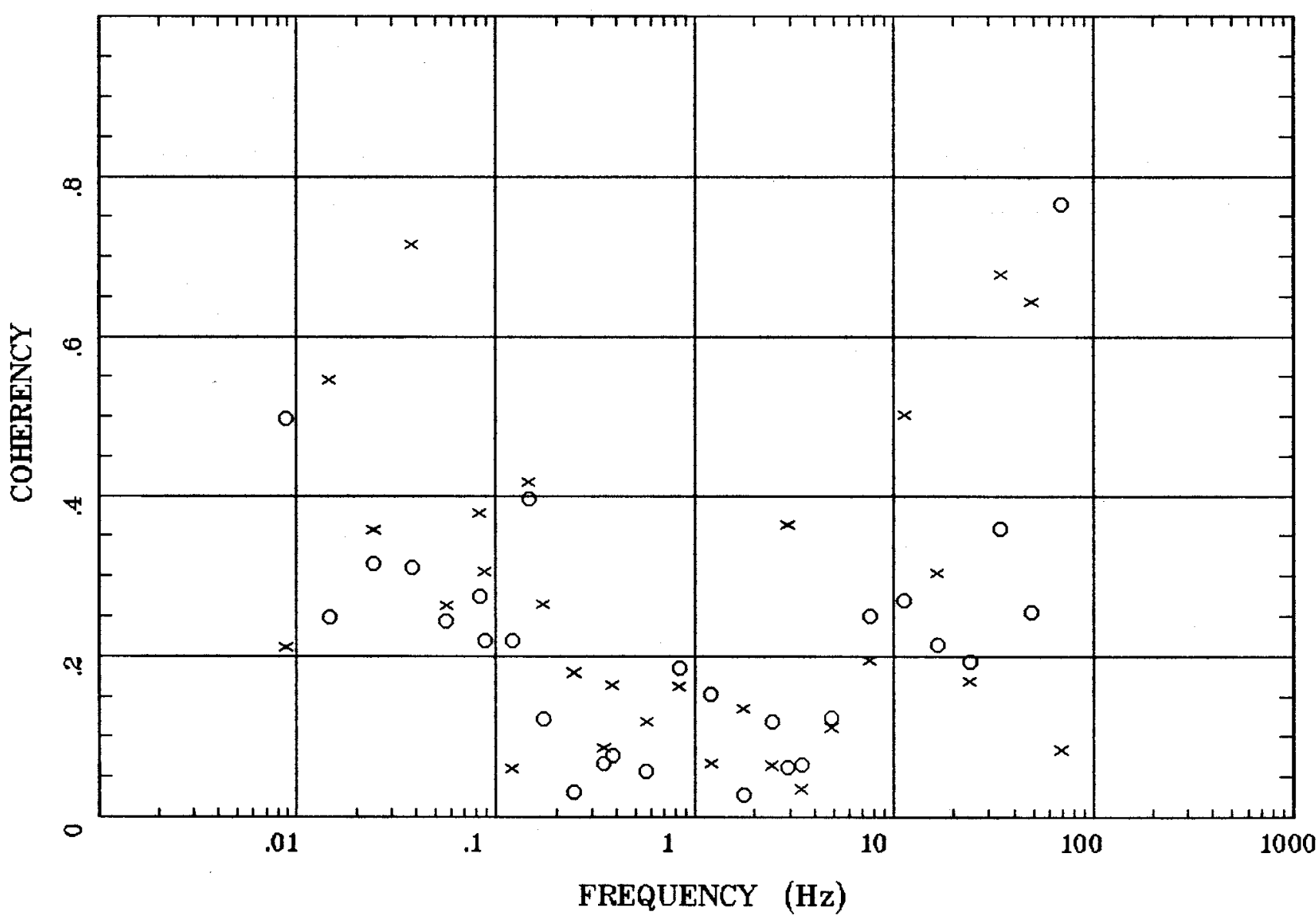

Client:

Remote: none

Acquired: 11:1 Aug 07, 2000 Survey Co:USGS
Rotation:

Filename: ar108b.avg

Channels: Ch1 Ch2 Ch3 Ch4 Ch5 Ch3 Ch4 Plotted: 10:52 Dec 08, 2000

< EMI - ElectroMagnetic Instruments > 
Station 79

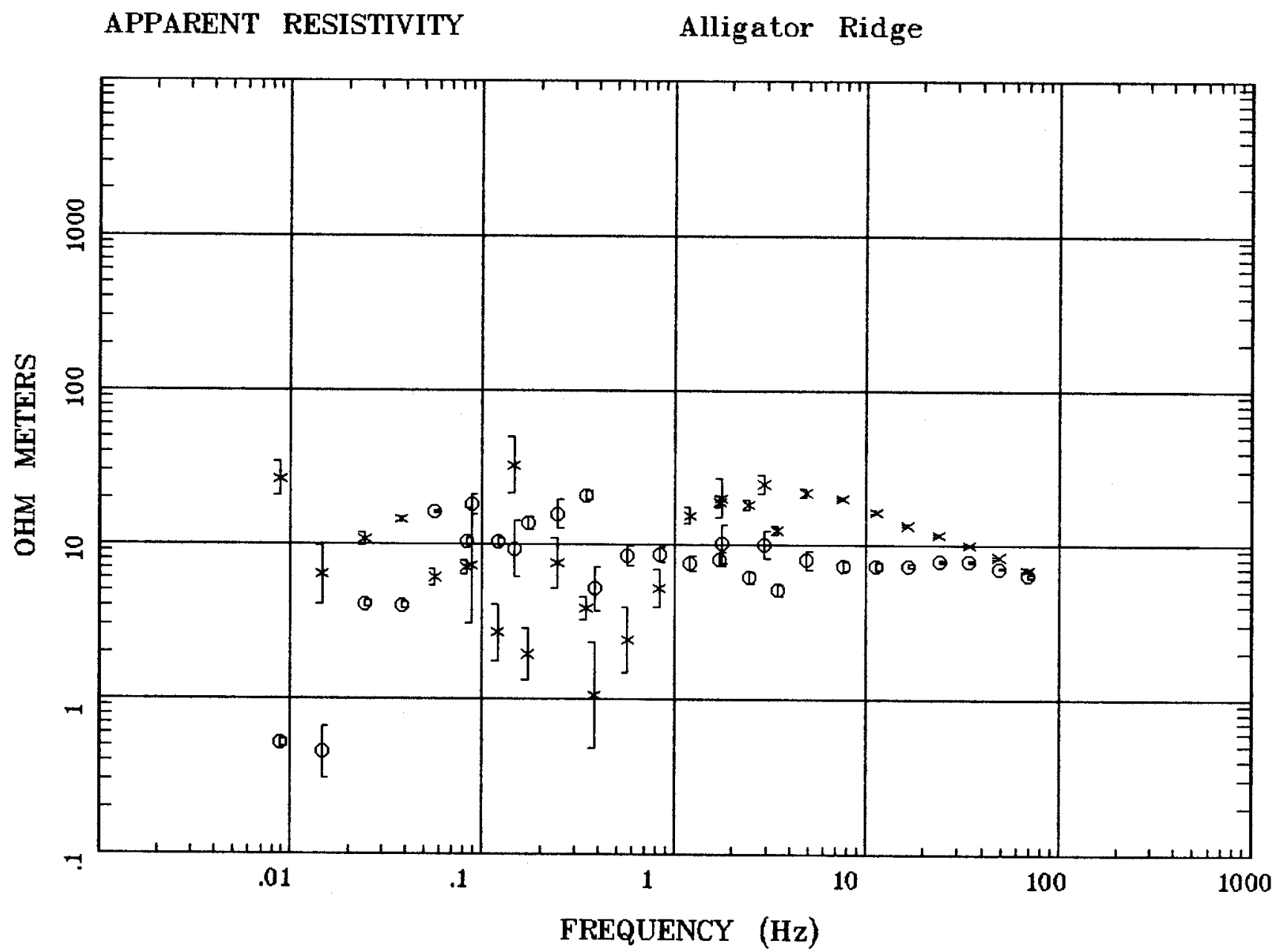

Client:

Remote: none

Acquired: 10:4 Aug 05, 1999

Survey Co:USGS
Rotation:

Filename: ar79.avg

Channels: Ch1 Ch2 Ch3 Ch4 Ch5 Ch3 Ch4

Plotted: 10:56 Dec 08, 2000

< EMI - ElectroMagnetic Instruments > 


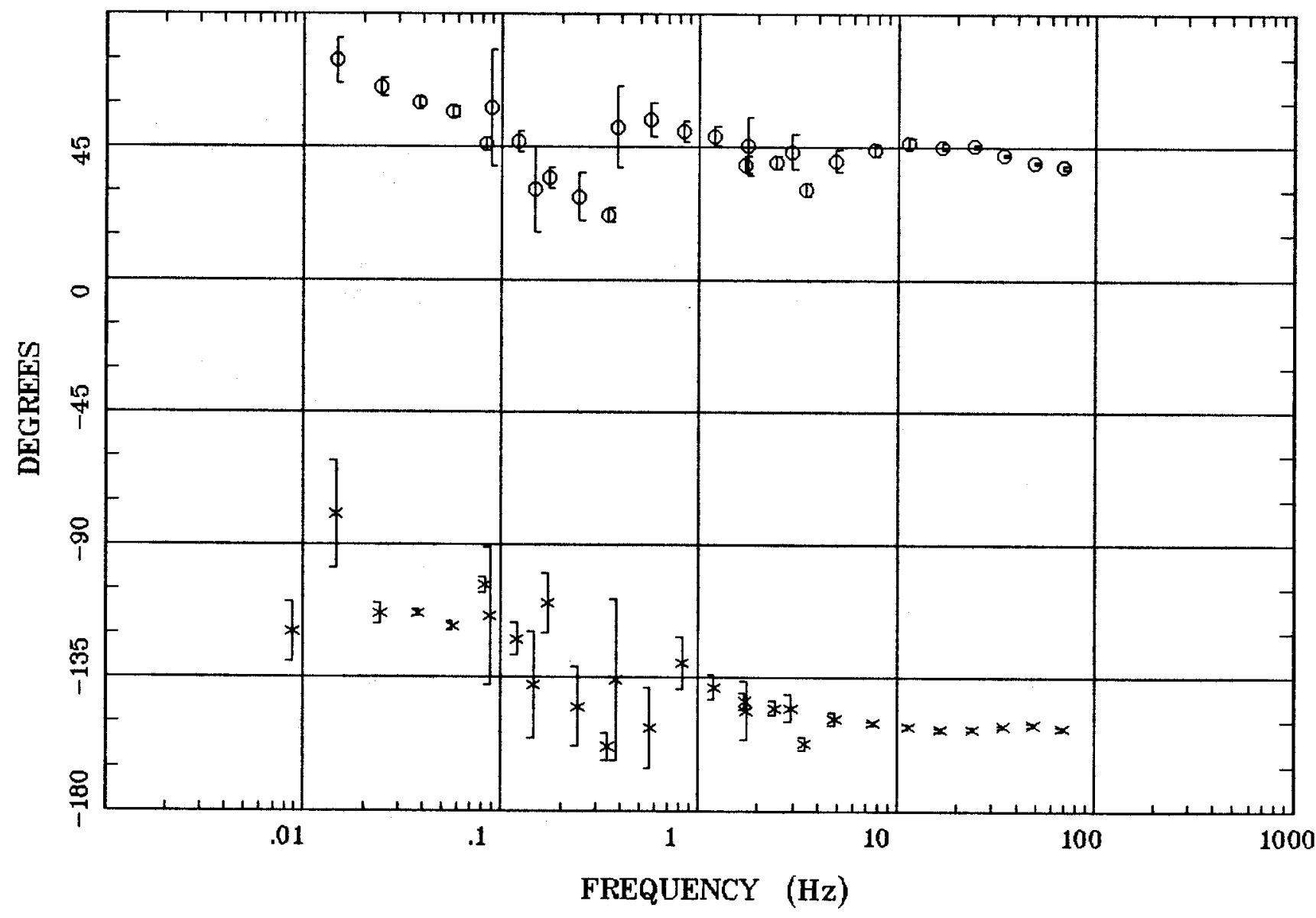

Client:

Remote: none

Acquired: 10:4 Aug 05, 1999 Survey Co:USGS
Rotation:

Filename: ar79.avg

Channels: Ch1 Ch2 Ch3 Ch4 Ch5 Ch3 Ch4

Plotted: 10:56 Dec 08, 2000

$<$ EMI - ElectroMagnetic Instruments 


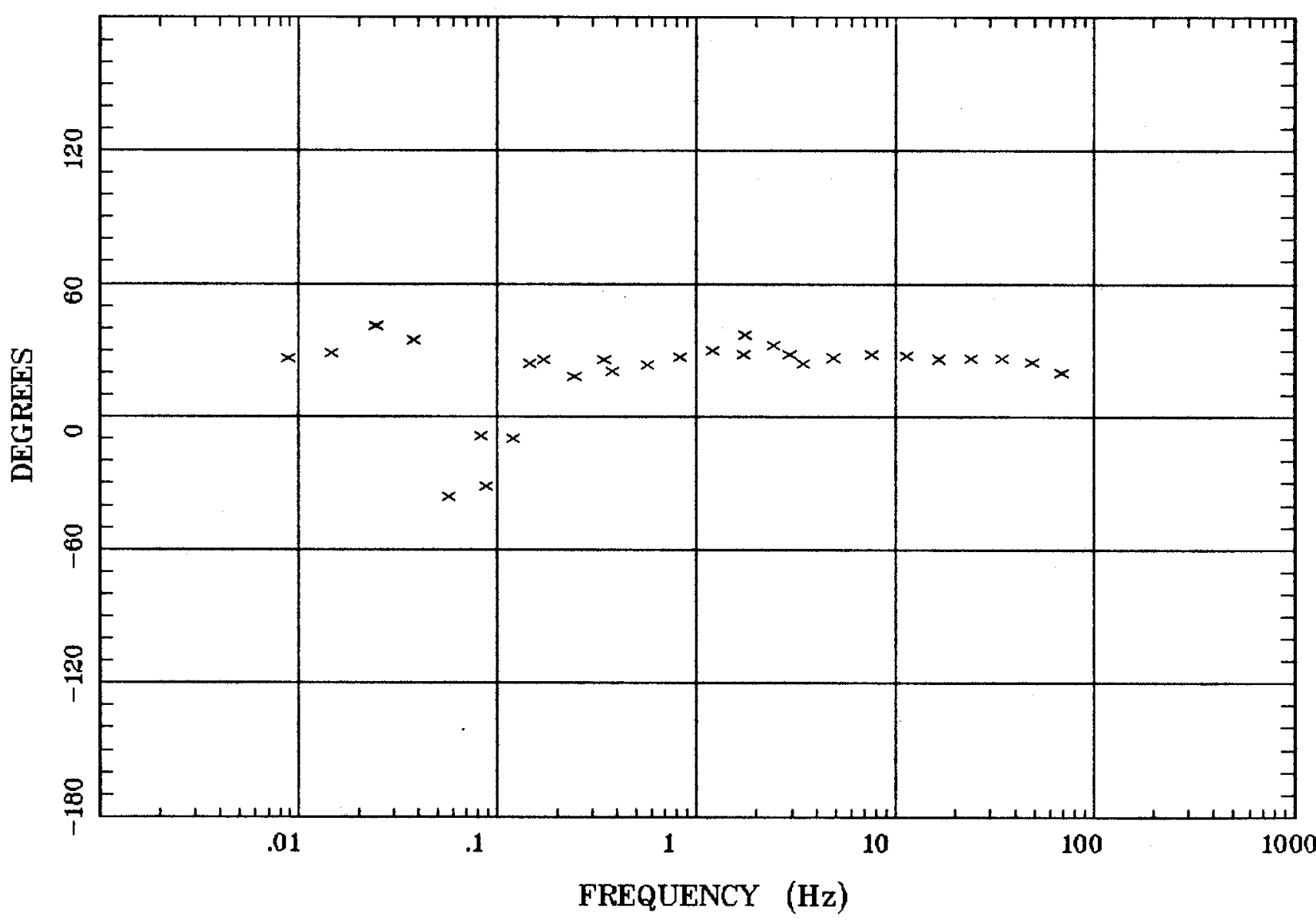

Client:

Remote: none

Acquired: 10:4 Aug 05, 1999 Survey Co:USGS
Rotation:

Filename: ar79.avg

Channels: Ch1 Ch2 Ch3 Ch4 Ch5 Ch3 Ch4 Plotted: 10:56 Dec 08, 2000

< EMI - ElectroMagnetic Instruments 


\section{Station 79}

IMPEDANCE SKEW

Alligator Ridge

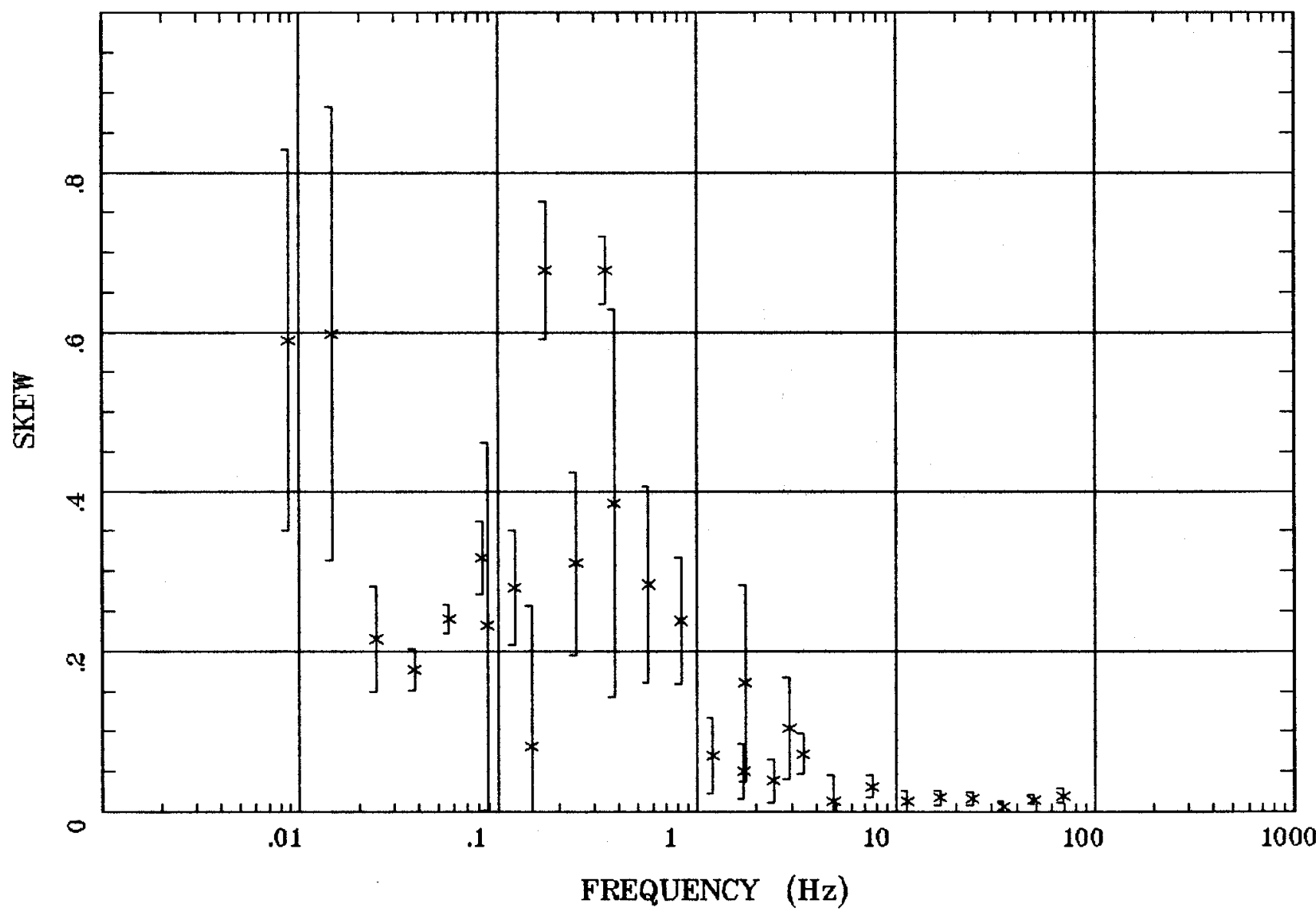

Client:

Remote: none

Acquired: 10:4 Aug 05, 1999 Survey Co:USGS
Rotation:

Fileneme: ar79.avg

Channels: Ch1 Ch2 Ch3 Ch4 Ch5 Ch3 Ch4 Plotted: 10:56 Dec 08, 2000

$<$ EMI - ElectroMagnetic Instruments 
Station 79

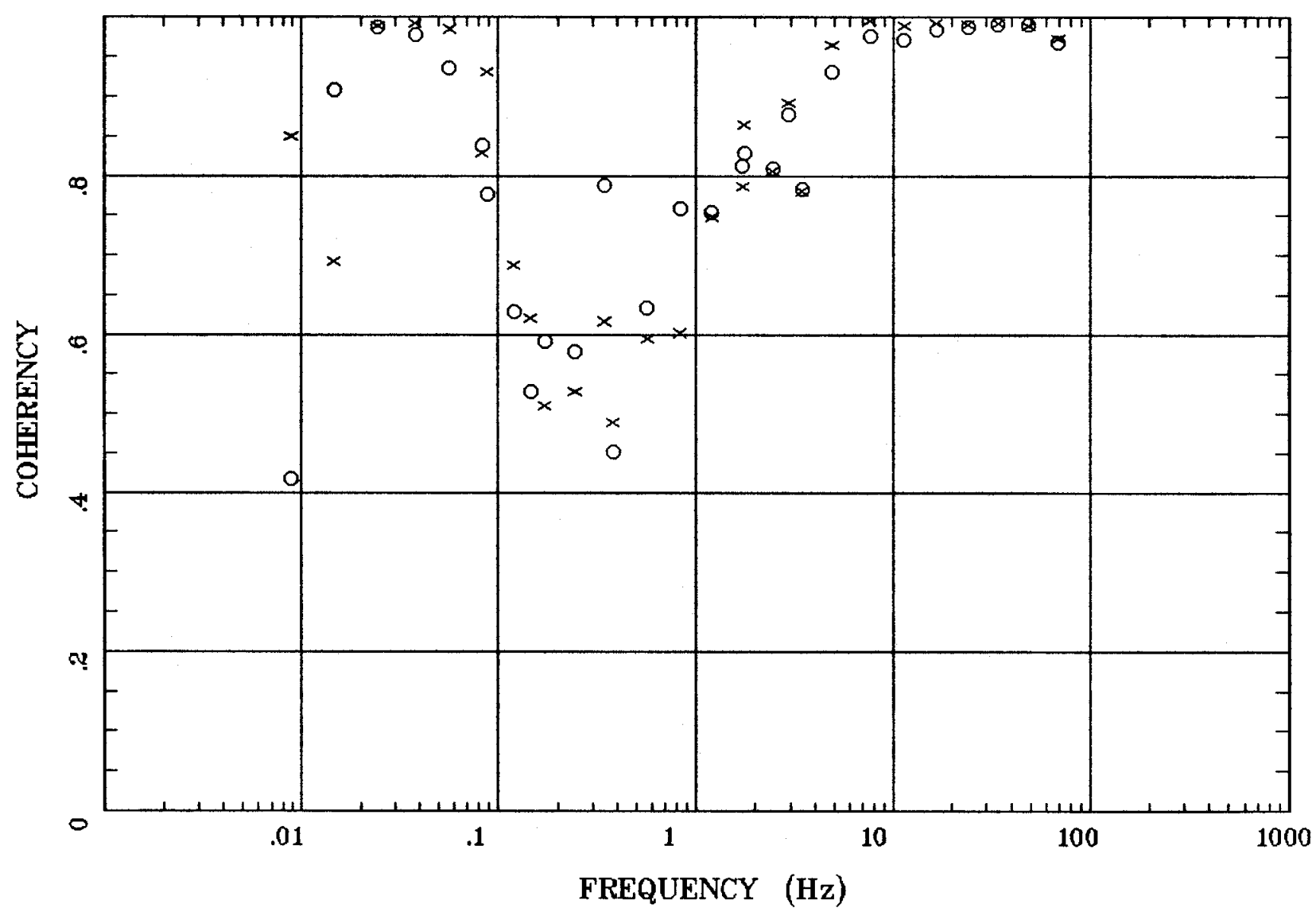

Client:

Remote: none

Acquired: 10:4 Aug 05, 1999

Survey Co:USGS
Rotation:

Filename: ar79.avg

Channels: Ch1 Ch2 Ch3 Ch4 Ch5 Ch3 Ch4

Platted: 10:56 Dec 08, 2000

< EMI - ElectroMagnetic Instruments > 


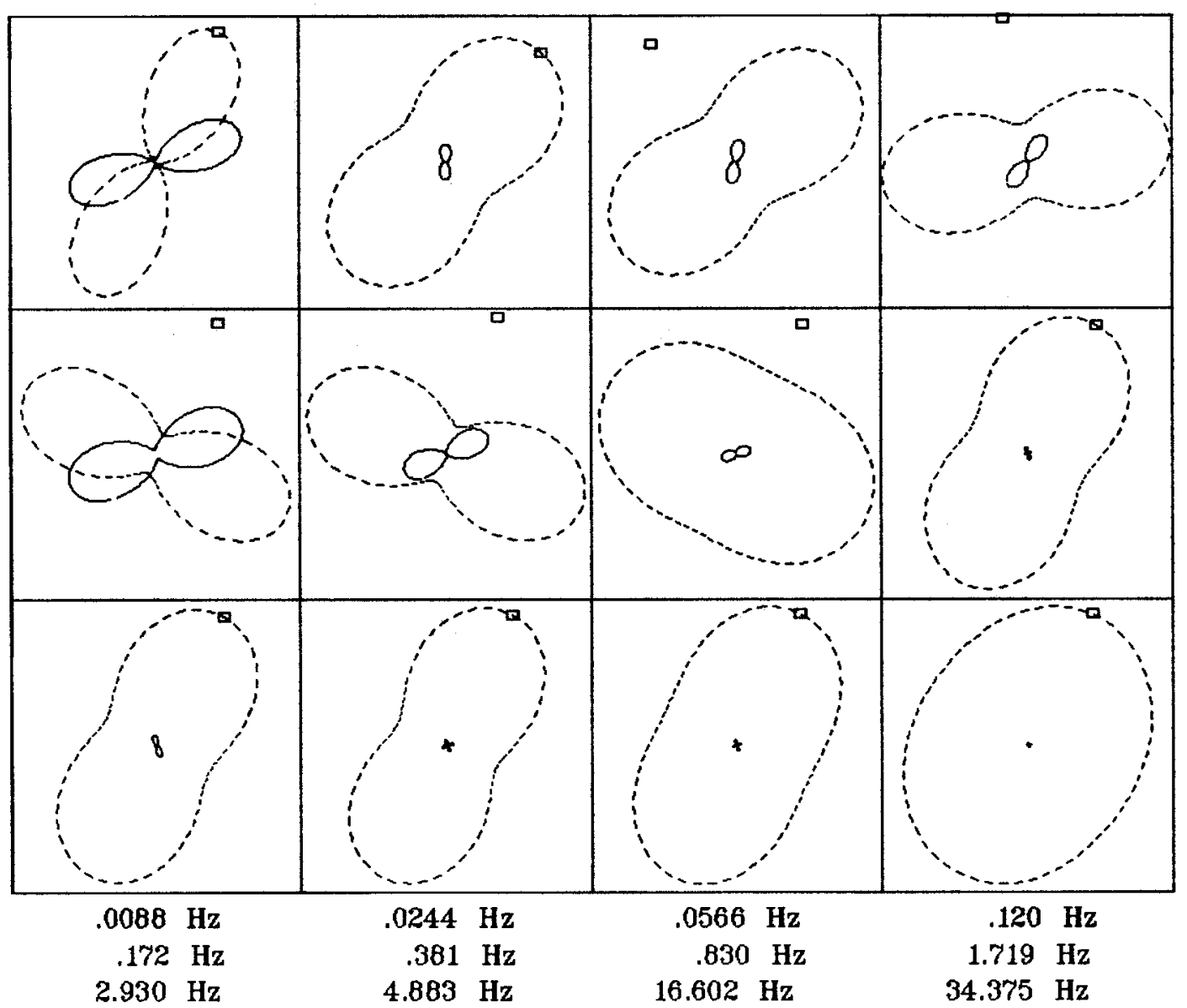

Client:

Remote: none

Acquired: 10:4 Aug 05, 1999 Survey Co:USGS
Rotation:

Filename: ar79.avg

Channels: Ch1 Ch2 Ch3 Ch4 Ch5 Ch3 Ch4 Plotted: 10:56 Dec 08, 2000

< EMI - ElectroMagnetic Instruments 


\section{Station 79}

\section{TIPPER MAGNITUDE}

Alligator Ridge

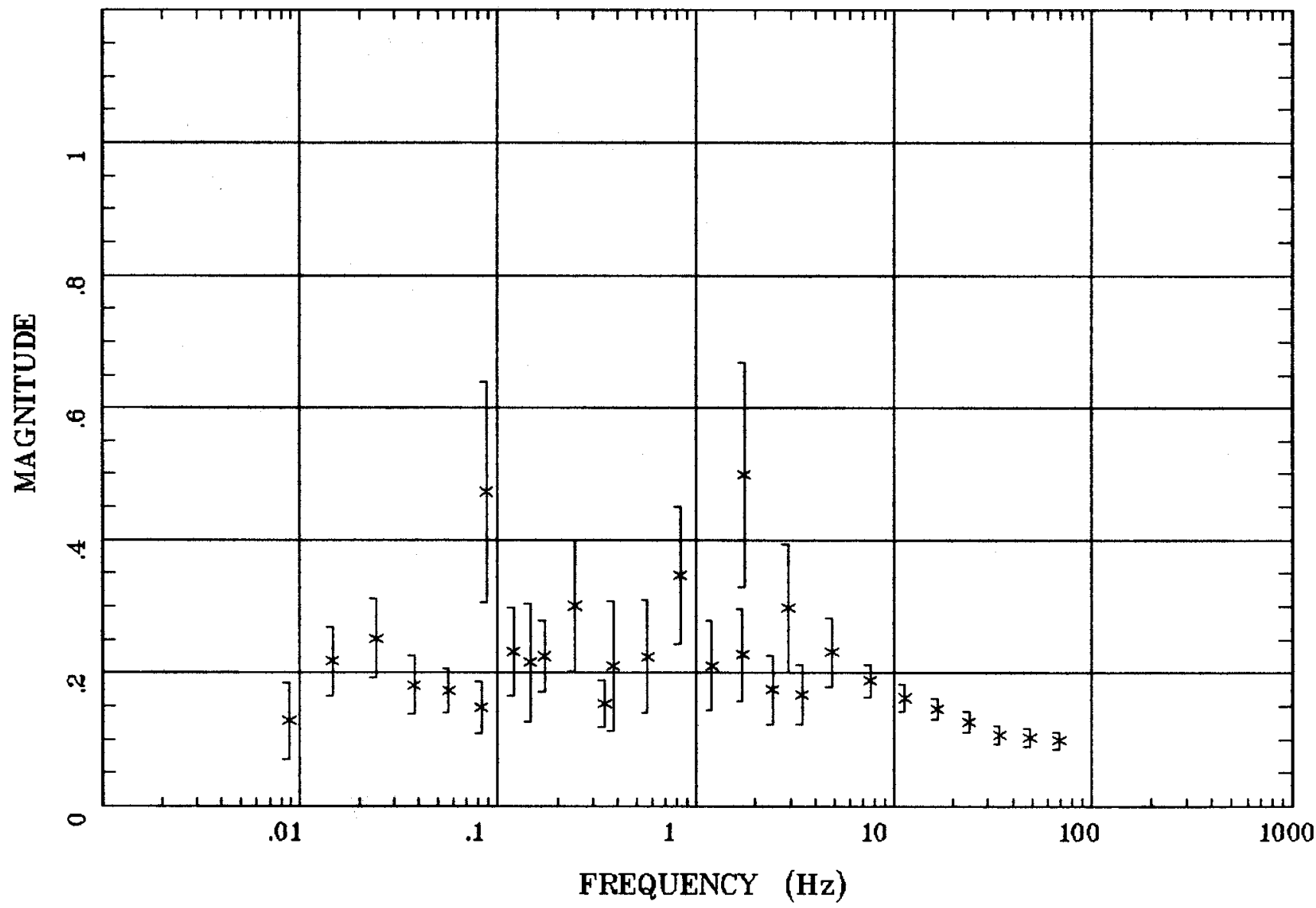

Client:

Remote: none

Acquired: 10:4 Aug 05, 1999

Survey Co:USGS
Rotation:

Filename: ar79.avg

Channels: Ch1 Ch2 Ch3 Ch4 Ch5 Ch3 Ch4

Plotted: 10:56 Dec 08, 2000

$<$ EMI - ElectroMagnetic Instruments > 


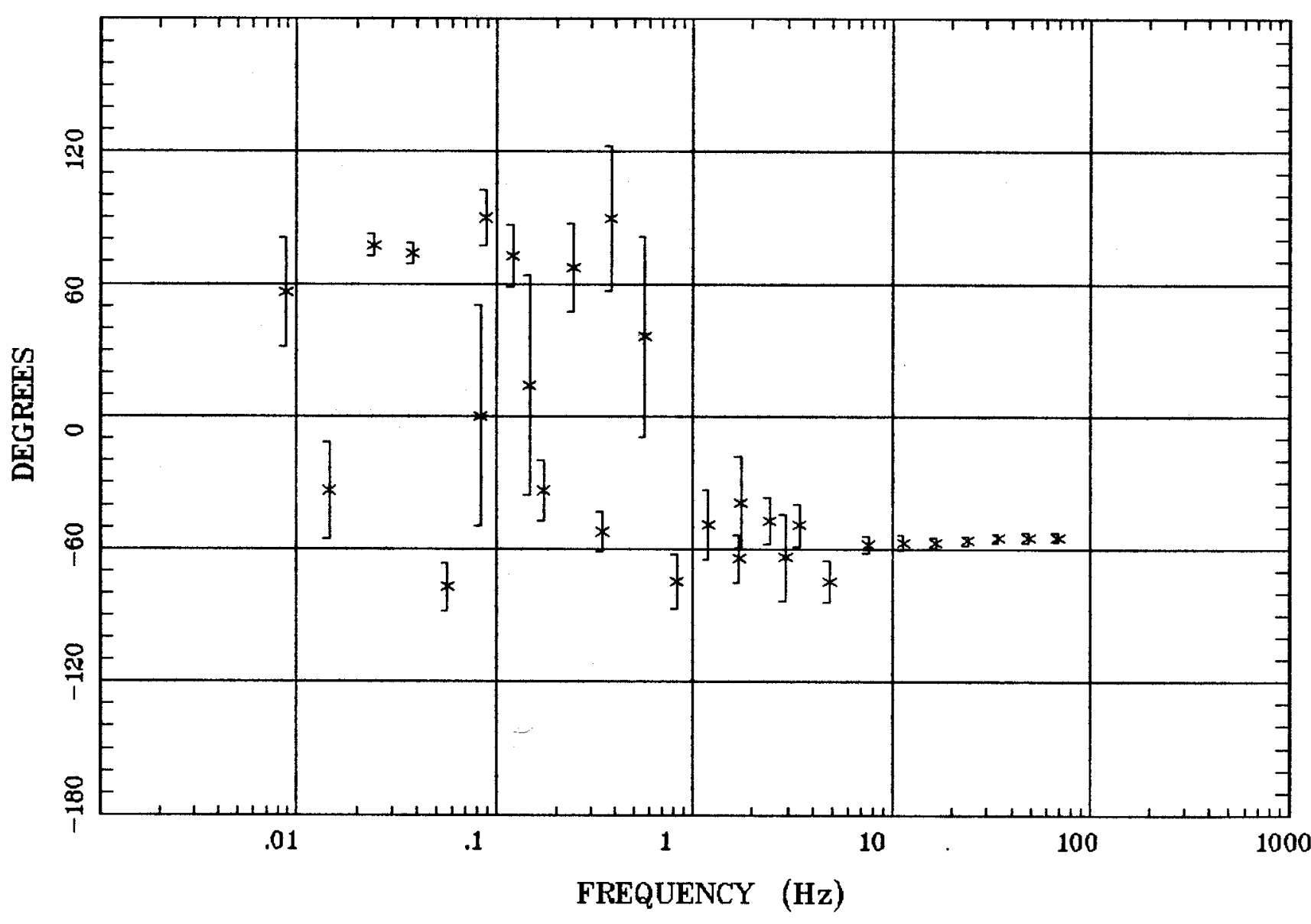

Client:

Remate: none

Acquired: 10:4 Aug 05, 1999 Survey Co:USGS
Rotation:

Filename: ar79.avg

Channels: Ch1 Ch2 Ch3 Ch4 Ch5 Ch3 Ch4

Platted: 10:56 Dec 08, 2000

< EMI - ElectroMagnetic Instruments 


\section{HzHx.x Coh HzHy.o}

Alligator Ridge

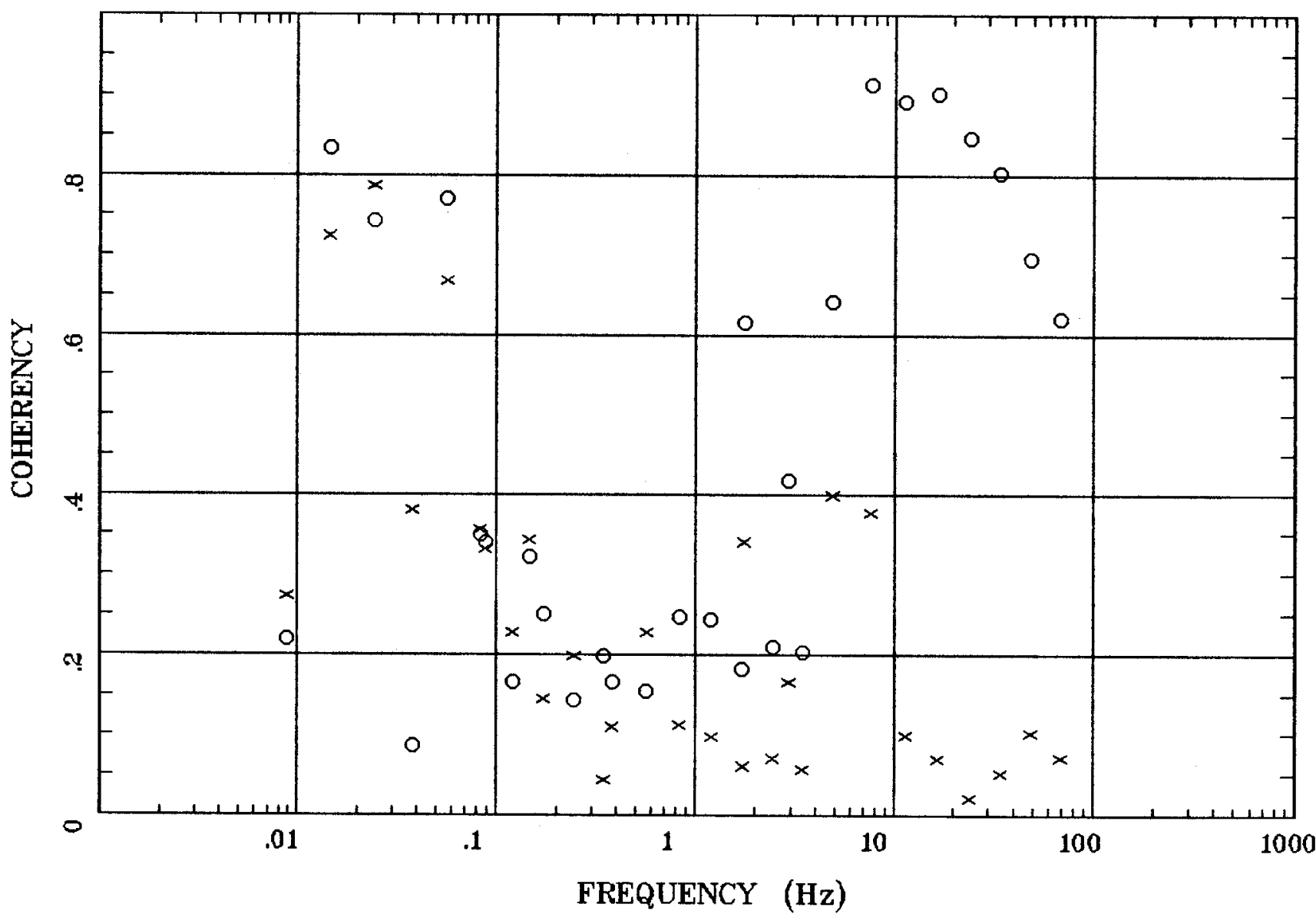

Client:

Remote: none

Acquired: 10:4 Aug 05, 1999 Survey Co:USGS
Rotation:

Filename: ar79.avg

Channels: Ch1 Ch2 Ch3 Ch4 Ch5 Ch3 Ch4 Plotted: 10:56 Dec 08, 2000

<EMI - ElectroMagnetic Instruments > 


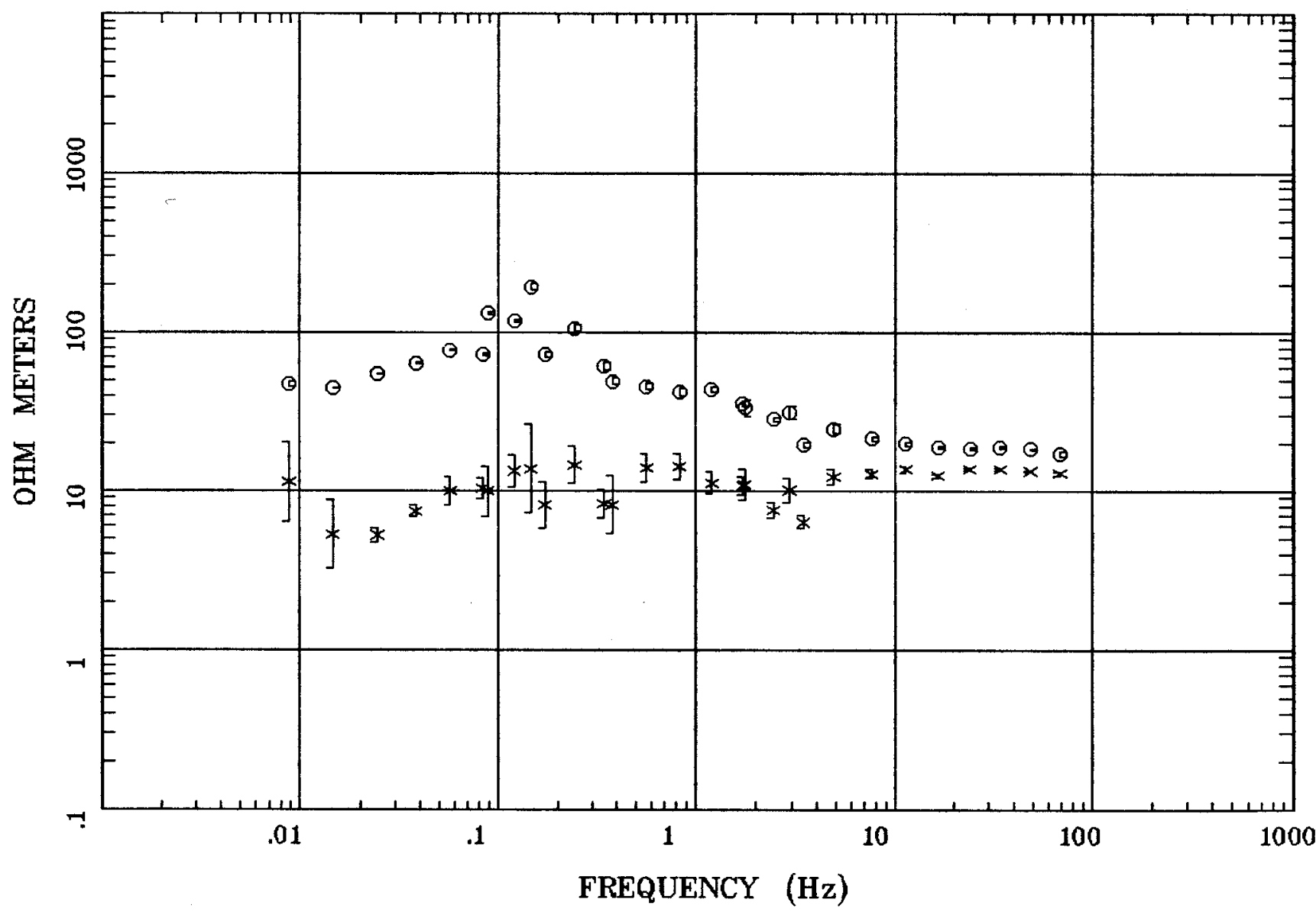

Client:

Remote: none

Acquired: 10:5 Aug 04, 1999

Survey co:USGS
Rotation:

Filename: ar77.avg

Channels: Ch1 Ch2 Ch3 Ch4 Ch5 Ch3 Ch4

Plotted: 10:54 Dec 08, 2000

< EMI - ElectroMagnetic Instruments > 


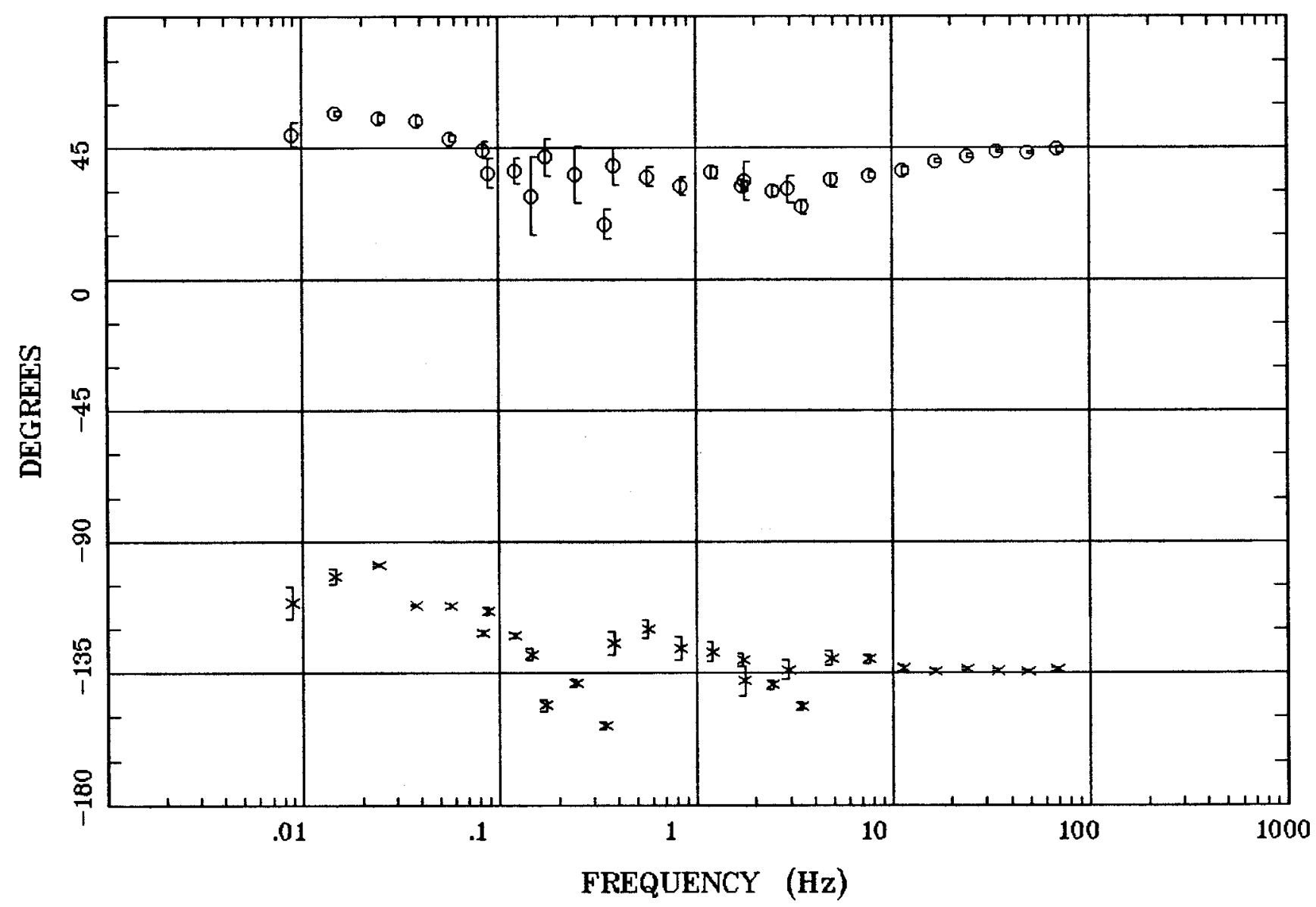

Client:

Remote: none

Acquired: 10:5 Aug 04, 1999 Survey Co:USGS
Rotation:

Filename: ar77.avg

Channels: Ch1 Ch2 Ch3 Ch4 Ch5 Ch3 Ch4

Plotted: 10:54 Dec 08, 2000

< EMI - ElectroMagnetic Instruments > 


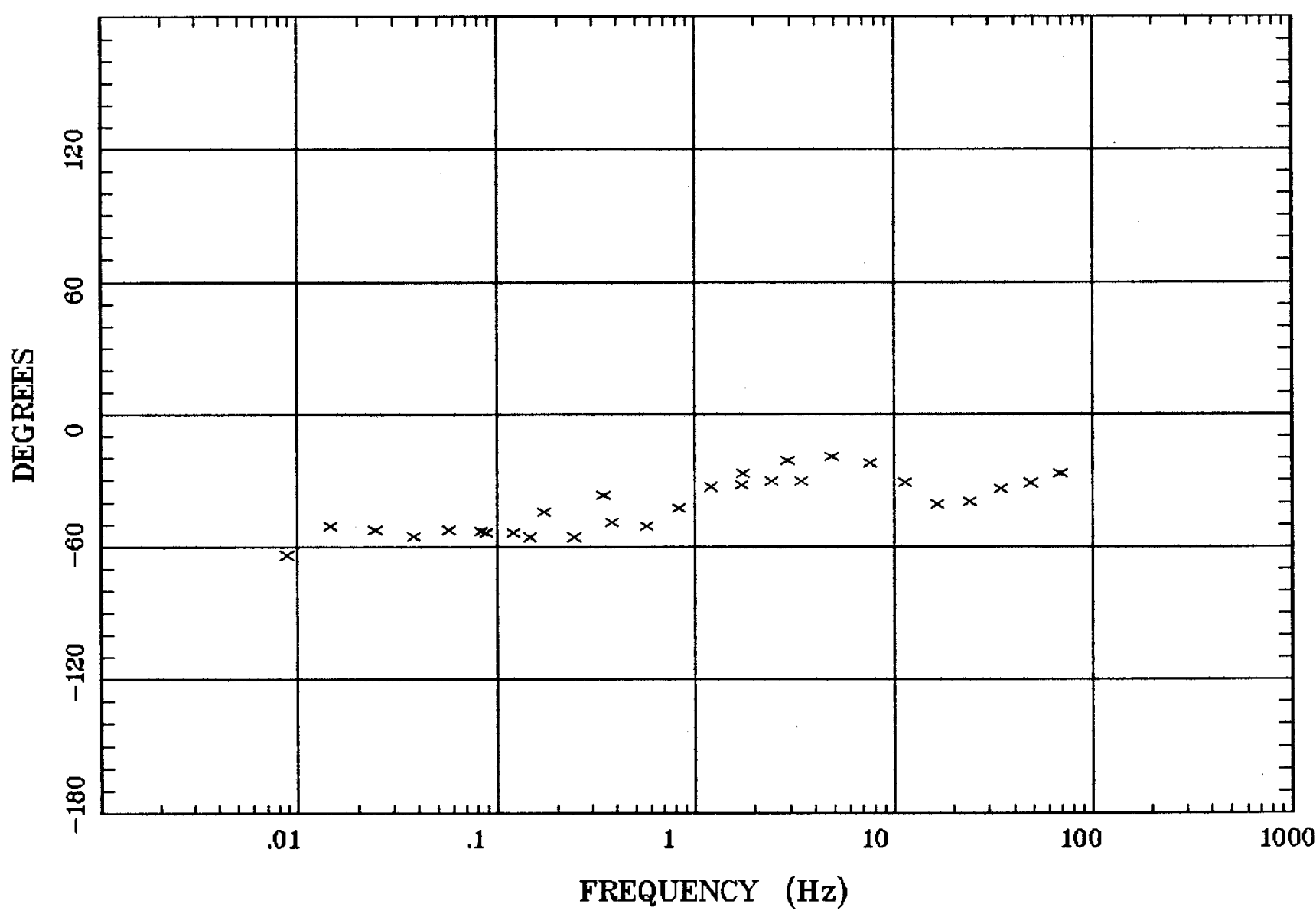

Client:

Remote: none

Acquired: 10:5 Aug 04, 1999

Survey Co:USGS
Rotation:

Filename: ar77.avg

Channels: Ch1 Ch2 Ch3 ch4 Ch5 Ch3 Ch4 Plotted: 10:54 Dec 08, 2000

$<$ EMI - ElectroMagnetic Instruments 


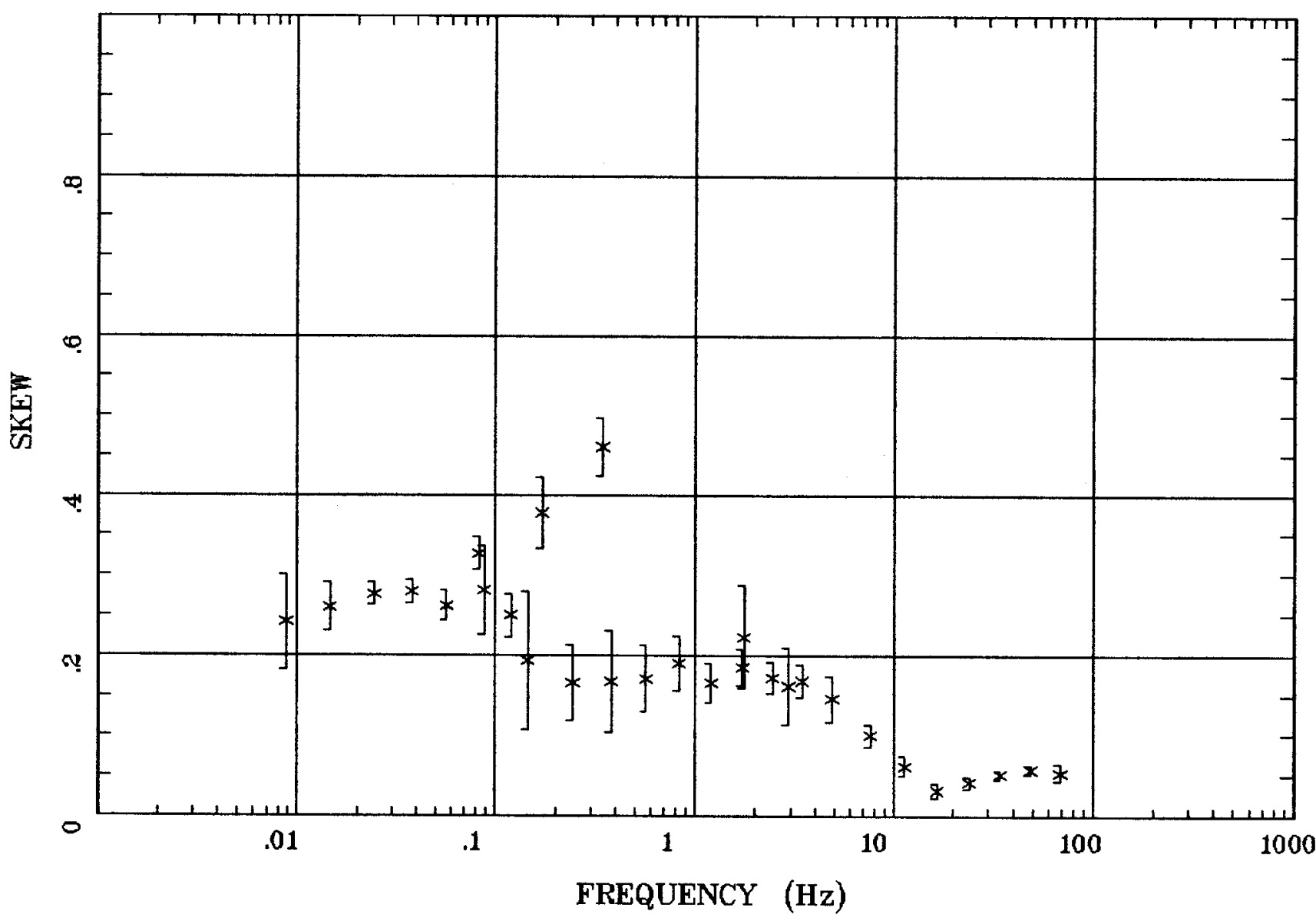

Client:

Remote: none

Acquired: 10:5 Aug 04, 1999

Survey Co:USGS
Rotation:

Filename: ar77.avg

Channels: Ch1 Ch2 Ch3 Ch4 Ch5 Ch3 Ch4

Plotted: 10:54 Dec 08, 2000

< EMI - ElectroMagnetic Instruments > 


\section{E MULT Coh.}

Long Valley

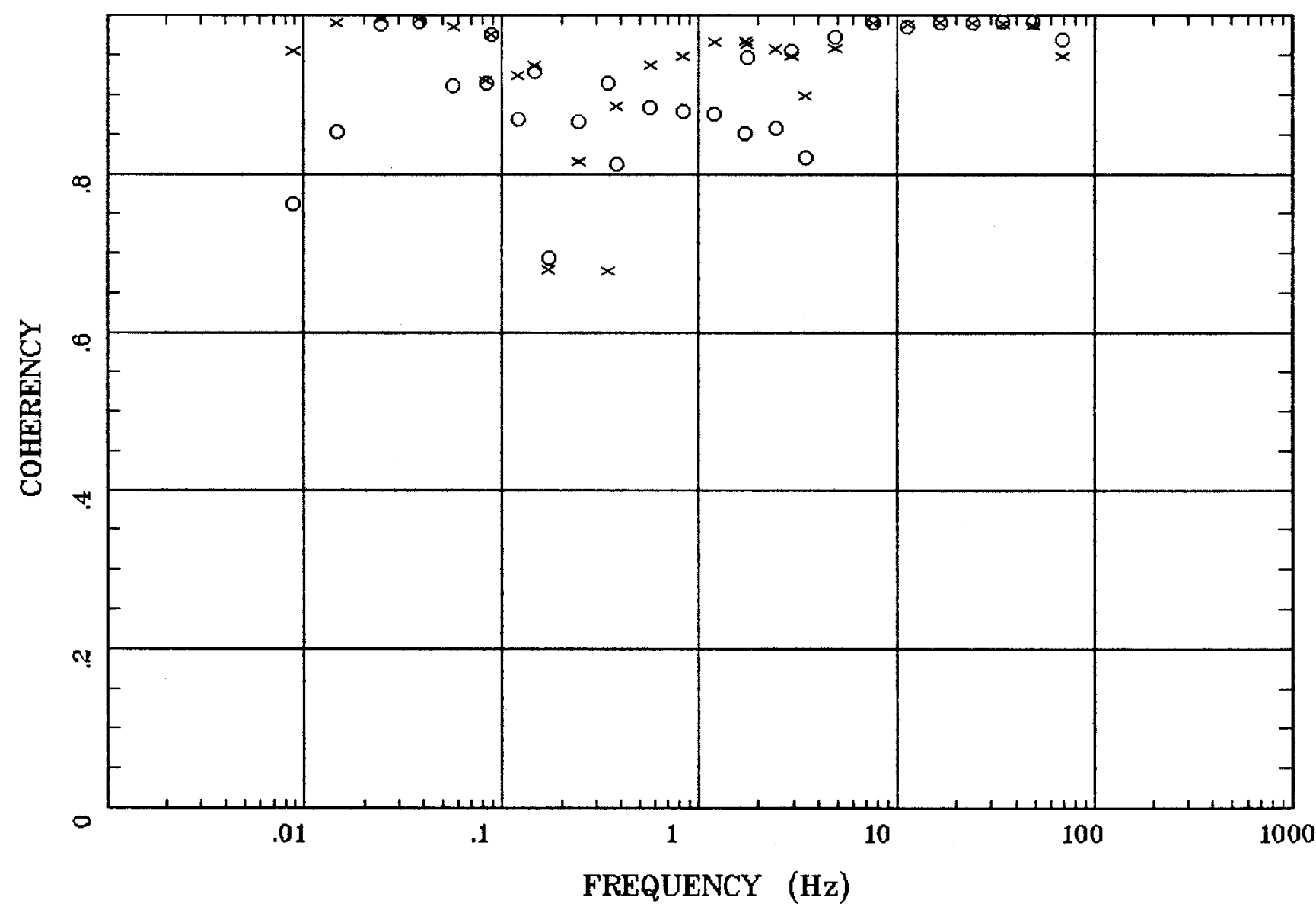

Client:

Remote: none

Acquired: 10:5 Aug 04, 1999

Survey Co:USGS
Rotation:

Filename: ar77.avg

Channels: Ch1 Ch2 Ch3 ch4 Ch5 Ch3 Ch4 Plotted: 10:54 Dec 08, 2000

< EMI - ElectroMagnetic Instruments 
POLAR PLOTS

\section{Long Valley}

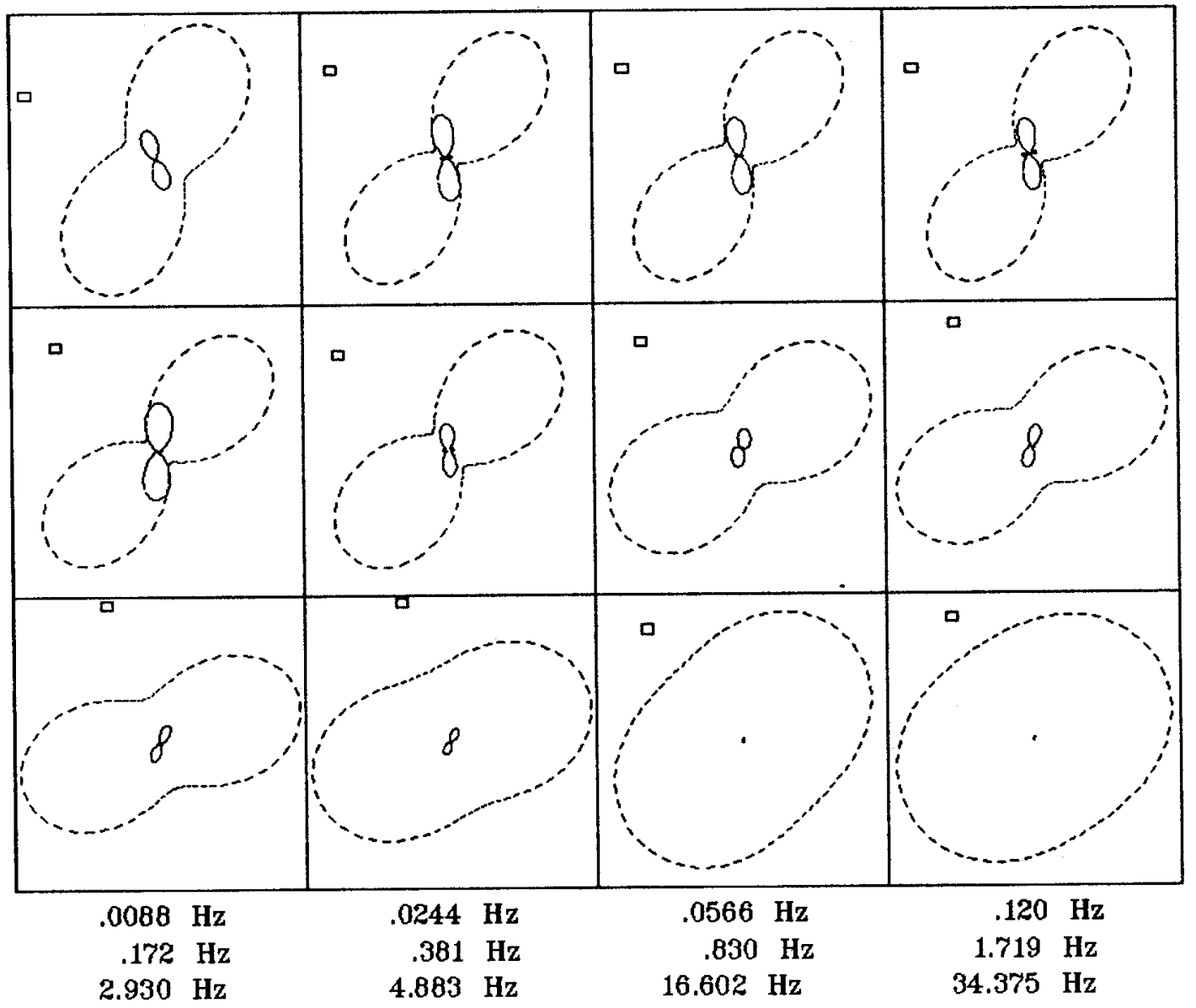

Rotation:

Client:

Remote: none

Acquired: 10:5 Aug 04, 1999 Survey Co:USGS
Filename: ar77.avg

Channels: Ch1 Ch2 Ch3 Ch4 Ch5 Ch3 Ch4

Plotted: 10:54 Dec 08, 2000

<EMI - ElectroMagnetic Instruments > 


\section{TIPPER MAGNITUDE}

\section{Long Valley}

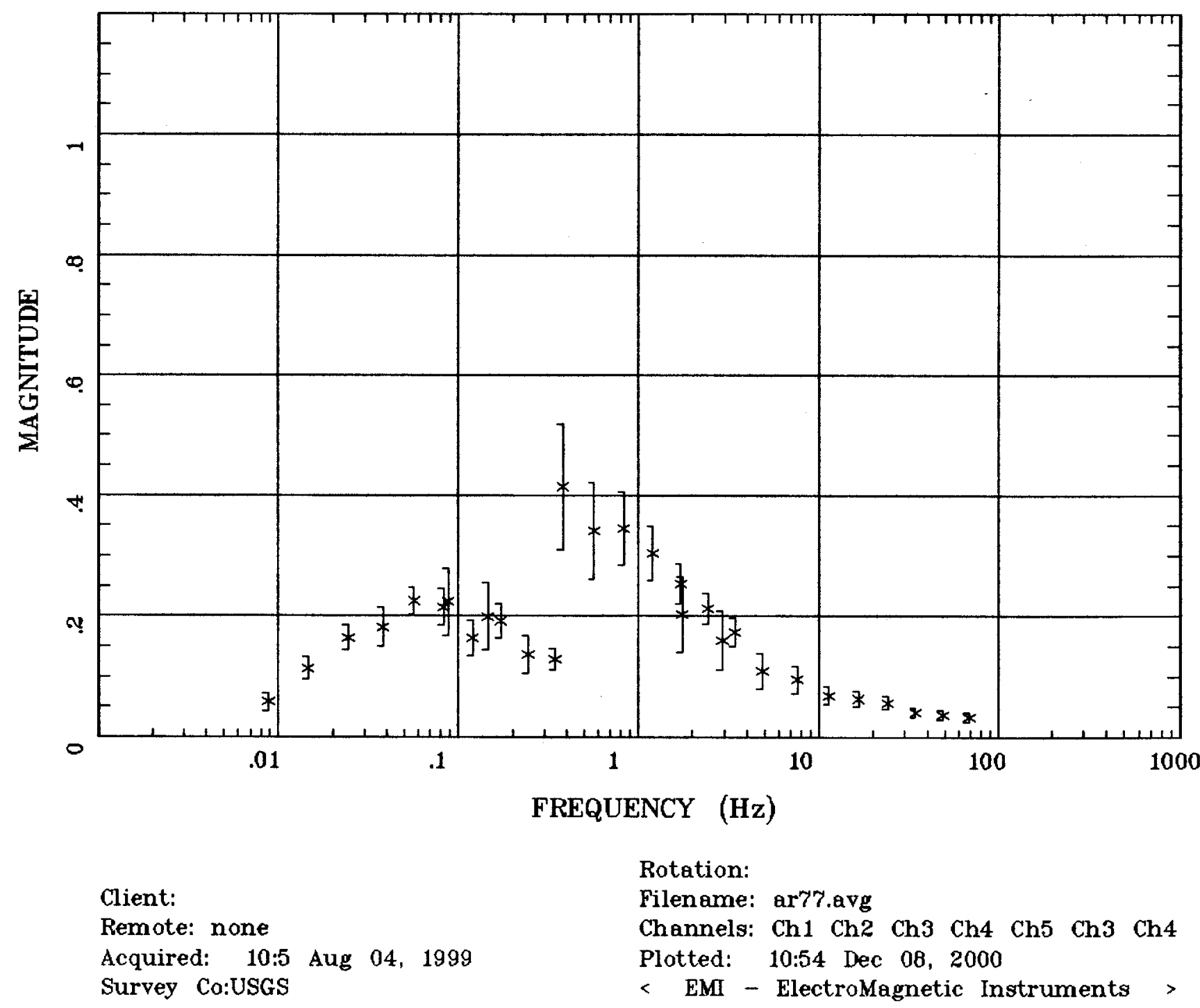




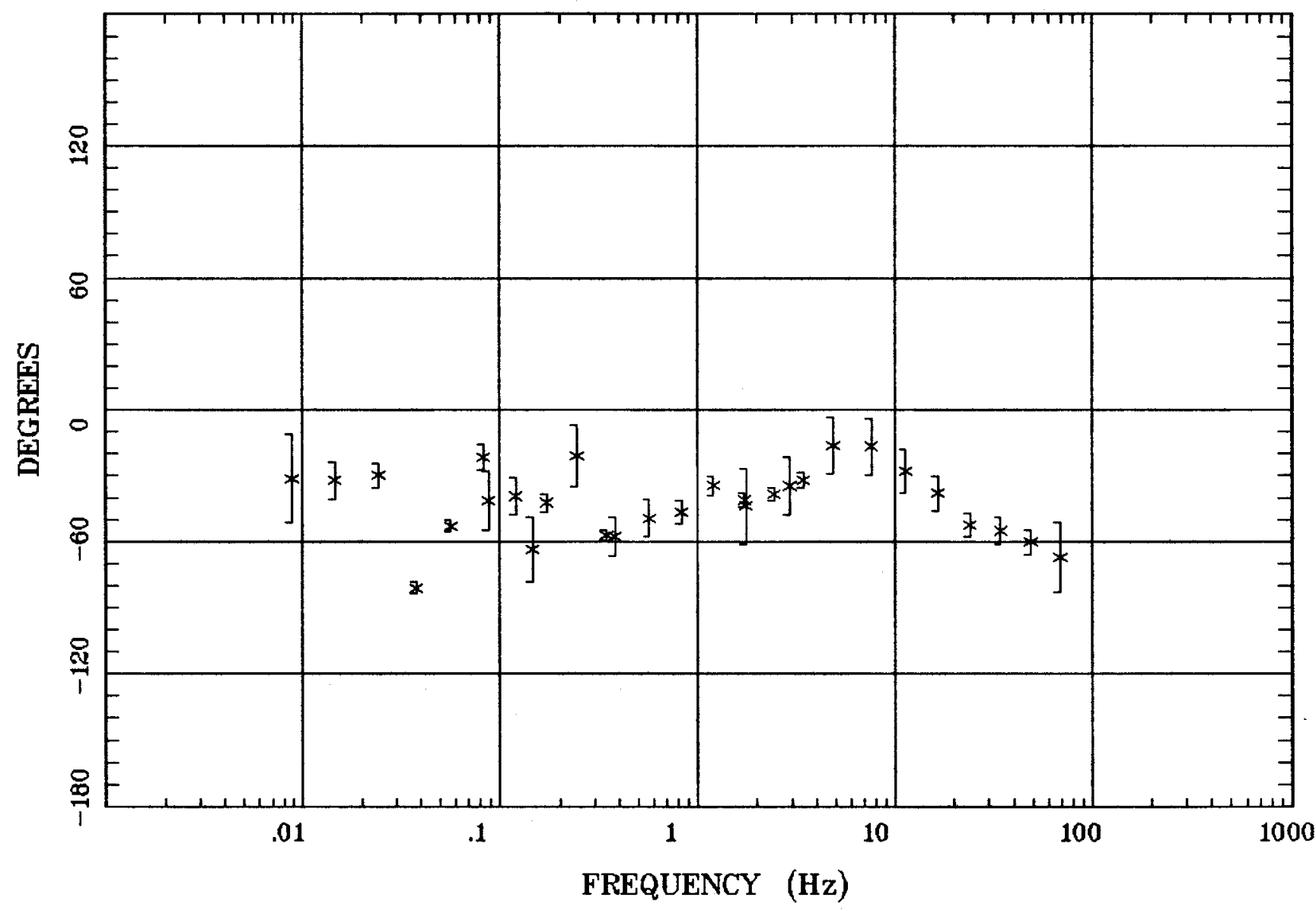

Client:

Remote: none

Acquired: 10:5 Aug 04, 1999 Survey Co:USGS
Rotation:

Filename: ar77.avg

Channels: Ch1 Ch2 Ch3 ch4 Ch5 Ch3 Ch4 Plotted: 10:54 Dec 08, 2000

< EMI - ElectroMagnetic Instruments 


\section{HzHx.x Coh HzHy.o Long Valley}

Station 77

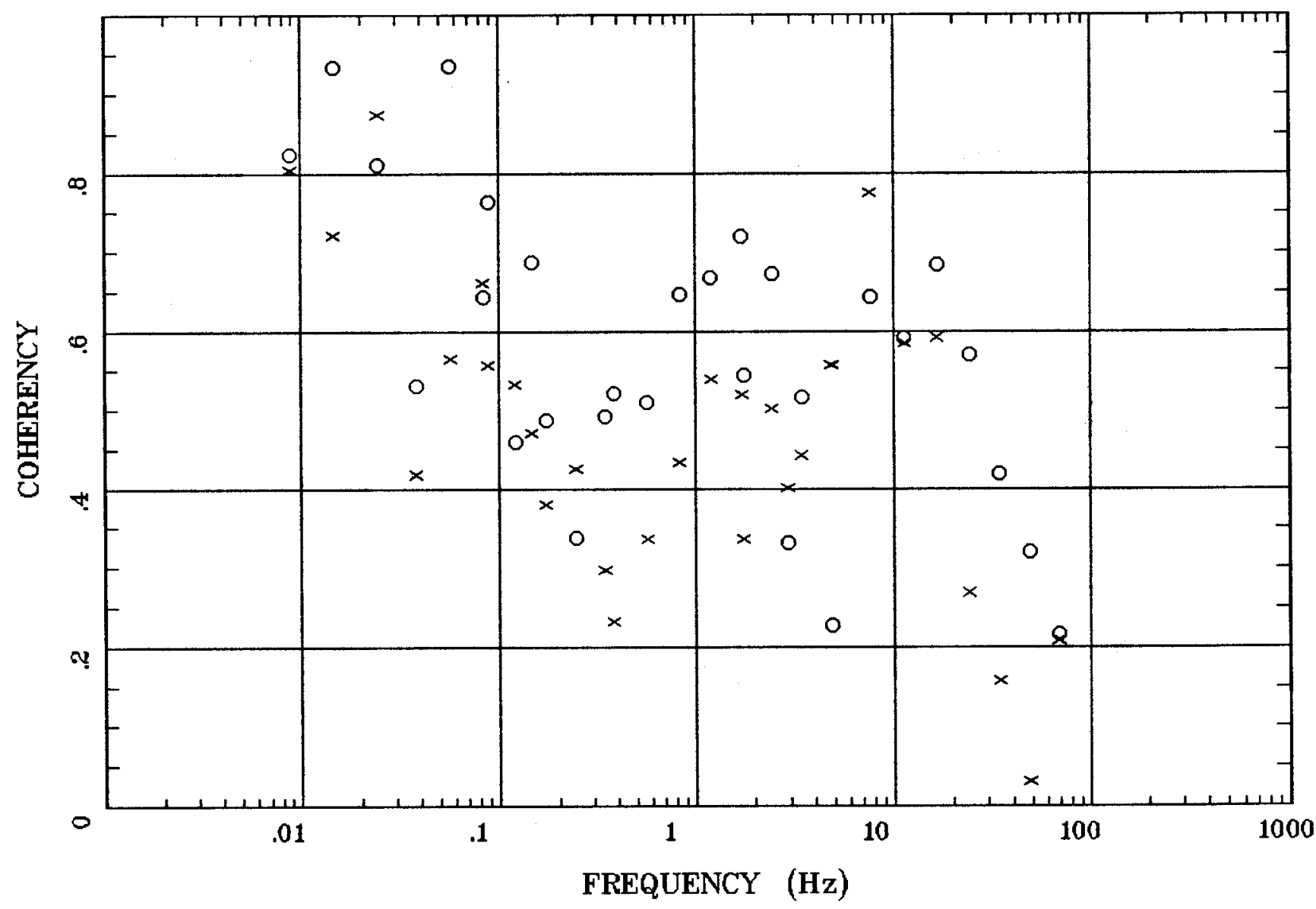

Client:

Remote: none

Acquired: 10:5 Aug 04, 1999

Survey Co:USGS
Rotation:

Filename: ar77.avg

Channels: Ch1 Ch2 Ch3 Ch4 Ch5 Ch3 Ch4

Plotted: 10:54 Dec 08, 2000

$<$ EMI - ElectroMagnetic Instruments > 


\section{Station 78}

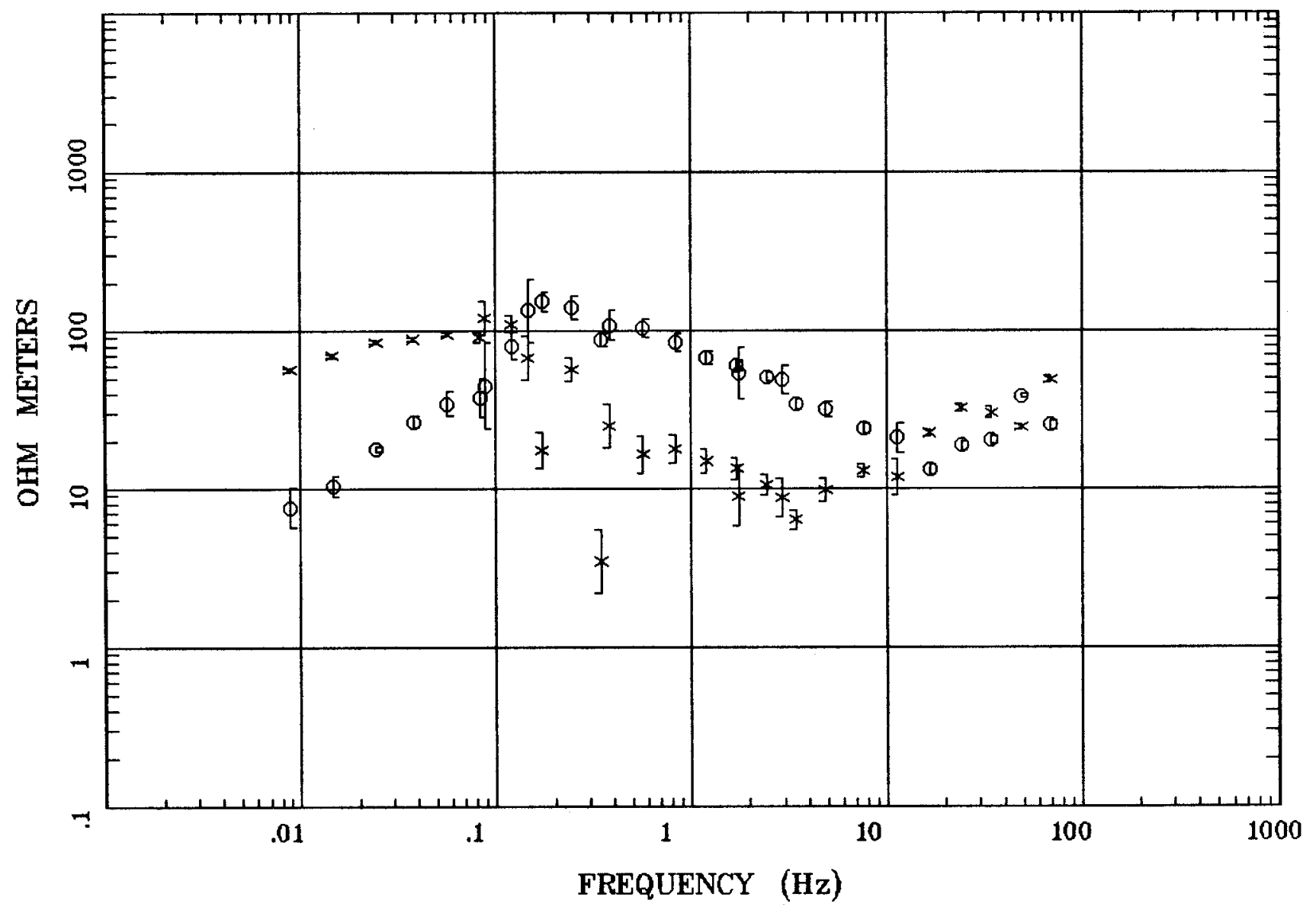

Client:

Remote: none

Acquired: 13:4 Aug 04, 1999 Survey Co:USGS
Rotation:

Filename: ar78.avg

Channels: Ch1 Ch2 Ch3 Ch4 Ch5 Ch3 Ch4 Plotted: 10:55 Dec 08, 2000

< EMI - ElectroMagnetic Instruments 
IMPEDANCE PHASE

\section{Long Valley}

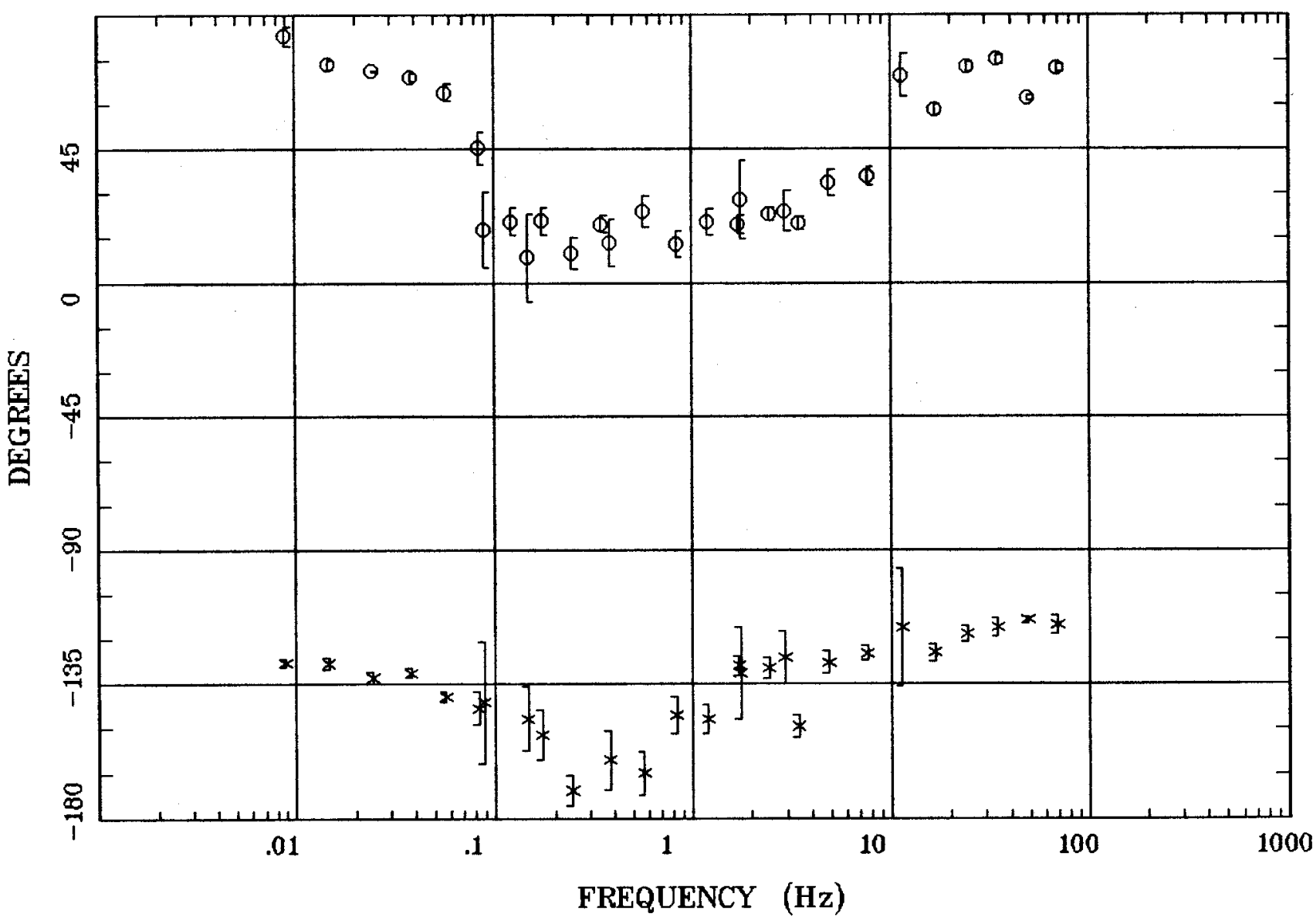

Client:

Remote: none

Acquired: 13:4 Aug 04, 1999 Survey Co:USGS
Rotation:

Filename: ar78.avg

Channels: Ch1 Ch2 Ch3 Ch4 Ch5 Ch3 Ch4 Plotted: 10:55 Dec 08, 2000

< EMI - ElectroMagnetic Instruments > 


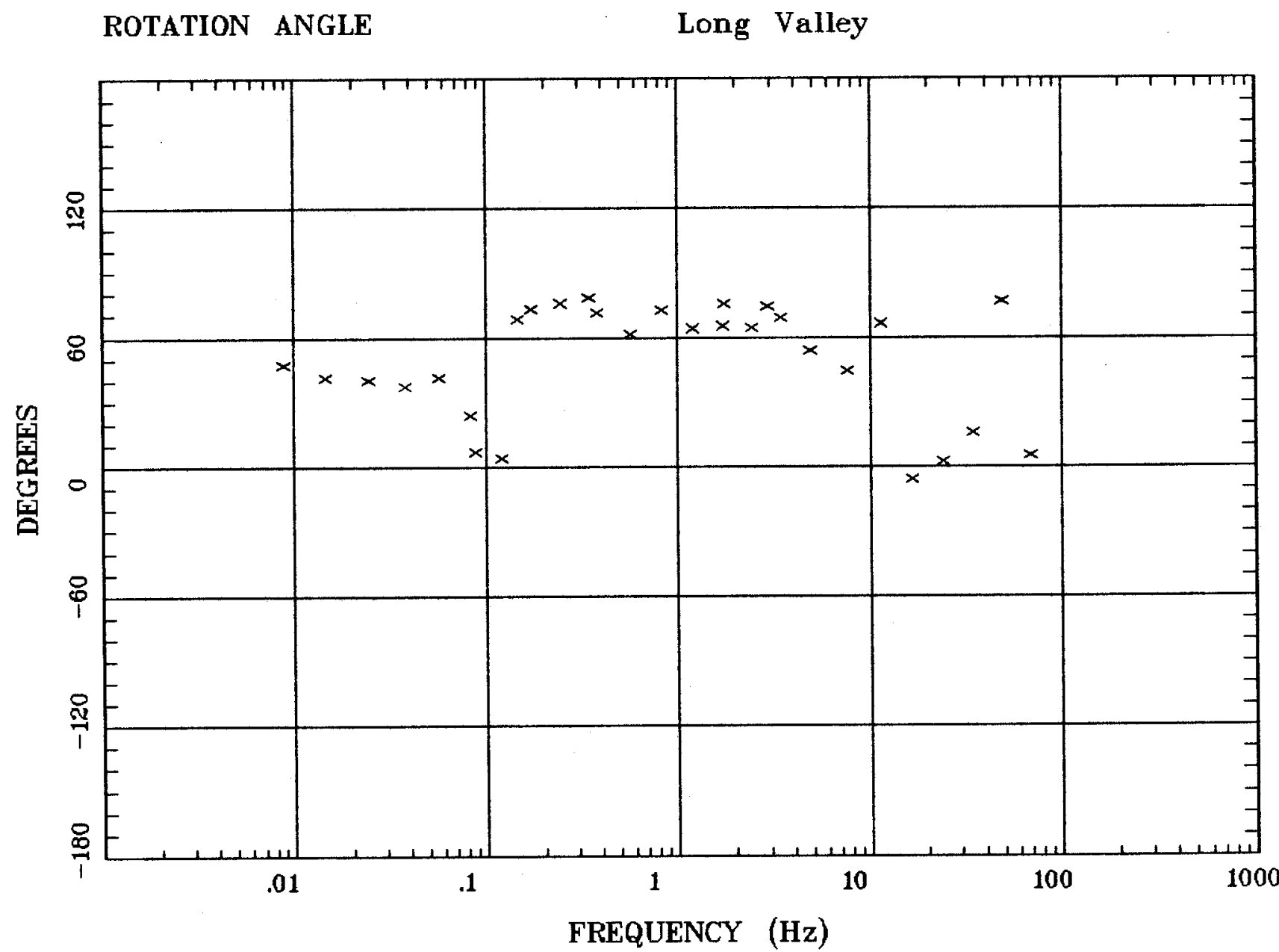

Client:

Remote: none

Acquired: 13:4 Aug 04, 1999

Survey Co:USGS

Rotation:

Filename: ar78.avg

Channels: Ch1 Ch2 Ch3 Ch4 Ch5 Ch3 Ch4

Plotted: 10:55 Dec 08, 2000

< EMI - ElectroMagnetic Instruments > 


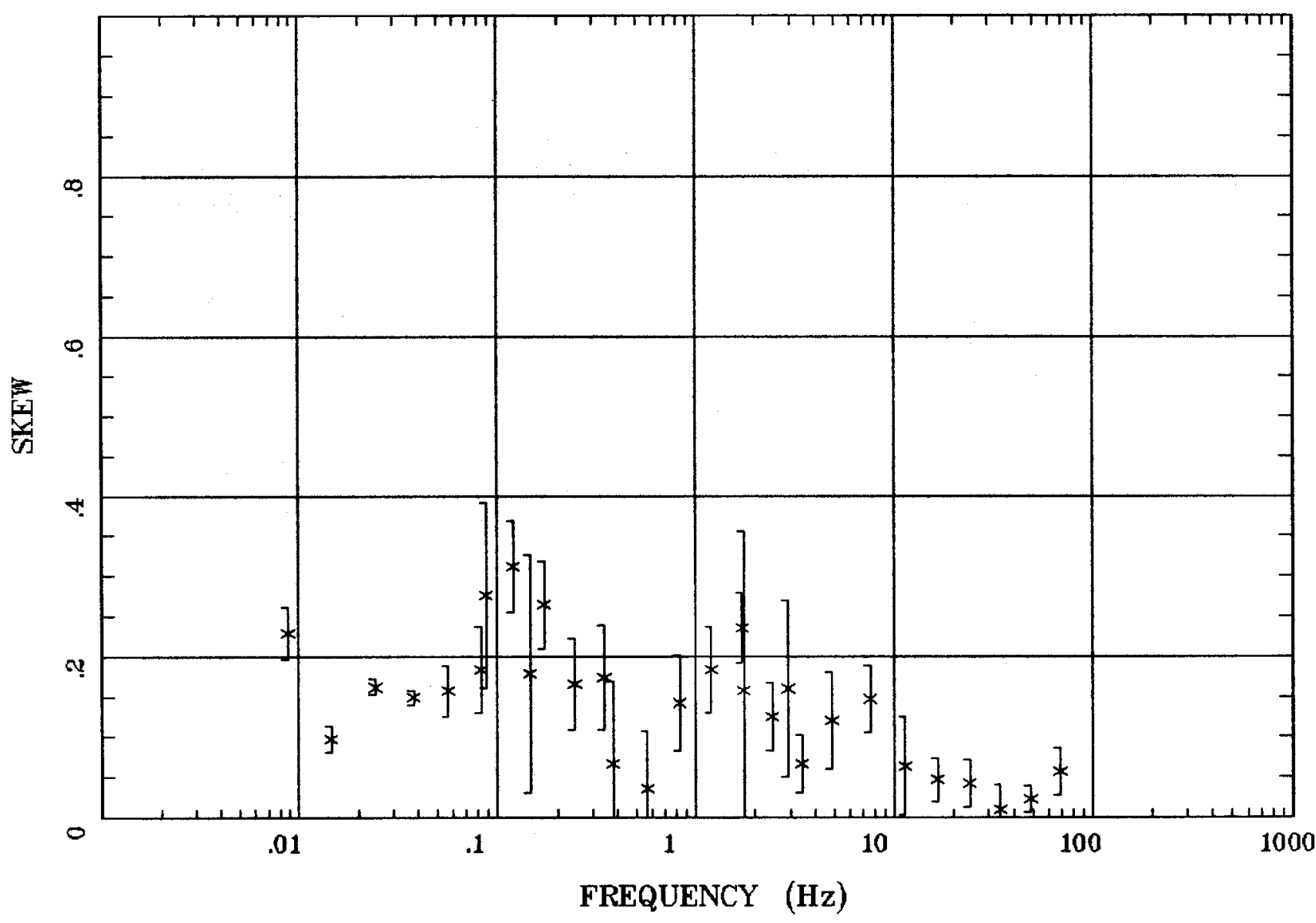

Client:

Remote: none

Acquired: 13:4 Aug 04, 1999 Survey Co:USGS
Rotation:

Filename: ar78.avg

Channels: Ch1 Ch2 Ch3 ch4 ch5 Ch3 Ch4 Plotted: 10:55 Dec 08, 2000

$<$ EMI - ElectroMagnetic Instruments 


\section{E MULT Coh.}

\section{Long Valley}

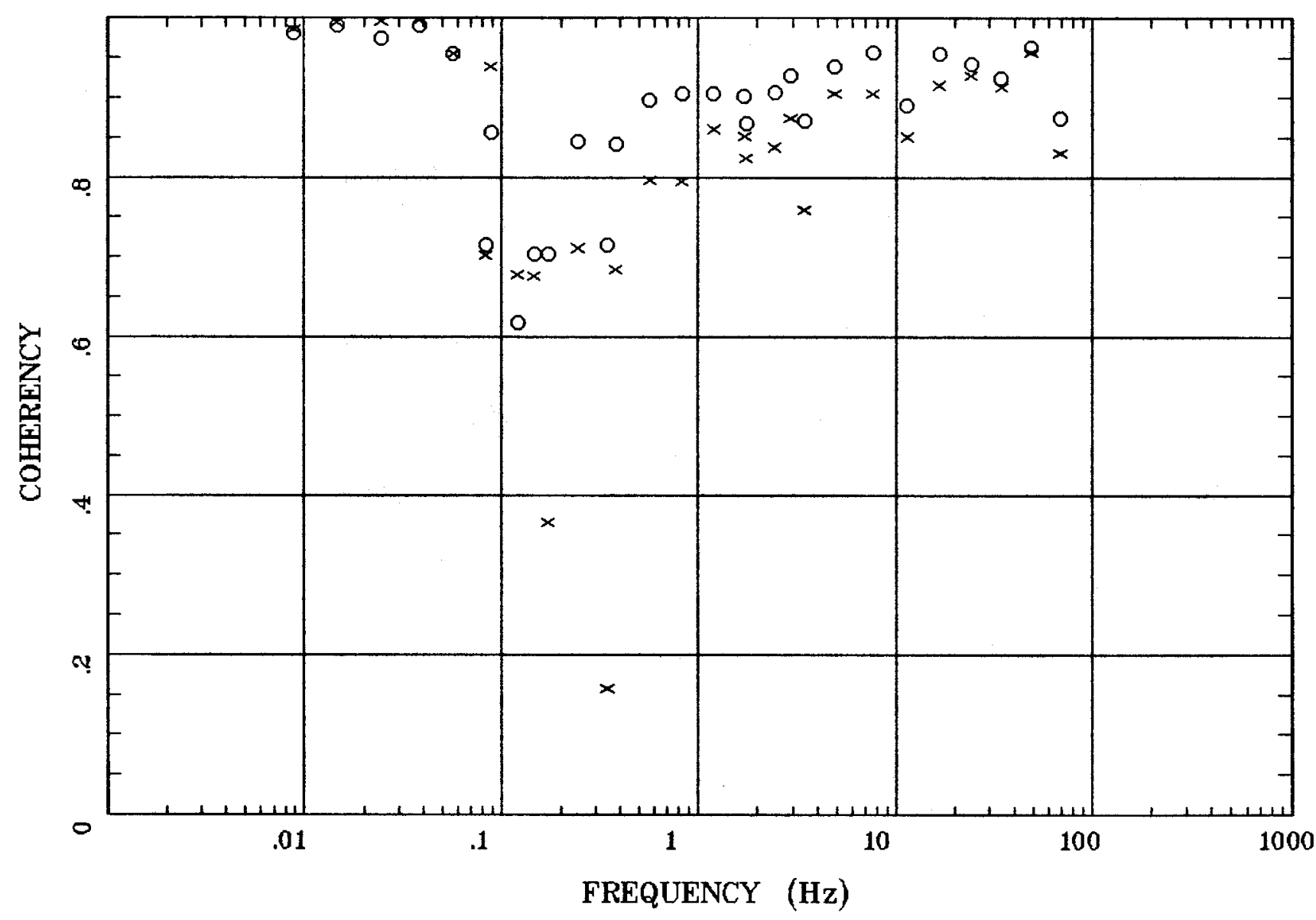

Client:

Remote: none

Acquired: 13:4 Aug 04, 1999

Survey Co:USGS
Rotation:

Filename: ar78.avg

Channels: Ch1 Ch2 Ch3 Ch4 Ch5 Ch3 Ch4 Plotted: 10:55 Dec 08, 2000

< EMI - ElectroMagnetic Instruments 


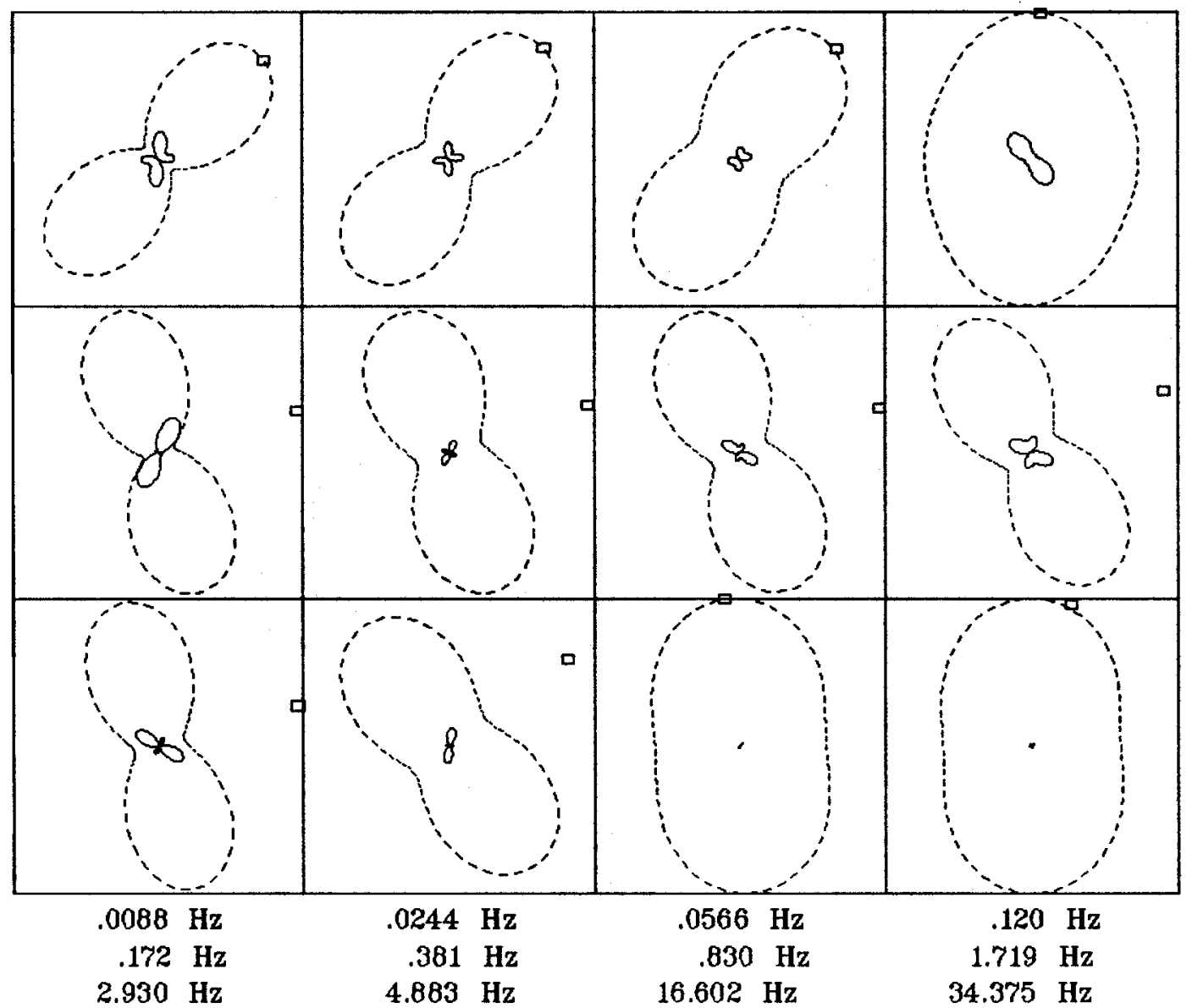

Client:

Remote: none

Acquired: 13:4 Aug 04, 1999

Survey Co:USGS
Rotation:

Filename: ar78.avg

Channels: Ch1 Ch2 Ch3 Ch4 Ch5 Ch3 Ch4 Plotted: 10:55 Dec 08, 2000

$<$ EMI - ElectroMagnetic Instruments 